\title{
CORRECTIVE ACTION DECISION DOCUMENT/CLOSURE REPORT FOR CORRECTIVE ACTION UNIT 476: AREA 12 T-TUNNEL MUCKPILE, NEVADA TEST SITE
}

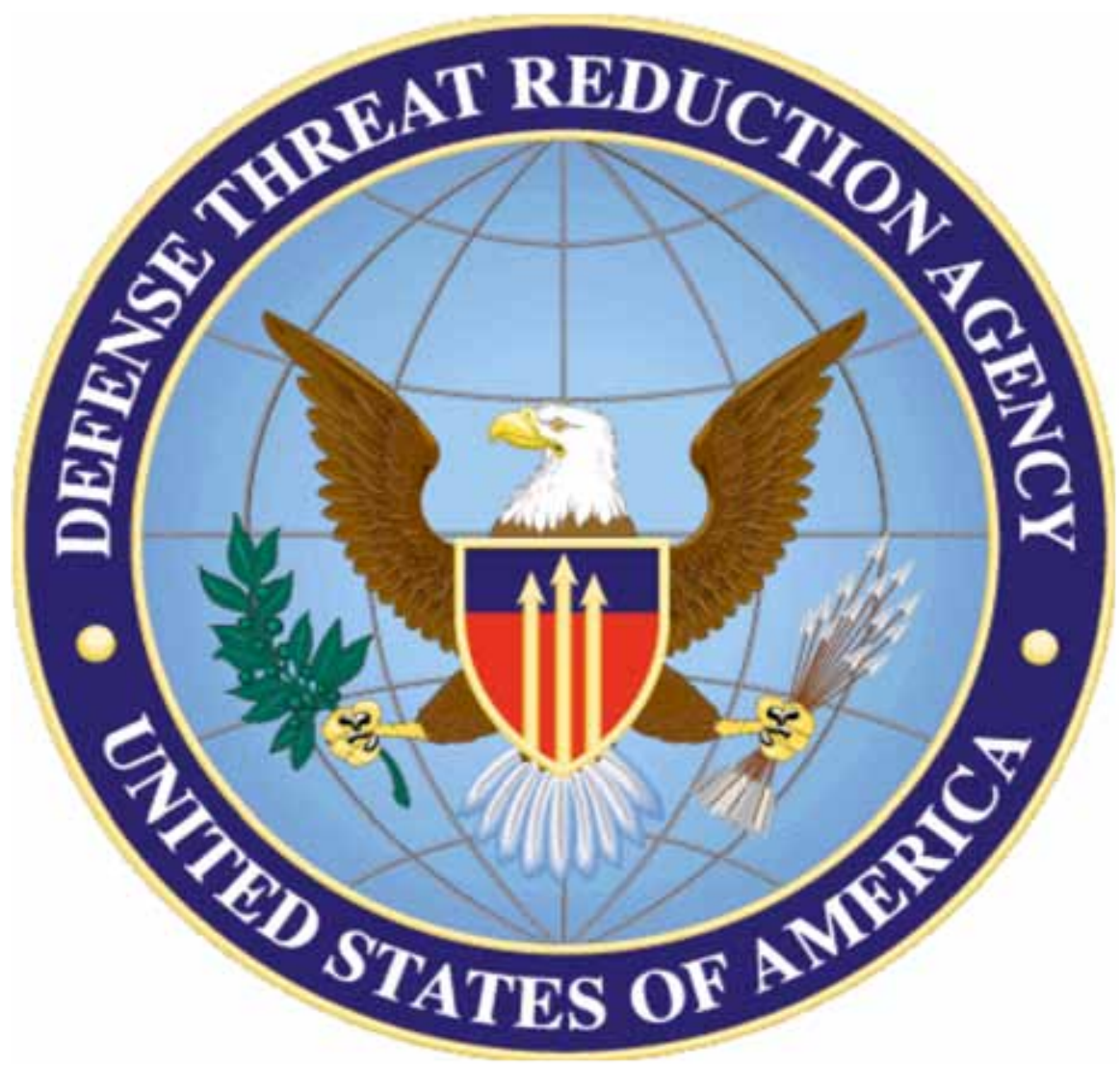

Controlled Copy No:

Revision No.: 0

May 2007

(Republished March 2010) 


\title{
DISCLAIMER
}

Reference herein to any specific commercial product, process, or service by trade name, trademark, manufacturer, or otherwise, does not necessarily constitute or imply its endorsement, recommendation, or favoring by the United States Government or any agency thereof.

This report has been reproduced directly from the best available copy.

Available for sale to the public from:

\author{
U.S. Department of Commerce \\ National Technical Information Service \\ 5301 Shawnee Road \\ Alexandria, VA 22312 \\ Telephone: (800) 553-6847 \\ Fax: (703) 605-6900 \\ E-mail: orders@ntis.gov \\ Online ordering: http://www.ntis.gov/help/ordermethods.aspx
}

Available electronically at http://www.osti.gov/bridge.

Available for a processing fee to the U.S. Department of Energy and its contractors, in paper, from:
U.S. Department of Energy
Office of Scientific and Technical Information
P.O. Box 62
Oak Ridge, TN 37831-0062
Telephone: (865) 576-8401
Fax: (865) 576-5728
E-mail: reports@adonis.osti.gov

(This document was republished. A front cover and back cover were added to reflect document numbers, disclaimer, availability, and library distribution. No other changes were made.) 


\title{
CORRECTIVE ACTION DECISION DOCUMENT/CLOSURE REPORT FOR CORRECTIVE ACTION UNIT 476: AREA 12 T-TUNNEL MUCKPILE, NEVADA TEST SITE
}

\author{
Prepared by \\ Defense Threat Reduction Agency \\ Mercury, Nevada
}

Controlled Copy No.

Revision No.: 0

May 2007

(Republished March 2010) 


\title{
CORRECTIVE ACTION DECISION DOCUMENT/CLOSURE REPORT FOR CORRECTIVE ACTION UNIT 476: \\ AREA 12 E-TUNNEL MUCKPILE, NEVADA TEST SITE
}

\author{
Approved by: $\frac{\text { /s/David C. Loewer for Tiffany A. Lantow }}{\text { Tiffany A. Lantow }} \quad$ Date: $\underline{3 / 26 / 2007}$ \\ Environmental Program Manager \\ Nevada Operations Office \\ Defense Threat Reduction Agency
}




\section{Table of Contents}

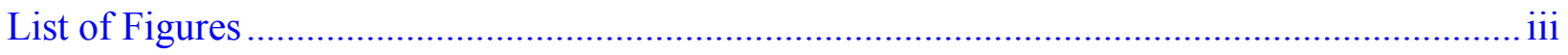

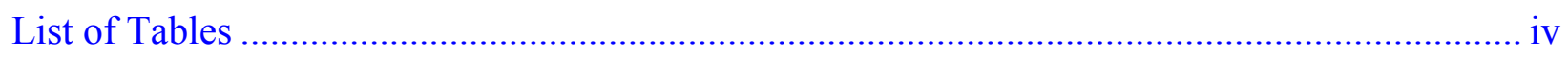

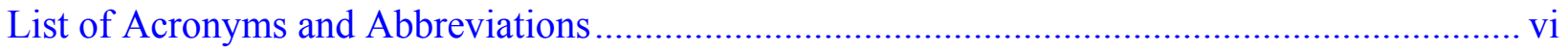

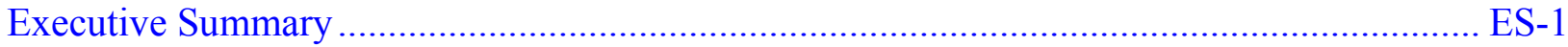

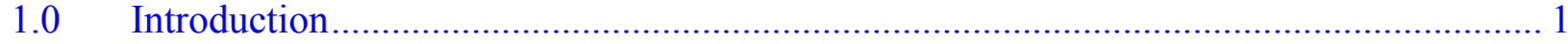

1.1 Purpose

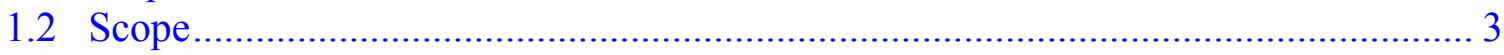

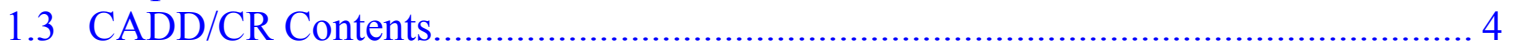

2.0 Corrective Action Investigation Summary ........................................................ 5

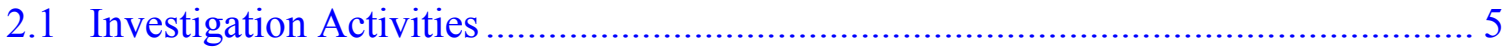

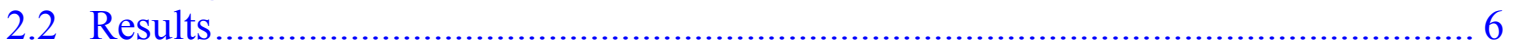

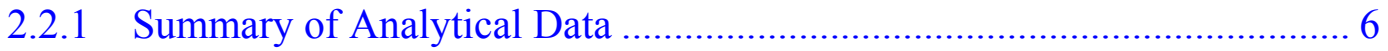

2.2.1.1 Muckpile (CAS 12-06-02) .................................................... 8

2.2.1.2 Native Material Under the Muckpile .................................... 10

2.2.1.3 Shallow Soils on the Muckpile .............................................. 11

2.2.1.4 T-Tunnel Background ....................................................... 13

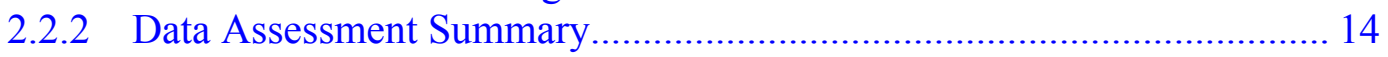

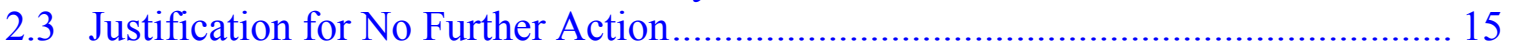

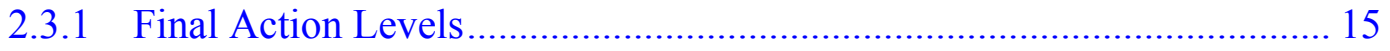

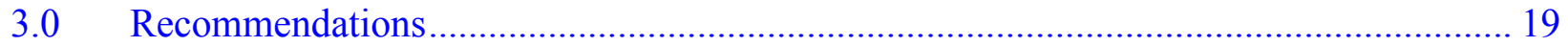

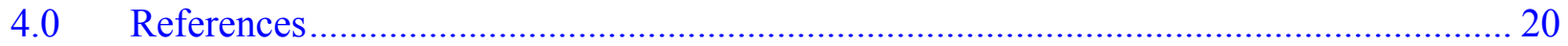

Appendix A - Corrective Action Investigation Report for CAU 476, Area 12 T-Tunnel Muckpile, Nevada Test Site

\section{Appendix B - Data Quality Objective Process and Methodology}

B.1.0 Data Quality Objective Process and Methodology .............................................. B-1

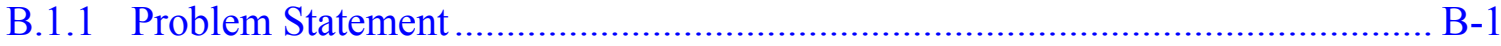

B.1.2 Definition of the Decision.......................................................................... B-1

B.1.3 Identification of Inputs to the Decision ................................................. B-2

B.1.4 Definition of Study Boundaries ............................................................. B-2

B.1.5 Determination of Decision Rules.............................................................. B-2

B.1.6 Specification on Decision Error Limits ….................................................... B-3

B.1.7 Optimization of the Design for Obtaining Data......................................... B-3

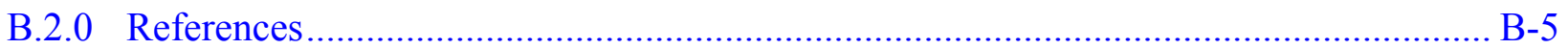




\section{Table of Contents (Continued)}

\section{Appendix C - Data Assessment}

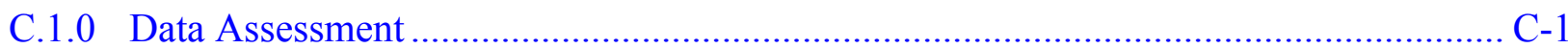

C.1.1 Review the DQOs and Sampling Design......................................................

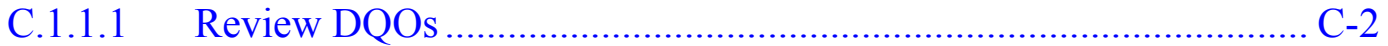

C.1.1.2 Sampling Design ..................................................................... C-11

C.1.2 Conduct a Preliminary Data Review.......................................................... C-11

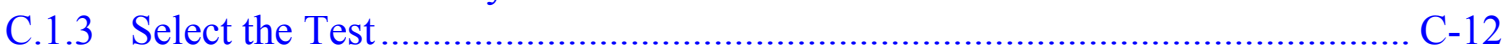

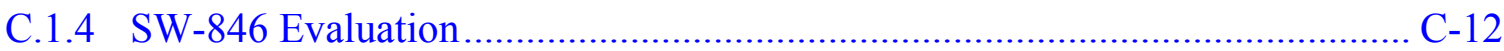

C.1.5 Verify the Assumptions ............................................................................ C-14

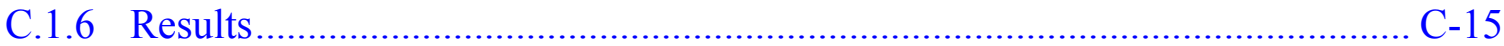

C.1.6.1 Decision Rules for CAU 476 ........................................................ C-15

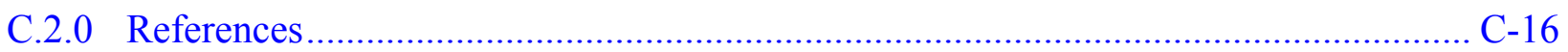

\section{Appendix D - Risk Assessment for CAU 476}

D.1.0 Risk-Based Corrective Action Process ...................................................................... D-1

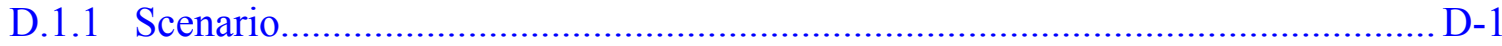

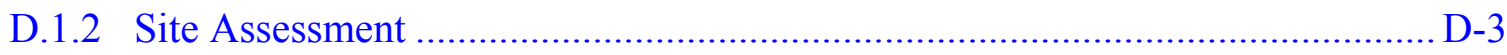

D.1.3 Site Classification and Initial Response Action............................................... D-4

D.1.4 Development of Tier 1 Lookup Table of Risk-Based

Screening Level Selection.............................................................................. D-5

D.1.5 Exposure Pathway Evaluation ………………………………………….... D-6

D.1.6 Comparison of Site Conditions with Tier 1 Risk-Based Screening Levels ........ D-6

D.1.7 Evaluation of Tier 1 Results ....................................................................... D-7

D.1.8 Tier 1 Remedial Action Evaluation ............................................................... D-7

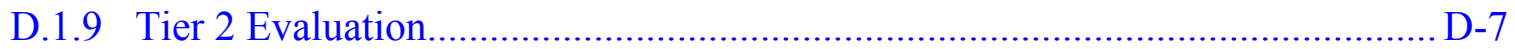

D.1.10 Development of Tier 2 Table of SSTLs........................................................ D-7

D.1.11 Comparison of Site Conditions with Tier 2 FALs ............................................ D-9

D.1.12 Tier 2 Remedial Action Evaluation .......................................................... D-10

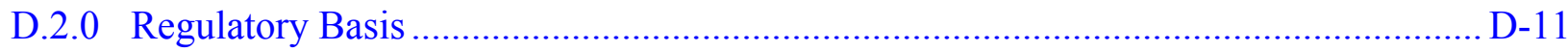

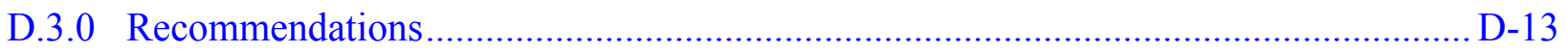

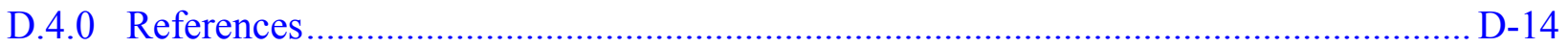

Attachment A - Derivation of Residual Radioactive Material Guidelines for Radionuclides in Soil at Corrective Action Unit (CAU) 476, Area 12 T-Tunnel Muckpile, Nevada Test Site, Nevada Exhibit 1 - RESRAD Summary Report: CAU 476

\section{Appendix E - Closure Summary}

E.1.0 Closure Summary 


\section{List of Figures}

Number

Title

Page

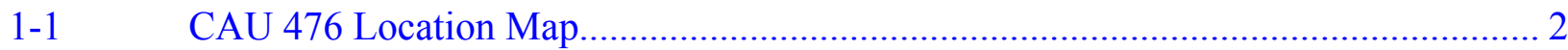

2-1 T-Tunnel Muckpile Sample Locations ………………………............................. 7

D.1-1 ASTM Method E 1739-95 Risk-Based Corrective Action Decision Process........... D-2

E.1-1 CAU 476 Area 12 T-Tunnel Sites ........................................................................ 


\section{List of Tables}

Number

Title

Page

2-1 Maximum Reported Chemical Values for Muck in

CAS 12-06-02, Muckpile 8

2-2 Maximum Reported Radiological Values for Muck in

CAS 12-06-02, Muckpile 9

2-3 Maximum Reported Chemical Values for Native Material Under

CAS 12-06-02 Muckpile

2-4 Maximum Reported Radiological Values for Native Material

Under CAS 12-06-02 Muckpile.

2-5 Maximum Reported Chemical Values for Shallow Soils at

CAS 12-06-02 Muckpile.

2-6 Maximum Reported Radiological Values for Shallow Soils at

CAS 12-06-02 Muckpile.

2-7 Maximum Reported Chemical Values for T-Tunnel Background .............................. 13

2-8 Maximum Reported Radiological Values for T-Tunnel Background ........................ 14

2-9 Tier 2 FALs and CAU 476 Results for Hazardous Constituents of Diesel ................. 17

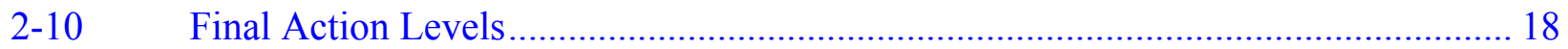

C.1-1 CAU 476 Number of Soil Samples Submitted per Analyte ...................................... C-3

C.1-2 Chemical Analytes Failing Sensitivity Criteria for CAU 476 …............................. C-4

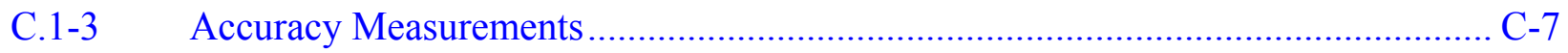

C.1-4 SW-846 Evaluation of the Number of Samples and

Comparison of $90 \%$ Confidence Level with the PAL ............................................ C-13

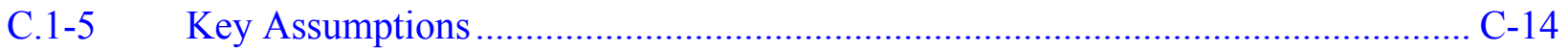

D.1-1 Maximum Reported Chemical Values for Tier 1 Comparison ................................. D-3

D.1-2 Maximum Reported Radiological Values for Tier 1 Comparison............................. D-4

D.1-3 COPCs Detected Above Preliminary Action Levels …………………….............. D-6 


\section{List of Tables (Continued)}

Number

Title

Page

D.1-4 Tier 2 SSTLs and CAU 476 Results for Hazardous Constituents of Diesel............ D-8

D.1-5 Tier 2 SSTLs and CAU 476 Results for Radiological Constituents ....................... D-9 


\section{List of Acronyms and Abbreviations}

Am

ARL

ASTM

bgs

CADD

CAI

CAIP

CAS

CAU

$\mathrm{cm}^{3} / \mathrm{g}$

Co

COC

COPC

CR

Cs

CSM

$\mathrm{CZ}$

DOE

DQA

DQI

DQO

DRO

DTRA

EPA

FAL
Americium

Air Resources Laboratory

American Society for Testing and Materials

Below ground surface

Corrective Action Decision Document

Corrective Action Investigation

Corrective Action Investigation Plan

Corrective Action Site

Corrective Action Unit

Cubic centimeters per gram

Cobalt

Contaminant of concern

Contaminant of potential concern

Closure Report

Cesium

Conceptual site model

Contaminated Zone

U.S. Department of Energy

Data quality assessment

Data quality indicator

Data quality objective

Diesel-range organics

Defense Threat Reduction Agency

U.S. Environmental Protection Agency

Final action level 


\section{List of Acronyms and Abbreviations (continued)}

\begin{tabular}{|c|c|}
\hline FFACO & Federal Facility Agreement and Consent Order \\
\hline FGR & Federal Guidance Report \\
\hline $\mathrm{ft}$ & Foot \\
\hline $\mathrm{g} / \mathrm{cm}^{3}$ & Grams per cubic centimeter \\
\hline $\mathrm{g} / \mathrm{m}^{3}$ & Grams per cubic meter \\
\hline $\mathrm{g} / \mathrm{yr}$ & Grams per year \\
\hline $\mathrm{hr} / \mathrm{yr}$ & Hours per year \\
\hline $\mathrm{kg} /$ day & Kilograms per day \\
\hline $\mathrm{kg} / \mathrm{yr}$ & Kilograms per year \\
\hline $\mathrm{L} /$ day & Liters per day \\
\hline $\mathrm{L} / \mathrm{yr}$ & Liters per year \\
\hline $\mathrm{m}$ & Meter \\
\hline $\mathrm{m} / \mathrm{sec}$ & Meters per second \\
\hline $\mathrm{m} / \mathrm{yr}$ & Meters per year \\
\hline $\mathrm{m}^{2}$ & Square meter \\
\hline $\mathrm{m}^{3} / \mathrm{h}$ & Cubic meters per hour \\
\hline $\mathrm{m}^{3} / \mathrm{yr}$ & Cubic meters per year \\
\hline $\mathrm{mg} / \mathrm{kg}$ & Milligrams per kilogram \\
\hline $\mathrm{M} \& \mathrm{O}$ & Management and operating \\
\hline mrem & Millirem \\
\hline $\mathrm{mrem} / \mathrm{yr}$ & Millirem per year \\
\hline MS & Matrix spike \\
\hline MSD & Matrix spike duplicate \\
\hline N/A & Not applicable \\
\hline NAC & Nevada Administrative Code \\
\hline NCRP & National Council on Radiation Protection and Measurements \\
\hline
\end{tabular}




\section{List of Acronyms and Abbreviations (continued)}

$\begin{array}{ll}\text { ND } & \text { Nondetect } \\ \text { NDEP } & \text { Nevada Division of Environmental Protection } \\ \text { NNSA/NSO } & \begin{array}{l}\text { U.S. Department of Energy, National Nuclear Security Administration } \\ \text { Nevada Site Office }\end{array} \\ \text { NTS } & \text { Nevada Test Site } \\ \text { ORERP } & \text { Off-Site Radiation Exposure Review Project } \\ \text { PAL } & \text { Preliminary action level } \\ \text { Pb } & \text { Lead } \\ \text { pCi/g } & \text { Picocuries per gram } \\ \text { PRG } & \text { Preliminary remediation goal } \\ \text { Pu } & \text { Plutonium } \\ \text { QA } & \text { Quality assurance } \\ \text { QAPP } & \text { Quality Assurance Project Plan } \\ \text { QC } & \text { Quality control } \\ \text { Ra } & \text { Radium } \\ \text { RCRA } & \text { Resource Conservation and Recovery Act } \\ \text { RESRAD } & \text { Residual Radioactive } \\ \text { RPD } & \text { Relative percent difference } \\ \text { RT } & \text { Regulatory threshold } \\ \text { Sb } & \text { Antimony } \\ \text { SNJV } & \text { Stoller-Navarro Joint Venture } \\ \text { SORD } & \text { Special Operations and Research Division } \\ \text { SSTL } & \text { Site-specific target level } \\ \text { SVOC } & \text { Semivolatile organic compound } \\ \text { TEDE } & \text { Total effective dose equivalent } \\ \text { Th } & \text { Thorium } \\ & \\ \text { R } & \\ \text { R } & \end{array}$




\section{List of Acronyms and Abbreviations (continued)}

$\mathrm{Tl}$

TPH Total petroleum hydrocarbons

UCL Upper confidence level

VOC Volatile organic compound

yr $\quad$ Year

/yr Per year

$\mu \mathrm{g} / \mathrm{kg} \quad$ Micrograms per kilogram 


\section{Executive Summary}

This Corrective Action Decision Document (CADD)/Closure Report (CR) was prepared by the Defense Threat Reduction Agency (DTRA) for Corrective Action Unit (CAU) 476, Area 12 T-Tunnel Muckpile. This CADD/CR is consistent with the requirements of the Federal Facility Agreement and Consent Order (FFACO) agreed to by the State of Nevada, the U.S. Department of Energy, and the U.S. Department of Defense. Corrective Action Unit 476 is comprised of one Corrective Action Site (CAS):

\section{- 12-06-02, Muckpile}

The purpose of this CADD/CR is to provide justification and documentation supporting the recommendation for closure in place with use restrictions for CAU 476. To support this recommendation, a corrective action investigation (CAI) was performed in April and May 2000. The purpose of the CAI was to fulfill the following data needs as defined during the Data Quality Objective (DQO) process:

- Determine whether contaminants of concern (COCs) are present.

- If COCs are present, determine their nature and extent.

- Obtain sufficient information to determine appropriate corrective action.

The CAU 476 dataset from the CAI was evaluated based on the data quality indicator parameters. This evaluation demonstrated the quality and acceptability of the dataset for use in fulfilling the DQO data needs (Appendix C of this document).

Analytes detected during the CAI were evaluated against final action levels (FALs) established in this document. Tier 2 FALS were determined for the hazardous constituents of total petroleum hydrocarbons (TPH)-diesel-range organics (DRO) and the radionuclide cesium (Cs)-137. Tier 2 FALs were calculated using site-specific information. The hazardous constituents of TPH-DRO were compared to the preliminary action levels (PALs) defined in the Corrective Action Investigation Plan (CAIP), and because none of the individual PALs were exceeded, the PALs became the FALs and TPH-DRO is not a COC for the CAS. The radionuclide FALs were calculated using the Residual Radioactive (RESRAD) computer code (version 6.3) for the occasional reuse scenario. The RESRAD calculation determined the required activities for all radionuclides based on their relative abundance at the site that together would sum to an exposure dose of 25 millirem per year to a site receptor. Based on the field investigation, none of the contaminants were determined to be present at concentrations 
exceeding their corresponding FALs. As specified in the CAIP, Cs-137 and TPH-DRO were used to determine whether the site was adequately characterized. The analytical results for Cs-137 and TPH-DRO were entered into the SW-846 formula to determine whether the site had been characterized to the 90 percent confidence level. Enough samples were collected to characterize the site with respect to those constituents to the 90 percent confidence level. The sampling was adequate to define the lateral and vertical extent of Cs-137.

Based on the data and risk evaluations, the DQO data needs presented in the CAIP were met, and the data accurately represent the radiological and chemical risk present at CAU 476. Based on the results of the CAI data evaluation, it was determined that closure in place with use restrictions is the appropriate corrective action for CAU 476 and that use restrictions will effectively control exposure to future land users. This is based on the fact that even though the site is contaminated with Cs-137 as described above, this remote, controlled access site poses only limited risk overall to public health and the environment. Therefore, DTRA provides the following recommendations:

- Close the Cs-137 in place at CAU 476 with use restriction.

- $\quad$ No further action for CAU 476.

- A Notice of Completion be issued to DTRA by the Nevada Division of Environmental Protection for closure of CAU 476.

- $\quad$ Move CAU 476 from Appendix III to Appendix IV of the FFACO. 


\section{$1.0 \quad$ Introduction}

This Corrective Action Decision Document (CADD)/Closure Report (CR) has been prepared for Corrective Action Unit (CAU) 476, Area 12 T-Tunnel Muckpile. The corrective action proposed in this document complies with the Federal Facility Agreement and Consent Order (FFACO) (1996) that was agreed to by the State of Nevada, U.S. Department of Energy (DOE), and the U.S. Department of Defense (FFACO, 1996).

The T-Tunnel Muckpile is identified under FFACO classification as CAU 476, Area 12 T-Tunnel Muckpile. The CAU consists of one Corrective Action Site (CAS): 12-06-02 (Muckpile). The T-Tunnel Muckpile is located approximately 45 miles north of Mercury in Area 12 of the Nevada Test Site (NTS) (Figure 1-1).

This CADD/CR describes the corrective action that is selected as a result of the investigation activities and the rationale for its selection. The rationale consists of a justification for closure in place with use restrictions in accordance with Sections IV.8 and IV.11 of the FFACO (1996).

\section{$1.1 \quad$ Purpose}

The purpose of this CADD/CR is to provide justification for the closure of CAU 476 with use restrictions based on the results of the Corrective Action Investigation (CAI). The CAI was conducted in accordance with the Corrective Action Investigation Plan (CAIP) for Corrective Action Unit 476: Area 12 T-Tunnel Muckpile, Nevada Test Site (DTRA, 2000), which provides additional information on the history, planning, and scope of the investigation.

The T-Tunnel was used for six nuclear weapons effects tests and two high explosives tests between 1970 and 1997. The muckpile contains approximately 500,000 cubic yards of material consisting of mining debris (rock) generated during excavation of the tunnels and re-entry excavations following each test. Some construction waste such as steel, wood, cables, grout, and possibly small amounts of Resource Conservation and Recovery Act (RCRA)-regulated constituents and radionuclides were also deposited in the muckpile. One percent or less of the muckpile was expected to be composed of debris generated from re-entry operations (DNA, 1990). Additional information relating to the site history, planning, and scope of the investigation is presented in the CAIP (DTRA, 2000). 


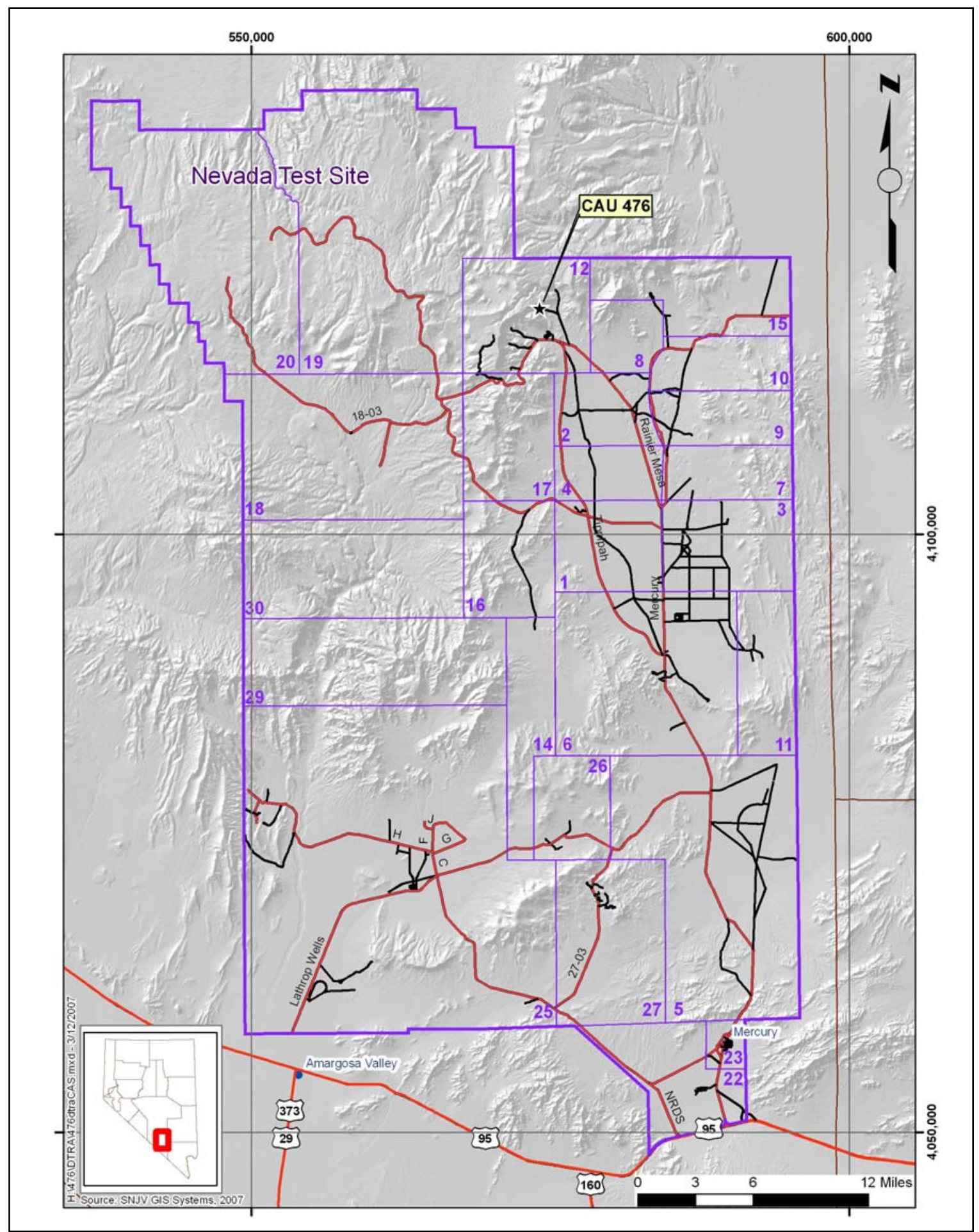

Figure 1-1

CAU 476 Location Map 


\subsection{Scope}

Subsequent to approval of the CAIP and completion of the CAI, the Nevada Division of Environmental Protection (NDEP) approved a risk-based approach for developing final action levels (FALs) to evaluate contaminant concentrations (Appenzeller-Wing, 2004). That approach was used to evaluate the potential hazards at CAU 476.

The initial evaluation of the contaminants of potential concern (COPCs) consisted of comparing individual sample and average concentrations to either the U.S. Environmental Protection Agency (EPA) Region 9 Preliminary Remediation Goals (PRGs) (EPA, 2004), or background concentrations as determined from background samples or specific documents that deal with the subject of background concentrations at the NTS. The new risk-based approach uses the PRGs and background concentrations as preliminary action levels (PALs). If the individual samples or average COPC concentrations exceed the PALs, they become contaminants of concern (COCs), and a risk analysis is conducted for those constituents. For the chemical constituents, the risk is calculated using the Risk Assessment Information System calculations (ORNL, 2005). For the radiological constituents, the Residual Radioactive (RESRAD) computer code (Yu, et al., 2001) is used to calculate the relative risk presented by the COCs.

The scope of this CADD/CR is to justify and recommend that closure in place with use restrictions is the appropriate action at CAU 476, Area 12 T-Tunnel Muckpile. To achieve this scope, the following actions were implemented:

- Evaluation of current site conditions, including the nature and extent of COCs.

- Closure in place with use restrictions to prevent exposure of industrial and construction workers to unacceptable risks.

The data quality objectives (DQOs) identified in the CAIP are as follows:

- Determine whether the muckpile contents are hazardous under RCRA, contain total petroleum hydrocarbons (TPH) contamination at levels exceeding the Nevada Administrative Code (NAC) limits, or contain radiological contamination exceeding background levels.

- If COCs are present, determine what further action will be needed.

The data quality indicators (DQIs) as defined in the Industrial Sites Quality Assurance Project Plan (QAPP) (DOE/NV, 1996) were achieved, and the DQOs established in the CAIP were met. 


\subsection{CADD/CR Contents}

This CADD/CR is divided into the following sections:

- Section 1.0 - Introduction: Summarizes the purpose, scope, and contents of this $\mathrm{CADD} / \mathrm{CR}$.

- Section 2.0 - CAI Summary: Summarizes the investigation field activities, the results of the investigation, and the DQO assessment.

- Section 3.0 - Recommendation: States why no further action is required.

- Section 4.0 - References: Lists all documents referenced in the CADD/CR.

- Appendix A - Corrective Action Investigation Report for CAU 476, Area 12 T-Tunnel Muckpile, Nevada Test Site

- Appendix B - Data Quality Objective Process and Methodology

- Appendix C - Data Assessment

- Appendix D - Risk Assessment for CAU 476

- Appendix E - Closure Summary

All work was performed in accordance with the following documents:

- Corrective Action Investigation Plan for Corrective Action Unit 476: Area 12 T-Tunnel Muckpile, Nevada Test Site, Rev. 0 (DTRA, 2000).

- Industrial Sites Quality Assurance Project Plan, Nevada Test Site, Nevada, Rev. 0 (DOE/NV, 1996)

- Federal Facility Agreement and Consent Order (FFACO, 1996) 


\subsection{Corrective Action Investigation Summary}

The following sections describe and summarize the results of the CAI activities conducted at CAU 476. For detailed CAI results, refer to Appendix A.

\section{$2.1 \quad$ Investigation Activities}

Between April 10 and May 2, 2000, CAI activities were performed at the T-Tunnel Muckpile as set forth in the CAIP (DTRA, 2000). The purpose of the CAI was to determine whether or not the T-Tunnel Muckpile and/or the underlying native soils contain COCs and, if so, determine the extent of the COCs and whether they pose an unacceptable risk to human health and/or the environment. As outlined in the CAIP (DTRA, 2000), the following tasks were performed:

- Background sampling - Three locations were identified and sampled using hand tools. These samples were analyzed for RCRA metals and radionuclides.

- Surface Sampling - Eight locations were selected to characterize the surface/shallow subsurface of the muckpile (less than 5 feet [ft]). Seven boreholes were drilled to a depth of $5 \mathrm{ft}$. A continuous core was extracted from each borehole. Each core was field screened for volatile organic compounds (VOCs) and radionuclides, and the portion(s) of the core with the highest field-screening results was collected for laboratory analysis. If no portion of the core exhibited elevated field-screening results, the interval between 0.5 and $1.5 \mathrm{ft}$ was collected and submitted for laboratory analysis. Because one location was too close to the edge of the T-Tunnel Muckpile to safely drill, it was excavated by hand. Nine surface/shallow subsurface samples were collected from these locations and submitted for VOCs, semivolatile organic compounds (SVOCs), RCRA metals, $\mathrm{TPH}-$ diesel-range organics (DRO), and radionuclide analyses.

- Muckpile Contents/Underlying Soils Sampling - Twenty boreholes were drilled to characterize the subsurface of the muckpile from $5 \mathrm{ft}$ below ground surface to 2 to $5 \mathrm{ft}$ into the native soil under the muckpile and to determine whether any of the COCs have migrated into the underlying native soil. The boreholes provided samples for analysis of the muckpile contents and the native soil beneath the muckpile. A continuous core was extracted from each borehole and field screened, and 42 samples were submitted for VOC, SVOC, RCRA metals, TPH-DRO, and radionuclide analyses. One sample was collected at the bottom of each borehole to represent the native materials beneath the muckpile, and 20 samples were collected at random depths to represent the muckpile contents.

The conceptual site model (CSM) postulated that the majority of the muckpile does not contain COPCs (less than 1 percent), and if any COPCs are present, they are probably located in isolated locations and not present throughout the muck. The areas most likely to be affected are the areas 
where petroleum compounds were used for equipment maintenance activities, possibly resulting in releases to the surface and shallow subsurface soils. The potential also exists for the presence of radionuclides in the muckpile as a result of disposal of re-entry material from the tunnel. These releases, if present, were anticipated to have limited lateral and vertical extent. The CSM also stated that it is possible, but unlikely, that the native soil beneath the muckpile has been impacted by downward migration of COPCs. The results of the CAI showed that there is localized contamination with radionuclides in the T-Tunnel Muckpile. The CAI also demonstrated that no contaminants are leaching into the native materials below the muckpile. Based on these facts, the CSM was shown to be valid.

\section{$2.2 \quad$ Results}

The following is a summary of the data obtained during the CAI.

\subsubsection{Summary of Analytical Data}

The CAI analytical results (Appendix A) indicate the following:

- Volatile organic compounds and SVOCs were detected in muckpile samples at concentrations that did not exceed the action levels outlined in the CAIP (DTRA, 2000).

- The TPH-diesel concentrations that exceeded the NDEP-established action level of 100 milligrams per kilogram (mg/kg) (DTRA, 2000) were identified at locations \#18, bh-s6, bh-s7, and bh-s8 in the muckpile (Figure 2-1). All locations were shallow surface/subsurface ( 0 to $3.5 \mathrm{ft}$ ) with concentrations ranging from $110 \mathrm{mg} / \mathrm{kg}$ to $1,200 \mathrm{mg} / \mathrm{kg}$. However, there were no hazardous constituents of TPH-DRO detected in the samples that exceeded their respective PALs (Appendix D).

- Radionuclide results were compared to soil samples taken from undisturbed locations in the Western and Southwestern United States (U.S. Ecology and Atlan-Tech, Inc., 1991; and McArthur and Miller, 1989). Thallium (Tl)-208, lead (Pb)-212, and Pb-214 were detected in several samples but the concentrations are not considered to be statistically significant when compared to background (Orchard and Alderson, 2000). In addition, these isotopes are naturally occurring and are not produced during weapons testing. Cesium (Cs)-137, a product of fission, was detected in surface samples TS-S1-0.5, TS-S2-0.5, TS-S4-0.5, TS-S5-0.5, and TS-S6-0.5 at levels that exceeded the background concentration. For the samples, TS indicates T-Tunnel soil; S1, S2, S4, S5, and S6 refer to the bh-location (Figure 2-1); and the last numbers are the depth below ground surface. Americium (Am)-241, cobalt (Co)-60, antimony (Sb)-125, and plutonium (Pu)-238/239 were also found within the muckpile at shallow surface locations and in the shallow background samples at concentrations exceeding that from fallout found in undisturbed background locations that are not in the vicinity of the T-Tunnel Muckpile. 


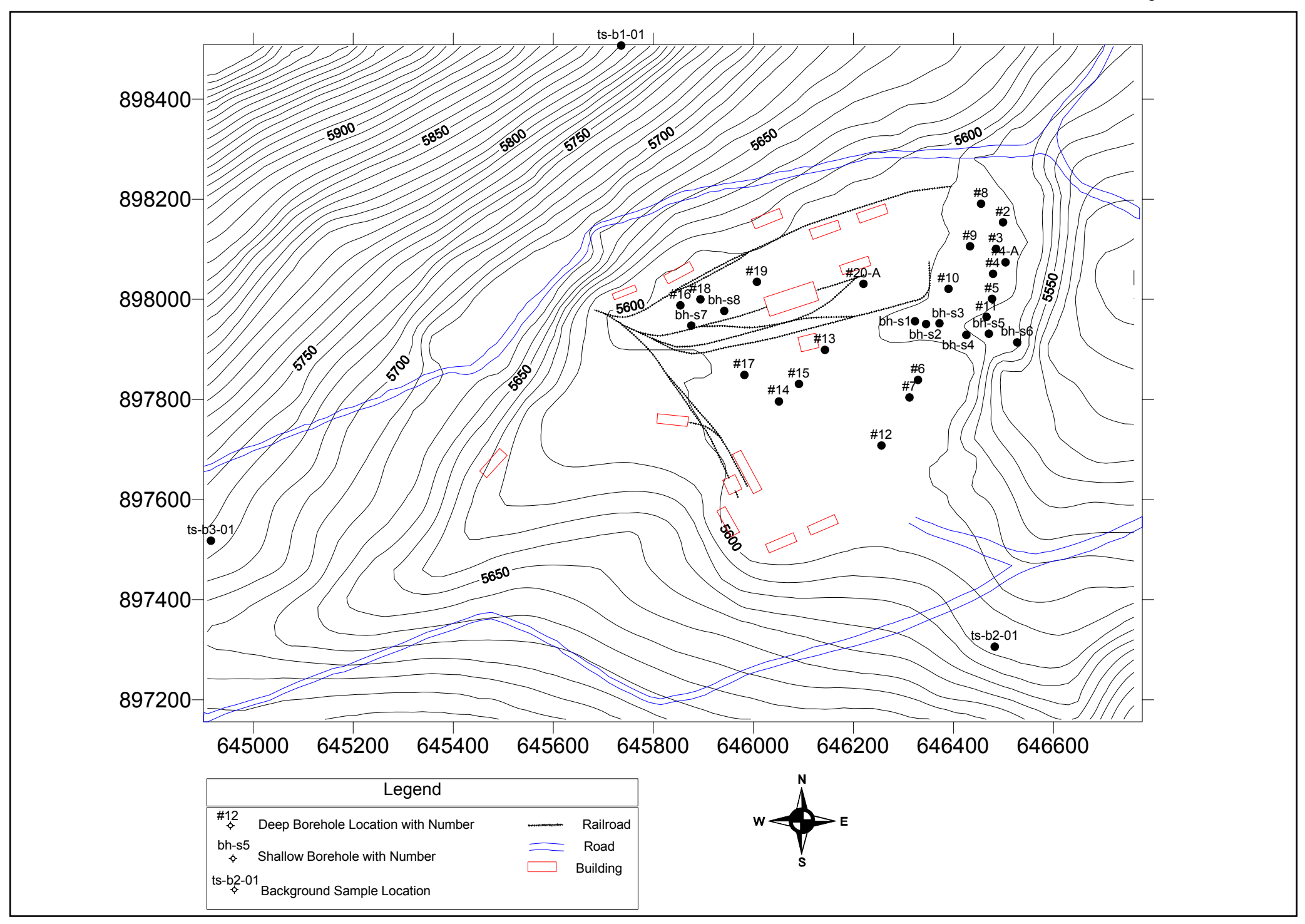

Figure 2-1

T-Tunnel Muckpile Sample Locations 
- No COCs above the action levels as defined in the CAIP (DTRA, 2000) or Appendix D were detected in the native soil below the muckpile.

Details of the methods used and results found during the CAI are presented in Appendix A. A statistical analysis of the analytical data from the CAI has demonstrated that the number of samples taken was sufficient and resulted in a greater than 90 percent confidence level that the mean concentrations are representative of the muckpile. Based on these results, the nature and extent of COCs at CAU 476 have been adequately identified and were used to develop and evaluate corrective action alternatives.

\subsubsection{Muckpile (CAS 12-06-02)}

Except as noted above, none of the chemical constituents found in the muckpile were detected above the PALs, so the PALs were identified as the FALs for those constituents. For TPH-DRO, the constituent that exceeded its PAL, a site-specific target level (SSTL) for the occasional use scenario was calculated, which then became the FAL for that constituent. The SSTL for TPH-DRO is the PAL for the hazardous constituents found in TPH-DRO. None of the hazardous constituent concentrations exceed their PAL. The maximum concentration of each detected chemical constituent within the muckpile is listed in Table 2-1. A more detailed discussion of the constituents and the determination of the FALs is provided in Appendix D.

Table 2-1

Maximum Reported Chemical Values for Muck in CAS 12-06-02, Muckpile (Page 1 of 2)

\begin{tabular}{|c|c|c|c|c|}
\hline Contaminant & $\begin{array}{c}\text { Result } \\
(\mathrm{mg} / \mathrm{kg})\end{array}$ & Sample No. & $\begin{array}{c}\text { Depth } \\
\text { (ft bgs) }\end{array}$ & $\begin{array}{c}\text { Final Action } \\
\text { Level }(\mathrm{mg} / \mathrm{kg})\end{array}$ \\
\hline 1,1-Dichloroethene & 0.0013 & TS-13-14 & $13.5-14.5$ & $410^{a}$ \\
\hline 1,2,3-Trichlorobenzene & 0.0014 & TS-20-20 & $19.5-20.5$ & $220^{a}$ \\
\hline 1,2,4-Trimethylbenzene & 0.001 & TS-13-14 & $13.5-14.5$ & $170^{\mathrm{a}}$ \\
\hline 4-Methyl-2-Pentanone & 0.0027 & TS-18-3.0 & $2.5-3.5$ & $47,000^{a}$ \\
\hline Acetone & 0.021 & TS-09-30 & $29.5-30.5$ & $54,000^{a}$ \\
\hline Arsenic & 13 & TS-13-14 & $13.5-14.5$ & $23^{b}$ \\
\hline Barium & 3,300 & TS-04A-58 & $57.5-58.5$ & $67,000^{a}$ \\
\hline Cadmium & 0.4 & TS-09-30 & $29.5-30.5$ & $450^{a}$ \\
\hline Chromium & 11 & TS-09-35 & $34.5-35.5$ & $450^{\mathrm{a}}$ \\
\hline Diesel-Range Organics & 330 & TS-18-3.0 & $2.5-3.5$ & See footnote ${ }^{c}$ \\
\hline Lead & 210 & TS-13-14 & $13.5-14.5$ & $800^{\mathrm{a}}$ \\
\hline Mercury & 0.079 & TS-12-14 & $13.5-14.5$ & $310^{a}$ \\
\hline
\end{tabular}


Table 2-1

\section{Maximum Reported Chemical Values for} Muck in CAS 12-06-02, Muckpile

(Page 2 of 2)

\begin{tabular}{|c|c|c|c|c||}
\hline \hline Contaminant & $\begin{array}{c}\text { Result } \\
\mathbf{( m g / k g})\end{array}$ & Sample No. & $\begin{array}{c}\text { Depth } \\
\mathbf{( f t} \mathbf{b g s})\end{array}$ & $\begin{array}{c}\text { Final Action } \\
\text { Level } \mathbf{( m g} / \mathbf{k g})\end{array}$ \\
\hline \hline Naphthalene & 0.0016 & TS-20-20 & $19.5-20.5$ & $190^{\mathrm{a}}$ \\
\hline N-Butylbenzene & 0.00088 & TS-20-20 & $19.5-20.5$ & $240^{\mathrm{a}}$ \\
\hline Selenium & 4.1 & TS-09-30 & $29.5-30.5$ & $5,100^{\mathrm{a}}$ \\
\hline Silver & 2.4 & TS-09-30 & $29.5-30.5$ & $5,100^{\mathrm{a}}$ \\
\hline Toluene & 0.0009 & TS-18-3.0 & $2.5-3.5$ & $520^{\mathrm{a}}$ \\
\hline Trichloroethene & 0.00076 & TS-12-14 & $13.5-12.5$ & $0.11^{\mathrm{a}}$ \\
\hline Trichlorotrifluoroethane & 0.0011 & TS-06-12 & $11.5-12.5$ & $77,900^{\mathrm{a}}$ \\
\hline
\end{tabular}

${ }^{\mathrm{a}}$ Final action level based on Region 9 Preliminary Remediation Goals (PRGs) (EPA, 2004).

${ }^{\mathrm{b}} \mathrm{Nevada}$ Test Site background plus two standard deviations.

${ }^{\mathrm{c}}$ Final action level for TPH-DRO is the preliminary action levels for the hazardous constituents found in TPH-DRO.

$\mathrm{DRO}=$ Diesel-range organics

$\mathrm{ft}$ bgs $=$ Feet below ground surface

$\mathrm{mg} / \mathrm{kg}=$ Milligrams per kilogram

$\mathrm{TPH}=$ Total petroleum hydrocarbons

None of the radionuclides found in the shallow soils on the muckpile exceeded the PALs as defined in the CAIP (DTRA, 2000), so the PALs for those radionuclides are identified as the FALs. The maximum concentration of each detected radionuclide found in the muckpile at this CAS is listed in Table 2-2.

Table 2-2

Maximum Reported Radiological Values for Muck in CAS 12-06-02, Muckpile

\begin{tabular}{|c|c|c|c|c||}
\hline Contaminant & $\begin{array}{c}\text { Result } \\
\mathbf{( p C i / g )}\end{array}$ & Sample No. & $\begin{array}{c}\text { Depth } \\
\text { (ft bgs) }\end{array}$ & $\begin{array}{c}\text { Final Action } \\
\text { Level (pCi/g) }\end{array}$ \\
\hline \hline Actinium-228 & 3.92 & TS-03-09 & $8.5-9.5$ & $5^{\text {a }}$ \\
\hline Bismuth-212 & 3.2 & TS-04A-58 & $57.5-58.5$ & $5^{\text {a }}$ \\
\hline Bismuth-214 & 1.8 & TS-20-20 & $19.5-20.5$ & $5^{\text {a }}$ \\
\hline Cesium-137 & 0.58 & TS-05-24 & $23.5-24.5$ & $12.2^{\text {a }}$ \\
\hline Lead-212 & 3.54 & TS-02-8.5 & $8.0-9.0$ & $5^{\text {a }}$ \\
\hline Lead-214 & 2.08 & TS-03-09 & $8.5-9.5$ & $5^{\text {a }}$ \\
\hline Thallium-208 & 1.15 & QTS-05-24 & $23.5-24.5$ & $5^{\text {a }}$ \\
\hline
\end{tabular}

${ }^{\mathrm{a}}$ Final action level based on background or the National Council on Radiation Protection and Measurements Report No. 129 recommended screening limits for construction, commercial, and industrial land-use scenario (NCRP, 1999) scaled from 25- to 15-millirem-per-year dose and the generic guidelines for residual concentration of radionuclides in DOE Order 5400.5 (DOE, 1993).

$\mathrm{ft}$ bgs $=$ Feet below ground surface $\mathrm{pCi} / \mathrm{g}=$ Picocuries per gram 


\subsubsection{Native Material Under the Muckpile}

None of the chemical constituents found in the native material under the muckpile exceeded the PALs as identified in the CAIP (DTRA, 2000), so the PALs are identified as the FALs. The maximum concentration of each detected chemical contaminant found in the native material at this CAS is listed in Table 2-3.

Table 2-3

Maximum Reported Chemical Values for Native Material Under CAS 12-06-02 Muckpile

\begin{tabular}{|c|c|c|c|c||}
\hline Contaminant & $\begin{array}{c}\text { Result } \\
\mathbf{( m g / k g})\end{array}$ & Sample No. & $\begin{array}{c}\text { Depth } \\
\text { (ft bgs) }\end{array}$ & $\begin{array}{c}\text { Final Action } \\
\text { level }(\mathbf{m g} / \mathbf{k g})\end{array}$ \\
\hline 1,1-Dichloroethene & 0.0013 & TS-14-24 & $23.5-24.5$ & $410^{\mathrm{a}}$ \\
\hline 4-Methyl-2-Pentanone & 0.002 & TS-14-24 & $23.5-24.5$ & $47,000^{\mathrm{a}}$ \\
\hline Acetone & 0.019 & TS-04A-68.5 & $68-69$ & $54,000^{\mathrm{a}}$ \\
\hline Arsenic & 7.2 & TS-18-20 & $19.5-20.5$ & $23^{\mathrm{b}}$ \\
\hline Barium & 200 & TS-15-29 & $28.5-29.5$ & $67,000^{\mathrm{a}}$ \\
\hline Benzoic Acid & 0.27 & TS-18-20 & $19.5-20.5$ & $100,000^{\mathrm{a}}$ \\
\hline Chromium & 10 & TS-15-29 & $28.5-29.5$ & $450^{\mathrm{a}}$ \\
\hline Diesel-Range Organics & 47 & TS-04-63.5 & $63-64$ & $100^{\mathrm{c}}$ \\
\hline Lead & 18 & TS-06-47.5 & $47-48$ & $800^{\mathrm{a}}$ \\
\hline Selenium & 1.5 & TS-13-41 & $40.5-41.5$ & $5,100^{\mathrm{a}}$ \\
\hline Silver & 0.8 & TS-18-20 & $19.5-20.5$ & $5,100^{\mathrm{a}}$ \\
\hline Trichlorotrifluoroethane & 0.00096 & TS-06-47.5 & $47-48$ & $77,900^{\mathrm{a}}$ \\
\hline
\end{tabular}

${ }^{\mathrm{a}}$ Final action level based on Region 9 Preliminary Remediation Goals (PRGs) (EPA, 2004).

${ }^{\mathrm{b}}$ Nevada Test Site background plus two standard deviations.

${ }^{\mathrm{C} N e v a d a}$ Administrative Code 445A.2272 (NAC, 2003b)

$\mathrm{ft}$ bgs $=$ Feet below ground surface

$\mathrm{mg} / \mathrm{kg}=$ Milligrams per kilogram

None of the radionuclides found in the native material under the muckpile exceeded the PALs as defined in the CAIP (DTRA, 2000), so the PALs for those radionuclides were established as the FALs. The maximum concentration of each detected radionuclide found in the native material under the pad at this CAS is listed in Table 2-4. 
Table 2-4

Maximum Reported Radiological Values for Native Material Under CAS 12-06-02 Muckpile

\begin{tabular}{|c|c|c|c|c||}
\hline Contaminant & $\begin{array}{c}\text { Result } \\
(\mathbf{p C i} / \mathbf{g})\end{array}$ & Sample No. & $\begin{array}{c}\text { Depth } \\
\text { (ft bgs) }\end{array}$ & $\begin{array}{c}\text { Final Action } \\
\text { Level (pCi/g) }\end{array}$ \\
\hline \hline Actinium-228 & 3.53 & TS-04A-68.5 & $68-69$ & $5^{\mathrm{a}}$ \\
\hline Americium-241 & 0.62 & TS-04-63.5 & $63-64$ & $12.7^{\mathrm{a}}$ \\
\hline Bismuth-214 & 2.43 & TS-17-25 & $24.5-25.5$ & $5^{\mathrm{a}}$ \\
\hline Lead-212 & 3.54 & TS-02-56.5 & $56-57$ & $5^{\mathrm{a}}$ \\
\hline Lead-214 & 2.87 & TS-17-25 & $24.5-25.5$ & $5^{\mathrm{a}}$ \\
\hline Thallium-208 & 1.06 & TS-03-61.5 & $61-62$ & $5^{\mathrm{a}}$ \\
\hline
\end{tabular}

${ }^{\mathrm{a}}$ Final action level based on background or the National Council on Radiation Protection and Measurements Report No. 129 recommended screening limits for construction, commercial, and industrial land-use scenario (NCRP, 1999) scaled from 25- to 15-millirem-per-year dose and the generic guidelines for residual concentration of radionuclides in DOE Order 5400.5 (DOE, 1993).

$\mathrm{ft}$ bgs $=$ Feet below ground surface

$\mathrm{pCi} / \mathrm{g}=$ Picocuries per gram

\subsubsection{Shallow Soils on the Muckpile}

None of the chemical constituents found in the shallow soils on the muckpile exceeded the PALs as identified in the CAIP (DTRA, 2000) except for TPH-DRO, so the PALs are identified as the FALs for those constituents. The maximum concentration of each detected chemical contaminant found in the shallow soils at this CAS is listed in Table 2-5. The TPH-DRO SSTL was calculated as explained in Section 2.2.1.1.

Table 2-5

Maximum Reported Chemical Values for Shallow Soils at CAS 12-06-02 Muckpile (Page 1 of 2)

\begin{tabular}{|c|c|c|c|c||}
\hline Contaminant & $\begin{array}{c}\text { Result } \\
\mathbf{( m g / k g )}\end{array}$ & Sample No. & $\begin{array}{c}\text { Depth } \\
\mathbf{( f t ~ b g s )}\end{array}$ & $\begin{array}{c}\text { Final Action } \\
\text { Level }(\mathbf{m g} / \mathbf{k g})\end{array}$ \\
\hline \hline Acetone & 0.025 & TS-S2-0.5 & $0-1.0$ & $54,000^{\mathrm{a}}$ \\
\hline Arsenic & 5.8 & TS-S1-2.5 & $2.0-3.0$ & $23^{\mathrm{b}}$ \\
\hline Barium & 4,500 & TS-S3-01 & $0.5-1.5$ & $67,000^{\mathrm{a}}$ \\
\hline Cadmium & 0.24 & TS-S1-2.5 & $2.0-3.0$ & $450^{\mathrm{a}}$ \\
\hline Chromium & 12 & TS-S1-2.5 & $2.0-3.0$ & $450^{\mathrm{a}}$ \\
\hline Diesel-Range Organics & 1,200 & TS-S7-01 & $0.5-1.5$ & See footnote $^{\mathrm{c}}$ \\
\hline Di-N-Buthyl Phthalate & 26 & TS-S1-0.5 & $0-1.0$ & $62,000^{\mathrm{a}}$ \\
\hline Lead & 63 & TS-S4-0.5 & $0-1.0$ & $800^{\mathrm{a}}$ \\
\hline
\end{tabular}


Table 2-5

Maximum Reported Chemical Values for Shallow Soils at CAS 12-06-02 Muckpile (Page 2 of 2)

\begin{tabular}{|c|c|c|c|c||}
\hline Contaminant & $\begin{array}{c}\text { Result } \\
\mathbf{( m g / k g )}\end{array}$ & Sample No & $\begin{array}{c}\text { Depth } \\
\mathbf{( f t ~ b g s )}\end{array}$ & $\begin{array}{c}\text { Final Action } \\
\text { Level } \mathbf{( m g / k g})\end{array}$ \\
\hline \hline Selenium & 0.61 & TS-S4-0.5 & $0-1.0$ & $5,100^{\mathrm{a}}$ \\
\hline Silver & 0.32 & TS-S1-2.5 & $2.0-3.0$ & $5,100^{\mathrm{a}}$ \\
\hline
\end{tabular}

${ }^{a}$ Final action level based on Region 9 Preliminary Remediation Goals (PRGs) (EPA, 2004).

${ }^{\mathrm{b}} \mathrm{Nevada}$ Test Site background plus two standard deviations.

${ }^{c}$ Final action level for TPH-DRO is the preliminary action levels for the hazardous constituents found in TPH-DRO.

DRO = Diesel-range organics

$\mathrm{ft} \mathrm{bgs}=$ Feet below ground surface

$\mathrm{mg} / \mathrm{kg}=$ Milligrams per kilogram

$\mathrm{TPH}=$ Total petroleum hydrocarbons

None of the radionuclides found in the shallow soils on the muckpile were detected above their PALs except for Cs-137, so the PALs were identified as the FALs for those radionuclides. For Cs-137, which did exceed the PAL, a SSTL was calculated using RESRAD (Yu et al., 2001), and that calculation then became the FAL for that constituent. The maximum concentration of each detected radionuclide at this CAS is listed in Table 2-6. A more detailed discussion of the radionuclides and the determination of the FALs is provided in Appendix D.

Table 2-6

Maximum Reported Radiological Values for Shallow Soils at CAS 12-06-02 Muckpile

(Page 1 of 2)

\begin{tabular}{|c|c|c|c|c||}
\hline Contaminant & $\begin{array}{c}\text { Result } \\
\mathbf{( p C i / g )}\end{array}$ & Sample No. & $\begin{array}{c}\text { Depth } \\
\mathbf{f f t} \mathbf{b g s})\end{array}$ & $\begin{array}{c}\text { Final Action } \\
\text { Level (pCi/g) }\end{array}$ \\
\hline \hline Actinium-228 & 3.9 & TS-S6-0.5 & $0-1.0$ & $5^{\mathrm{a}}$ \\
\hline Antimony-125 & 3 & TS-S1-0.5 & $0-1.0$ & $18.1^{\mathrm{a}}$ \\
\hline Bismuth-214 & 1.67 & TS-S7-01 & $0.5-1.5$ & $5^{\mathrm{a}}$ \\
\hline Cobalt-60 & 1.76 & TS-S6-0.5 & $0-1.0$ & $2.7^{\mathrm{a}}$ \\
\hline Cesium-137 & 382 & TS-S6-0.5 & $0-1.0$ & $373.6^{\mathrm{b}}$ \\
\hline Lead-212 & 3.27 & TS-S7-01 & $0.5-1.5$ & $5^{\mathrm{a}}$ \\
\hline Lead-214 & 2.3 & TS-S1-0.5 & $0-1.0$ & $5^{\mathrm{a}}$ \\
\hline Plutonium-238 & 0.91 & TS-S6-0.5 & $0-1.0$ & $13^{\mathrm{a}}$ \\
\hline Plutonium-239 & 2.87 & TS-S6-0.5 & $0-1.0$ & $12.7^{\mathrm{a}}$ \\
\hline
\end{tabular}


Table 2-6

Maximum Reported Radiological Values for Shallow Soils at CAS 12-06-02 Muckpile (Page 2 of 2)

\begin{tabular}{|c|c|c|c|c|}
\hline Contaminant & $\begin{array}{c}\text { Result } \\
(\mathbf{p C i} / \mathbf{g})\end{array}$ & Sample No. & $\begin{array}{c}\text { Depth } \\
\text { (ft bgs) }\end{array}$ & $\begin{array}{c}\text { Final Action } \\
\text { Level (pCi/g) }\end{array}$ \\
\hline \hline Thallium-208 & 0.64 & TS-S7-01 & $0.5-1.5$ & $5^{\text {a }}$ \\
\hline
\end{tabular}

${ }^{a}$ Final action level based on background or the National Council on Radiation Protection and Measurements Report No. 129 recommended screening limits for construction, commercial, and industrial land-use scenario (NCRP, 1999) scaled from 25- to 15-millirem-per-year dose and the generic guidelines for residual concentration of radionuclides in DOE Order 5400.5 (DOE, 1993)

${ }^{\mathrm{b}}$ Final action level based on RESRAD calculation (Yu et al., 2001) for remote scenario.

$\mathrm{ft}$ bgs $=$ Feet below ground surface

$\mathrm{pCi} / \mathrm{g}=$ Picocuries per gram

RESRAD = Residual Radioactive

\subsubsection{T-Tunnel Background}

Table 2-7 shows the maximum concentration of chemical constituents found in the background samples. Table 2-8 shows the maximum concentration of radionuclides found in the background samples.

Table 2-7

Maximum Reported Chemical Values for T-Tunnel Background

\begin{tabular}{|c|c|c|c|c||}
\hline Contaminant & $\begin{array}{c}\text { Result } \\
\mathbf{( m g / k g )}\end{array}$ & Sample No. & $\begin{array}{c}\text { Depth } \\
\mathbf{( f t ~ b g s )}\end{array}$ & $\begin{array}{c}\text { Final Action } \\
\text { Level } \mathbf{( m g / k g )}\end{array}$ \\
\hline \hline Arsenic & 3.8 & TS-B3-01 & $0.5-1.5$ & $23^{\mathrm{b}}$ \\
\hline Barium & 110 & TS-B3-01 & $0.5-1.5$ & $67,000^{\mathrm{a}}$ \\
\hline Cadmium & 0.088 & TS-B3-01 & $0.5-1.5$ & $450^{\mathrm{a}}$ \\
\hline Chromium & 6 & TS-B3-01 & $0.5-1.5$ & $450^{\mathrm{a}}$ \\
\hline Lead & 12 & TS-B2-01 & $0.5-1.5$ & $800^{\mathrm{a}}$ \\
\hline
\end{tabular}

${ }^{\mathrm{a}}$ Final action level based on Region 9 Preliminary Remediation Goals (PRGS) (EPA, 2004).

${ }^{\mathrm{b}} \mathrm{Nevada}$ Test Site background plus two standard deviations.

$\mathrm{ft}$ bgs $=$ Feet below ground surface $\mathrm{pCi} / \mathrm{g}=$ Picocuries per gram 
Table 2-8

Maximum Reported Radiological Values for T-Tunnel Background

\begin{tabular}{|c|c|c|c|c||}
\hline Contaminant & $\begin{array}{c}\text { Result } \\
\mathbf{( p C i / g )}\end{array}$ & Sample No. & $\begin{array}{c}\text { Depth } \\
\mathbf{( f t ~ b g s )}\end{array}$ & $\begin{array}{c}\text { Final Action } \\
\text { Level } \mathbf{( p C i / g )}\end{array}$ \\
\hline \hline Actinium-228 & 3.2 & TS-B2-01 & $0.5-1.5$ & $5^{\mathrm{a}}$ \\
\hline Americium-241 & 2.8 & TS-B2-01 & $0.5-1.5$ & $12.7^{\mathrm{a}}$ \\
\hline Bismuth-214 & 2.4 & TS-B2-01 & $0.5-1.5$ & $5^{\mathrm{a}}$ \\
\hline Cesium-137 & 13.5 & TS-B2-01 & $0.5-1.5$ & $373.6^{\mathrm{b}}$ \\
\hline Lead-212 & 2.76 & TS-B3-01 & $0.5-1.5$ & $5^{\mathrm{a}}$ \\
\hline Lead-214 & 1.59 & TS-B1-01 & $0.5-1.5$ & $5^{\mathrm{a}}$ \\
\hline Plutonium-238 & 1.03 & TS-B2-01 & $0.5-1.5$ & $13^{\mathrm{a}}$ \\
\hline Plutonium-239 & 9.6 & TS-B2-01 & $0.5-1.5$ & $12.7^{\mathrm{a}}$ \\
\hline Thallium-208 & 0.88 & TS-B1-01 & $0.5-1.5$ & $5^{\mathrm{a}}$ \\
\hline
\end{tabular}

${ }^{a}$ Final action level based on background or the National Council on Radiation Protection and Measurements Report No. 129 recommended screening limits for construction, commercial, and industrial land-use scenario (NCRP, 1999) scaled from 25- to 15-millirem-per-year dose and the generic guidelines for residual concentration of radionuclides in DOE Order 5400.5 (DOE, 1993)

${ }^{\mathrm{b}}$ Final action level based on RESRAD calculation (Yu et al., 2001) for remote use scenario.

$\mathrm{ft}$ bgs $=$ Feet below ground surface $\mathrm{pCi} / \mathrm{g}=$ Picocuries per gram RESRAD = Residual Radioactive

\subsubsection{Data Assessment Summary}

The data quality assessment (DQA) is presented in Appendix C and includes an evaluation of the DQIs to determine the degree of acceptability and usability of the data in the decision-making process. The DQO process ensures that the right type, quality, and quantity of data are available to support the resolution of those decisions at an appropriate level of confidence. Using both the DQO and DQA processes helps ensure that DQO decisions are sound and defensible.

The DQA process as presented in Appendix $\mathrm{C}$ is comprised of the following steps:

- Step 1 - Review DQOs and Sampling Design.

- Step 2 - Conduct a Preliminary Data Review.

- Step 3 - Select the Test.

- Step 4 - Verify the Assumptions.

- Step 5 - Draw Conclusions from the Data.

Sample locations that support the presence and/or extent of contamination at CAU 476 are shown in Appendix A and Figure 1-1. Based on the results of the DQA presented in Appendix C, the DQO requirements have been met, and the close in place with use restrictions 
corrective action alternative was selected as the closure alternative at CAU 476 (Area 12 T-Tunnel Muckpile). The DQA also determined that information generated during the investigation supports the CSM assumptions, and the data collected support the intended use in the decision-making process.

\subsection{Justification for No Further Action}

Use restrictions with no further corrective action is justified based on an evaluation of risk that ensures protection of the public and the environment in accordance with NAC 445A (NAC, 2003a), feasibility, and cost effectiveness. The selection of the corrective action was based on the resolution of the DQO decision statements and on a comparison of the analyte concentrations detected in CAI soil samples to the FALs defined in Section 2.3.1. Because the extent of the COCs is limited to the muckpile and the CAI demonstrated that there is no vertical migration through the muckpile into the underlying native material, the corrective action to close in place with administrative controls is justified at CAU 476. Appendix D presents an evaluation of risk associated with the recommended closure alternative.

\subsubsection{Final Action Levels}

The CAU 476 FALs are risk-based cleanup goals that, if met, will ensure that each release site will not pose an unacceptable risk to human health or the environment under the occasional use exposure scenario, and that the conditions at each site are in compliance with all applicable laws and regulations. The process described in this section to define and determine the FALs conforms to NAC Section 445A.2272 (NAC, 2003b), which lists the requirements for sites with soil contamination. For the evaluation of corrective actions, NAC Section 445A.22705 (NAC, 2003c) recommends the use of American Society for Testing and Materials (ASTM) Method E 1739-95 (ASTM, 1995) to "conduct an evaluation of the site, based on the risk it poses to public health and the environment, to determine the necessary remediation standards (i.e., FALs) or to establish that corrective action is not necessary."

The ASTM procedure defines three tiers (or levels) of evaluation involving increasingly sophisticated analyses as follows.

Tier 1 Evaluation - Sample results from source areas (highest concentrations) are compared to action levels based on generic (non-site-specific) conditions (i.e., the PALs established in the CAIP). The FALs may then be established as the Tier 1 action levels, or the FALs may be calculated using a Tier 2 evaluation. 
Tier 2 Evaluation - Conducted by calculating Tier 2 SSTLs using site-specific information as inputs to the same or similar methodology used to calculate Tier 1 action levels. The Tier 2 SSTLs are then compared to individual sample results from reasonable points of exposure (as opposed to the source areas as is done in Tier 1) on a point-by-point basis. Total TPH concentrations are not used for risk-based decisions under Tier 2 or Tier 3 . Rather, the individual hazardous constituents in TPH are compared to their SSTLs.

Alternatively, the Tier 2 risk-based corrective action process SSTLs may be compared to the predicted concentration or activity of the contaminant at the point of exposure based on attenuation from the source using relatively simplistic mathematical models. Points of exposure are defined as those locations at which an individual or population may come in contact with a $\mathrm{COC}$ originating from a CAS. If a Tier 2 evaluation is conducted, the calculations used to derive the SSTLs and the contaminant attenuation calculations will be provided as an appendix to the investigation report. If remediation to Tier 2 SSTLs is not practical, a Tier 3 evaluation may be conducted.

Tier 3 Evaluation - A Tier 3 evaluation is conducted by calculating SSTLs on the basis of more sophisticated risk analyses using methodologies described in ASTM Method E 1739-95 that consider site-, pathway-, and receptor-specific parameters. Tier 3 evaluation is much more complex than Tiers 1 and 2, because it may include additional site characterization, probabilistic evaluations, and sophisticated chemical fate/transport models. The Tier 3 SSTLs are then compared to the upper 95 percent confidence limit of the mean of sample results from reasonable points of exposure (as opposed to individual sample results as is done in Tier 2). Contaminant concentrations exceeding Tier 3 SSTLs require corrective action. If a Tier 3 evaluation is conducted, the calculations used to derive the SSTLs and the upper confidence limit of the means will be provided as an appendix to the investigation report.

A Tier 1 evaluation was conducted for all COPCs to determine whether contaminant levels satisfy the criteria for regulatory closure or warrant a more site-specific assessment. This was accomplished by comparing individual source area contaminant concentration results to the Tier 1 actions levels (the PALs established in the CAIP) on a point-by-point basis. The Tier 1 PALs were based on an industrial use scenario. 
The constituents detected at CAU 476 that exceeded Tier 1 action levels were:

- TPH-DRO

- Cs-137

The concentration of all constituents not listed above were below Tier 1 action levels, and the corresponding PALs were established as the Tier 1 FALs. The constituents that exceeded Tier 1 action levels were moved to a Tier 2 evaluation.

The Tier 2 evaluation of TPH-DRO compared the concentrations of the individual hazardous constituents of TPH-DRO to the Tier 1 action levels in the sample that exceeded for TPH-DRO. No hazardous constituents were found in the samples and therefore did not exceed Tier 1 action levels, so site-specific action levels were not calculated and TPH-DRO was eliminated as a COC. The PALs were established as the FALs for the hazardous constituents in TPH-DRO at CAU 476. The FALs are presented in Table 2-9. Additional details of the Tier 2 evaluation are provided in Appendix D.

Table 2-9

Tier 2 FALs and CAU 476 Results for Hazardous Constituents of Diesel (Page 1 of 2)

\begin{tabular}{|c|c|c|c|}
\hline CAS Number & Common Name & $\begin{array}{c}\text { Final Action Level } \\
(\mathbf{m g} / \mathbf{k g})\end{array}$ & $\begin{array}{c}\text { Maximum Reported } \\
\text { Value (mg/kg) }\end{array}$ \\
\hline \hline $108-67-8$ & 1,3,5-Trimethylbenzene & 70 & ND \\
\hline $91-57-6$ & 2-Methylnaphthalene ${ }^{\text {a }}$ & 190 & ND \\
\hline $56-55-3$ & Benzo(a)Anthracene & 2.1 & ND \\
\hline $71-43-2$ & Benzene & 1.4 & ND \\
\hline $50-32-8$ & Benzo(a)Pyrene & 0.21 & ND \\
\hline $100-41-4$ & Ethylbenzene & 400 & ND \\
\hline $91-20-3$ & Naphthalene & 190 & ND \\
\hline $108-88-3$ & Toluene & 520 & ND \\
\hline $1330-20-7$ & Total Xylene & ND \\
\hline $104-51-8$ & N-Butylbenzene & 420 & ND \\
\hline $103-65-1$ & N-Propylbenzene & 240 & ND \\
\hline $207-08-9$ & Benzo(k)Fluoranthene & 240 & ND \\
\hline $205-99-2$ & Benzo(b)Fluoranthene & 21 & ND \\
\hline $86-73-7$ & Fluorene & 21 & 26,000 \\
\hline $85-01-8$ & Phenanthrene & 100,000 & \\
\hline
\end{tabular}


Table 2-9

Tier 2 FALs and CAU 476 Results for Hazardous Constituents of Diesel (Page 2 of 2)

\begin{tabular}{|c|c|c|c||}
\hline CAS Number & Common Name & $\begin{array}{c}\text { Final Action Level } \\
\mathbf{( m g / k g )}\end{array}$ & $\begin{array}{c}\text { Maximum Reported } \\
\text { Value (mg/kg) }\end{array}$ \\
\hline \hline $206-44-0$ & Fluoranthene & 22,000 & ND \\
\hline $129-00-0$ & Pyrene & 29,000 & ND \\
\hline $218-01-9$ & Chrysene & 210 & ND \\
\hline $120-12-7$ & Anthracene & 100,000 & ND \\
\hline $191-24-2$ & Benzo(g,h,i)Perylene & 29,000 & ND \\
\hline
\end{tabular}

aUses PRG for naphthalene as surrogate

${ }^{\mathrm{b}}$ Total of $\mathrm{m}-$, o-, and $\mathrm{p}$-xylenes

CAS $=$ Chemical Abstracts Service

$\mathrm{mg} / \mathrm{kg}=$ Milligrams per kilograms

ND $=$ Nondetect

$P R G=$ Preliminary remediation goal

SSTL $=$ Site-specific target level

The Tier 2 evaluation for the radionuclides was conducted by entering site-specific radionuclide information and physical characteristics of the site into the RESRAD program to calculate the site-specific action levels. This calculated the site-specific activities needed to sum to an exposure dose of 25 millirem per year (mrem/yr) to a site receptor. These calculated concentrations were established as the FALs for each radionuclide at the CAS that exceeded a Tier 1 action level. The Tier 2 calculated FALs for the chemical and radiological constituents are presented in Table 2-10. Additional details of the Tier 2 evaluation are provided in Appendix D.

Table 2-10

Final Action Levels

\begin{tabular}{|c|c|c|c|}
\hline COPCs & Tier 1 FALs & Tier 2 FALs & Tier 3 FALs \\
\hline VOCs & PALs & $\mathrm{N} / \mathrm{A}$ & $\mathrm{N} / \mathrm{A}$ \\
\hline SVOCs & PALs & $\mathrm{N} / \mathrm{A}$ & $\mathrm{N} / \mathrm{A}$ \\
\hline RCRA metals & PALs & $\mathrm{N} / \mathrm{A}$ & $\mathrm{N} / \mathrm{A}$ \\
\hline TPH-DRO & N/A see Tier 2 & TPH-DRO hazardous constituent PALs & $\mathrm{N} / \mathrm{A}$ \\
\hline Radionuclides & $\begin{array}{l}\text { PALs except as } \\
\text { listed under Tier } 2\end{array}$ & Cs-137 $373.6 \mathrm{pCi} / \mathrm{g}$ & $\mathrm{N} / \mathrm{A}$ \\
\hline
\end{tabular}

COPC = Contaminant of potential concern $\mathrm{Cs}=$ Cesium

DRO = Diesel-range organics

$\mathrm{FAL}=$ Final action level

N/A = Not applicable

$\mathrm{PAL}=$ Preliminary action level
$\mathrm{pCi} / \mathrm{g}=$ Picocuries per gram RCRA = Resource Conservation and Recovery Act SVOC = Semivolatile organic compound $\mathrm{TPH}=$ Total petroleum hydrocarbons VOC $=$ Volatile organic compound 


\subsection{Recommendations}

The data generated by the CAI show that the FAL for Cs-137 was exceeded at CAU 476, Area 12 T-Tunnel Muckpile, and is the only COC present. Therefore, closure in place with use restrictions is considered the best option for closing this site. This recommendation is based on the fact that even though the FAL was exceeded for Cs-137, this remote, controlled access site poses only limited risk overall to public health and the environment. The future use of CAU 476 will be restricted from any activity unless concurrence is obtained from NDEP. The use restriction will prevent inadvertent contact with the COCs, and meets all applicable state and federal regulations for closure of the site.

In conclusion, the Defense Threat Reduction Agency (DTRA) requests that NDEP issue a Notice of Completion for this CAU and approval to move the CAU from Appendix III to Appendix IV of the FFACO. 


\subsection{References}

ASTM, see American Society for Testing and Materials.

American Society for Testing and Materials. 1995. Standard Guide for Risk-Based Corrective Action Applied at Petroleum Release Sites, ASTM E 1739-95 (Reapproved 2002).

Philadelphia, PA.

Appenzeller-Wing, J., U.S. Department of Energy, National Nuclear Security Administration Nevada Site Office. 2004. Letter to T.A. Maize entitled, "Submittal of Proposed Radiological Preliminary Action Levels (PALs) for the Industrial Sites Project," 15 January. Las Vegas, NV.

DNA, see Defense Nuclear Agency.

DOE, see U.S. Department of Energy.

DOE/NV, see U.S. Department of Energy/Nevada Operations Office.

DTRA, see Defense Threat Reduction Agency.

Defense Nuclear Agency. 1990. DNA Waste Management Document for DNA Activities at the Nevada Test Site. Las Vegas, NV: Test Directorate Nevada Operations Office.

Defense Threat Reduction Agency. 2000. Corrective Action Investigation Plan for Corrective Action Unit 476: Area 12 T-Tunnel Muckpile, Nevada Test Site, Rev. 0. Las Vegas, NV.

EPA, see U.S. Environmental Protection Agency.

FFACO, see Federal Facility Agreement and Consent Order.

Federal Facility Agreement and Consent Order. 1996 (as amended). Agreed to by the State of Nevada, the U.S. Department of Energy, and the U.S. Department of Defense.

McArthur, R.D., and F.L. Miller, Jr. 1989. Offsite Radiation Exposure Review Project. Phase II Soils Program, DOE/NV/10384-23. Las Vegas, NV: Desert Research Institute.

NAC, see Nevada Administrative Code.

NCRP, see National Council on Radiation Protection and Measurements.

National Council on Radiation Protection and Measurements. 1999. Recommended Screening Limits for Contaminated Surface Soil and Review of Factors Relevant to Site-Specific Studies. NCRP Report No. 129. Bethesda, MD

Nevada Administrative Code. 2003a. NAC 445A, "Water Controls.” Carson City, NV. 
Nevada Administrative Code. 2003b. NAC 445A.2272, "Contamination of Soil: Establishment of Action Levels." Carson City, NV.

Nevada Administrative Code. 2003c. NAC 445A.22705, "Contamination of Soil: Evaluation of Site by Owner or Operator; Review of Evaluation by Division.” Carson City, NV.

ORNL, see Oak Ridge National Laboratory.

Oak Ridge National Laboratory. 2005. "Risk Assessment Information System." As accessed at http://risk.lsd.ornl.gov/cgi-bin/prg/PRG_search.

Orchard, C.R. and S. Alderson. 2000. Memorandum to R. Deshler (ITLV) entitled, "Radioanalytical Data Review of Sample Results Associated with Corrective Action Unit 476 (T-Tunnel), Sample Delivery Groups 0004119, 0004125, 0004138, 0004146, 0004166, 0004183, 0004223, 0005007, 0005018, and 0005080,” 21 June. Las Vegas, NV: IT Corporation.

SNJV GIS Systems, see Stoller-Navarro Joint Venture Geographic Information Systems.

Stoller-Navarro Joint Venture Geographic Information Systems. 2007. ESRI Arc GIS Software.

U.S. Department of Energy. 1993. Radiation Protection of the Public and the Environment. DOE Order 5400.5, Change 2. Washington, DC.

U.S. Department of Energy, Nevada Operations Office. 1996. Industrial Sites Quality Assurance Project Plan, Nevada Test Site, Nevada, DOE/NV--372, Rev. 1. Las Vegas, NV.

U.S. Ecology and Atlan-Tech, Inc. 1991. Environmental Monitoring Report for the Proposed Ward Valley, California, Low-Level Radioactive Waste (LLRW) Facility, Vols. 1 and 2, Version 2. Roswell, GA: California Department of Health, Environmental Management Branch.

U.S. Environmental Protection Agency. 2004. Region 9 Preliminary Remediation Goals (PRGs). San Francisco, CA.

Yu C., A.J. Zielen, J.J Cheng, D.J. LePoire., E. Gnanapragasam, S. Kamboj, J. Arnish, A. Wallo III, W.A. Williams, and H. Peterson. 2001. User's Manual for RESRAD Version 6, ANL/EAD-4, Argonne National Laboratory, Environmental Assessment Division, Argonne, IL. 


\section{Appendix A}

Corrective Action Investigation Report for CAU 476:

\section{Area 12 T-Tunnel Muckpile, Nevada Test Site}




\section{Table of Contents}

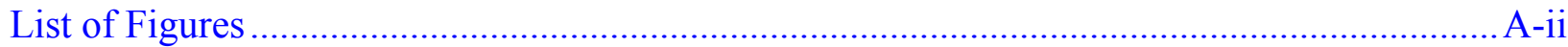

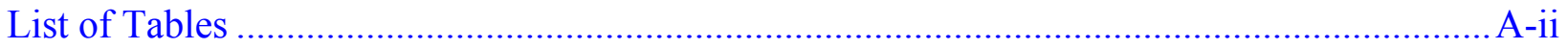

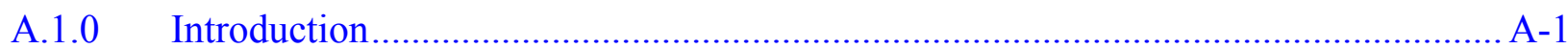

A.1.1 Project Objective ............................................................................. A-1

A.1.2 Report Content .................................................................................. A-3

A.2.0 Field Investigation and Sampling Activities....................................................... A-4

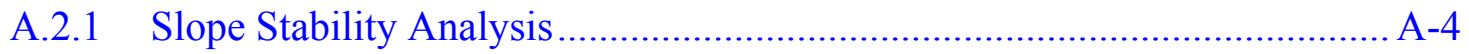

A.2.2 Surface Geophysical Surveys …………............................................. A-4

A.2.3 Borehole Locations ............................................................................ A-5

A.2.4 Subsurface Characterization ……………………................................. A-6

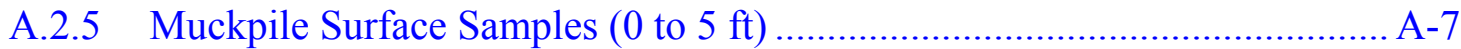

A.2.6 Background Native Soil Samples $(0.5 \mathrm{ft})$.............................................. A-8

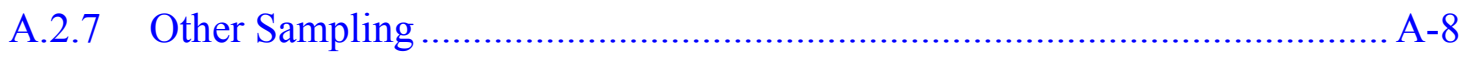

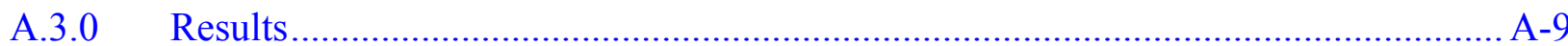

A.3.1 Total VOC and SVOCs..................................................................... A-14

A.3.2 Total Petroleum Hydrocarbons …………............................................ A-14

A.3.3 Total RCRA Metals Results............................................................... A-14

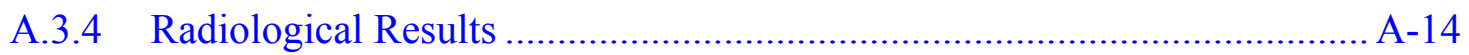

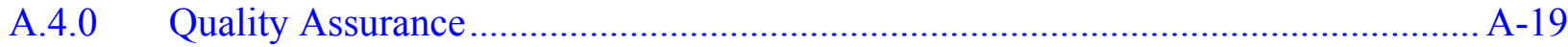

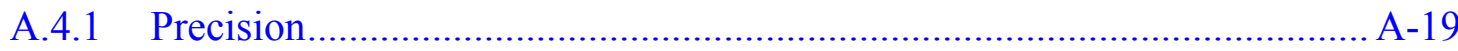

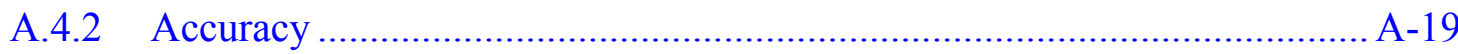

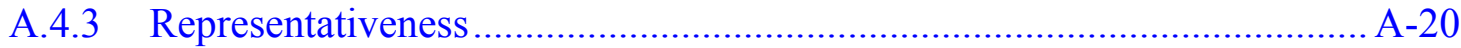

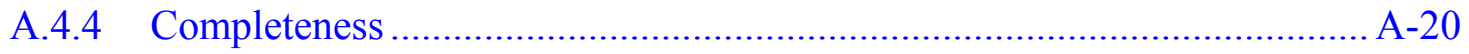

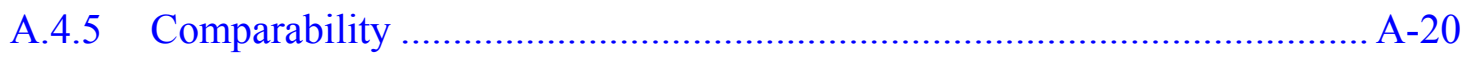

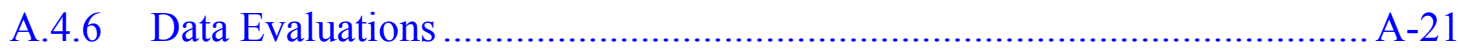

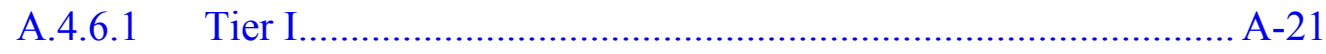

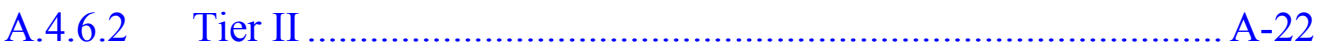

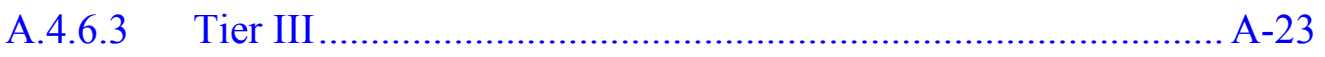

A.4.7 Quality Control Samples....................................................................... A-23

A.4.7.1 Field Quality Control Samples.................................................. A-23

A.4.7.2 Laboratory Quality Control Samples ....................................... A-25

A.4.8 Nonconformances and Field Deficiencies ................................................ A-25

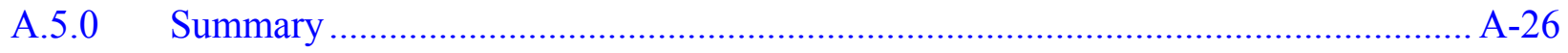

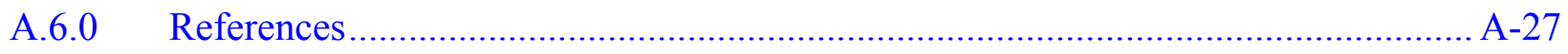




\section{List of Figures}

Number

Title

Page

A.1-1 T-Tunnel Muckpile Sample Location Map

A-2

\section{List of Tables}

Number

Title

Page

A.2-1 Borehole Locations, Total Depth, and Sampling Depths

A-5

A.3-1 Samples Collected and Submitted for Laboratory Analyses for the

CAU 476 Area 12 T-Tunnel Muckpile Corrective Action Investigation ...

A.3-2 Chemical Analytical Methods Used for T-Tunnel Investigation Samples

A.3-3 VOCs, SVOCs, Total Metals, and TCLP Metals Detects for the T-Tunnel Muckpile Investigation

A.3-4 Radionuclide Detects for the T-Tunnel Muckpile Investigation A-18 


\section{A.1.0 Introduction}

This report presents a summary of the field activities and the data collected during the CAI of the T-Tunnel Muckpile. The CAI was controlled and guided by the Corrective Action Investigation Plan for Corrective Action Unit 476: Area 12 T-Tunnel Muckpile, Nevada Test Site, Rev. 0 (DTRA, 2000a). The T-Tunnel Muckpile is identified in the FFACO as CAU 476, CAS 12-06-02 (FFACO, 1996).

The T-Tunnel Muckpile is located approximately 40 miles north of Mercury in Area 12 of the NTS. The T-Tunnel was mined into the bedded ash-flow tuff of Rainier Mesa starting in 1968. Nuclear and nonnuclear testing and mineback operations continued until 1997. The muckpile is estimated to contain approximately 500,000 cubic yards of mining and re-entry debris. Less than 1 percent of this material is considered to be re-entry debris. Additional information relating to the site history, planning, and scope of the investigation is presented in the CAIP (DTRA, 2000a).

\section{A.1.1 Project Objective}

The primary objective of the T-Tunnel CAI was to determine whether the T-Tunnel Muckpile or the underlying native soils have been impacted by COPCs at concentrations that exceed regulatory limits. The data collected during the field effort will enable DTRA to make informed decisions about the future operation, use, or closure of the muckpile site. The following tasks were performed to meet the project objective:

- A sonic drill rig was used to drill 20 holes through the muckpile and $5 \mathrm{ft}$ into the underlying native material (only $2 \mathrm{ft}$, if the native material was bedrock). Seven boreholes to $5 \mathrm{ft}$ were drilled and one location excavated with hand tools to characterize the muckpile surface (Figure A.1-1).

- Continuous cores were collected from all of the boreholes.

- All of the cores were field screened for VOCs and radioactivity for health and safety purposes and to identify depths where optional environmental samples should be collected. 




Figure A.1-1

T-Tunnel Muckpile Sample Location Map 
- Soil samples were collected from random depths within the muckpile, from $5 \mathrm{ft}$ (or $2 \mathrm{ft}$, if bedrock) below the muckpile/native material interface, and from the surface/near-surface (less than $5 \mathrm{ft}$ below ground surface [bgs]) of the muckpile

- Samples were sent to an off-site laboratory for analysis for VOCs, SVOCs, total RCRA metals, and radionuclides.

- Three background samples of native soil were collected using hand tools and analyzed for total RCRA metals and radionuclides at the off-site laboratory.

- All of the cores were described to assess soil and waste physical characteristics.

\section{A.1.2 Report Content}

The CAI report is intended to provide information and data in sufficient detail to support the selection of a preferred corrective action alternative reported in the CADD. The contents of this CAI report are as follows:

- Section A.1.0 of this report is an introduction that includes a description of the objective and scope of the project.

- Section A.2.0 details the muckpile investigation and provides a description of the sample collection activities and locations.

- Section A.3.0 is a summary of the sample analytical results.

- Section A.4.0 discusses the quality assurance (QA) and quality control (QC) procedures that were followed and the results of the QA and QC activities.

- Section A.5.0 summarizes the significant results of the CAI.

- Section A.6.0 lists the references.

To provide a concise summary, the complete field documentation and laboratory data is not contained in this report. These documents are retained in project files. 


\section{A.2.0 Field Investigation and Sampling Activities}

The field investigation and sampling program were managed in accordance with the requirements set forth in the CAIP (DTRA, 2000a). The field activities were performed in accordance with an approved site-specific health and safety plan (ITLV, 2000). The samples were collected and documented by following approved sampling, decontamination, chain-of-custody, shipping, and radiation screening protocols and documentation procedures. Quality control samples (e.g., equipment rinsate blanks, trip blanks, and sample duplicates) were collected as specified in the CAIP and the Industrial Sites Quality Assurance Project Plan (QAPP) (DOE/NV, 1996), and in accordance with approved procedures.

\section{A.2.1 Slope Stability Analysis}

Given the site conditions and proposed operating parameters, a slope stability analysis was not prepared for T-Tunnel. Because of the similarity in construction between the previously investigated N-Tunnel Muckpile and the T-Tunnel Muckpile, the work restrictions that were applied for the N-Tunnel Muckpile drilling were used for the T-Tunnel Muckpile drilling. Conservative assumptions were used in the preparation of the N-Tunnel Muckpile slope stability calculations. Those restrictions included that drilling could be safely conducted at least $50 \mathrm{ft}$ from the edge of the lower bench and $25 \mathrm{ft}$ from the edge of the upper bench. The T-Tunnel Muckpile is smaller in area and not as high from toe to crest as the N-Tunnel Muckpile, so adopting the N-Tunnel Muckpile slope stability restrictions demonstrated a very conservative approach to safely sampling the muckpile.

\section{A.2.2 Surface Geophysical Surveys}

Prior to identifying borehole locations, surface geophysical surveys were conducted to locate approximately 75 full acetylene cylinders that were reported to have been buried in the upper bench of the muckpile. The geophysical surveys were conducted over seven days from January 17 to January 24, 2000.

Electromagnetic (EM) and ground penetrating radar (GPR) methods were used to survey the upper bench of the muckpile. First, EM 31 and an EM 61 surveys were conducted. Then, GPR surveys were conducted over anomalies identified by the EM surveys. No area was identified that positively identified the location of the cylinders; however, the geophysical data were used 
to eliminate areas from consideration as drill targets because of the possibility of drilling into a buried cylinder.

\section{A.2.3 Borehole Locations}

The borehole locations were identified by coordinates which were randomly assigned using an unaligned grid sampling method (Gilbert, 1987). The locations were limited to those areas considered safe for drilling based on the results of the surface geophysical surveys. Prior to the commencement of the drilling operations, Bechtel Nevada $(\mathrm{BN})$ surveyors located the boreholes on the muckpile at the calculated coordinates. Locations for 80 boreholes were staked by the BN surveyors. This included 20 primary holes and three alternate locations for every primary location. The alternate locations were surveyed to allow for uninterrupted field work in the event a primary location could not be sampled.

In addition to the deep holes, eight shallow hole locations were identified based on elevated readings as measured using a field instrument for the detection of low energy radiation (FIDLER) or in areas of surface staining. After completion of the drilling, the exact locations of the shallow soil borings were surveyed using a Trimble global positioning system. A list of the boreholes drilled and the sample depths is provided in Table A.2-1. Figure A.1-1 shows the locations of the drilled boreholes.

Table A.2-1

Borehole Locations, Total Depth, and Sampling Depths

(Page 1 of 2)

\begin{tabular}{|c|c|c|c|c|c|c||}
\hline Hole \# & Northing & Easting & $\begin{array}{c}\text { Collar } \\
\text { Elevation (ft) }\end{array}$ & $\begin{array}{c}\text { Sample } \\
\text { Depth(s) (ft) }\end{array}$ & $\begin{array}{c}\text { Bottom of } \\
\text { Muckpile (ft) }\end{array}$ & $\begin{array}{c}\text { Total Depth } \\
\text { (ft)/Comments }\end{array}$ \\
\hline \hline 2 & 898154 & 646499 & 5,583 & $8.5,56.5$ & 52 & 60 \\
\hline 3 & 898101 & 646485 & 5,582 & $9,61.5$ & 57 & 68 \\
\hline 4 & 898051 & 646479 & 5,581 & $36.5,63.5$ & 59 & 64 \\
\hline $4 \mathrm{~A}$ & 898074 & 646479 & 5,580 & $58,68.5$ & 64 & $\begin{array}{c}72 / \text { alternate } \\
\text { for hole \#1 }\end{array}$ \\
\hline 5 & 898001 & 646477 & 5,581 & $24,51.5$ & 47 & 52 \\
\hline 6 & 897839 & 646329 & 5,596 & $12,47.5$ & 44 & 48 \\
\hline 7 & 897804 & 646312 & 5,596 & $13,40.5$ & 36 & 47 \\
\hline 8 & 898191 & 646455 & 5,582 & $21.5,37.5$ & 33 & 39 \\
\hline 9 & 898106 & 646433 & 5,582 & $31.5,34.5,45.5$ & 41 & 46 \\
\hline
\end{tabular}


Table A.2-1

\section{Borehole Locations, Total Depth, and Sampling Depths}

(Page 2 of 2)

\begin{tabular}{|c|c|c|c|c|c|c|}
\hline Hole \# & Northing & Easting & $\begin{array}{c}\text { Collar } \\
\text { Elevation (ft) }\end{array}$ & $\begin{array}{c}\text { Sample } \\
\operatorname{Depth}(\mathrm{s})(\mathrm{ft})\end{array}$ & $\begin{array}{c}\text { Bottom of } \\
\text { Muckpile (ft) }\end{array}$ & $\begin{array}{l}\text { Total Depth } \\
\text { (ft)/Comments }\end{array}$ \\
\hline 10 & 898021 & 646390 & 5,582 & $11.5,49.5$ & 45 & 51 \\
\hline 11 & 897965 & 646466 & 5,580 & $31.5,39.5,51.5$ & 47 & 52 \\
\hline 12 & 897708 & 646256 & 5,596 & $14,24.5$ & 23 & 26 \\
\hline 13 & 897899 & 646143 & 5,598 & $14,40.5$ & 36 & 42 \\
\hline 14 & 897796 & 646051 & 5,599 & $7.5,23.5$ & 21 & 24 \\
\hline 15 & 897831 & 646091 & 5,598 & $11,28.5$ & 24 & 29 \\
\hline 16 & 897988 & 645854 & 5,600 & $11,16.5$ & 12 & 18 \\
\hline 17 & 897849 & 645982 & 5,599 & 9,25 & 20.5 & 27 \\
\hline 18 & 898000 & 645894 & 5,599 & $3,19.5$ & 15 & 21 \\
\hline 19 & 898035 & 646007 & 5,597 & $4,34.5$ & 30 & 35.5 \\
\hline $20 \mathrm{~A}$ & 898031 & 646220 & 5,597 & $19.5,50.5$ & 45.5 & 51 \\
\hline B1 & 898507 & 645736 & 5,791 & 1 & $\mathrm{~N} / \mathrm{A}$ & 1.5 \\
\hline B2 & 897306 & 646482 & 5,614 & 1 & $\mathrm{~N} / \mathrm{A}$ & 1.5 \\
\hline B3 & 897518 & 644916 & 5,720 & 1 & $N / A$ & 1.5 \\
\hline S1 & 897956 & 646323 & 5,582 & $0.5,2.5$ & $\mathrm{~N} / \mathrm{A}$ & 5 \\
\hline S2 & 897951 & 646345 & 5,581 & 0.5 & $\mathrm{~N} / \mathrm{A}$ & 5 \\
\hline S3 & 897952 & 646372 & 5,581 & 1 & $\mathrm{~N} / \mathrm{A}$ & 5 \\
\hline S4 & 897929 & 646426 & 5,580 & 0.5 & $\mathrm{~N} / \mathrm{A}$ & 5 \\
\hline S5 & 897931 & 646470 & 5,580 & 0.5 & $N / A$ & 5 \\
\hline S6 & 897914 & 646527 & 5,573 & 0.5 & $\mathrm{~N} / \mathrm{A}$ & $\begin{array}{c}1 / \text { hole } \\
\text { dug by hand }\end{array}$ \\
\hline S7 & 897948 & 645876 & 5,599 & 1 & $\mathrm{~N} / \mathrm{A}$ & 5 \\
\hline S8 & 897977 & 645942 & 5,599 & 1 & $\mathrm{~N} / \mathrm{A}$ & 5 \\
\hline
\end{tabular}

$\mathrm{ft}=$ Foot

$\mathrm{N} / \mathrm{A}=$ Not applicable

\section{A.2.4 Subsurface Characterization}

The drilling activities at the T-Tunnel Muckpile were conducted over 19 workdays between April 10 and May 2, 2000.

Deep soil sampling consisted of drilling boreholes through the muckpile and into the native material underneath using a rotosonic drill rig. If the native material was alluvial in nature, the 
borehole was advanced $5 \mathrm{ft}$ into the native material. If the native material was bedrock, the borehole was only advanced $2 \mathrm{ft}$ into the native material or until refusal. The 27 boreholes were drilled to depths ranging from 18 to $72 \mathrm{ft}$ for a total of $907.5 \mathrm{ft}$.

Two soil samples were collected from each borehole, one at a randomly selected depth (the $\mathrm{z}$ depth) and one from the bottom of the borehole. Additional samples were collected from sections of core where field screening indicated elevated levels of VOCs or radionuclides. A total of 22 environmental soil samples were collected to characterize the muckpile and 20 environmental soil samples were collected to characterize the underlying native material. All of the soil samples were sent to an off-site laboratory and analyzed for VOCs, SVOCs, TPH-DRO, total RCRA metals, and radionuclides. An additional aliquot of each sample was sent to the laboratory to archive for potential analysis for Sr-90 and isotopic plutonium for waste management determinations.

\section{A.2.5 Muckpile Surface Samples (0 to $5 \mathrm{ft}$ )}

Shallow soil sampling consisted of drilling boreholes $5 \mathrm{ft}$ deep into the muckpile using the rotosonic drill rig and collecting a soil sample. A total of $35 \mathrm{ft}$ of drilling was completed in seven boreholes to characterize the muckpile surface. An additional sample, S8 (see Figure A.1-1), was collected near the edge of the muckpile using hand tools. Six of the seven shallow boreholes and the location sampled with hand tools were selected based on anomalous radiation readings as measured with the FIDLER. One borehole, S8 (see Figure A.1-1), was selected based on oil staining of the surface material.

One soil sample was collected from each borehole. The sample was collected from the section of core with the highest field-screening readings or, if there were no elevated field-screening readings, the sample was collected from 1 to $2 \mathrm{ft}$ below the muckpile surface. Two samples were collected from boring S1; soils at $0.5 \mathrm{ft}$ exceeded VOC field-screening levels, and soils at $2.5 \mathrm{ft}$ exceeded radionuclide field-screening levels. A total of nine environmental soil samples were collected to characterize the muckpile surface. All of the soil samples were sent to an off-site laboratory to be analyzed for VOCs, SVOCs, TPH-DRO, total RCRA metals, and radionuclides. An additional aliquot of each sample was sent to the laboratory to archive for potential analysis for Sr-90 and isotopic plutonium for waste management determinations. 


\section{A.2.6 Background Native Soil Samples (0.5 ft)}

Background soil samples were collected at three locations near the T-Tunnel Muckpile. The samples were collected from a depth of 6 inches using decontaminated hand tools and disposable Teflon ${ }^{\mathrm{TM}}$ scoops. The samples were sent to an off-site laboratory to be analyzed for total RCRA metals and radionuclides.

\section{A.2.7 Other Sampling}

In addition to the environmental samples, three blind duplicate samples were collected and analyzed to check on the laboratory's precision; three matrix spike (MS)/matrix spike duplicate (MSD) samples were collected to check for matrix interference; two rinsate samples were collected to check on the effectiveness of the decontamination procedures; three field blanks were collected to check on possible environmental interferences; and 19 trip blanks were sent for VOC analysis.

Also, at the start of the field work, a sample on the decontamination water was collected from the water truck and archived. Since no issues arose concerning the possibility that the decontamination water was contaminated, this sample was not analyzed. 


\section{A.3.0 Results}

The analytical results of samples collected from the T-Tunnel Muckpile CAI have been compiled and evaluated to determine the presence and extent of the contamination. The results are summarized in the following subsections. Complete laboratory results are available in the project files.

A total of 54 soil samples and 24 water samples were collected and submitted for analysis. Three of the soil samples were submitted to establish background levels. A list of sample numbers and their relationship to the boreholes is presented in Table A.3-1. The analytical parameters and methods requested for the CAI samples submitted to the off-site laboratory are presented in Table A.3-2. All samples were submitted to Paragon Analytics of Fort Collins, Colorado. Third party data validation was completed by TechLaw, Inc. in Lakewood, Colorado.

Table A.3-1

Samples Collected and Submitted for Laboratory Analyses for the CAU 476 Area 12 T-Tunnel Muckpile Corrective Action Investigation (Page 1 of 4)

\begin{tabular}{|c|c|c|c|c|c|c|}
\hline $\begin{array}{c}\text { Borehole } \\
\text { Number }\end{array}$ & $\begin{array}{l}\text { Sample } \\
\text { Number }\end{array}$ & $\begin{array}{l}\text { Depth } \\
\text { (ft bgs) }\end{array}$ & $\begin{array}{l}\text { Sample } \\
\text { Matrix }\end{array}$ & Soil Type & Comments & $\begin{array}{c}\text { Parameters } \\
\text { Analyzed }\end{array}$ \\
\hline \multirow[t]{2}{*}{2} & TS-02-8.5 & $8-9$ & Soil & Muckpile & - & $\begin{array}{l}\text { Full Suite, } \\
\text { Pu, Sr-90 }\end{array}$ \\
\hline & TS-02-56.5 & $56-57$ & Soil & Native & MS/MSD & Full Suite \\
\hline \multirow{2}{*}{3} & TS-03-09 & $8.5-9.5$ & Soil & Muckpile & - & Full Suite \\
\hline & TS-03-61.5 & $61-62$ & Soil & Native & - & Full Suite \\
\hline \multirow{2}{*}{4} & TS-04-36.5 & $36-37$ & Soil & Muckpile & - & Full Suite \\
\hline & TS-04-63.5 & $63-64$ & Soil & Native & - & Full Suite \\
\hline \multirow{2}{*}{$4 \mathrm{~A}$} & TS-04A-58 & $57.5-58.5$ & Soil & Muckpile & - & $\begin{array}{l}\text { Full Suite, } \\
\text { Pu, Sr-90 }\end{array}$ \\
\hline & TS-04A-68.5 & $68-69$ & Soil & Native & - & $\begin{array}{l}\text { Full Suite, } \\
\text { Pu, Sr-90 }\end{array}$ \\
\hline \multirow{3}{*}{5} & TS-05-24 & $23.5-24.5$ & Soil & Muckpile & - & Full Suite \\
\hline & QTS-05-24 & $23.5-24.5$ & Soil & Muckpile & $\begin{array}{c}\text { Duplicate of } \\
\text { TS-05-24 }\end{array}$ & Full Suite \\
\hline & TS-05-51.5 & $51-52$ & Soil & Native & MS/MSD & Full Suite \\
\hline \multirow{3}{*}{6} & TS-06-12 & $11.5-12.5$ & Soil & Muckpile & - & $\begin{array}{l}\text { Full Suite, } \\
\text { Pu, Sr-90 }\end{array}$ \\
\hline & QTS-06-12 & $11.5-12.5$ & Soil & Muckpile & $\begin{array}{c}\text { Duplicate of } \\
\text { TS-06-12 }\end{array}$ & Full Suite \\
\hline & TS-06-47.5 & $47-48$ & Soil & Native & - & Full Suite \\
\hline
\end{tabular}


Table A.3-1

Samples Collected and Submitted for Laboratory Analyses for the Area 12 CAU 476 T-Tunnel Muckpile Corrective Action Investigation (Page 2 of 4 )

\begin{tabular}{|c|c|c|c|c|c|c|}
\hline $\begin{array}{l}\text { Borehole } \\
\text { Number }\end{array}$ & $\begin{array}{l}\text { Sample } \\
\text { Number }\end{array}$ & $\begin{array}{c}\text { Depth } \\
\text { (ft bgs) }\end{array}$ & $\begin{array}{l}\text { Sample } \\
\text { Matrix }\end{array}$ & Soil Type & Comments & $\begin{array}{l}\text { Parameters } \\
\text { Analyzed }\end{array}$ \\
\hline \multirow{2}{*}{7} & TS-07-13 & $12.5-13.5$ & Soil & Muckpile & - & Full Suite \\
\hline & TS-07-40.5 & $40-41$ & Soil & Muckpile & - & Full Suite \\
\hline \multirow[b]{2}{*}{8} & TS-08-22 & $21-22$ & Soil & Muckpile & - & Full Suite \\
\hline & TS-08-38 & $37-38$ & Soil & Native & - & $\begin{array}{l}\text { Full Suite, } \\
\text { Pu, Sr-90 }\end{array}$ \\
\hline \multirow{3}{*}{9} & TS-09-30 & $30-33$ & Soil & Muckpile & $\begin{array}{c}\text { Exceeded } \\
\text { field screening } \\
\text { level for VOCs }\end{array}$ & Full Suite \\
\hline & TS-09-35 & $34-35$ & Soil & Muckpile & - & Full Suite \\
\hline & TS-09-46 & $45-46$ & Soil & Native & - & Full Suite \\
\hline \multirow[b]{2}{*}{10} & TS-10-12 & $11-12$ & Soil & Muckpile & - & Full Suite \\
\hline & TS-10-50 & $49-50$ & Soil & Native & - & $\begin{array}{l}\text { Full Suite, } \\
\text { Pu, Sr-90 }\end{array}$ \\
\hline \multirow{3}{*}{11} & TS-11-31.5 & $31-32$ & Soil & Muckpile & $\begin{array}{c}\text { Exceeded } \\
\text { field screening } \\
\text { level for VOCs }\end{array}$ & $\begin{array}{l}\text { Full Suite, } \\
\text { Pu, Sr-90 }\end{array}$ \\
\hline & TS-11-39.5 & $39-40$ & Soil & Muckpile & - & Full Suite \\
\hline & TS-11-51.5 & $51-52$ & Soil & Native & - & Full Suite \\
\hline \multirow[b]{2}{*}{12} & TS-12-14 & $13.5-14.5$ & Soil & Muckpile & - & Full Suite \\
\hline & TS-12-24.5 & $24-25$ & Soil & Native & - & $\begin{array}{l}\text { Full Suite, } \\
\text { Pu, Sr-90 }\end{array}$ \\
\hline \multirow{2}{*}{13} & TS-13-14 & $13.5-14.5$ & Soil & Muckpile & - & Full Suite \\
\hline & TS-13-41 & $40-41$ & Soil & Native & - & Full Suite \\
\hline \multirow{2}{*}{14} & TS-14-08 & $7-8$ & Soil & Muckpile & - & Full Suite \\
\hline & TS-14-24 & $23-24$ & Soil & Native & - & Full Suite \\
\hline \multirow{2}{*}{15} & TS-15-11.5 & $10.5-11.5$ & Soil & Muckpile & - & Full Suite \\
\hline & TS-15-29 & $28-29$ & Soil & Native & - & Full Suite \\
\hline \multirow{2}{*}{16} & TS-16-11 & $10.5-11.5$ & Soil & Muckpile & - & Full Suite \\
\hline & TS-16-17 & $16-17$ & Soil & Native & - & Full Suite \\
\hline \multirow{2}{*}{17} & TS-17-9.5 & $8.5-9.5$ & Soil & Muckpile & - & Full Suite \\
\hline & TS-17-25 & $24.5-25.5$ & Soil & Native & MS/MSD & Full Suite \\
\hline \multirow{2}{*}{18} & TS-18-3.0 & $2.5-3.5$ & Soil & Muckpile & - & Full Suite \\
\hline & TS-18-20 & $19-20$ & Soil & Native & - & Full Suite \\
\hline
\end{tabular}


Table A.3-1

Samples Collected and Submitted for Laboratory Analyses for the Area 12 CAU 476 T-Tunnel Muckpile Corrective Action Investigation (Page 3 of 4)

\begin{tabular}{|c|c|c|c|c|c|c|}
\hline $\begin{array}{l}\text { Borehole } \\
\text { Number }\end{array}$ & $\begin{array}{l}\text { Sample } \\
\text { Number }\end{array}$ & $\begin{array}{l}\text { Depth } \\
\text { (ft bgs) }\end{array}$ & $\begin{array}{l}\text { Sample } \\
\text { Matrix }\end{array}$ & Soil Type & Comments & $\begin{array}{l}\text { Parameters } \\
\text { Analyzed }\end{array}$ \\
\hline \multirow[t]{2}{*}{19} & TS-19-4.0 & $3.5-4.5$ & Soil & Muckpile & - & $\begin{array}{l}\text { Full Suite, } \\
\mathrm{Pu}, \text { Sr-90 }\end{array}$ \\
\hline & TS-19-35 & $34-35$ & Soil & Native & - & Full Suite \\
\hline \multirow{3}{*}{$20 a$} & TS-20-20 & $19-20$ & Soil & Muckpile & - & Full Suite \\
\hline & TS-20-51 & $50-51$ & Soil & Native & - & Full Suite \\
\hline & QTS-20-51 & $50-51$ & Soil & Native & $\begin{array}{l}\text { Duplicate of } \\
\text { TS-20-51 }\end{array}$ & Full Suite \\
\hline \multirow{2}{*}{ S1 } & TS-S1-0.5 & $0-1$ & Soil & Muckpile & $\begin{array}{l}\text { Exceeded } \\
\text { field screening } \\
\text { level for VOCs }\end{array}$ & $\begin{array}{l}\text { Full Suite, } \\
\text { Pu, Sr-90 }\end{array}$ \\
\hline & TS-S1-2.5 & $2-3$ & Soil & Muckpile & $\begin{array}{l}\text { Exceeded } \\
\text { field screening } \\
\text { level for alpha }\end{array}$ & Full Suite \\
\hline S2 & TS-S2-0.5 & $0-1$ & Soil & Muckpile & - & $\begin{array}{l}\text { Full Suite, } \\
\text { Pu, Sr-90 }\end{array}$ \\
\hline S3 & TS-S3-01 & $0.5-1.5$ & Soil & Muckpile & - & Full Suite \\
\hline S4 & TS-S4-0.5 & $0-1$ & Soil & Muckpile & - & $\begin{array}{l}\text { Full Suite, } \\
\text { Pu, Sr-90 }\end{array}$ \\
\hline S5 & TS-S5-0.5 & $0-1$ & Soil & Muckpile & - & $\begin{array}{l}\text { Full Suite, } \\
\text { Pu, Sr-90 }\end{array}$ \\
\hline S6 & TS-S6-0.5 & $0-1$ & Soil & Muckpile & - & $\begin{array}{l}\text { Full Suite, } \\
\text { Pu, Sr-90 }\end{array}$ \\
\hline S7 & TS-S7-01 & $0.5-1.5$ & Soil & Muckpile & - & Full Suite \\
\hline S8 & TS-S8-01 & $0.5-1.5$ & Soil & Muckpile & - & Full Suite \\
\hline B1 & TS-B1-01 & $0.5-1.5$ & Soil & Native & $\begin{array}{l}\text { Background } \\
\text { Sample }\end{array}$ & $\begin{array}{c}\text { RCRA } \\
\text { Metals, } \\
\text { Gamma } \\
\text { Spec, Pu, } \\
\text { Sr-90 }\end{array}$ \\
\hline B2 & TS-B2-01 & $0.5-1.5$ & Soil & Native & $\begin{array}{l}\text { Background } \\
\text { Sample }\end{array}$ & $\begin{array}{c}\text { RCRA } \\
\text { Metals, } \\
\text { Gamma } \\
\text { Spec, Pu, } \\
\text { Sr-90 }\end{array}$ \\
\hline B3 & TS-B3-01 & $0.5-1.5$ & Soil & Native & $\begin{array}{l}\text { Background } \\
\text { Sample }\end{array}$ & $\begin{array}{c}\text { RCRA } \\
\text { Metals, } \\
\text { Gamma } \\
\text { Spec, Pu, } \\
\text { Sr-90 }\end{array}$ \\
\hline $\mathrm{N} / \mathrm{A}$ & TW-01 & $\mathrm{N} / \mathrm{A}$ & Water & $N / A$ & Source Blank & Discarded \\
\hline
\end{tabular}


Table A.3-1

Samples Collected and Submitted for Laboratory Analyses for the Area 12 CAU 476 T-Tunnel Muckpile Corrective Action Investigation (Page 4 of 4 )

\begin{tabular}{|c|c|c|c|c|c|c|}
\hline $\begin{array}{l}\text { Borehole } \\
\text { Number }\end{array}$ & $\begin{array}{l}\text { Sample } \\
\text { Number }\end{array}$ & $\begin{array}{l}\text { Depth } \\
\text { (ft bgs) }\end{array}$ & $\begin{array}{c}\text { Sample } \\
\text { Matrix }\end{array}$ & Soil Type & Comments & $\begin{array}{c}\text { Parameters } \\
\text { Analyzed }\end{array}$ \\
\hline $\mathrm{N} / \mathrm{A}$ & TW-02 & N/A & Water & $N / A$ & $\begin{array}{l}\text { Equipment } \\
\text { Rinse Blank }\end{array}$ & Full Suite \\
\hline $\mathrm{N} / \mathrm{A}$ & TW-03 & N/A & Water & $\mathrm{N} / \mathrm{A}$ & Field Blank & Full Suite \\
\hline $\mathrm{N} / \mathrm{A}$ & TW-04 & N/A & Water & $N / A$ & $\begin{array}{l}\text { Equipment } \\
\text { Rinse Blank }\end{array}$ & Full Suite \\
\hline$N / A$ & TW-05 & N/A & Water & $N / A$ & Field Blank & Full Suite \\
\hline N/A & TW-06 & N/A & Water & N/A & Field Blank & Full Suite \\
\hline $\mathrm{N} / \mathrm{A}$ & TR-01 & $N / A$ & Water & $\mathrm{N} / \mathrm{A}$ & Trip Blank & VOC \\
\hline N/A & TR-02 & N/A & Water & N/A & Trip Blank & VOC \\
\hline N/A & TR-03 & N/A & Water & N/A & Trip Blank & VOC \\
\hline $\mathrm{N} / \mathrm{A}$ & TR-04 & N/A & Water & $\mathrm{N} / \mathrm{A}$ & Trip Blank & VOC \\
\hline $\mathrm{N} / \mathrm{A}$ & TR-05 & N/A & Water & $\mathrm{N} / \mathrm{A}$ & Trip Blank & VOC \\
\hline N/A & TR-06 & $N / A$ & Water & $\mathrm{N} / \mathrm{A}$ & Trip Blank & VOC \\
\hline N/A & TR-07 & N/A & Water & N/A & Trip Blank & VOC \\
\hline N/A & TR-08 & N/A & Water & N/A & Trip Blank & VOC \\
\hline N/A & TR-09 & N/A & Water & N/A & Trip Blank & VOC \\
\hline$N / A$ & TR-10 & $N / A$ & Water & $N / A$ & Trip Blank & VOC \\
\hline N/A & TR-11 & N/A & Water & $\mathrm{N} / \mathrm{A}$ & Trip Blank & VOC \\
\hline N/A & TR-12 & N/A & Water & N/A & Trip Blank & VOC \\
\hline$N / A$ & TR-13 & $N / A$ & Water & $N / A$ & Trip Blank & VOC \\
\hline$N / A$ & TR-14 & $\mathrm{N} / \mathrm{A}$ & Water & N/A & Trip Blank & VOC \\
\hline N/A & TR-15 & N/A & Water & $\mathrm{N} / \mathrm{A}$ & Trip Blank & VOC \\
\hline $\mathrm{N} / \mathrm{A}$ & TR-16 & $N / A$ & Water & $\mathrm{N} / \mathrm{A}$ & Trip Blank & VOC \\
\hline N/A & TR-17 & N/A & Water & N/A & Trip Blank & VOC \\
\hline N/A & TR-18 & N/A & Water & N/A & Trip Blank & VOC \\
\hline$N / A$ & TR-19 & $N / A$ & Water & $N / A$ & Trip Blank & VOC \\
\hline
\end{tabular}

Full Suite = VOC, SVOC, TPH-DRO/oil, RCRA Metals, Gamma Spectroscopy

$\mathrm{DRO}=$ Diesel-range organics $\mathrm{ft}$ bgs $=$ Feet below ground surface $\mathrm{N} / \mathrm{A}=$ Not applicable MS = Matrix spike

MSD = Matrix spike duplicate

$\mathrm{Pu}=$ Plutonium
RCRA = Resource Conservation and Recovery Act $\mathrm{Sr}=$ Strontium SVOC = Semivolatile organic compound $\mathrm{TPH}=$ Total petroleum hydrocarbons VOC $=$ Volatile organic compound 
Table A.3-2

Chemical Analytical Methods Used for T-Tunnel Investigation Samples

\begin{tabular}{|c|c|c|}
\hline Analyte & Medium $^{a}$ & Analytical Method \\
\hline \multirow{2}{*}{ Total VOCs } & Water & \multirow{2}{*}{$8260 B^{b}$} \\
\hline & Soil & \\
\hline \multirow{2}{*}{ Total SVOCs } & Water & \multirow{2}{*}{$8270 C^{b}$} \\
\hline & Soil & \\
\hline $\begin{array}{l}\text { Total RCRA Metals } \\
\text { Arsenic } \\
\text { Barium } \\
\text { Cadmium } \\
\text { Chromium } \\
\text { Lead } \\
\text { Selenium } \\
\text { Silver } \\
\text { Mercury }\end{array}$ & Water & $6010 \mathrm{~B} / 7470 \mathrm{~A}^{\mathrm{b}}$ \\
\hline $\begin{array}{l}\text { Arsenic } \\
\text { Barium } \\
\text { Cadmium } \\
\text { Chromium } \\
\text { Lead } \\
\text { Selenium } \\
\text { Silver } \\
\text { Mercury }\end{array}$ & Soil & $6010 \mathrm{~B} / 7471 \mathrm{~A}^{\mathrm{b}}$ \\
\hline \multirow{2}{*}{ Total Petroleum Hydrocarbons } & $\begin{array}{c}\text { Water } \\
\text { (diesel/oil) }\end{array}$ & \multirow{2}{*}{ 8015B Modified ${ }^{b}$} \\
\hline & $\begin{array}{c}\text { Soil } \\
\text { (diesel/oil) }\end{array}$ & \\
\hline \multirow{2}{*}{ Gamma Spectroscopy } & Water & EPA $901.1^{\mathrm{c}, \mathrm{d}}$ \\
\hline & Soil & HASL $300^{\mathrm{c}, \mathrm{e}}$ \\
\hline \multirow{2}{*}{ Isotopic Plutonium } & Water & \multirow{2}{*}{ HASL $300^{\mathrm{c}, \mathrm{e}}$} \\
\hline & Soil & \\
\hline \multirow{2}{*}{ Strontium-90 } & Water & \multirow{2}{*}{ ASTM D 5811-95 } \\
\hline & Soil & \\
\hline
\end{tabular}

${ }^{a}$ Includes methods for quality control (water) samples.

${ }^{\mathrm{b}}$ Test Methods for Evaluating Solid Waste Physical/Chemical Methods, 3rd Edition, Parts 1-4, SW-846 CD ROM.

Washington, DC (EPA, 1996).

${ }^{\circ}$ Or equivalent method

${ }^{\mathrm{d}}$ Prescribed Procedures for Measurement of Radioactivity in Drinking Water (EPA, 1980)

environmental Measurements Laboratory Procedure Manual, HASL-300, 28th Edition, February (DOE, 1997)

'Standard Test Method for Strontium-90 in Water (ASTM, 1995)

ASTM = American Society for Testing and Materials

$\mathrm{EPA}=$ U.S. Environmental Protection Agency

$\mathrm{HASL}=$ Health and Safety Laboratory
RCRA = Resource Conservation and Recovery Act SVOC $=$ Semivolatile organic compound

VOC $=$ Volatile organic compound 


\section{A.3.1 Total VOC and SVOCs}

Volatile organics compounds and SVOCs were detected in samples throughout the muckpile, in the native soils, and in the background samples at levels above the method detection limit. No VOCs or SVOCs were detected above the action levels established in the CAIP (DTRA, 2000a).

\section{A.3.2 Total Petroleum Hydrocarbons}

Of the TPH-DRO detections, there were four that exceeded the action levels. Concentrations detected in the samples ranged from $110 \mathrm{mg} / \mathrm{kg}$ to $1,200 \mathrm{mg} / \mathrm{kg}$. All of the locations were shallow subsurface.

\section{A.3.3 Total RCRA Metals Results}

If the total metals result for a specific metal divided by 20 exceeded the maximum concentration of contamination (MCC) for the toxicity characteristic (40 CFR 261.24 [CFR, 1996]), the sample was reanalyzed using Toxicity Characteristic Leaching Procedure (TCLP) protocols to determine if the contaminant level of the leachate would exceed the MCC. Eight samples were reanalyzed using TCLP protocols, and the results are reported in Table A.3-3.

Arsenic was the only metal detected at levels exceeding the industrial Preliminary Remediation Goal (PRG) $(2.7 \mathrm{mg} / \mathrm{kg})$ in 36 of the 57 soil samples collected, including one of the background samples. However, these concentrations were below the average concentration of $23 \mathrm{mg} / \mathrm{kg}$ for the NTS (Moore, 1999).

\section{A.3.4 Radiological Results}

Analytical results for soils from the muckpile and native soils beneath the muckpile reported in Table A.3-4 indicate naturally occurring isotopes or isotopes in concentrations that were not statistically significant when compared to background. Several isotopes associated with weapons testing were found in the surface soils of the muckpile in concentrations greater than background. These isotopes included Cs-137, Am-241, Pu-238, Pu-239, Sb-125, and Sr-90. 
Table A.3-3

VOCs, SVOCs, Total Metals, and TCLP Metals Detects for the T-Tunnel Muckpile Investigation (Page 1 of 3 )

\begin{tabular}{|c|c|c|c|c|c|c|c|c|c|c|c|c|}
\hline & Units & PAL & $\begin{array}{l}\text { Background } \\
\text { Range }\end{array}$ & $\begin{array}{l}\text { No. of } \\
\text { Detects }\end{array}$ & $\begin{array}{c}\text { Surface } \\
\text { Soil } \\
\text { Range }\end{array}$ & $\begin{array}{l}\text { No. of } \\
\text { Detects }\end{array}$ & $\begin{array}{c}\text { Subsurface } \\
\text { Muckpile } \\
\text { Range }\end{array}$ & $\begin{array}{l}\text { No. of } \\
\text { Detects }\end{array}$ & $\begin{array}{l}\text { Native } \\
\text { Range }\end{array}$ & $\begin{array}{l}\text { No. of } \\
\text { Detects }\end{array}$ & $\begin{array}{l}\text { QA/QC } \\
\text { Range }\end{array}$ & $\begin{array}{l}\text { No. of } \\
\text { Detects }\end{array}$ \\
\hline \multicolumn{13}{|c|}{ Volatile Organic Compounds } \\
\hline 1,1-Dichloroethene & $\mu \mathrm{g} / \mathrm{kg}$ & 410,000 & & & & & $1.1-1.3$ & 2 & $1.1-1.3$ & 3 & & \\
\hline 1,2,3-Trichlorobenzene & $\mu \mathrm{g} / \mathrm{kg}$ & 220,000 & & & & & 1.4 & 1 & & & & \\
\hline 1,2,4-Trichlorobenzene & $\mu \mathrm{g} / \mathrm{kg}$ & 220,000 & & & & & 1.2 & 1 & & & & \\
\hline 1,2,4-Trimethylbenzene & $\mu \mathrm{g} / \mathrm{kg}$ & 170,000 & & & & & 1 & 1 & & & & \\
\hline 4Methyl-2Pentanone & $\mu \mathrm{g} / \mathrm{kg}$ & $47,000,000$ & & & & & 2.7 & 1 & 2 & 1 & & \\
\hline Acetone & $\mu \mathrm{g} / \mathrm{kg}$ & $54,000,000$ & & & 25 & 1 & $9.4-21$ & 8 & $8.6-19$ & 5 & & \\
\hline Acetone & $\mu g / L$ & $54,000,000$ & & & & & & & & & $10-16$ & 5 \\
\hline Bromodichloromethane & $\mu g / L$ & 1,800 & & & & & & & & & $3.3-4.6$ & 5 \\
\hline Chloroethane & $\mu \mathrm{g} / \mathrm{L}$ & 6,500 & & & & & & & & & $0.74-0.79$ & 3 \\
\hline Chloroform & $\mu g / L$ & 470 & & & & & & & & & $4.9-7.6$ & 5 \\
\hline Chloromethane & $\mu g / L$ & 160,000 & & & & & & & & & $2.9-4.1$ & 4 \\
\hline Dibromochloromethane & $\mu g / L$ & 2,600 & & & & & & & & & $0.89-1.2$ & 5 \\
\hline Naphthalene & $\mu \mathrm{g} / \mathrm{kg}$ & 190,000 & & & & & 1.6 & 1 & & & & \\
\hline Naphthalene & $\mu g / L$ & 190,000 & & & & & & & & & $0.67-0.76$ & 3 \\
\hline N-Butylbenzene & $\mu \mathrm{g} / \mathrm{kg}$ & 240,000 & & & & & 0.88 & 1 & & & & \\
\hline Toluene & $\mu \mathrm{g} / \mathrm{kg}$ & 520,000 & & & & & 0.9 & 1 & & & & \\
\hline Trichloroethene & $\mu \mathrm{g} / \mathrm{kg}$ & 110 & & & & & $0.49-0.76$ & 2 & & & & \\
\hline
\end{tabular}


Table A.3-3

VOCs, SVOCs, Total Metals, and TCLP Metals Detects for the T-Tunnel Muckpile Investigation (Page 2 of 3 )

\begin{tabular}{|c|c|c|c|c|c|c|c|c|c|c|c|c|}
\hline & Units & PAL & $\begin{array}{l}\text { Background } \\
\text { Range }\end{array}$ & $\begin{array}{c}\text { No. of } \\
\text { Detects }\end{array}$ & $\begin{array}{l}\text { Surface } \\
\text { Soil } \\
\text { Range }\end{array}$ & $\begin{array}{c}\text { No. of } \\
\text { Detects }\end{array}$ & $\begin{array}{c}\text { Subsurface } \\
\text { Muckpile } \\
\text { Range }\end{array}$ & $\begin{array}{c}\text { No. of } \\
\text { Detects }\end{array}$ & $\begin{array}{l}\text { Native } \\
\text { Range }\end{array}$ & $\begin{array}{c}\text { No. of } \\
\text { Detects }\end{array}$ & $\begin{array}{l}\text { QA/QC } \\
\text { Range }\end{array}$ & $\begin{array}{l}\text { No. of } \\
\text { Detects }\end{array}$ \\
\hline \multicolumn{13}{|c|}{ Semivolatile Compounds } \\
\hline Trichlorotrifluoroethane & $\mu \mathrm{g} / \mathrm{kg}$ & $2,000,000$ & & & & & $1-1.1$ & 2 & $0.8-0.96$ & 2 & & \\
\hline Benzoic Acid & $\mu \mathrm{g} / \mathrm{kg}$ & $100,000,000$ & & & & & & & 270 & 1 & & \\
\hline Bis(2-ethylhexyl)phthalate & $\mu \mathrm{g} / \mathrm{kg}$ & 120,000 & & & & & 350 & 1 & & & & \\
\hline Bis(2-ethylhexyl)phthalate & $\mu g / L$ & 120,000 & & & & & & & & & $19-61$ & 2 \\
\hline Di-n-butylphthalate & $\mu \mathrm{g} / \mathrm{kg}$ & $62,000,000$ & & & 26,000 & 1 & & & & & & \\
\hline \multicolumn{13}{|c|}{ Petroleum Hydrocarbons } \\
\hline Diesel-range organics & $\mathrm{mg} / \mathrm{kg}$ & 100 & & & $25-1,200$ & 5 & $22-330$ & 6 & 47 & 1 & & \\
\hline \multicolumn{13}{|c|}{ Metals (Total RCRA or TCLP) } \\
\hline Arsenic & $\mathrm{mg} / \mathrm{kg}$ & 23 & $2-3.8$ & 3 & $2.2-5.8$ & 9 & $1.6-13$ & 24 & $1.2-7.2$ & 20 & & \\
\hline Arsenic (TCLP) & $\mu g / L$ & 5 & & & $0.037-0.04$ & 2 & $0.072-0.17$ & 2 & & & & \\
\hline Barium (total) & $\mathrm{mg} / \mathrm{kg}$ & 67,000 & $78-110$ & 3 & $44-4,500$ & 9 & $19-3300$ & 24 & $30-200$ & 21 & & \\
\hline Barium (TCLP) & $\mu g / L$ & 100 & & & $0.2-0.27$ & 3 & $0.19-0.23$ & 3 & & & & \\
\hline Cadmium & $\mathrm{mg} / \mathrm{kg}$ & 450 & $0.046-0.088$ & 3 & $0.05-0.24$ & 9 & $0.04-0.4$ & 7 & & & & \\
\hline Chromium (total) & $\mathrm{mg} / \mathrm{kg}$ & 450 & $3.5-6$ & 3 & $0.98-12$ & 9 & $0.42-11$ & 20 & $0.57-10$ & 18 & & \\
\hline Chromium (TCLP) & $\mu g / L$ & 5 & & & $0.028-0.041$ & 4 & & & & & & \\
\hline Chromium (water) & $\mu g / L$ & 29 & & & & & & & & & 0.0048 & 1 \\
\hline
\end{tabular}


Table A.3-3

VOCs, SVOCs, Total Metals, and TCLP Metals Detects for the T-Tunnel Muckpile Investigation

(Page 3 of 3 )

\begin{tabular}{|c|c|c|c|c|c|c|c|c|c|c|c|c|}
\hline & Units & PAL & $\begin{array}{l}\text { Background } \\
\text { Range }\end{array}$ & $\begin{array}{l}\text { No. of } \\
\text { Detects }\end{array}$ & $\begin{array}{c}\text { Surface } \\
\text { Soil } \\
\text { Range }\end{array}$ & $\begin{array}{l}\text { No. of } \\
\text { Detects }\end{array}$ & $\begin{array}{c}\text { Subsurface } \\
\text { Muckpile } \\
\text { Range }\end{array}$ & $\begin{array}{c}\text { No. of } \\
\text { Detects }\end{array}$ & $\begin{array}{l}\text { Native } \\
\text { Range }\end{array}$ & $\begin{array}{l}\text { No. of } \\
\text { Detects }\end{array}$ & $\begin{array}{l}\text { QA/QC } \\
\text { Range }\end{array}$ & $\begin{array}{l}\text { No. of } \\
\text { Detects }\end{array}$ \\
\hline Lead (total) & $\mathrm{mg} / \mathrm{kg}$ & 800 & $9.1-12$ & 3 & $6.2-63$ & 9 & $3-210$ & 24 & $2.7-18$ & 21 & & \\
\hline Lead (TCLP) & $\mathrm{mg} / \mathrm{L}$ & 5 & & & & & $0.29-7.9$ & 4 & & & & \\
\hline Mercury & $\mathrm{mg} / \mathrm{kg}$ & 310 & & & & & 0.079 & 1 & & & & \\
\hline Selenium & $\mathrm{mg} / \mathrm{kg}$ & 5,100 & & & 0.61 & 1 & $0.62-4.1$ & 5 & $0.6-1.5$ & 3 & & \\
\hline Selenium (TCLP) & $\mu \mathrm{g} / \mathrm{L}$ & 1 & & & & & 0.043 & 1 & & & & \\
\hline Silver & $\mathrm{mg} / \mathrm{kg}$ & 5,100 & & & $0.31-0.32$ & 2 & $0.47-2.4$ & 2 & $0.18-0.8$ & 5 & & \\
\hline
\end{tabular}

$\mathrm{mg} / \mathrm{kg}=$ Milligrams per kilogram

$\mathrm{mg} / \mathrm{L}=$ Milligrams per liter

$P A L=$ Preliminary action level

$\mathrm{QA}=$ Quality assurance

RCRA = Resource Conservation and Recovery Act

TCLP = Toxicity Characteristic Leaching Procedure

$\mu \mathrm{g} / \mathrm{kg}=$ Micrograms per kilogram

$\mu \mathrm{g} / \mathrm{L}=$ Micrograms per liter 
Table A.3-4

Radionuclide Detects for the T-Tunnel Muckpile Investigation

\begin{tabular}{|c|c|c|c|c|c|c|c|c|c|c|}
\hline Radionuclide & Units & PAL & $\begin{array}{c}\text { Background } \\
\text { Range }^{\mathrm{a}}\end{array}$ & $\begin{array}{l}\text { No. of } \\
\text { Detects }\end{array}$ & $\begin{array}{c}\text { Surface } \\
\text { Soil Range }\end{array}$ & $\begin{array}{c}\text { No. of } \\
\text { Detects }\end{array}$ & $\begin{array}{c}\text { Subsurface } \\
\text { Muckpile } \\
\text { Range }\end{array}$ & $\begin{array}{l}\text { No. of } \\
\text { Detects }\end{array}$ & $\begin{array}{l}\text { Native } \\
\text { Range }^{b}\end{array}$ & $\begin{array}{c}\text { No. of } \\
\text { Detects }\end{array}$ \\
\hline Actinium-228 & $\mathrm{pCi} / \mathrm{g}$ & 5 & $2.11-3.2$ & 3 & $1.99-3.9$ & 6 & $1.36-3.92$ & 21 & $1.49-5.8$ & 19 \\
\hline Americium-241 & $\mathrm{pCi} / \mathrm{g}$ & 12.7 & 2.8 & 1 & & & & & 0.62 & 1 \\
\hline Bismuth-212 & $\mathrm{pCi} / \mathrm{g}$ & 5 & & & & & 3.2 & 2 & 5.6 & 1 \\
\hline Bismuth-214 & $\mathrm{pCi} / \mathrm{g}$ & 5 & $1.16-2.4$ & 3 & 1.67 & 1 & $0.92-2.09$ & 17 & $1.1-3.22$ & 18 \\
\hline Cobalt-60 & $\mathrm{pCi} / \mathrm{g}$ & 2.7 & & & 1.76 & 1 & & & & \\
\hline Cesium-137 & $\mathrm{pCi} / \mathrm{g}$ & 12.2 & $1.14-13.5$ & 2 & $0.93-382$ & 7 & 0.58 & 1 & & \\
\hline Potassium-40 & $\mathrm{pCi} / \mathrm{g}$ & $\mathrm{N} / \mathrm{A}$ & $30.1-32.2$ & 3 & $23.7-34.6$ & 9 & $25.3-49$ & 24 & $22.2-40.2$ & 21 \\
\hline Lead-212 & $\mathrm{pCi} / \mathrm{g}$ & 5 & $2.12-2.76$ & 3 & $0.96-3.27$ & 8 & $0.88-3.54$ & 24 & $1.29-4.95$ & 21 \\
\hline Lead-214 & $\mathrm{pCi} / \mathrm{g}$ & 5 & $1.27-1.59$ & 3 & $0.82-2.3$ & 4 & $0.9-2.08$ & 17 & $1.13-2.96$ & 21 \\
\hline Plutonium-238 & $\mathrm{pCi} / \mathrm{g}$ & 13 & $0.307-1.03$ & 2 & $0.179-0.91$ & 3 & & & & \\
\hline Plutonium-239 & $\mathrm{pCi} / \mathrm{g}$ & 12.7 & $0.095-9.6$ & 3 & $0.54-2.87$ & 3 & & & & \\
\hline Antimony-125 & $\mathrm{pCi} / \mathrm{g}$ & 18.1 & & & 3 & 1 & & & & \\
\hline Strontium-90 & $\mathrm{pCi} / \mathrm{g}$ & 838 & 1.13 & 1 & $2.27-13$ & 3 & & & & \\
\hline Thallium-208 & $\mathrm{pCi} / \mathrm{g}$ & 5 & $0.7-0.88$ & 3 & $0.41-0.64$ & 4 & $0.6-1.15$ & 22 & $0.64-1.45$ & 20 \\
\hline
\end{tabular}

Background range of isotopes from three undisturbed samples collected during the investigation

Range of isotopes found in native soils beneath the muckpile.

$\mathrm{N} / \mathrm{A}=$ Not applicable

$\mathrm{PAL}=$ Preliminary action level

$\mathrm{pCi} / \mathrm{g}=$ Picocuries per gram 


\section{A.4.0 Quality Assurance}

The following text outlines the results of the QA/QC activities. Detailed information on the QA program for this CAI is contained in the Industrial Sites QAPP (DOE/NV, 1996).

Quality control results are typically discussed in terms of the five PARCC parameters (precision, accuracy, representativeness, completeness, and comparability) as described in the following sections. The data met the QA requirements and are considered acceptable to support the conclusions presented in this $\mathrm{CADD} / \mathrm{CR}$.

\section{A.4.1 Precision}

Precision is a quantitative measure of the variability of a group of measurements from their average value. Precision is assessed for inorganic analyses by collecting and analyzing duplicate field samples and comparing the results with the original sample. Precision is also assessed by creating, preparing, analyzing, and comparing laboratory duplicates from one or more field samples for inorganic analyses. For organic analyses, results are compared to the MS/MSD results. Precision is reported as relative percent difference (RPD) which is calculated as the difference between the measured concentrations of duplicate samples, divided by the average of the two concentrations, and multiplied by 100. Any deviations from these requirements have been documented and explained and the related data qualified accordingly. The qualification process is described in Section A.4.6 and Appendix C of this report.

\section{A.4.2 Accuracy}

Accuracy is defined as the nearness of a measurement to the true or accepted reference value. It is the composite of the random and systematic components of the measurement system and measures bias in a measurement system. The random component of accuracy is measured and documented through the analyses of spiked samples. Sampling accuracy is assessed by evaluating the results of spiked samples and laboratory control samples. Accuracy measurements are calculated as percent recovery by dividing the measured sample concentration by the true concentration and multiplying the quotient by 100 .

Field accuracy is assessed by confirming that the documents of record track the sample from origin, through transfer of custody, to disposal. The goal of field accuracy is for all samples to be collected from the correct locations at the correct time, placed in a correctly labeled container with the correct preservative, and sealed with custody tape to prevent tampering. All samples 
collected for this sampling event were properly collected and forwarded to the laboratory as described above.

\section{A.4.3 Representativeness}

Representativeness expresses the degree to which sample data accurately and precisely represent a characteristic of a population, parameter variations at a sampling point, or an environmental condition (EPA, 1987). Sample representativeness was achieved through the implementation of a sampling program designed to ensure proper sampling locations, number of samples, and the use of validated analytical methods. Representativeness was assessed through analysis of duplicate samples. Representativeness of the samples taken in this sampling event was assured by collecting the required samples shown in Table A.3-1 and by analyzing them using the approved analytical methods shown in Table A.3-2.

\section{A.4.4 Completeness}

Completeness is defined as the percentage of measurements made that are judged to be valid. A sampling and analytical requirement with 90 percent confidence level was established for this project (DTRA, 2000a). The sampling and analytical program were executed in accordance with approved field sampling instructions (DTRA, 2000b). The specified sampling locations were used as planned. All specified samples were collected and all sample containers reached the laboratory intact and properly preserved (when applicable). For all samples, sample temperature was maintained during shipment to the laboratory, and sample chain of custody was maintained during sample storage and/or shipment. A more detailed discussion of the data quality indicators is presented in Appendix C.

\section{A.4.5 Comparability}

Comparability is a qualitative parameter expressing the confidence with which one dataset can be compared to another (EPA, 1987). To ensure comparability, the CAU 476 field sampling activities were performed and documented in accordance with approved procedures; a standardized sampling approach and analytical methodology were used; and all samples were collected per the CAIP (DTRA, 2000a). Approved standardized methods and procedures were also used to analyze and report the data (e.g., EPA SW-846, "Contract Laboratory Program Statement of Work" [CLP] [EPA, 1994b and c, and 1995] and/or CLP-like data packages). This approach ensures that the data from this project can be compared to other datasets. Based on the 
minimum comparability requirements specified in the Industrial Sites QAPP (DOE/NV, 1996), all requirements were met.

Sample-handling documentation, laboratory nonconformance reports, and the precision and accuracy of QC sample results were evaluated for their effect on the results of the associated environmental soil samples. The environmental sample results were then qualified according to processes outlined in the following section. Documentation of the data qualifications resulting from these reviews is retained in project files.

\section{A.4.6 Data Evaluations}

All laboratory data from samples collected at the T-Tunnel Muckpile have been evaluated for data quality according to EPA Functional Guidelines (EPA, 1994a and 1999). These guidelines were implemented in a tiered process and are presented in the following text. Modifications to the laboratory-generated qualifiers were required to account for estimated values and associated blank contamination. No data rejected during the data evaluation process were used to reach the conclusions presented in the CADD. Only validated data, whether estimated (i.e., J-qualified) or not, were used in reaching conclusions.

Changes resulting from the data evaluation process are documented in project files and summarized in memoranda for each sample delivery group (SDG). These memoranda are maintained in the project files.

\section{A.4.6.1 Tier I}

Tier I evaluation for chemical analysis examines (but is not limited to):

- Sample count/type consistent with chain of custody

- Analysis count/type consistent with chain of custody

- Correct sample matrix

- Significant problems stated in cover letter or case narrative

- Completeness of certificates of analysis

- Completeness of CLP or CLP-like packages

- Completeness of signatures, dates, and times on chain of custody

- Condition upon receipt variance form included

- Requested analyses performed on all samples

- Date received/analyzed given for each sample

- Correct concentration units indicated

- Correct detection limits achieved 
- Electronic data transfer supplied

- Results reported for field and laboratory QC samples

- Whether or not the deliverable met the overall objectives of the project

Tier I data evaluations are summarized in a memorandum for each SDG showing results and qualifiers that were changed and the reason for these changes.

\section{A.4.6.2 Tier II}

Tier II evaluation for chemical analysis examines (but is not limited to):

- Sample date, preparation date, and analysis date for each sample

- Holding time criteria met

- QC batch association for each sample

- Cooler temperature upon receipt

- Sample $\mathrm{pH}$ for aqueous samples, as required

- Detection limits properly adjusted for dilution, as required

- Blank contamination evaluated and applied to sample results/qualifiers

- Matrix spike/MSD percent recoveries (\%R) and RPDs evaluated and applied to laboratory results/qualifiers

- Field duplicate RPDs evaluated and applied to laboratory results/qualifiers

- Laboratory duplicate RPDs evaluated and applied to laboratory results/qualifiers

- Surrogate $\%$ Rs evaluated and applied to laboratory results/qualifiers

- Laboratory control sample \%Rs evaluated and applied to laboratory results/qualifiers

- Mass spectrometer tuning criteria

- Initial and continuing calibration verification

- Internal standard evaluation

- Organic compound quantitation 
- Inductively coupled plasma (ICP) interference check sample evaluation

- Graphite furnace atomic absorption QC

- ICP serial dilution effects

Tier II data evaluations are summarized in a memorandum for each SDG showing results and qualifiers that were changed and the reason for these changes. None of the data qualifies were changed as a result of the Tier II validation.

\section{A.4.6.3 Tier III}

Additional data quality considerations included in EPA data review functional guidelines are evaluated as a third party Tier III review. Tier III review of chemical results include the following additional evaluations:

- Recalculation of all laboratory results from raw data

Tier III review was performed on at least 5 percent of the analytical data. A report of the findings has been issued and included in the project files. None of the data qualifies were changed as a result of the Tier III validation.

\section{A.4.7 Quality Control Samples}

Twenty-seven QC samples (i.e., trip blanks, equipment rinsate blanks, field duplicates, and MS/MSDs) were collected and submitted for laboratory analysis, as shown in Table A.3-1. The blanks and duplicates were assigned individual sample numbers and sent to the laboratory "blind." Additional samples were selected by the laboratory to be analyzed as laboratory replicates, duplicates, matrix duplicates, and MS/MSDs. Documentation related to the collection and analysis of these samples is retained in project files.

\section{A.4.7.1 Field Quality Control Samples}

Equipment rinsate blanks were analyzed for the parameters listed on Table A.3-2 (trip blanks were analyzed for VOCs only) and showed contamination associated with common laboratory artifacts (acetone, methylene chloride, and phthalate esters as defined in the EPA Functional Guidelines [EPA, 1994a and 1999]). These blank detections were used to qualify the results of the associated environmental samples according to EPA Functional Guidelines. 
According to the EPA Functional Guidelines, no qualification action is taken if a compound is found in an associated blank, but not in the sample, or if a compound is found in the sample, but not in an associated blank. The action taken when a compound is detected in both the sample and the associated blank varies depending upon the analyte involved and is known as "The 5X/10X Rule."

For most VOCs, SVOCs, and TPH-DRO analyses, an analyte detected in the sample above the instrument detection limits, that was also detected in an associated blank, is qualified as undetected (U) if the sample concentration is less than five times $(5 \mathrm{X})$ the blank concentration. For the common laboratory contaminants (methylene chloride, acetone, cyclohexane, 2-butanone [methylethyl ketone], and phthalate esters [especially bis(2-ethylhexyl) phthalate]), the factor is raised to ten times $(10 \mathrm{X})$ the blank concentration. The sample result is elevated to the quantitation limit/sample detection limit, if it is not already reported at that level. For inorganics (metals), sample results concentrations detected above the instrument detection limit but less than five times $(5 \mathrm{X})$ the amount found in an associated blank are qualified as undetected (U). There are no documented common metallic laboratory contaminants as compared to VOCs and SVOCs, so the sample result is never altered using a "10X rule."

Documentation of the data qualifications resulting from the application of these guidelines is retained in project files as both hard copy and electronic media.

Three field duplicate soil samples were sent as blind samples to the laboratory to be analyzed for the analytical parameters listed in Table A.3-2. For these samples, the duplicate results precision (i.e., relative percent differences between the environmental sample results and their corresponding field duplicate sample results) were compared to criteria set forth in the EPA Functional Guidelines (EPA, 1994a and 1999), and the associated environmental sample results were qualified accordingly.

The EPA Functional Guidelines give no required review criteria for field duplicate analyses comparability, but allow the data reviewer to exercise professional judgment. Both detections and nondetections are qualified as estimated ( $\mathrm{J}$ and $\mathrm{UJ}$, respectively) if the relative percent difference between an environmental sample and its field duplicate fall outside established criteria.

Three field samples were selected for use as MS/MSD samples. The $\% \mathrm{R}$ of these samples (a measure of accuracy) and the RPDs in these sample results (a measure of precision) were 
compared to EPA Functional Guideline (EPA, 1994a and 1999) criteria, and the results were used to qualify associated environmental sample results accordingly.

The EPA Functional Guidelines for review of organic data state that no data qualification action is taken on the basis of MS/MSD results alone. The data reviewer exercises professional judgment in considering these results in conjunction with the results of laboratory control samples and other QC criteria in applying qualifiers to the data. Generally, if recovery criteria are greater than the upper acceptance limit, then positive sample results for the affected compounds are qualified as estimated $(\mathrm{J})$, and nondetections are not qualified. If recovery criteria are less than the lower acceptance limit, then positive sample results for the affected compounds are qualified as estimated $(\mathrm{J})$ and nondetections are qualified as unusable $(\mathrm{R})$. The relative percent difference results of MS/MSD samples that fall outside established criteria are applied to qualify detections and nondetections as estimated ( $\mathrm{J}$ and $\mathrm{UJ}$, respectively).

The EPA Functional Guidelines for inorganic data review allow professional judgment to be applied in evaluating the results of matrix spikes (EPA, 1994a). Generally, if spike recoveries are greater than the upper acceptance limit, nondetections are acceptable for use. If spike recoveries are greater than the upper acceptance limit or less than the lower acceptance limit, positive results are qualified as estimated (J). If spike recovery falls within the 30 to 74 percent, nondetections are qualified as estimated (UJ), respectively. If spike recoveries are grossly low (less than 30 percent), positive results are unqualified, and nondetections are unusable (R). The relative percent difference between the environmental sample and its laboratory duplicate are compared to established criteria to qualify detections and nondetections as estimated (J and UJ, respectively).

\section{A.4.7.2 Laboratory Quality Control Samples}

Analysis of method QC blanks and laboratory control samples was performed for each parameter analyzed by Paragon Analytics, Inc. In addition, laboratory duplicate analysis was performed on several environmental samples per SDG. The results of these analyses were used to qualify associated environmental sample results according to EPA Functional Guidelines (EPA, 1994a and 1999), as discussed above.

\section{A.4.8 Nonconformances and Field Deficiencies}

No laboratory deficiencies were identified for this project. No field deficiencies were identified for this project. 


\section{A.5.0 Summary}

Analysis of the data generated from sampling activities conducted during corrective action investigation activities conducted at the T-Tunnel Muckpile indicates the following:

- Preliminary action levels were not exceeded for total VOCs or total SVOCs for any of the samples collected at the T-Tunnel Muckpile site.

- Preliminary action levels were not exceeded for RCRA metals except arsenic. Arsenic concentrations were detected above the industrial PRG levels in many samples collected including the investigation background samples. However, these concentrations were below the $23 \mathrm{mg} / \mathrm{kg}$ average for the NTS (Moore, 1999).

- Several isotopes associated with weapons testing were found in the surface soils of the muckpile in concentrations greater than background. These isotopes included Cs-137, Am-241, Pu-238, Pu-239, Sb-125, and Sr-90.

- Total petroleum hydrocarbons-DRO was detected at four locations near the surface in concentrations that exceeded the preliminary action level. The maximum detected concentration of TPH-DRO in any sample was $1,200 \mathrm{mg} / \mathrm{kg}$. However, none of the hazardous constituents found in TPH-DRO exceeded their PALs, so TPH-DRO is not considered a COC.

In summary, the only COCs identified in the muckpile are the radionuclides Cs-137, Am-241, $\mathrm{Pu}-238, \mathrm{Pu}-239$, Sb-125, and Sr-90. 


\section{A.6.0 References}

ASTM, see American Society for Testing and Materials.

American Society for Testing and Materials. 1995. Standard Test Method for Strontium-90 in Water, ASTM D 5811-95. Philadelphia, PA.

CFR, see Code of Federal Regulations.

Code of Federal Regulations. 1996. Title 40 Part 260-282, "RCRA Regulations." Washington, DC: Government Printing Office.

DOE, see U.S. Department of Energy.

DOE/NV, see U.S. Department of Energy, Nevada Operations Office.

DTRA, see Defense Threat Reduction Agency.

Defense Threat Reduction Agency. 2000a. Corrective Action Investigation Plan for Corrective Action Unit 476: Area 12 T-Tunnel Muckpile, Nevada Test Site, Rev. 0. Prepared by IT Corporation. Las Vegas, NV.

Defense Threat Reduction Agency. 2000b. Field Instruction for T-Tunnel Muckpile Characterization, Rev. 0. Prepared by IT Corporation. Las Vegas, NV.

EPA, see U.S. Environmental Protection Agency.

FFACO, see Federal Facility Agreement and Consent Order.

Federal Facility Agreement and Consent Order. 1996 (as amended). Agreed to by the State of Nevada, the U.S. Department of Energy, and the U.S. Department of Defense. Las Vegas, NV.

Gilbert, R.O. 1987. Statistical Methods for Environmental Pollution Monitoring. Chapter 4. New York, NY: Van Nostrand Reinhold.

ITLV, see IT Corporation, Las Vegas.

IT Corporation, Las Vegas. 2000. ITLV Site-Specific Health and Safety Plan for DTRA T-Tunnel Muckpile Characterization, March. Las Vegas, NV.

Moore, J. 1999. Memorandum to M. Todd (ITLV) entitled, "Background Concentrations for NTS and TTR Soil Samples," Las Vegas, NV.

U.S. Department of Energy, Nevada Operations Office. 1996. Industrial Sites Quality Assurance Project Plan, Nevada Test Site, Nevada, DOE/NV--372. Las Vegas, NV. 
U.S. Department of Energy. 1997. Environmental Measurements Laboratory Procedure Manual, 28th Edition, February, HASL-300. Washington, DC.

U.S. Environmental Protection Agency. 1980. Prescribed Procedures for Measurement of Radioactivity in Drinking Water. Washington, DC.

U.S. Environmental Protection Agency. 1987. Data Quality Objectives for Remedial Response Activities, EPA/540/G-87-003. Washington, DC.

U.S. Environmental Protection Agency. 1994a. Contract Laboratory Program National Functional Guidelines for Inorganic Data Review, EPA 540/R-94/013. Washington, DC.

U.S. Environmental Protection Agency. 1994b. Contract Laboratory Program Statement of Work for Organic Analysis, EPA 540/R-94/073. Washington, DC.

U.S. Environmental Protection Agency. 1994c. Contract Laboratory Program Statement of Work for Inorganic Analysis, EPA 540/R-94/076. Washington, DC.

U.S. Environmental Protection Agency. 1995. Methods and Contract Laboratory Program Scope of Work (SOW), EPA 540/R-95/121. Washington, DC.

U.S. Environmental Protection Agency. 1996. Test Methods for Evaluating Solid Waste, Physical/Chemical Methods, SW-846, CD ROM. Washington, DC.

U.S. Environmental Protection Agency. 1999. Contract Laboratory Program National Functional Guidelines for Organic Data Review, EPA 540/R-94/012. Washington, DC. 
Appendix B

\section{Data Quality Objective Process and Methodology}




\section{B.1.0 Data Quality Objective Process and Methodology}

Note: These DQOs were presented in the CAU 476 CAIP issued March 2, 2000. This was the second muckpile characterization conducted by DTRA.

The use of DQOs is part of the technical strategy found in Appendix VI of the FFACO (1996).

The DQO process is a systematic planning tool used to establish criteria for data quality and for developing the T-Tunnel Muckpile data collection program. This iterative, seven-step process results in a design to collect the right type, quality, and quantity of data needed to support a course of action for the site. The DQOs are designed to provide a means to determine what type of data need to be collected, and to ensure that the data collected are scientifically sound, defensible, and of documented quality. The DQOs are used to design a data collection program that will satisfy these goals. The DQOs described in this CAIP were modeled after those established for the N-Tunnel CAIP (DTRA, 1999).

The seven steps in the DQO process are:

- Statement of the problem

- Identification of the decision

- Identification of inputs to the decision

- Definition of study boundaries

- Determination of decision rules

- Specification of decision error limits

- Optimization of the design

The seven steps and their application to the T-Tunnel Muckpile are described in the following subsections.

\section{B.1.1 Problem Statement}

The problem is to determine whether materials comprising the T-Tunnel Muckpile contain COPCs at sufficiently high concentrations and volumes to require a corrective action.

\section{B.1.2 Definition of the Decision}

The primary decision is whether concentrations of COPCs in the muckpile exceed the EPA Region IX PRGs for Industrial Sites (EPA, 1999), contain TPH contamination at levels 
exceeding the NAC limits, or contain radioactive contamination exceeding background levels or above levels listed in Off-Site Radiation Exposure Review Project (ORERP), Phase II Soils Programs (McArthur and Miller, 1989).

\section{B.1.3 Identification of Inputs to the Decision}

Decisions regarding the sampling approach depend foremost upon a reasonable conceptual model (Section 3.1 of the T-Tunnel CAIP [DTRA, 2000]). The conceptual model provides a basis for developing an approach to the investigation and, ultimately, the course of action that will be taken for the site. The conceptual model of the muckpile will be tested using environmental sampling. This sampling program is described in more detail in Section 4.0 of the T-Tunnel CAIP (DTRA, 2000). If successful, the model will also serve as a guide to decisions concerning a subsequent course of action, if one is needed for the site.

\section{B.1.4 Definition of Study Boundaries}

The physical boundaries of the study area are defined in the $\mathrm{x}$ and $\mathrm{y}$ dimensions by the area extent of the T-Tunnel Muckpile. These boundaries are identifiable based on an inspection of the site, and by comparing DTRA's historical aerial photograph of the T-Tunnel area with the Rainier Mesa 7.5-minute scale topographic map (USGS, 1986). In the vertical (z) dimension, the study area extends to native material depending on whether it is bedrock or alluvium. The study area also includes background sample locations outside, but adjacent to, the muckpile boundaries.

\section{B.1.5 Determination of Decision Rules}

The laboratory analytical results will provide information to assist in determining the need for further action, if any, required at this site. Further action may be needed if sufficient quantities of COPCs are identified above the following PALs:

- $100 \mathrm{mg} / \mathrm{kg}$ TPH in soil, per the "Water Controls" (NAC, 1999)

- $\quad$ EPA Region IX PRGs (EPA, 1999) for industrial soils

- Radionuclide activities above natural background levels or above levels listed in the ORERP, Phase II Soils Programs Report (McArthur and Miller, 1989) 
For any COCs not addressed by the above standards, site-specific concentration limits will be derived based on the proposed RCRA Subpart S rules for corrective actions (Federal Register, 1990).

\section{B.1.6 Specification on Decision Error Limits}

The baseline condition, or null hypothesis, assumed for this site is that COPCs above action levels will be identified in the muckpile. The alternate hypothesis is that COPCs above action levels will not be identified. Based on these hypotheses, there are two types of decision errors are possible in implementing the CAIP. These errors are described as a false positive and a false negative. The CAIP was designed to minimize both types of errors.

The consequences of a false positive are: (1) that the corrective action could be needlessly expanded or encompass a greater quantity of media than is necessary, and (2) media incorrectly judged to be contaminated could be treated as regulated waste rather than unregulated waste. Both of these consequences could lead to increased corrective action and waste disposal costs.

The consequences of a false negative are: (1) regulated contaminants might not be appropriately addressed by corrective action or treatment activities, (2) contamination could remain in place, and (3) contaminated media might be disposed of improperly. These consequences could result in unacceptable risks to human health and the environment and potential fines from regulatory agencies.

The sampling program for T-Tunnel Muckpile was designed to provide preliminary data to allow statistical determination of whether enough samples were collected to sufficiently characterize the site. The determination will be made using the procedures described in Chapter 9 of the EPA publication SW-846, Test Methods for Evaluating Solid Waste, Physical/Chemical Methods (EPA, 1996). The mean concentration (or activity) and standard deviation of each targeted analyte in the muckpile soils was used to calculate the number of samples necessary to make the determination with a 90 percent confidence level.

\section{B.1.7 Optimization of the Design for Obtaining Data}

The sampling program has been optimized by determining the location and number of samples to collect and by determining which parameters should be analyzed. The COPCs for CAU 476 are TPH-DRO, VOCs, SVOCs, RCRA metals, and radionuclides. All environmental samples will 
be analyzed for these parameters, with the exception of the background samples which will be analyzed for radionuclides and RCRA metals.

Sample locations identified on the surface of the muckpile are biased to reflect areas where work processes may have impacted the muckpile surface soils. All areas of the T-Tunnel Muckpile near surface and subsurface will not have the same potential for radionuclide contamination, and therefore will not require the same level of survey coverage in order to achieve the required 90 percent confidence level. The resources necessary to investigate the muckpile are more efficiently utilized by a survey designed so areas with higher potential for contamination will receive a higher degree of sampling effort. Additionally, field-screening results for VOCs and radionuclides will be used to identify potentially contaminated media from the boreholes. 


\section{B.2.0 References}

DTRA, see Defense Threat Reduction Agency.

Defense Threat Reduction Agency. 1999. Corrective Action Investigation Plan for Corrective Action Unit 477: Area 12 N-Tunnel Muckpile, Nevada Test Site, Rev. 0. Prepared by IT Corporation. Las Vegas, NV.

Defense Threat Reduction Agency. 2000. Corrective Action Investigation Plan for Corrective Action Unit 476: Area 12 T-Tunnel Muckpile, Nevada Test Site, Rev. 0. Prepared by IT Corporation. Las Vegas, NV.

EPA, see U.S. Environmental Protection Agency.

FFACO, see Federal Facility Agreement and Consent Order.

Federal Facility Agreement and Consent Order. 1996 (as amended). Agreed to by the State of Nevada, the U.S. Department of Energy, and the U.S. Department of Defense.

Las Vegas, NV.

Federal Register. 1990. Proposed RCRA Subpart S Rules for Corrective Actions, v. 55, no. 145.

McArthur, R.D. and F.L. Miller, Jr. 1989. Off-Site Radiation Exposure Review Project, Phase II Soils Programs, DOE/NV/10384-23. Las Vegas, NV: Desert Research Institute.

NAC, see Nevada Administrative Code.

Nevada Administrative Code. 1999. NAC 445A.227, "Water Controls.” Carson City, NV.

U.S. Environmental Protection Agency. 1996. Test Methods for Evaluating Solid Waste, Physical/Chemical Methods, Rev. 1, SW-846, $3^{\text {rd }}$ Edition, CD ROM Version 2.0 with updates, PB97-501928GEI. Office of Solid Waste and Emergency Response. Washington, DC.

U.S. Environmental Protection Agency. 1999. Letter from S.J. Smucker to PRG Table Mailing List, entitled "Region IX Preliminary Remediation Goals." 1 October. San Francisco, CA.

U.S. Geological Survey. 1986. "Rainier Mesa, Nevada," 7.5-Minute Topographic Quadrangle map, Scale 1:24,000. 


\section{Appendix C}

\section{Data Assessment}




\section{C.1.0 Data Assessment}

Note: After the characterization was completed and the initial CAI summary and CADD were prepared, some of the guidance documents were updated. The data assessment has been updated to comply with the updated documents and new PALs.

The DQA process is the scientific evaluation of the investigation results to determine whether the DQO criteria established in the CAU 476 CAIP were met and whether the DQO decisions can be supported at the desired level of confidence. The DQO process ensures that the right type, quality, and quantity of data will be available to support the resolution of the decisions at an appropriate level of confidence. Using both the DQO and DQA processes helps to ensure that the DQO decisions are sound and defensible, and that the 90 percent level of confidence agreed to in the CAIP was achieved.

The DQA involves five steps that begin with a review of the DQOs and end with an answer to the DQO decisions. The five steps are briefly summarized below.

Step 1: Review the DQOs and Sampling Design - Review the DQO process to provide context for analyzing the data. State the primary statistical hypotheses; confirm the limits on the decision errors for committing false rejection (Type I) or false acceptance (Type II) decision errors; and review any special features, potential problems, or deviations to the sampling design.

Step 2: Conduct a Preliminary Data Review - The preliminary data review involves reviewing QA reports and inspecting the data both numerically and graphically, validating and verifying the data to ensure that the measurement systems performed in accordance with the criteria specified, and using the validated data to determine whether the quality of the data is satisfactory.

Step 3: Select the Test - Select the test based on the population of interest, population parameter, and the hypotheses. Identify the key underlying assumptions that could cause a change in one of the DQO decisions.

Step 4: Verify the Assumptions - Perform tests of assumptions. If data are missing or are censored, determine the impact on the DQO decision error.

Step 5: Draw Conclusions from the Data - Perform the calculations required for the test. 


\section{C.1.1 Review the DQOs and Sampling Design}

This section contains a review of the DQO process presented in the CAU 476 CAIP (DTRA, 2000) and Appendix B of this document. The DQO decisions are presented with the DQO provisions for limiting false negative or false positive decision errors. Special features, potential problems, or any deviations from the sampling design are also presented.

\section{C.1.1.1 Review DQOs}

The decision statement as presented in the CAU 476 CAIP is: "Whether concentrations of COPCs in the muckpile exceed the EPA Region 9 Preliminary Remediation Goals for Industrial Sites (EPA, 2004), contain TPH contamination at levels exceeding the Nevada Administrative Code limits, or contain radioactive contamination exceeding background levels or the levels listed in the Off-Site Radiation Exposure Review Project, Phase II Soils Programs Report (McArthur and Miller, 1989).”

\section{DQO Provisions To Limit False Negative Decision Error}

A false negative decision error (where consequences are more severe) was controlled by meeting the following criteria:

1. Having a high degree of confidence that the combination of random and biased sampling strategies will identify COCs if present in the CASs.

2. Having a high degree of confidence that analyses conducted will be sufficient to detect any COCs present in the samples.

3. Having a high degree of confidence that the data are of sufficient quality and completeness.

\section{Criterion 1:}

The following methods (stipulated in the CAU 476 DQOs [DTRA, 2000] and agree to by NDEP) were used in selecting the sample locations:

- Random locations to collect soil samples from the muckpile.

- Biased locations based on professional judgment and site knowledge to collect soil samples from the muckpile. 
This provides a high degree of confidence that sampling will detect any COCs that may be present.

\section{Criterion 2:}

All samples were analyzed using the analytical methods listed in Table D-1 of the CAIP. Table C.1-1 provides a reconciliation of environmental samples analyzed to the planned analytical program. Samples were analyzed for all of the analytical methods specified in the CAIP (DTRA, 2000).

Table C.1-1

CAU 476 Number of Soil Samples Submitted per Analyte

\begin{tabular}{|c|c|c|c|c|c|c|c|}
\hline & \multicolumn{7}{|c|}{ Analytes } \\
\hline & $\begin{array}{l}\text { Ủ } \\
\stackrel{3}{3}\end{array}$ & 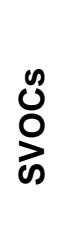 & 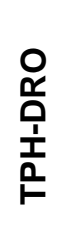 & $\begin{array}{l}\frac{n}{\mathbb{T}} \\
\stackrel{\mathbb{U}}{\Sigma}\end{array}$ & 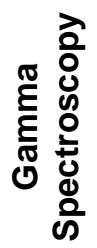 & 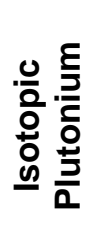 & 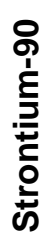 \\
\hline Muckpile Soil & 22 & 22 & 22 & 22 & 22 & 5 & 5 \\
\hline Native Soil & 20 & 20 & 20 & 20 & 20 & 4 & 4 \\
\hline Biased Shallow Soil & 9 & 9 & 9 & 9 & 9 & 5 & 5 \\
\hline Background & 0 & 0 & 0 & 3 & 3 & 3 & 3 \\
\hline
\end{tabular}

$\mathrm{DRO}=$ Diesel-range organics

SVOC = Semivolatile organic compound

$\mathrm{TPH}=$ Total petroleum hydrocarbons

VOC $=$ Volatile organic compound

Sample results were assessed against the DQI of sensitivity as defined in the Industrial Sites QAPP (NNSA/NV, 2002). The sensitivity acceptance criteria defined in the CAIP is that analytical detection limits will be less than the corresponding action level. This goal was not achieved for the chemical analyses listed in Table C.1-2. All radiological analytes met the sensitivity goal. Results not meeting the sensitivity goal were not used in making DQO decisions and will therefore be considered as rejected data. 


\section{Table C.1-2 \\ Chemical Analytes Failing Sensitivity Criteria for CAU 476}

(Page 1 of 2)

\begin{tabular}{|c|c|c|c|c|}
\hline Sample Number & Parameter & $\begin{array}{l}\text { Result } \\
(\mu \mathrm{g} / \mathrm{kg})\end{array}$ & $\begin{array}{c}\text { Detection } \\
\text { Limit } \\
(\mu \mathrm{g} / \mathrm{kg})\end{array}$ & $\begin{array}{l}2004 \text { Industrial } \\
\text { PRG ( } \mu \mathrm{g} / \mathrm{kg})\end{array}$ \\
\hline TS-18-3.0 & Benzo(a)Pyrene & 4,100 & 1,600 & 210 \\
\hline TS-S1-0.5 & Benzo(a)Pyrene & 4,000 & 1,500 & 210 \\
\hline TS-18-3.0 & Benzo(b)Fluoranthene & 4,100 & 2,200 & 2,100 \\
\hline TS-S1-0.5 & Benzo(b)Fluoranthene & 4,000 & 2,100 & 2,100 \\
\hline QTS-05-24 & Dibenzo(a,h)Anthracene & 430 & 240 & 210 \\
\hline QTS-06-12 & Dibenzo(a,h)Anthracene & 400 & 230 & 210 \\
\hline TS-02-56.5 & Dibenzo(a,h)Anthracene & 370 & 210 & 210 \\
\hline TS-02-8.5 & Dibenzo(a,h)Anthracene & 420 & 240 & 210 \\
\hline TS-03-09 & Dibenzo(a,h)Anthracene & 420 & 240 & 210 \\
\hline TS-03-61.5 & Dibenzo(a,h)Anthracene & 370 & 210 & 210 \\
\hline TS-04-36.5 & Dibenzo(a,h)Anthracene & 410 & 240 & 210 \\
\hline TS-04-63.5 & Dibenzo(a,h)Anthracene & 370 & 210 & 210 \\
\hline TS-04A-58 & Dibenzo(a,h)Anthracene & 430 & 240 & 210 \\
\hline TS-04A-68.5 & Dibenzo(a,h)Anthracene & 370 & 210 & 210 \\
\hline TS-05-24 & Dibenzo(a,h)Anthracene & 430 & 240 & 210 \\
\hline TS-05-51.5 & Dibenzo(a,h)Anthracene & 360 & 210 & 210 \\
\hline TS-06-12 & Dibenzo(a,h)Anthracene & 400 & 230 & 210 \\
\hline TS-06-47.5 & Dibenzo(a,h)Anthracene & 390 & 220 & 210 \\
\hline TS-07-13 & Dibenzo(a,h)Anthracene & 420 & 240 & 210 \\
\hline TS-08-22 & Dibenzo(a,h)Anthracene & 430 & 250 & 210 \\
\hline TS-08-38 & Dibenzo(a,h)Anthracene & 370 & 210 & 210 \\
\hline TS-09-30 & Dibenzo(a,h)Anthracene & 450 & 260 & 210 \\
\hline TS-09-35 & Dibenzo(a,h)Anthracene & 380 & 220 & 210 \\
\hline TS-09-46 & Dibenzo(a,h)Anthracene & 390 & 220 & 210 \\
\hline TS-10-12 & Dibenzo(a,h)Anthracene & 410 & 230 & 210 \\
\hline TS-10-50 & Dibenzo(a,h)Anthracene & 370 & 210 & 210 \\
\hline TS-11-31.5 & Dibenzo(a,h)Anthracene & 400 & 230 & 210 \\
\hline TS-11-39.5 & Dibenzo(a,h)Anthracene & 430 & 240 & 210 \\
\hline TS-11-51.5 & Dibenzo(a,h)Anthracene & 370 & 210 & 210 \\
\hline TS-12-14 & Dibenzo(a,h)Anthracene & 400 & 230 & 210 \\
\hline TS-12-24.5 & Dibenzo(a,h)Anthracene & 370 & 210 & 210 \\
\hline TS-13-14 & Dibenzo(a,h)Anthracene & 390 & 220 & 210 \\
\hline TS-13-41 & Dibenzo(a,h)Anthracene & 370 & 210 & 210 \\
\hline TS-14-08 & Dibenzo(a,h)Anthracene & 400 & 230 & 210 \\
\hline TS-14-24 & Dibenzo(a,h)Anthracene & 370 & 210 & 210 \\
\hline
\end{tabular}




\section{Table C.1-2 \\ Chemical Analytes Failing Sensitivity Criteria for CAU 476}

(Page 2 of 2)

\begin{tabular}{|c|c|c|c|c|}
\hline Sample Number & Parameter & $\begin{array}{l}\text { Result } \\
(\mu \mathrm{g} / \mathrm{kg})\end{array}$ & $\begin{array}{c}\text { Detection } \\
\text { Limit } \\
(\mu \mathrm{g} / \mathrm{kg})\end{array}$ & $\begin{array}{c}2004 \text { Industrial } \\
\text { PRG }(\mu \mathrm{g} / \mathrm{kg})\end{array}$ \\
\hline TS-15-11.5 & Dibenzo(a,h)Anthracene & 410 & 240 & 210 \\
\hline TS-15-29 & Dibenzo(a,h)Anthracene & 380 & 210 & 210 \\
\hline TS-16-11 & Dibenzo(a,h)Anthracene & 380 & 220 & 210 \\
\hline TS-16-17 & Dibenzo(a,h)Anthracene & 380 & 220 & 210 \\
\hline TS-17-25 & Dibenzo(a,h)Anthracene & 370 & 210 & 210 \\
\hline TS-17-9.5 & Dibenzo(a,h)Anthracene & 400 & 230 & 210 \\
\hline TS-18-20 & Dibenzo(a,h)Anthracene & 420 & 240 & 210 \\
\hline TS-18-3.0 & Dibenzo(a,h)Anthracene & 4,100 & 2,300 & 210 \\
\hline TS-19-35 & Dibenzo(a,h)Anthracene & 410 & 230 & 210 \\
\hline TS-19-4.0 & Dibenzo(a,h)Anthracene & 400 & 230 & 210 \\
\hline TS-20-20 & Dibenzo(a,h)Anthracene & 410 & 230 & 210 \\
\hline TS-S1-0.5 & Dibenzo(a,h)Anthracene & 4,000 & 2,300 & 210 \\
\hline TS-S1-2.5 & Dibenzo(a,h)Anthracene & 420 & 240 & 210 \\
\hline TS-S2-0.5 & Dibenzo(a,h)Anthracene & 400 & 230 & 210 \\
\hline TS-S3-01 & Dibenzo(a,h)Anthracene & 410 & 230 & 210 \\
\hline TS-S4-0.5 & Dibenzo(a,h)Anthracene & 420 & 240 & 210 \\
\hline TS-S5-0.5 & Dibenzo(a,h)Anthracene & 400 & 230 & 210 \\
\hline TS-S6-0.5 & Dibenzo(a,h)Anthracene & 400 & 230 & 210 \\
\hline TS-S7-01 & Dibenzo(a,h)Anthracene & 420 & 240 & 210 \\
\hline TS-18-3.0 & Hexachlorobenzene & 4,100 & 1,700 & 1,100 \\
\hline TS-S1-0.5 & Hexachlorobenzene & 4,000 & 1,700 & 1,100 \\
\hline TS-18-3.0 & Indeno(1,2,3-cd)Pyrene & 4,100 & 2,400 & 2,100 \\
\hline TS-S1-0.5 & Indeno(1,2,3-cd)Pyrene & 4,000 & 2,400 & 2,100 \\
\hline TS-08-22 & N-Nitroso-Di-N-Propylamine & 430 & 250 & 250 \\
\hline TS-09-30 & N-Nitroso-Di-N-Propylamine & 450 & 260 & 250 \\
\hline TS-18-3.0 & N-Nitroso-Di-N-Propylamine & 4,100 & 2,300 & 250 \\
\hline TS-S1-0.5 & N-Nitroso-Di-N-Propylamine & 4,000 & 2,300 & 250 \\
\hline
\end{tabular}

PRG = Preliminary remediation goal

$\mu \mathrm{g} / \mathrm{kg}=$ Micrograms per kilogram

\section{Criterion 3:}

To satisfy the third criterion, the entire dataset, as well as individual sample results, were assessed against the acceptance criteria for the DQIs of precision, accuracy, comparability, completeness, and representativeness, as defined in the Industrial Sites QAPP 
(NNSA/NV, 2002). The DQI acceptance criteria for precision and accuracy are defined in Table D-1 of the CAIP (DTRA, 2000). The acceptance criteria for comparability, completeness, and representativeness are not specified in the CAIP. As presented in the following sections, the goals were met for each DQI except as noted.

\section{Precision}

The duplicate precision is evaluated using the relative percent difference (RPD) or normalized difference. For the purpose of determining the data precision of chemical analyses, the RPD between duplicate analyses was calculated. For radionuclides, the RPD was not calculated unless both the sample and its duplicate had a concentration of the target radionuclide exceeding five times their minimum detectable concentration. Otherwise, radionuclide duplicate results were evaluated using the normalized difference. No chemical analytes or radionuclides were qualified for precision.

Because all of the constituents exceed the acceptance criteria for precision, the dataset is determined to be acceptable for the DQI of precision.

\section{$\underline{\text { Accuracy }}$}

For the purpose of determining data accuracy of sample analyses, environmental soil samples were evaluated and incorporated into the accuracy calculation. The VOC and SVOC results qualified for accuracy were associated with MS and surrogate percent recoveries exceeding the QC limits. The samples that were qualified because of the MS recoveries are all biased high. Because the results are below the action level, there is no impact on the decision making process. The results qualified for surrogate percent recovery could be biased either high or low, but because the results (although estimated) are all significantly less than the action level, there is no reason to believe the estimated result will impact the decision making process. Table C.1-3 provides the evaluation results for the constituents qualified for accuracy. 


\section{Table C.1-3 \\ Accuracy Measurements}

(Page 1 of 3)

\begin{tabular}{|c|c|c|c|c|c|}
\hline Parameter & $\begin{array}{c}\text { CAS } \\
\text { Number }\end{array}$ & $\begin{array}{c}\text { User Test } \\
\text { Panel }\end{array}$ & $\begin{array}{c}\text { Number of } \\
\text { Analyses } \\
\text { Qualified }\end{array}$ & $\begin{array}{l}\text { Number of } \\
\text { Measurements } \\
\text { Performed }\end{array}$ & $\begin{array}{c}\text { Percent } \\
\text { within } \\
\text { Criteria }\end{array}$ \\
\hline $1,1,1,2$-Tetrachloroethane & $630-20-6$ & VOCs & 7 & 54 & 87 \\
\hline 1,1,1-Trichloroethane & $71-55-6$ & VOCs & 7 & 54 & 87 \\
\hline 1,1,2,2-Tetrachloroethane & $79-34-5$ & VOCs & 7 & 54 & 87 \\
\hline 1,1,2-Trichloroethane & $79-00-5$ & VOCs & 7 & 54 & 87 \\
\hline 1,1-Dichloroethane & $75-34-3$ & VOCs & 7 & 54 & 87 \\
\hline 1,1-Dichloroethene & $75-35-4$ & VOCs & 7 & 54 & 87 \\
\hline 1,1-Dichloropropene & $563-58-6$ & VOCs & 7 & 54 & 87 \\
\hline 1,2,3-Trichlorobenzene & $87-61-6$ & VOCs & 7 & 54 & 87 \\
\hline 1,2,3-Trichloropropane & $96-18-4$ & VOCs & 7 & 54 & 87 \\
\hline 1,2,4-Trichlorobenzene & $120-82-1$ & SVOCs & 7 & 54 & 87 \\
\hline 1,2,4-Trichlorobenzene & $120-82-1$ & VOCs & 7 & 54 & 87 \\
\hline 1,2,4-Trimethylbenzene & $95-63-6$ & VOCs & 7 & 54 & 87 \\
\hline 1,2-Dibromo-3-Chloropropane & $96-12-8$ & VOCs & 7 & 54 & 87 \\
\hline 1,2-Dibromoethane & $106-93-4$ & VOCs & 7 & 54 & 87 \\
\hline 1,2-Dichlorobenzene & $95-50-1$ & SVOCs & 7 & 54 & 87 \\
\hline 1,2-Dichlorobenzene & $95-50-1$ & VOCs & 7 & 54 & 87 \\
\hline 1,2-Dichloroethane & $107-06-2$ & VOCs & 7 & 54 & 87 \\
\hline 1,2-Dichloropropane & $78-87-5$ & VOCs & 7 & 54 & 87 \\
\hline 1,3,5-Trimethylbenzene & $108-67-8$ & VOCs & 7 & 54 & 87 \\
\hline 1,3-Dichlorobenzene & $541-73-1$ & SVOCs & 7 & 54 & 87 \\
\hline 1,3-Dichlorobenzene & $541-73-1$ & VOCs & 7 & 54 & 87 \\
\hline 1,3-Dichloropropane & $142-28-9$ & VOCs & 7 & 54 & 87 \\
\hline 1,4-Dichlorobenzene & $106-46-7$ & SVOCs & 7 & 54 & 87 \\
\hline 1,4-Dichlorobenzene & $106-46-7$ & VOCs & 7 & 54 & 87 \\
\hline 1-Chlorohexane & $544-10-5$ & VOCs & 7 & 54 & 87 \\
\hline 2,2-Dichloropropane & $594-20-7$ & VOCs & 7 & 54 & 87 \\
\hline 2-Butanone & $78-93-3$ & VOCs & 7 & 54 & 87 \\
\hline 2-Chlorotoluene & $95-49-8$ & VOCs & 7 & 54 & 87 \\
\hline 2-Hexanone & $591-78-6$ & VOCs & 7 & 54 & 87 \\
\hline 4-Chlorotoluene & $106-43-4$ & VOCs & 7 & 54 & 87 \\
\hline 4-Methyl-2-Pentanone & $108-10-1$ & VOCs & 7 & 54 & 87 \\
\hline
\end{tabular}


Table C.1-3

Accuracy Measurements

(Page 2 of 3 )

\begin{tabular}{|c|c|c|c|c|c|}
\hline Parameter & $\begin{array}{c}\text { CAS } \\
\text { Number }\end{array}$ & $\begin{array}{c}\text { User Test } \\
\text { Panel }\end{array}$ & $\begin{array}{c}\text { Number of } \\
\text { Analyses } \\
\text { Qualified }\end{array}$ & $\begin{array}{l}\text { Number of } \\
\text { Measurements } \\
\text { Performed }\end{array}$ & $\begin{array}{c}\text { Percent } \\
\text { within } \\
\text { Criteria }\end{array}$ \\
\hline Acetone & $67-64-1$ & VOCs & 7 & 54 & 87 \\
\hline Bromobenzene & $108-86-1$ & VOCs & 7 & 54 & 87 \\
\hline Bromochloromethane & $74-97-5$ & VOCs & 7 & 54 & 87 \\
\hline Bromodichloromethane & $75-27-4$ & VOCs & 7 & 54 & 87 \\
\hline Bromoform & $75-25-2$ & VOCs & 7 & 54 & 87 \\
\hline Bromomethane & $74-83-9$ & VOCs & 7 & 54 & 87 \\
\hline Carbon Disulfide & $75-15-0$ & VOCs & 7 & 54 & 87 \\
\hline Carbon Tetrachloride & $56-23-5$ & VOCs & 7 & 54 & 87 \\
\hline Chloroethane & $75-00-3$ & VOCs & 7 & 54 & 87 \\
\hline Chloroform & $67-66-3$ & VOCs & 7 & 54 & 87 \\
\hline Chloromethane & $74-87-3$ & VOCs & 7 & 54 & 87 \\
\hline Cis-1,2-Dichloroethene & $156-59-2$ & VOCs & 7 & 54 & 87 \\
\hline Cis-1,3-Dichloropropene & $10061-01-5$ & VOCs & 7 & 54 & 87 \\
\hline Dibromochloromethane & $124-48-1$ & VOCs & 7 & 54 & 87 \\
\hline Dibromomethane & $74-95-3$ & VOCs & 7 & 54 & 87 \\
\hline Dichlorodifluoromethane & $75-71-8$ & VOCs & 7 & 54 & 87 \\
\hline Ethylbenzene & $100-41-4$ & VOCs & 7 & 54 & 87 \\
\hline Hexachlorobutadiene & $87-68-3$ & SVOCs & 7 & 54 & 87 \\
\hline Hexachlorobutadiene & $87-68-3$ & VOCs & 7 & 54 & 87 \\
\hline lodomethane & $74-88-4$ & VOCs & 7 & 54 & 87 \\
\hline Isopropylbenzene & $98-82-8$ & VOCs & 7 & 54 & 87 \\
\hline M+P-Xylene & $136777-61-2$ & VOCs & 7 & 54 & 87 \\
\hline Methyl Tertiary Butyl Ether & $1634-04-4$ & VOCs & 7 & 54 & 87 \\
\hline Methylene Chloride & $75-09-2$ & VOCs & 7 & 54 & 87 \\
\hline N-Butylbenzene & $104-51-8$ & VOCs & 7 & 54 & 87 \\
\hline N-Propylbenzene & $103-65-1$ & VOCs & 7 & 54 & 87 \\
\hline Naphthalene & $91-20-3$ & SVOCs & 7 & 54 & 87 \\
\hline Naphthalene & $91-20-3$ & VOCs & 7 & 54 & 87 \\
\hline O-Xylene & $95-47-6$ & VOCs & 7 & 54 & 87 \\
\hline P-Isopropyltoluene & $99-87-6$ & VOCs & 7 & 54 & 87 \\
\hline Sec-Butylbenzene & $135-98-8$ & VOCs & 7 & 54 & 87 \\
\hline
\end{tabular}


Table C.1-3

Accuracy Measurements

(Page 3 of 3 )

\begin{tabular}{|c|c|c|c|c|c|}
\hline Parameter & $\begin{array}{c}\text { CAS } \\
\text { Number }\end{array}$ & $\begin{array}{c}\text { User Test } \\
\text { Panel }\end{array}$ & $\begin{array}{l}\text { Number of } \\
\text { Analyses } \\
\text { Qualified }\end{array}$ & $\begin{array}{l}\text { Number of } \\
\text { Measurements } \\
\text { Performed }\end{array}$ & $\begin{array}{c}\text { Percent } \\
\text { within } \\
\text { Criteria }\end{array}$ \\
\hline Styrene & $100-42-5$ & VOCs & 7 & 54 & 87 \\
\hline Tert-Butylbenzene & $98-06-6$ & VOCs & 7 & 54 & 87 \\
\hline Tetrachloroethene & $127-18-4$ & VOCs & 7 & 54 & 87 \\
\hline Trans-1,2-Dichloroethene & $156-60-5$ & VOCs & 7 & 54 & 87 \\
\hline Trans-1,3-Dichloropropene & $10061-02-6$ & VOCs & 7 & 54 & 87 \\
\hline Trichlorofluoromethane & $75-69-4$ & VOCs & 7 & 54 & 87 \\
\hline Trichlorotrifluoroethane & $76-13-1$ & VOCs & 7 & 54 & 87 \\
\hline Vinyl Acetate & $108-05-4$ & VOCs & 7 & 54 & 87 \\
\hline Vinyl Chloride & $75-01-4$ & VOCs & 7 & 54 & 87 \\
\hline Benzene & $71-43-2$ & VOCs & 14 & 54 & 74.1 \\
\hline Chlorobenzene & $108-90-7$ & VOCs & 14 & 54 & 74.1 \\
\hline Toluene & $108-88-3$ & VOCs & 14 & 54 & 74.1 \\
\hline Trichloroethene & $79-01-6$ & VOCs & 14 & 54 & 74.1 \\
\hline
\end{tabular}

CAS $=$ Chemical Abstracts Service

$S V O C=$ Semivolatile organic compound

$\mathrm{VOC}=$ Volatile organic compound

All of the VOC concentrations that qualified for accuracy still exceeded the 80 percent accuracy criteria specified in the QAPP except for benzene, chlorobenzene, toluene, and trichloroethene. These constituents only had an accuracy rate of 74 percent. However, all of these constituents, except for two trichloroethene samples, were listed as non-detects with detection limits between 0.0012 and 5 percent of the PAL. The two trichloroethene samples had estimated concentrations of 0.49 and 0.76 micrograms per kilogram $(\mu \mathrm{g} / \mathrm{kg}$ ), which are 0.45 and 0.69 percent of the PAL. This makes the likelihood of a false negative having an actual concentration above the PAL very small; therefore, these data can still be used to support the DQO decisions. None of the analytes qualified were considered to be suspected contaminants for this site. As the accuracy rate for all of the other constituents exceeds the acceptance criteria, the dataset is determined to be acceptable for the DQI of accuracy.

\section{Representativeness}

The DQO process, as identified in Section 3.0 of the CAU 476 CAIP (DTRA, 2000), was used to address sampling and analytical requirements for CAU 476. During this process, appropriate 
locations were selected that enabled the samples collected to be representative of the population parameters identified in the DQO (random locations and biased locations that were most likely to encounter contamination). Therefore, the analytical data acquired during the CAU $476 \mathrm{CAI}$ are considered to be representative of the population parameters.

\section{Comparability}

Field sampling, as described in the CAU 476 CAIP (DTRA, 2000), was performed and documented in accordance with approved procedures that are comparable to standard industry practices. Approved analytical methods and procedures were used to analyze, report, and validate the data. These are comparable to other methods used not only in industry and government practices, but most importantly are comparable to other investigations conducted at the NTS. Therefore, project datasets are considered comparable to other datasets generated using these same standardized DOE procedures, thereby meeting the DQO requirements. Also, standard, approved field and analytical methods ensure that data were appropriate for comparison to the investigation action levels specified in the CAIP (DTRA, 2000).

\section{Completeness}

The CAU 476 CAIP (DTRA, 2000) did not define criteria for completeness; therefore, the criteria of 80 percent of CAS-specific non-critical analytes identified in the CAIP having valid results and 100 percent of critical analytes having valid results will be used for the CAU 476 evaluation. Also, the dataset must be sufficiently complete to be able to support the DQO decisions. Critical analytes for CAU 476 were not defined, so the COCs identified from other investigated NTS muckpiles (arsenic, lead, TPH-DRO, Pu-239, Cs-137, and Co-60) have been defined as the critical analytes for CAU 476.

Rejected data (either qualified as rejected or data that failed the criterion of sensitivity) were not used in the resolution of DQO decisions and are not counted toward meeting the completeness acceptance criterion. The completeness for all critical chemical and radiological data was 100 percent; therefore, the dataset is considered complete for purposes of supporting the DQO decisions.

\section{DQO Provisions To Limit False Positive Decision Error}

The false positive decision error was controlled by assessing the potential for false positive analytical results. Quality assurance (QA)/quality control (QC) samples such as field blanks, trip blanks, laboratory control samples, and method blanks were used to determine whether a false 
positive analytical result may have occurred. Of the $34 \mathrm{QA} / \mathrm{QC}$ samples submitted, no false positive analytical results were detected.

Proper decontamination of sampling equipment and the use of certified clean sampling equipment and containers minimized the potential for cross contamination, which could lead to a false positive analytical result.

\section{C.1.1.2 Sampling Design}

The CAIP (DTRA, 2000) made the following commitments for sampling:

1. Background samples will be collected at undisturbed locations around the muckpile.

Result: Three locations were identified and sampled using hand tools. The samples were field screened and shipped to an off-site laboratory for analysis.

2. Random sampling will be conducted on the muckpile.

Result: Twenty random locations were identified and sampled using rotosonic drilling methods. The samples were field screened and shipped to an off-site laboratory for analysis.

3. Biased locations will be identified and drilled to investigate suspected surface contaminated areas.

Result: Eight biased locations were identified and sampled to investigate areas with visible staining or elevated radiological readings identified in the walkover surveys. The samples were field screened and shipped to an off-site laboratory for analysis.

\section{C.1.2 Conduct a Preliminary Data Review}

A preliminary data review was conducted by reviewing QA reports and inspecting the data. The contract analytical laboratories generate a QA non-conformance report when data quality does not meet contractual requirements. All data received from the analytical laboratories met contractual requirements, and no QA non-conformance reports were generated. Data were validated and verified to ensure that the measurement systems performed in accordance with the criteria specified. The validated dataset quality was found to be satisfactory. 


\section{C.1.3 Select the Test}

The CAIP (DTRA, 2000) committed to using the procedure described in Chapter 9 of the EPA SW-846 Method (EPA, 1999) to answer two questions: 1) Were enough samples collected to ensure a 90 percent confidence level in the mean COPC concentration and 2) Does the mean concentration exceed the regulatory threshold?

Because of the change in closure strategy agreed to by NDEP, DTRA, and DOE, National Nuclear Security Administration Nevada Site Office (NNSA/NSO), the regulatory threshold is now the risk-based FAL instead of the PALs discussed in the CAIP. Comparing the average concentration of the most prevalent contaminants to their PAL and, if they exceed the PAL, comparing them to their respective FALs will also be used to help answer the questions.

\section{C.1.4 SW-846 Evaluation}

To answer the first question, equation (8) of Table 9-1 in SW-846 was used. To answer the second question, equation (6) of Table 9-1 in SW-846 was used (EPA, 1999). Only results from random samples were used for this evaluation. These questions were answered for the critical analytes arsenic, Cs-137, lead, and TPH-DRO as they were the only critical analytes for which there were sufficient detections.

Question 1: "Were enough samples collected?" is answered by solving equation (8) of Table 9-1 in SW-846 for each analyte.

$$
\mathrm{n}=\mathrm{t}_{.20}{ }^{2} \times \mathrm{s}^{2} /(\mathrm{RT}-\overline{\mathrm{x}})^{2} \quad \text { where }
$$

(Equation 1)

$\mathrm{n}=$ minimum number of samples to ensure a 90 percent confidence level

$\mathrm{t}_{20}{ }^{2}=$ the square of the " $\mathrm{t}$ " value in Table 9-2, SW-846 for a one-tailed 90 percent confidence interval

$\mathrm{s}^{2}=$ variance in the concentration measured in the samples collected during characterization

RT $=$ regulatory threshold and is set to the limiting PRG established by the EPA for the COPC for the industrial land use. For TPH, the RT is $100 \mathrm{mg} / \mathrm{kg}$. For radionuclides, it is the U.S. Nuclear Regulatory Commission and National Council on Radiation Protection and Measurements screening levels 
$\bar{x}=$ the mean concentration of the COPC in the collected samples.

Question 2: "Does the mean concentration exceed the regulatory threshold?" is answered by solving equation (6) of Table 9-1 in SW-846 for each analyte.

$$
\mathrm{CI}=\overline{\mathrm{x}}+/-\left(\mathrm{t}_{.20} \times\left(\frac{\mathrm{s}}{\sqrt{n}}\right)\right) \quad \text { where }
$$

(Equation 2)

$\mathrm{CI}=$ confidence interval

$\bar{x} \quad=$ the mean concentration of the COPC in the collected samples

$\mathrm{n}=$ number of samples collected

$\mathrm{t}_{.20}=$ the " $\mathrm{t}$ " value in Table $9-2, \mathrm{SW}-846$ for a one-tailed 90 percent confidence interval and the appropriate degrees of freedom

$\mathrm{s}_{\overline{\mathrm{x}}} \quad=\quad$ variance in the concentration measured in the samples collected during characterization

The values used for the calculations and the results are presented in Table C.1-4.

Table C.1-4

SW-846 Evaluation of the Number of Samples and Comparison of $90 \%$ Confidence Level with the PAL

\begin{tabular}{|c|c|c|c|c|}
\hline Variable & Arsenic & Cs-137 & Lead & TPH-DRO \\
\hline \hline $\mathrm{T} .20$ & 1.328 & 1.393 & 1.328 & 1.328 \\
\hline $\mathrm{T}_{20}{ }^{2}$ & 1.763 & 1.940 & 1.763 & 1.763 \\
\hline $\mathrm{S}^{2}$ & 5.329 & 0.024 & $3,342.613$ & $5,508.574$ \\
\hline $\mathrm{RT}$ & $23 \mathrm{mg} / \mathrm{kg}$ & $12.2 \mathrm{pCi} / \mathrm{g}$ & $800 \mathrm{mg} / \mathrm{kg}$ & $100 \mathrm{mg} / \mathrm{kg}$ \\
\hline $\mathrm{Avg} \overline{\mathrm{x}}$ & $4.15 \mathrm{mg} / \mathrm{kg}$ & $0.198 \mathrm{pCi} / \mathrm{g}$ & $36.575 \mathrm{mg} / \mathrm{kg}$ & $28.003 \mathrm{mg} / \mathrm{kg}$ \\
\hline $\mathrm{n}$ collected & 20 & 10 & 20 & 20 \\
\hline $\mathrm{n}$ needed & $<1$ & $<1$ & $<1$ & 2 \\
\hline Upper Confidence Interval & $7.216 \mathrm{mg} / \mathrm{kg}$ & $0.416 \mathrm{pCi} / \mathrm{g}$ & $113.359 \mathrm{mg} / \mathrm{kg}$ & $126.564 \mathrm{mg} / \mathrm{kg}$ \\
\hline Upper Confidence>RT & No & No & No & Yes \\
\hline
\end{tabular}

Cs $=$ Cesium

$\mathrm{DRO}=$ Diesel-range organics

$\mathrm{TPH}=$ Total petroleum hydrocarbons

$\mathrm{mg} / \mathrm{kg}=$ Milligrams per kilogram

$\mathrm{PAL}=$ Preliminary action level

$\mathrm{pCi} / \mathrm{g}=$ Picocuries per gram

$\mathrm{RT}=$ Regulatory threshold 
Based on the results of the calculations, an adequate number of samples were collected to meet the 90 percent confidence level for characterization of the site for all four critical analytes. In comparing the 90 percent confidence level to the RT, the confidence level for arsenic, Cs-137, and lead are below the RT. The confidence level for TPH-DRO exceeds the RT by $26.5 \mathrm{mg} / \mathrm{kg}$ for the CAU 476 T-Tunnel Muckpile. Because the confidence level for TPH-DRO exceeds the RT, TPH-DRO will be moved to a Tier 2 analysis to determine whether it poses a threat to human health or the environment.

\section{C.1.5 Verify the Assumptions}

The results of the investigation support the assumptions identified in the CAU 476 CAIP and in Table C.1-5.

\section{Table C.1-5 \\ Key Assumptions}

\begin{tabular}{|c|c|}
\hline \multirow[t]{2}{*}{ Exposure Scenario } & $\begin{array}{l}\text { Exposure to contaminants is limited to industrial site workers, } \\
\text { construction/remediation workers, and military personnel conducting training. } \\
\text { Exposure could occur through ingestion, inhalation, external exposure, or } \\
\text { dermal contact. }\end{array}$ \\
\hline & $\begin{array}{l}\text { The investigation did not reveal any potential exposures that were not identified } \\
\text { in the conceptual site model (CSM). }\end{array}$ \\
\hline \multirow[t]{2}{*}{ Affected Media } & $\begin{array}{l}\text { Surface and subsurface soils in and below the muckpile. Contamination of } \\
\text { perched, deep, and regional groundwater is not a concern. }\end{array}$ \\
\hline & $\begin{array}{l}\text { The investigation results did not identify any affected media that were not } \\
\text { identified in the CSM. }\end{array}$ \\
\hline \multirow[t]{2}{*}{$\begin{array}{l}\text { Location of Contamination } \\
\text { Release Points }\end{array}$} & $\begin{array}{l}\text { The muckpile may contain small volumes of Resource Conservation and } \\
\text { Recovery Act-regulated constituents in addition to radiological constituents. }\end{array}$ \\
\hline & $\begin{array}{l}\text { The investigation results confirmed this and did not reveal any potential } \\
\text { releases off the muckpile. }\end{array}$ \\
\hline \multirow[t]{2}{*}{ Transport Mechanisms } & $\begin{array}{l}\text { Contamination may migrate through the muckpile into the native material as a } \\
\text { result of rainwater infiltration. }\end{array}$ \\
\hline & $\begin{array}{l}\text { Low levels of total petroleum hydrocarbons-diesel-range organics were found in } \\
\text { one native soil sample from under the muckpile at a depth of } 64 \text { feet, indicating } \\
\text { the oil was there before the muckpile was built over it. }\end{array}$ \\
\hline \multirow[t]{2}{*}{ Preferential Pathways } & Percolation of precipitation through the soils of the muckpile. \\
\hline & No preferred pathways were identified in the investigation. \\
\hline \multirow[t]{2}{*}{$\begin{array}{l}\text { Lateral and Vertical Extent of } \\
\text { Contamination }\end{array}$} & $\begin{array}{l}\text { Contamination could be locally significant, but vertical infiltration of } \\
\text { contaminants is probably limited to less than } 5 \text { feet. }\end{array}$ \\
\hline & $\begin{array}{l}\text { It is unclear whether the contamination found in the muckpile is the result of } \\
\text { infiltration or whether it was deposited with the muck during normal operations. } \\
\text { There were no indications that contamination migrated off the muckpile either } \\
\text { vertically or laterally. }\end{array}$ \\
\hline Groundwater Impacts & There are no groundwater impacts. \\
\hline \multirow[t]{2}{*}{ Future Land Use } & Nonresidential, zoned for nuclear and high explosives tests. \\
\hline & $\begin{array}{l}\text { The investigation results did not reveal any future land uses other than those } \\
\text { identified in the CSM. }\end{array}$ \\
\hline
\end{tabular}




\section{C.1.6 Results}

This section resolves the DQO decision for CAU 476. No decision rules were stated as "if/then" statements in the DQOs. However, the following "if/then" statements can be assumed from the DQOs.

\section{C.1.6.1 Decision Rules for CAU 476}

Decision Rule: If COPCs are above the PALs, then further action will be needed.

Result: After the site was characterized and a CADD prepared, DOE and NDEP came to an agreement that a risk-based approach could be used for characterizing the muckpiles. Following this decision, NDEP agreed that existing NDEP-approved DTRA muckpile CADDs could be re-evaluated using the risk-based approach. Using the risk-based approach, five biased samples had Cs-137 values that exceeded the PAL, and one random and three biased samples had TPH-DRO concentrations that exceeded PAL. However, of these, only one biased Cs-137 sample exceeded the FAL. Details are provided in Appendix D of this report.

Decision Rule: If laboratory results for the soil samples do not indicate the presence of COPCs above the PALs, then a CADD or CADD/CR will be prepared.

Result: The results of the investigation identified Cs-137 as a COC; therefore, a CADD/CR will be prepared that proposes a use restriction to control access to the site. 


\section{C.2.0 References}

DTRA, see Defense Threat Reduction Agency.

Defense Threat Reduction Agency. 2000. Corrective Action Investigation Plan for Corrective Action Unit 476: Area 12 T-Tunnel Muckpile, Nevada Test Site. Rev. 0. Las Vegas, NV.

EPA, see U.S. Environmental Protection Agency.

McArthur, R.D. and F.L. Miller, Jr. 1989. Off-Site Radiation Exposure Review Project, Phase II Soils Programs, DOE/NV/10384-23. Las Vegas, NV: Desert Research Institute.

NNSA/NV, see U.S. Department of Energy, National Nuclear Security Administration Nevada Operations Office.

U.S. Department of Energy, National Nuclear Security Administration, Nevada Operations Office. 2002. Industrial Sites Quality Assurance Project Plan, Nevada Test Site, Nevada, DOE/NV--372, Rev. 3. Las Vegas, NV.

U.S. Environmental Protection Agency. 1999. Test Methods for Evaluating Solid Waste, Physical/Chemical Methods, SW-846, $3^{\text {rd }}$ Edition. Washington, DC.

U.S. Environmental Protection Agency. 2004. Region 9 Preliminary Remediation Goals (PRGs). San Francisco, CA. 


\section{Appendix D \\ Risk Assessment for CAU 476}




\section{D.1.0 Risk-Based Corrective Action Process}

This section contains documentation of the ASTM Method E 1739-95 (ASTM, 1995) risk-based corrective action process as applied to CAU 476. The ASTM Method E 1739-95 defines three tiers or levels in evaluating DQO decisions involving increasingly more sophisticated analyses:

- Tier 1 - Sample results from source areas (highest concentrations) compared to the PALs based on generic (non-site-specific) conditions.

- Tier 2 - Sample results from exposure points compared to SSTLs calculated using site-specific inputs and Tier I formulas (from the ASTM procedure).

- Tier 3 - Sample results from exposure points compared to SSTLs and points of compliance calculated using chemical fate/transport and probabilistic modeling.

The risk based corrective action decision process stipulated in ASTM Method E 1739-95 is summarized in Figure D.1-1.

\section{D.1.1 Scenario}

Corrective Action Unit 476 (Area 12 T-Tunnel Muckpile) consists of one CAS:

- CAS 12-06-02, Muckpile

The T-Tunnel was mined into bedded ash flow tuff under Aqueduct Mesa. The tunnel was used for six nuclear tests between 1970 and 1987. The muckpile contains approximately 500,000 cubic yards of material consisting of mining debris (rock) generated during excavation of the tunnels and re-entry excavations following each test. Some nongeologic materials were disposed of in the muckpile during these excavations. These consisted of construction waste such as steel, wood, cables, grout, and possibly small amounts of RCRA-regulated constituents and radionuclides. One percent or less of the muckpile was expected to be composed of debris generated from re-entry operations (DNA, 1990). The muckpile was deposited on alluvium overlying the tuffs of the Calico Hills Formation. Groundwater is 800 to $900 \mathrm{ft}$ below ground surface (USGS/DOE, 2004). The muckpile was constructed out from the tunnel portal in an easterly direction. The muckpile is approximately $650 \mathrm{ft}$ across at its widest point and 1,000 ft long in an east-west direction. The thickness of the pad ranges from less than $1 \mathrm{ft}$ to approximately $70 \mathrm{ft}$. 


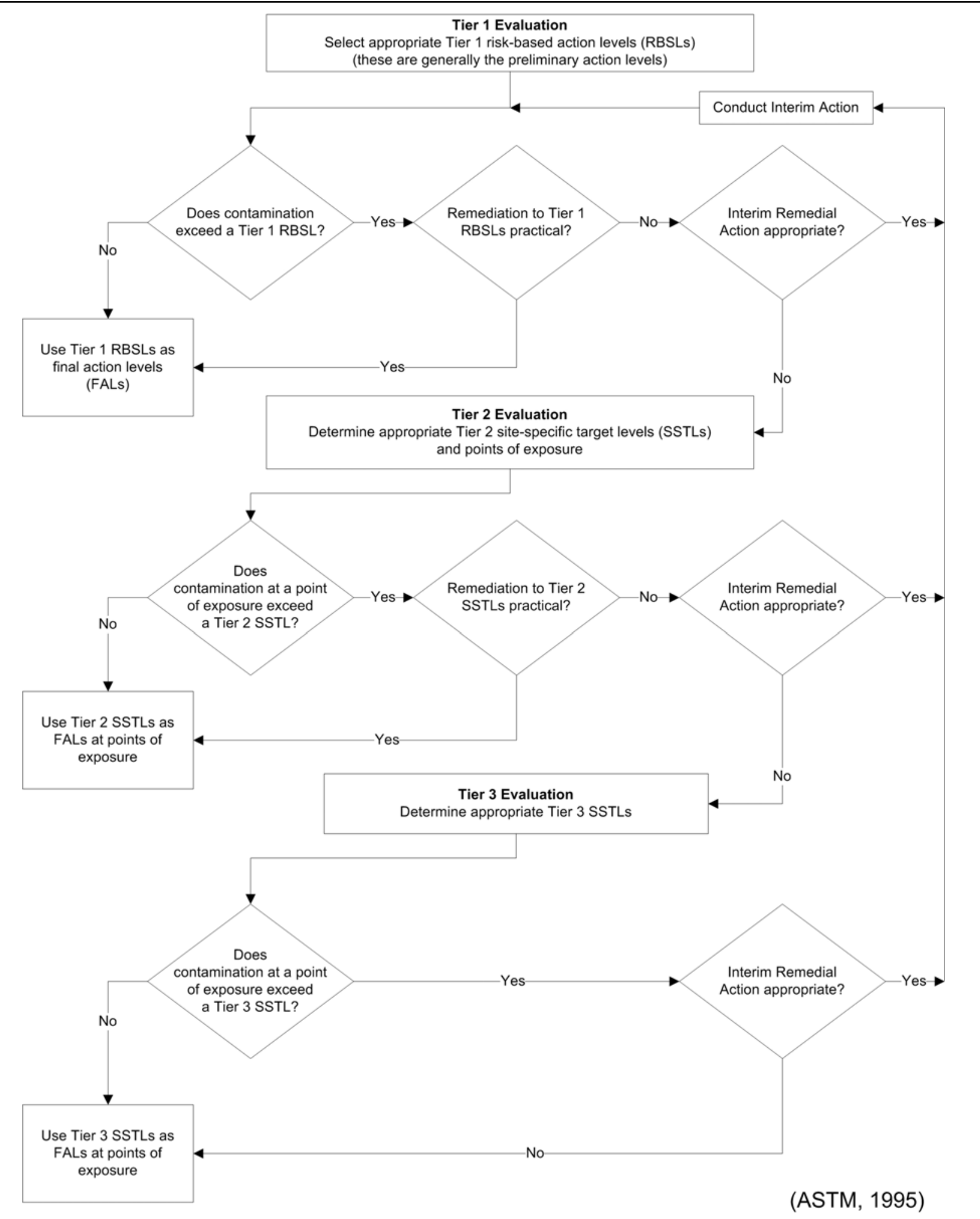

Figure D.1-1

ASTM Method E 1739-95 Risk-Based Corrective Action Decision Process 


\section{D.1.2 Site Assessment}

The CAI at CAU 476 involved soil sampling using rotosonic drilling techniques or hand tools. The investigation results identified TPH-DRO and Cs- 137 as COCs that exceeded the PALs as defined in the CAIP (DTRA, 2000). The maximum concentration of the COCs identified and their corresponding PALs (Tier 1 comparison) are presented in Tables D.1-1 (chemical results) and D.1-2 (radiological results).

Table D.1-1

Maximum Reported Chemical Values for Tier 1 Comparison

\begin{tabular}{|c|c|c|c|c|}
\hline Contaminant & CAS Number & Sample No. & $\begin{array}{c}\text { Result } \\
\text { (mg/kg) }\end{array}$ & $\begin{array}{c}\mathrm{PAL}^{\mathrm{a}} \\
(\mathrm{mg} / \mathrm{kg})\end{array}$ \\
\hline 1,1-Dichloroethene & $75-35-4$ & TS-14-24 & 0.0013 & 410 \\
\hline 1,2,3-Trichlorobenzene & $87-61-6$ & TS-20-20 & 0.0014 & 220 \\
\hline 1,2,4-Trimethylbenzene & $95-63-6$ & TS-13-14 & 0.001 & 170 \\
\hline 4-Methyl-2-Pentanone & $108-10-1$ & TS-18-3.0 & 0.0027 & 47,000 \\
\hline Acetone & $67-64-1$ & TS-S2-0.5 & 0.025 & 54,000 \\
\hline Arsenic & $7440-38-2$ & TS-13-14 & 13 & $23^{b}$ \\
\hline Barium & $7440-39-3$ & TS-S3-01 & 4,500 & 67,000 \\
\hline Benzoic Acid & $65-85-0$ & TS-18-20 & 0.27 & 100,000 \\
\hline Bis(2-Ethylhexyl)Phthalate & $117-81-7$ & TS-13-14 & 0.35 & 120 \\
\hline Cadmium & $7440-43-9$ & TS-09-30 & 0.4 & 450 \\
\hline Chromium & $7440-47-3$ & TS-S1-2.5 & 12 & 450 \\
\hline Diesel-Range Organics & $68334-30-5$ & TS-S7-01 & 1,200 & $100^{c}$ \\
\hline Di-N-Butyl Phthalate & $84-74-2$ & TS-S1-0.5 & 26 & 62,000 \\
\hline Lead & $7439-92-1$ & TS-13-14 & 210 & 800 \\
\hline Mercury & $7439-97-6$ & TS-12-14 & 0.079 & 310 \\
\hline Naphthalene & $91-20-3$ & TS-20-20 & 0.0016 & 190 \\
\hline N-Butylbenzene & $104-51-8$ & TS-20-20 & 0.00088 & 240 \\
\hline Selenium & $7782-49-2$ & TS-09-30 & 4.1 & 5,100 \\
\hline Silver & $7440-22-4$ & TS-09-30 & 2.4 & 5,100 \\
\hline Toluene & $108-88-3$ & TS-18-3.0 & 0.0009 & 520 \\
\hline Trichloroethene & $79-01-6$ & TS-12-14 & 0.00076 & 0.11 \\
\hline Trichlorotrifluoroethane & $76-13-1$ & TS-06-12 & 0.0011 & 77,900 \\
\hline
\end{tabular}

${ }^{\mathrm{a}} \mathrm{PAL}$ based on Region 9 Preliminary Remediation Goals (PRGs) (EPA, 2004).

${ }^{\mathrm{b}} \mathrm{Nevada}$ Test Site background plus two standard deviations.

${ }^{\mathrm{C} N e v a d a}$ Administrative Code 445A.2272 (NAC, 2003b)

Bold indicates the result exceeds the PAL.

CAS $=$ Chemical Abstracts Service

$\mathrm{mg} / \mathrm{kg}=$ Milligrams per kilogram

$\mathrm{PAL}=$ Preliminary action level 
Table D.1-2

Maximum Reported Radiological Values for Tier 1 Comparison

\begin{tabular}{|c|c|c|c|c|}
\hline Parameter & CAS Number & Sample No. & $\begin{array}{c}\text { Result } \\
\text { (pCi/g) }\end{array}$ & $\begin{array}{c}\text { PAL }^{\mathbf{a}} \\
\text { (pCi/g) }\end{array}$ \\
\hline \hline Actinium-228 & $14331-83-0$ & TS-03-09 & 3.92 & 15 \\
\hline Americium-241 & $14596-10-2$ & TS-04-63.5 & 0.62 & 12.7 \\
\hline Antimony-125 & $14234-35-6$ & TS-S1-0.5 & 3 & 18.1 \\
\hline Bismuth-212 & $14913-49-6$ & TS-04A-58 & 3.2 & 15 \\
\hline Bismuth-214 & $14733-03-0$ & TS-17-25 & 2.43 & 15 \\
\hline Cobalt-60 & $10198-40-0$ & TS-S6-0.5 & 1.76 & 2.7 \\
\hline Cesium-137 & $10045-97-3$ & TS-S6-0.5 & $\mathbf{3 8 2}$ & $\mathbf{1 2 . 2}$ \\
\hline Lead-212 & $15092-94-1$ & TS-02-8.5 & 3.54 & 15 \\
\hline Lead-214 & $15067-28-4$ & TS-17-25 & 2.87 & 15 \\
\hline Plutonium-238 & $13981-16-3$ & TS-S6-0.5 & 0.91 & 13 \\
\hline Plutonium-239 & $15117-48-3$ & TS-S6-0.5 & 2.87 & 12.7 \\
\hline Thallium-208 & $14913-50-9$ & QTS-05-24 & 1.15 & 15 \\
\hline
\end{tabular}

${ }^{a}$ PALs used as action levels. The PALs for radiological contaminants are based on background or the National Council on Radiation Protection and Measurements Report No. 129 recommended screening limits for construction, commercial, and industrial land-use scenario (NCRP, 1999) scaled from 25- to 15-millirem-per-year dose and the generic guidelines for residual concentration of radionuclides in DOE Order 5400.5 (DOE, 1993).

Bold indicates the result exceeds the PAL.

CAS $=$ Chemical Abstracts Service

$\mathrm{PAL}=$ Preliminary action level

$\mathrm{pCi} / \mathrm{g}=$ Picocuries per gram

\section{D.1.3 Site Classification and Initial Response Action}

The four major site classifications listed in Table 3 of the ASTM standard are: (1) immediate threat to human health, safety, and/or the environment; (2) short-term ( 0 to 2 years) threat to human health, safety, and/or the environment; (3) long-term (greater than 2 years) threat to human health, safety, and/or the environment; (4) no demonstrated long-term threats.

Based on the CAI, CAU 476 does not present an immediate threat to human health, safety, and/or the environment; therefore, no interim response actions are necessary at this site. The CAI demonstrated that the TPH-DRO contamination migrated into the muckpile at isolated areas and was possibly disposed of at the leading edge of the muckpile. The TPH-DRO results from one native soil sample had $47 \mathrm{mg} / \mathrm{kg}$ diesel. This sample was collected at a depth of $64 \mathrm{ft}$, and a nearby hole had a muck sample at a depth of $58 \mathrm{ft}$ that had $44 \mathrm{mg} / \mathrm{kg}$ TPH-DRO. No TPH-DRO was found in the muck above these samples. The TPH-DRO may have been released during construction of the muckpile and clean material deposited over it as the mining continued. The 
lack of TPH-DRO in the underlying native soil suggests that there is limited vertical migration. Cesium-137 was found at low levels in several biased surface samples and the background samples, but only one subsurface sample. This indicates that the Cs-137 may be the result of surface migration onto the muckpile because the surface area surrounding the muckpile was historically impacted with radionuclides. Based on this information, CAS 12-06-02 (Muckpile) is determined to be Classification 4 as defined by ASTM Method E 1739-95 (ASTM, 1995). At this site, COCs were identified; however, they do not pose long-term threats to human health and/or the environment.

\section{D.1.4 Development of Tier 1 Lookup Table of Risk-Based Screening Level Selection}

Tier 1 action levels have been defined as the PALs established during the DQO process. The PALs are a tabulation of chemical-specific (but not site-specific) screening levels based on the type of media (soil) and potential exposure scenarios (industrial). These are conservative estimates of risk, are preliminary in nature, and are used as action levels for site screening purposes. Although the PALs are not intended to be used as FALs, a FAL may be defined as the Tier 1 action level if individual constituent analytical results are below the corresponding Tier 1 action level. The FAL may also be established as the Tier I action level if individual constituent analytical results exceed the corresponding Tier 1 action level value and implementation of a corrective action based on the FAL is practical. The PALs are defined as:

- The EPA Region 9 Risk-Based PRGs for Industrial Soils (EPA, 2004). Note: The original PALs were the 2000 EPA Region 9 PRGs. Because the site is being re-evaluated, the newer PRGs were selected as the PALs.

- Background concentrations for RCRA metals will be evaluated when natural background exceeds the PAL, as is often the case with arsenic. Background is considered to be the mean plus two times the standard deviation of the mean based on data published in Mineral and Energy Resource Assessment of the Nellis Air Force Range (NBMG, 1998; Moore, 1999).

- Concentrations for TPH-DRO above $100 \mathrm{mg} / \mathrm{kg}$ per NAC 445A.2272 (NAC, 2003b).

- For COPCs without established PRGs, a protocol similar to EPA Region 9 will be used to establish an action level; otherwise, an established PRG from another EPA region may be chosen. 
- The PALs for radioactive contaminants are based on the National Council on Radiation Protection and Measurements (NCRP) Report No. 129 recommended screening limits for construction, commercial, and industrial land-use scenarios (NCRP, 1999) scaled to 25-mrem/yr dose constraint (Appenzeller-Wing, 2004) and the generic guidelines for residual concentration of radionuclides in DOE Order 5400.5 (DOE, 1993).

The PALs were developed based on an industrial scenario. Because CAU 476 in Area 12 is not assigned any work stations and is considered to be in a remote or occasional use area, the use of industrial land use based PALs is conservative. The Tier 1 lookup table is defined as the PAL concentrations or activities defined in the CAIP.

\section{D.1.5 Exposure Pathway Evaluation}

The DQOs stated that site workers would only be exposed to COCs through oral ingestion, inhalation, or dermal contact (absorption) due to exposure to potentially contaminated media (i.e., soil) at the CAU. The results of the CAI showed that all COCs identified in CAU 476 are localized near the release points and have not significantly migrated laterally or vertically in the subsurface. Because the contaminants were only identified in the soil of the T-Tunnel Muckpile, the only potential exposure pathway would be through worker contact with the contaminated soil. The lack of migration demonstrated by the analytical results, elapsed time since the suspected release, and the depth to groundwater supports the selection and evaluation of only the surface and shallow subsurface contact as the complete exposure pathway. Groundwater is not considered to be an exposure route or pathway.

\section{D.1.6 Comparison of Site Conditions with Tier 1 Risk-Based Screening Levels}

All analytical results for CAU 476 were less than corresponding Tier 1 action levels (i.e., PALs) except for those listed in Table D.1-3.

Table D.1-3

COPCs Detected Above Preliminary Action Levels

\begin{tabular}{|c|c|c|}
\hline & TPH-DRO & Cs-137 \\
\hline \hline CAS 12-06-02 Muckpile & $\mathrm{X}$ & $\mathrm{X}$ \\
\hline
\end{tabular}

COPC $=$ Contaminant of potential concern

$\mathrm{Cs}=$ Cesium

DRO = Diesel-range organics

$\mathrm{TPH}=$ Total petroleum hydrocarbons 


\section{D.1.7 Evaluation of Tier 1 Results}

For all constituents at CAU 476 not listed in Section D.1.6, the FALs were established as the Tier 1 risk-based screening levels. It was determined that no further action is required for these constituents at CAU 476.

It was determined by DTRA that remediation of the constituents listed in Table D.1-3 is not practical. Therefore, Tier 2 SSTLs were calculated for those constituents.

\section{D.1.8 Tier 1 Remedial Action Evaluation}

\section{$\underline{\text { TPH-DRO Evaluation }}$}

Remediation to Tier 1 action levels would be difficult and expensive while potentially not providing a significant risk reduction. Therefore, it was determined to assess the risk to human health posed by the hazardous constituents of TPH-DRO at CAU 476 under a Tier 2 evaluation before establishing FALs for TPH-DRO constituents or implementing a corrective action.

\section{$\underline{\text { Chemical Evaluation }}$}

None of the chemical constituents require remediation.

$\underline{\text { Radionuclide Evaluation }}$

Actions to remediate Cs-137 to the Tier 1 action level would be difficult and expensive while potentially not providing a significant risk reduction. Therefore, this radionuclide was moved to a Tier 2 evaluation before establishing a FAL.

\section{D.1.9 Tier 2 Evaluation}

No additional data were needed to complete a Tier 2 evaluation.

\section{D.1.10 Development of Tier 2 Table of SSTLS}

\section{Evaluation of TPH-DRO SSTLs}

The ASTM Method E 1739-95 (ASTM, 1995) stipulates that risk evaluations for TPH-DRO contamination be calculated and evaluated based on the risk posed by the potentially hazardous constituents of TPH-DRO. Section 6.4.3, "Use of Total Petroleum Hydrocarbon Measurements" 
of ASTM Method E 1739-95 states: "TPH-DRO should not be used for risk assessment because the general measure of TPH-DRO provides insufficient information about the amounts of individual chemical(s) of concern present" (see also Sections X1.5.4 and X1.42 of Method E 1739-95). Therefore, the individual potentially hazardous constituents in TPH-DRO were compared to corresponding Tier 2 SSTLs to evaluate the need for corrective action at CAU 476. Although Tier 2 SSTLs are generally calculated using site-specific inputs and general risk formulas, the Tier 2 SSTLs selected for the hazardous constituents of TPH-DRO are the EPA Region 9 PRGs (EPA, 2004). These SSTLs and the maximum reported level for each diesel constituent are presented in Table D.1-4.

Table D.1-4

Tier 2 SSTLs and CAU 476 Results for Hazardous Constituents of Diesel

\begin{tabular}{|c|c|c|c|}
\hline CAS No. & Common Name & $\begin{array}{l}\text { SSTL } \\
\text { (mg/kg) }\end{array}$ & $\begin{array}{l}\text { Maximum Reported } \\
\text { Value }(\mathrm{mg} / \mathrm{kg})\end{array}$ \\
\hline $108-67-8$ & 1,3,5-Trimethylbenzene & 70 & ND \\
\hline $91-57-6$ & 2-Methylnaphthalene ${ }^{a}$ & 190 & ND \\
\hline $56-55-3$ & Benzo(a)Anthracene & 2.1 & ND \\
\hline $71-43-2$ & Benzene & 1.4 & ND \\
\hline $50-32-8$ & Benzo(a)Pyrene & 0.21 & ND \\
\hline $100-41-4$ & Ethylbenzene & 400 & ND \\
\hline $91-20-3$ & Naphthalene & 190 & ND \\
\hline $108-88-3$ & Toluene & 520 & ND \\
\hline $1330-20-7$ & Total Xylene $^{\mathrm{b}}$ & 420 & ND \\
\hline $104-51-8$ & N-Butylbenzene & 240 & ND \\
\hline $103-65-1$ & N-Propylbenzene & 240 & ND \\
\hline $207-08-9$ & Benzo(k)Fluoranthene & 21 & ND \\
\hline $205-99-2$ & Benzo(b)Fluoranthene & 2.1 & ND \\
\hline $86-73-7$ & Fluorene & 26,000 & ND \\
\hline $85-01-8$ & Phenanthrene & 100,000 & ND \\
\hline $206-44-0$ & Fluoranthene & 22,000 & ND \\
\hline $129-00-0$ & Pyrene & 29,000 & ND \\
\hline $218-01-9$ & Chrysene & 210 & ND \\
\hline $120-12-7$ & Anthracene & 100,000 & ND \\
\hline $191-24-2$ & Benzo(g,h,i)Perylene & 29,000 & ND \\
\hline
\end{tabular}

${ }^{\mathrm{a} U}$ Uses PRG for naphthalene as surrogate

${ }^{\mathrm{b}}$ Total of $\mathrm{m}-$, o-, and $\mathrm{p}$-xylenes

CAS $=$ Chemical Abstracts Service $\mathrm{mg} / \mathrm{kg}=$ Milligrams per kilograms $\mathrm{ND}=$ Nondetect

$P R G=$ Preliminary remediation goal

SSTL $=$ Site-specific target level 


\section{Evaluation of Radiological Constituent SSTLs}

The Tier 2 evaluation consisted of evaluating the mixture of all radionuclides detected at the CAS to develop Tier 2 action levels for the radionuclides that exceeded Tier 1 levels. The CAS specific Tier 2 action levels were calculated using the RESRAD code (Yu et al., 2001) and site-specific parameters. The RESRAD calculations were based on continued use of the site under the occasional use area scenario, assuming that a site worker will be on site for 10 days per year, 8 hours a day for 5 years. A more detailed discussion of the RESRAD code, site-specific parameters used, and the printed RESRAD outputs are provided in Attachment A of this appendix. These SSTLs, the maximum reported level, and the average level for each radiological constituent are presented in Table D.1-5.

Table D.1-5

Tier 2 SSTLs and CAU 476 Results for Radiological Constituents

\begin{tabular}{|c|c|c|c|c|}
\hline CAS Number & Common Name & SSTL & Maximum Result & Average \\
\hline $10045-97-3$ & Cesium-137 & 374 & 382 & $20.4^{\mathrm{a}}$ \\
\hline
\end{tabular}

${ }^{\mathrm{a}}$ This is an average of muck and native random and biased samples

CAS $=$ Chemical Abstracts Service

$\mathrm{pCi} / \mathrm{g}=$ Picocuries per gram

SSTL $=$ Site-specific target level

Although all detected radionuclides at the CAS were used in the sum-of-fractions calculation, and a unique Tier 2 action level was developed for all radionuclides, only the radionuclide that initially exceeded Tier 1 level had a Tier 2 based FAL. The CAS specific FAL established for Cs-137 is the SSTLs listed in Table D.1-5.

\section{D.1.11 Comparison of Site Conditions with Tier 2 FALs}

Tier 2 action levels are typically compared to individual sample results from reasonable points of exposure (as opposed to the source areas as is done in Tier 1) on a point-by-point basis. Points of exposure are defined as those locations or areas at which an individual or population may come in contact with a COC originating from a CAS. For CAU 476, the Tier 2 action levels were compared to maximum constituent concentrations from each sample location and to the average concentration for the site.

A comparison of the maximum concentration of the hazardous constituents of TPH-DRO was conducted against the CAS-specific Tier 2 FALs as shown in Table D.1-4. All analytical results 
for hazardous constituents in TPH-DRO were nondetect for the muckpile (CAS 12-06-02). Therefore, TPH-DRO is not considered a COC at CAU 476.

None of the other organic or inorganic constituents exceed the occasional use area FALs for the muckpile (CAS 12-06-02).

A comparison between the maximum concentration of the radionuclides identified above Tier 1 action levels (Cs-137) was conducted against the CAS-specific Tier 2-based FAL (the Mixture Radionuclide Guidelines) listed in Attachment A of this appendix. For the muckpile (CAS 12-06-02), the maximum concentration from one of the biased samples exceeded the FAL; however, the average for the radionuclide is below the CAS-specific occasional use area FAL.

\section{D.1.12 Tier 2 Remedial Action Evaluation}

Based on the Tier 2 evaluation of the TPH-DRO hazardous constituents, the chemical constituents, and the radiological constituents, CAU 476 is not contaminated with organic or inorganic constituents under the industrial reuse scenario. The radiological constituent Cs-137 was identified at a concentration that would pose a risk to the industrial worker but not to the occasional use worker.

As all contaminant FALs were established as Tier 1 or Tier 2 action levels, a Tier 3 evaluation was considered unnecessary. 


\section{D.2.0 Regulatory Basis}

The FFACO Part III, Section III.3 (FFACO, 1996) stipulates conformance with Chapter 445A of the NAC (NAC, 2003a). Section NAC 445A.227 lists the factors to be considered in determining whether a corrective action is required.

Section NAC 445A.227 states:

1. Except as otherwise provided in NAC 445A.22715, the Director may require an owner or operator to take corrective action if the release of a hazardous substance, hazardous waste, or a regulated substance contaminates soil and the level of contamination exceeds the action level established for the soil pursuant to NAC 445A.2272.

2. In determining whether corrective action is required, the Director shall consider:

(a) The depth of any groundwater.

(b) The distance to irrigation wells or wells for drinking water.

(c) The type of soil that is contaminated.

(d) The annual precipitation.

(e) The type of waste or substance that was released.

(f) The extent of the contamination.

(g) The present and potential use for the land.

(h) The preferred routes of migration.

(i) The location of structures or impediments.

(j) The potential for a hazard related to fire, vapor, or explosion.

(k) Any other information specifically related to the site that the director determines is appropriate.

For a site where it is determined that corrective action is required (the corrective action process applies to all FFACO sites), Section NAC 445A.22705 (NAC, 2003c) stipulates a process to 
determine the necessary remediation standards (or FALs) based on an evaluation of the risk the site poses to public health and the environment.

Section NAC 445A.22705 states:

3. Except as otherwise provided in NAC 445A.22715, if an owner or operator is required to take corrective action pursuant to NAC 445A.227, the owner or operator may conduct an evaluation of the site, based on the risk it poses to public health and the environment, to determine the necessary remediation standards or to establish that corrective action is not necessary. Such an evaluation must be conducted using Method E 1739-95, adopted by the ASTM, as it exists on October 3, 1996, or an equivalent method approved by the Division.

4. The Division shall determine whether an evaluation complies with the requirements of Method E 1739-95, or an equivalent method of testing approved by the Division. The Division may reject, require revisions be made to, or withdraw its concurrence with the evaluation at any time after the completion of the evaluation for the following reasons:

(a) The evaluation does not comply with the applicable requirements for conducting the evaluation.

(b) Conditions at the site have changed.

(c) New information or previously unidentified information that would alter the results of the evaluation becomes available and demonstrates that the release may have a detrimental impact on public health or the environment.

Therefore, in compliance with Section NAC 445A.22705, DTRA conducted "an evaluation of the site, based on the risk it poses to public health and the environment, to determine the necessary remediation standards or to establish that corrective action is not necessary" using ASTM Method E 1739-95. 


\section{D.3.0 Recommendations}

Organic, inorganic, and radiological constituents detected in environmental samples during the CAI were evaluated against FALs to determine the nature and extent of COCs for CAU 476. Assessment of the data generated from the investigation activities indicates that the FAL was exceeded for Cs-137 in the muckpile. None of the other FALs for chemical or radiological constituents were exceeded, and Cs-137 is identified as the only COC present.

As COCs were identified above corresponding FALs, it was determined that closure in place with use restrictions is the best option for closing CAU 476. This is based on the fact that even though the FALs were exceeded, this remote, controlled access site poses only limited risk overall to public heath and the environment. Given the limited number of COCs (Cs-137 only), the negligible lateral and vertical migration, and the lack of potential impact to groundwater, it would create a greater hazard to worker safety, public health, and the environment to remove the contamination, transport it, and bury it at another location.

No further corrective action beyond establishing an administrative use restriction is necessary. 


\section{D.4.0 References}

ASTM, see American Society for Testing and Materials.

American Society for Testing and Materials. 1995. Standard Guide for Risk-Based Corrective Action Applied at Petroleum Release Sites, ASTM E 1739-95 (Reapproved 2002).

Philadelphia, PA.

Appenzeller-Wing, J., U.S. Department of Energy, National Nuclear Security Administration Nevada Site Office. 2004. Letter to T.A. Maize entitled, "Submittal of Proposed Radiological Preliminary Action Levels (PALs) for the Industrial Sites Project," 15 January. Las Vegas, NV.

DNA, see Defense Nuclear Agency.

DOE, see U.S. Department of Energy.

DTRA, see Defense Threat Reduction Agency.

Defense Threat Reduction Agency. 2000. Corrective Action Investigation Plan for Corrective Action Unit 476: Area 12 T-Tunnel Muckpile, Nevada Test Site, Rev. 0. Las Vegas, NV.

Defense Nuclear Agency. 1990. DNA Waste Management Document for DNA Activities at the Nevada Test Site, Las Vegas, NV: Test Directorate Nevada Operations Office.

EPA, see U.S. Environmental Protection Agency.

FFACO, see Federal Facility Agreement and Consent Order.

Federal Facility Agreement and Consent Order. 1996 (as amended). Agreed to by the State of Nevada, the U.S. Department of Energy, and the U.S. Department of Defense.

Las Vegas, NV.

Moore, J., Science Applications International Corporation. 1999. Memorandum to M. Todd (SAIC) entitled, "Background Concentrations for NTS and TTR Soil Samples," 3 February. Las Vegas, NV.

NAC, see Nevada Administrative Code.

NBMG, see Nevada Bureau of Mines and Geology.

NCRP, see National Council on Radiation Protection and Measurements.

National Council on Radiation Protection and Measurements. 1999. Recommended Screening Limits for Contaminated Surface Soil and Review of Factors Relevant to Site-Specific Studies, Report No. 129. Bethesda, MD. 
Nevada Administrative Code. 2003a. NAC 445A, "Water Controls.” Carson City, NV.

Nevada Administrative Code. 2003b. NAC 445A.2272, "Contamination of Soil: Establishment of Action Levels." Carson City, NV.

Nevada Administrative Code. 2003c. NAC 445A.22705, "Contamination of Soil: Evaluation of Site by Owner or Operator; Review of Evaluation by Division," Carson City, NV.

Nevada Bureau of Mines and Geology. 1998. Mineral and Energy Resource Assessment of the Nellis Air Force Range, Clark, Lincoln, and Nye Counties, Nevada, Open File Report 98-1. Reno, NV.

USGS/DOE, see U.S. Geological Survey and U.S. Department of Energy.

U.S. Department of Energy. 1993. Radiation Protection of the Public and the Environment. DOE Order 5400.5, Change 2. Washington, DC.

U.S. Environmental Protection Agency. 2004. Region 9 Preliminary Remediation Goals (PRGs). San Francisco, CA.

U.S. Geological Survey and U.S. Department of Energy. 2004. "USGS/DOE Nevada Water Wells." As accessed at http://nevada.usgs.gov/doe_nv/sitepage_temp.cfm?site_id= 371106116110401 on 8 March.

Yu C., A.J. Zielen, J.J Cheng, D.J. LePoire., E. Gnanapragasam, S. Kamboj, J. Arnish, A. Wallo III, W.A. Williams, and H. Peterson. 2001. User's Manual for RESRAD Version 6, ANL/EAD-4, Argonne National Laboratory, Environmental Assessment Division, Argonne, IL. 


\section{Attachment A}

Derivation of Residual Radioactive Material Guidelines for Radionuclides in Soil at Corrective Action Unit (CAU) 476, Area 12 T-Tunnel Muckpile, Nevada Test Site, Nevada 
Derivation of Residual Radioactive Material Guidelines for Radionuclides in Soil at Corrective Action Unit (CAU) 476, Area 12 T-Tunnel Muckpile, Nevada Test Site, Nevada

\author{
March 2007
}

\author{
Prepared by: \\ Stoller-Navarro Joint Venture, \\ 7710 W. Cheyenne, Las Vegas, Nevada 89129
}

Work sponsored by United States Department of Energy, National Nuclear Security Administration Nevada Site Office, Environmental Restoration Division, Las Vegas, Nevada 


\section{Derivation of Residual Radioactive Material Guidelines for Radionuclides in Soil at Corrective Action Unit (CAU) 476, Area 12 T-Tunnel Muckpile, Nevada Test Site, Nevada}

\subsection{Introduction}

The U.S. Department of Energy (DOE), the U.S. Department of Defense (DoD) (through the Defense Threat Reduction Agency [DTRA]), and the National Nuclear Security Administration Nevada Site Office (NNSA/NSO) Environmental Restoration Division have numerous sites impacted from the development, testing, and production of nuclear weapons. These impacts can take the form of chemical and/or radiological contaminants. Similar to its approach for chemical contamination, DoD and NNSA/NSO are committed to properly evaluating, radiologically characterizing and, where appropriate, remediating these sites to ensure the doses to radiation workers and members of the public are maintained as-low-as-reasonably achievable (ALARA), at a minimum, below the primary dose limits as stated in DOE Order 5400.5 (DOE, 1993).

To accomplish this, the potential for residual radioactive contamination in soils must be evaluated to determine the status of compliance with the requirements of DOE Order 5400.5 (DOE, 1993). The DOE Order 5400.5 requires that: "The Authorized Limits shall be established to (1) provide that, at a minimum, the basic dose limits ... will not be exceeded, or (2) be consistent with applicable generic guidelines." Because generic guidelines have not been established for volumetric residual radioactivity for the radionuclides of concern at CAU 476, Authorized Limits or final action levels (FALs) were derived using the Residual Radioactive (RESRAD) model and computer code (Yu et al., 2001). The goal of this effort was to produce Authorized Limits, in units of picocuries per gram ( $\mathrm{pCi} / \mathrm{g})$ in soil above background, for CAU 476 that would result in radiation doses less than $25 \mathrm{mrem}$ per year (mrem/yr) to an industrial worker at the site.

To develop the FALs, a "realistic" yet conservative radiation dose analysis was conducted using approved exposure scenarios and site-specific data to determine the translation between surface soil concentrations and individual radiation doses. For this analysis, site-specific data included soil sampling results obtained during site investigation activities at CAU 476, and meteorological data obtained from the Air Resources Laboratory (ARL)/Special Operations and Research Division (SORD). This report provides the radiation dose modeling analysis supporting the technical derivation of the Authorized Limits for CAU 476, Area 12 T-Tunnel Muckpile, Nevada Test Site (NTS), Nevada. This report also defines the radionuclides considered and approved exposure scenarios for the NTS, identifies the applicable exposure pathways and key input data or assumptions, presents the radiation doses for unit concentrations of radionuclides in soil, and establishes the FALs for CAU 476. Figure 1-1 shows the location of CAU 476 at the NTS. 


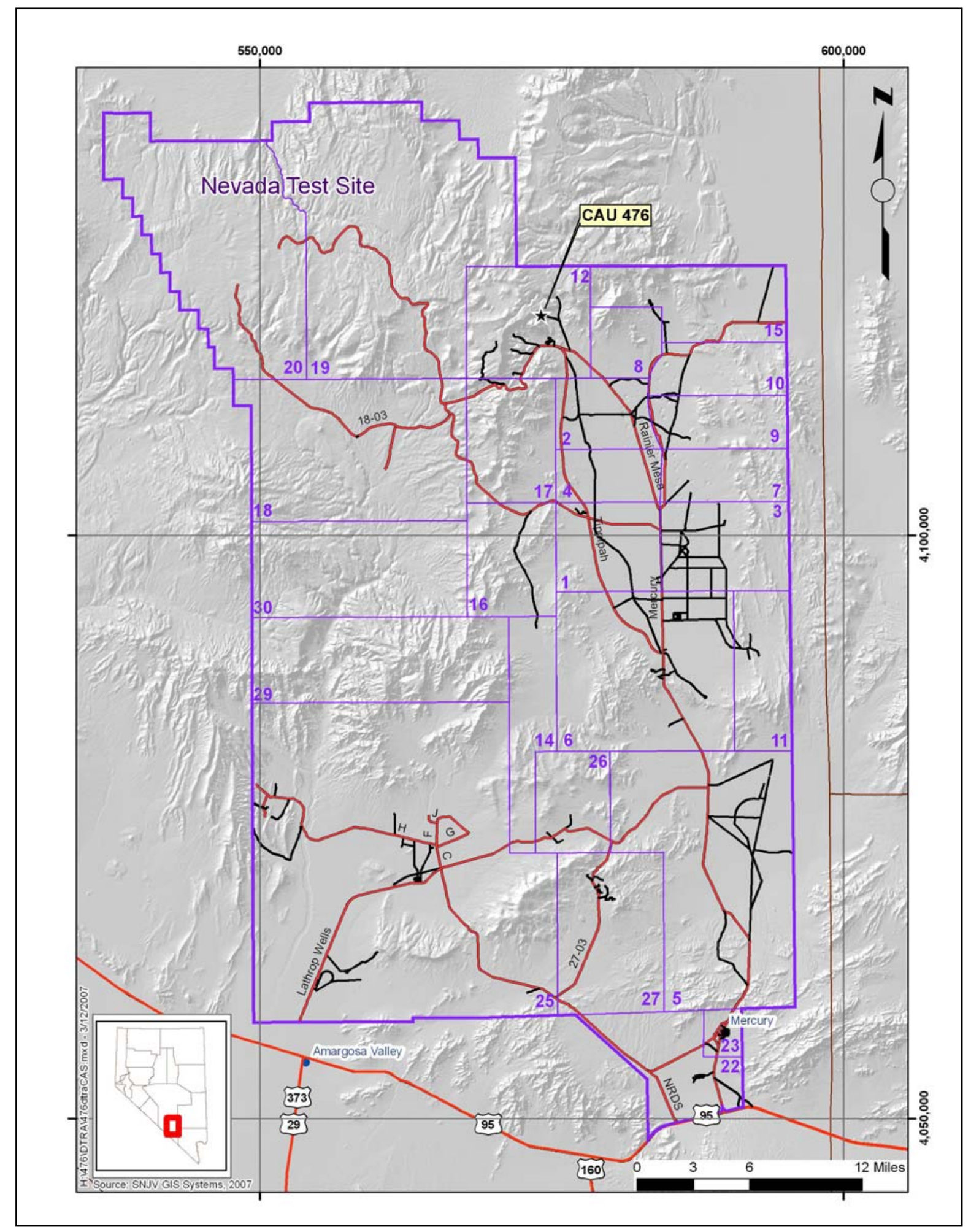

Figure 1-1

Nevada Test Site Map Showing CAU 476 Location 


\subsection{Facility Description}

Corrective Action Unit 476 comprises one CAS, the T-Tunnel Muckpile. The T-Tunnel Muckpile consists primary of mining debris (rock) generated during tunnel excavation and construction in support of weapons effect testing. Eight tests were conducted at T-Tunnel (DTRA, 2000) including six nuclear tests from 1970 to 1987 and two high explosive tests in 1997. A site plan and depositional chronology for the muckpile is illustrated in the CAIP. Only limited information is available about the muckpile. Specifics concerning potential wastes buried within or released upon the muckpile have not been documented due to unregulated disposal activities associated with muckpile operations.

The T-Tunnel, constructed beneath Aqueduct Mesa, was mined into bedded, ash-flow tuff from 800 to $1,100 \mathrm{ft}$ below the mesa surface. The surface elevation at the tunnel portal is about $5,600 \mathrm{ft}$ above mean sea level. The T-Tunnel was excavated in a westerly direction into Aqueduct Mesa from the side of a canyon. The muckpile was built out in an easterly direction from the tunnel portal, partially filling an eastward draining canyon. The muckpile generally widens and thickens away from the tunnel, terminating approximately 1,000 ft east of the tunnel portal. The muckpile is approximately $650 \mathrm{ft}$ across at its widest point, which occurs about $500 \mathrm{ft}$ from the tunnel portal, and is roughly $70 \mathrm{ft}$ thick near its eastern edge.

\subsection{Operational History}

The T-Tunnel was operated intermittently between 1968 and 1997 during the period of nuclear and non-nuclear weapons effects testing. The tunnel was designed to contain the pressures and temperatures created during nuclear tests. Re-entry to the tunnel after a test typically involved additional horizontal mining and the generation of rock debris and construction wastes such as wood, cabling, scrap metal, and cementitious mixtures, which could have contain low levels of radioactivity. The material excavated during re-entry were generally hauled outside the tunnel to the edge of the existing muckpile, dumped off the edge, and then leveled. Six nuclear tests were conducted in T-Tunnel. Certain portions of the muckpile can be ascribed to each of these tests.

\subsection{Release Information}

There have been no known or suspected releases from CAS 12-06-02. Venting was documented from a J-Tunnel test, Des Moines, on June 13, 1962, in the area that is approximately 1,300 $\mathrm{ft}$ directly east of the muckpile (DOE/NV, 1996). 


\subsection{Site Investigation Activities}

In December 1999 and January 2000, seismic and surface geophysical surveys were performed at the T-Tunnel Muckpile to determine the approximate thickness of the muckpile and to locate compressed gas cylinders that were reported to have been buried in the muckpile. Results of these surveys were used to guide the placement of the boreholes so that to minimized the chance of encountering the cylinders when drilling.

\subsection{Site Investigation Plans}

Corrective action investigation activities were performed as set forth in the CAU 476 CAIP (DTRA, 2000) from April 10 to May 2, 2000. The objectives of the CAU 476 CAIP were to address the decision statements in the project-specific Data Quality Objectives (DQOs) by:

- Characterizing the surface and near-surface ( 0 to $5 \mathrm{ft}$ ) muckpile material for contaminants of potential concern (COPC).

- Characterizing the muckpile subsurface (greater than $5 \mathrm{ft}$ ) for COPCs.

- Characterizing the native soils (from 2 to $5 \mathrm{ft}$ below the bottom of the muckpile) for impacts from COPCs.

- Establishing background levels of radioactivity and meals for the native soils surrounding the muckpile.

The DQO process is a seven-step strategic planning approach based upon the scientific method used to plan data collection activities for CAU 476, Area 12 T-Tunnel Muckpile, NTS. The DQOs are designed to ensure that data collected will provide sufficient and reliable information to identify, evaluate and technically defend the recommended corrective actions (i.e., no further action, closure in place, or clean closure).

The primary objective of the investigation was to provide sufficient information and data to develop appropriate corrective action alternatives for CAU 476. This objective was achieved by identifying the nature and extent, both horizontal and vertical of COCs (i.e., COPCs at concentrations above action levels).

The investigation strategy was developed by representatives of NDEP and DTRA, in accordance with U.S. Environmental Protection Agency (EPA) Guidance for the Data Quality Objectives Process, EPA QA/G-4 (EPA, 1994). The CAU 476 CAIP contains a description of the investigation strategy and the DQO process.

\subsection{Summary of Specific Site Investigation Activities}

This section provides a brief description of work activities conducted to support the investigation of radioactive contamination at CAU 476. 
From April 10 through May 2, 2000, CAI activities were performed at the T-Tunnel Muckpile as set forth in the CAIP (DTRA, 2000). The purpose of the CAI was to determine the presence and extent of COPCs within or beneath the muckpile, and to provide sufficient information and data to develop appropriate corrective action strategies for the muckpile. As outlined in the CAIP, the following tasks were performed:

- Background sampling - Three locations were identified and excavated by hand to a depth of approximately 18 inches to collect background native soil samples. Samples were field screened and submitted for radionuclide analysis.

- Surface sampling - Eight locations were selected to characterize the surface/shallow subsurface of the muckpile. Seven boleholes were drilled to a depth of $5 \mathrm{ft}$, and a continuous core was extracted from each borehole. Each core was field screened, and the portion(s) of the core with the highest result was collected for laboratory analysis. If no portion of the core exhibited elevated results, the interval between 0.5 and $1.5 \mathrm{ft}$ was colleted. Nine surface/shallow subsurface samples were collected from these locations and submitted for analysis.

- Muckpile content/underlying soils sampling - Twenty boreholes were drilled to characterize the subsurface of the muckpile from $5 \mathrm{ft}$ below ground surface to 2 to $5 \mathrm{ft}$ into the native soil under the muckpile. A continuous core was extracted from each borehole and, field screened, and 42 samples were submitted for analysis. One sample was taken at the bottom of each borehole to represent the native materials beneath the muckpile.

Field screening of the core recovered from the boreholes provide guidance for collection of two additional samples. Field screening results also provide additional characterization information on which to base corrective action decisions. 


\subsection{Site Investigation Sample Results}

The RESRAD calculations are based on validated analytical soil sample results obtained during site investigation activities and other applicable information specified in the CAIP. The RESRAD calculations are based upon the value of the maximum radionuclide concentration. The RESRAD calculations of the area were performed for the contaminants of concern (COC) present in the CAU 476 muckpile using the maximum radionuclide concentrations obtained from the CAI soil sample results. Appendix A of the CAU 476 CADD contains a detailed description of the sample results, analytical parameters, and laboratory methods used to analyze the soil samples.

The maximum principal radionuclide concentrations (including background) detected at the CAU 476 are listed in Table 4-1.

Table 4-1

CAU 476 Principal Radionuclide Concentration Found in Soil Samples

\begin{tabular}{|c|c|c|c||}
\hline Sample Number & Sample Depth (ft bgs) & Radionuclide & pCi/g \\
\hline \hline TS-B2-01 & $0.5-1.5$ & Americium-241 & 3.8 \\
\hline TS-S1-0.5 & $0-1$ & Antimony-125 & 382 \\
\hline TS-S6-0.5 & $0-1$ & Cesium-137 & 1.76 \\
\hline TS-S6-0.5 & $0-1$ & Cobalt-60 & 1.03 \\
\hline TS-B2-01 & $0.5-1.5$ & Plutonium-238 & 9.60 \\
\hline TS-B2-02 & $0.5-1.5$ & Plutonium-239 & 13.0 \\
\hline TS-S6-0.5 & $0-1$ & Strontium-90 & \\
\hline
\end{tabular}

$\mathrm{ft}=$ Feet below ground surface

$\mathrm{pCi} / \mathrm{g}=$ Picocuries per gram 


\subsection{Initial Concentrations for Principal Radionuclides}

Principal radionuclides are defined as radionuclides with a half-life greater than six months. The decay products of any principal radionuclide down to, but not including, the next principal radionuclide in its decay chain are defined as associated radionuclides. RESRAD assumes that a principal radionuclide is in secular equilibrium with its associated radionuclides at the point of exposure. Therefore, associated radionuclides and radionuclides with half-lives less than six months are not input into the RESRAD calculations.

\subsection{Authorized Values for Initial Concentrations of Principal Radionuclides}

The authorized exposure scenarios specify that value of the arithmetic mean plus the 95 percent upper confidence limit (UCL) obtained from site-specific sampling results be entered as the principal radionuclide concentrations for RESRAD calculates. The sample results for all samples with radionuclide concentrations above the MDC within the land parcels are entered into the EPA software application ProUCL version 3.0. The ProUCL software is used to calculate the 95 percent UCL for principal radionuclide concentrations based on the distribution of the unknown mean.

For instances where the ProUCL software determined that there were not enough data to calculate the 95 percent UCL for a specific radionuclide, the maximum concentration from the sample dataset was used as the initial concentration for that radionuclide.

\subsection{Authorized Values Initial Concentrations of Principal Radionuclides for Area Averaging/Location Specific Scenarios}

The DOE Order 5400.5 (DOE, 1993) states: "Residual concentrations of radioactive material in soil are defined as those in excess of background concentrations averaged over an area of $100 \mathrm{~m}^{2}$ " (5400.5, IV, 4.a.). DOE Order 5400.5 also states: "If the average concentration of any surface or below-surface area less than or equal to $25 \mathrm{~m}^{2}$, exceeds the limit or guideline by a factor of $(100 / \mathrm{A})^{0.5}$, [where $\mathrm{A}$ is the area (in square meters) of the region in which concentrations are elevated], limits for "hot-spots" shall also be developed and applied" (5400.5, IV, 4.a.(1)). DOE Order 5400.5, IV, 4.a.(1) indicates that criterion for these location-specific analysis is discussed in DOE G 441.1-XX (DOE 2002) Section 5.2.2.

The purpose of the location-specific analysis criterion is to ensure that applying the homogeneous criteria, in which the concentrations of residual radioactive material are averaged over a 100-square meter $\left(\mathrm{m}^{2}\right)$ area, does not result in the release of small areas that, because of averaging, contain unacceptably high concentrations of residual radioactive material. The location-specific criterion is used to supplement Authorized Limits for larger areas and is intended to prevent excessive exposures from a small, contaminated area that is within a larger area that meets the basic Authorized Limits. Thus, it is intended for use in areas where the residual radioactive material concentrations are not uniform. Also, the above criterion was derived conservatively, assuming the Authorized Limits were based on a dose constraint of $25 \mathrm{mrem} / \mathrm{yr}$ and selected to ensure unlikely exposure conditions would not cause the primary dose limit (100 mrem/yr) to be exceeded. The authorized exposure scenarios specify that the value of the maximum concentration of principal radionuclides obtained from site-specific 
sampling results be entered as the principal radionuclide concentrations for RESRAD location-specific calculations. The authorized area parameters for RESRAD location-specific calculations are $1 \mathrm{~m}^{2}, 10 \mathrm{~m}^{2}$, and $100 \mathrm{~m}^{2}$ contamination areas.

\subsection{Inhomogeneous Contamination and Initial Radionuclide Concentrations}

A contaminated zone is inhomogeneous if it contains a contaminated region within which the concentration of a radionuclide exceeds three times the average for the contaminated zone. RESRAD uses a mathematical construct that assumes uniform distribution of radionuclides within a volume. However, RESRAD recognizes that radiological contamination is inhomogeneous in nature and provides detailed guidance for applying inhomogeneous criteria (e.g., location-specific criteria, sum of fractions rule). The RESRAD User's Manual states that the inhomogeneous release criteria are generally more realistic and hence less restrictive than the homogeneous release criteria (Yu, et.al, 2001). This shows that the approved initial radionuclide concentration values (i.e., arithmetic mean plus 95 percent UCL or the maximum radionuclide concentration from the sample dataset) will result in more restrictive release criteria. The arithmetic mean plus the 95 percent UCL are used for the initial concentrations of principal radionuclides when the sample results are obtained using a random sampling method. The maximum radionuclide concentration values are used for the initial concentrations of principal radionuclides when the sample results are obtained using a non-random (e.g., bias or judgmental sampling) sampling method.

RESRAD states that a statistical approach should always be considered as a first priority regarding the estimation of soil concentrations, as cited in the Data Collection Handbook to Support Modeling Impacts of Radioactive Material in Soil (Yu et al., 1993). The 95 percent UCL represents a value that has a 5 percent chance that the actual mean of the dataset would exceed it. The 95 percent UCL is computed using the EPA code ProUCL. The code calculates the 95 percent UCL based on the distribution of the dataset (e.g., normal, log-normal, gamma, non-parametric).

The ProUCL software has been developed to compute an appropriate 95 percent UCL of the unknown population mean to support exposure assessment and cleanup decisions for EPA projects. A 95 percent UCL of the unknown population arithmetic mean is often used to:

- Estimate the exposure point concentration term,

- Determine the attainment of cleanup standards,

- Estimate background level mean contaminant concentrations, or

- Compare the soil concentrations with site-specific soil screening levels.

It is important to compute a reliable, conservative, and stable 95 percent UCL of the population mean using the available data. The 95 percent UCL should approximately provide the 95 percent coverage for the unknown population mean.

The EPA has recommended that the maximum value of the dataset be used for the initial EPC term when the 95 percent UCL exceeds the maximum (EPA, 1992). However, if the maximum value of the dataset is used, then most of the statistical data associated with the distribution of the dataset are ignored (except for the maximum). Therefore, by using the mean plus the 95 percent 
UCL the statistical data associated with the dataset are retained, and the value approaches or exceeds the maximum value of the dataset as recommended by EPA.

\subsection{Initial Concentrations of Principal Radionuclide for CAU 476}

The initial radionuclide concentrations used for the RESRAD calculations are those listed in Table 4-1. These maximum radionuclide concentration values were used to perform the RESRAD calculations to demonstrate conservatism. 


\subsection{Authorized RESRAD Exposure Pathways and Scenarios}

This section describes the input parameters, exposures scenarios, and guidance for calculating site-specific radiological remediation levels for projects using the RESRAD computer code, as agreed to by NNSA/NSO, Stoller-Navarro Joint Venture (SNJV), the NTS M\&O Contractor, and NDEP.

\subsection{Guidance for RESRAD Calculations}

The guidance in this section was developed by NNSA/NSO, SNJV, the M\&O Contractor, and NDEP and is only applicable to soils containing residual radioactive material. This guidance does not apply to structures, facilities, equipment, and building materials containing contaminated surfaces or volume contamination. The primary dose limit for any member of the public is 100-millirem (mrem) total effective dose equivalent (TEDE) in a year. This limit applies to the sum of internal and external doses resulting from all modes of exposure to all radiation sources other than background radiation and doses received as a patient from medical sources as required by DOE 5400.5, II.1.a.(3)(a) (DOE, 1993). The dose constraint is defined as one quarter of the dose limit (i.e., 25-mrem) and will be applied to ensure that in a 1,000-year period the maximally exposed individual does not exceed the dose constraint in any single year. The requirements of Chapter IV of DOE 5400.5 Chapter IV will not specifically apply if NNSA/NSO chooses to continue to own and actively control access or use of the site. However, the radiation protection requirements in the other sections of DOE 5400.5 will apply to NNSA/NSO owned and maintained sites.

Due to the large spatial variability in background amongst sites, the "above background criterion" will be defined as the concentration of a specific radionuclide in soil that equals or exceeds its corresponding PAL. The source data for these radionuclide specific PALs are taken directly from NCRP Report No. 129 Table 2.1, Construction, Commercial, Industrial land-use scenario column for a 25-mrem dose constraint (NCRP, 1999). The generic guidelines for residual concentrations of radium ( $\mathrm{Ra}$-226, Ra-228, thorium (Th)-230, and Th-232 are found in Chapter IV of DOE Order 5400.5, Change 2, Radiation Protection of the Public and Environment (DOE, 1993).

Background radiation refers to the local area and includes:

- Concentration of naturally occurring radionuclides.

- Cosmic radiation.

- Radionuclides of anthropogenic origin that have been globally dispersed and are present at low concentrations such as fallout from nuclear weapons. (Note: This is not the case at the NTS because the historical aspects of the NTS [e.g., above- and below-ground testing, and other operations resulted in dispersion of radionuclides locally].)

Due to the impracticality of determining "true" background, a dose constraint with no background subtraction will be used (i.e., a dose constraint not in excess of background). The 
use of the dose constraint with no background subtraction is a far more conservative and sensitive approach because it does not deal with the uncertainty of natural background.

\subsection{Description of Approved Scenarios}

Based on the future land use as identified in the Nevada Test Site Resource Management Plan (DOE/NV, 1998), the following two exposure scenarios have been identified as "actual" and "likely" use scenarios. Stoller-Navarro Joint Venture has approval to use two scenarios (Scenario A and Scenario B) for use with the RESRAD code (NDEP, 2004). Both scenarios consider radiation exposures to the critical population group via the following pathways:

- Direct exposure to external radiation from the contaminated soil.

- Internal dose from inhalation of airborne radionuclides.

- Internal dose from ingestion of contaminated soil.

The two scenarios vary the parameters associated with the future land use of the site but use the same dose constraint parameter of $25 \mathrm{mrem} / \mathrm{yr}$. Scenario A is approved for sites in Mercury or within $500 \mathrm{ft}$ of an active building. Scenario B is approved for all other sites. Scenarios A and B are briefly described below.

For Scenario A, the future land use assumes continued industrial use of the site. This scenario addresses long-term exposure received by industrial workers exposed daily to residual levels of radionuclides in soil during an average workday outdoors on site (EPA, 1991). Scenario A parameters are based on the following:

- A worker will be outdoors at the site for a total of 2,000 hr per year (hr/yr) (250 days per year, 8 hours per day) for a duration of 25 years.

- Indoor fraction time is zero, which means that the worker is outside being exposed for the entire workday.

- The outdoor time fraction is 0.228 and is calculated by dividing the total work hours at the site per year $(2,000 \mathrm{hr} / \mathrm{yr})$ by the total number of hours in a year $(8,760 \mathrm{hr} / \mathrm{yr})$.

- Worker exposures are limited to working hours and do not include contributions from ingestion of drinking water, plant foods, meat, or fish taken from the immediate area.

For Scenario B, the future land use assumes land use restrictions with a low occupancy factor and lighter work activities at the site. The assumptions for Scenario B include the following:

- A worker will be at the site and outdoors for a total of $335 \mathrm{hr} / \mathrm{yr}$ for a duration of 25 years.

- The indoor fraction time is zero

- The outdoor time fraction is 0.038 , which is calculated by dividing the total work hours at the site per year $(335 \mathrm{hr} / \mathrm{yr})$ by the total number of hours in a year $(8,760 \mathrm{hr} / \mathrm{yr})$. 
- The worker exposures are limited to working hours and do not include contributions from ingestion of drinking water, plant foods, meat or fish taken from the immediate area.

When Scenario B is selected, a Use Restriction will be included at closure that will state the use scenario and the requirement for an occupant agency or entity to re-evaluate the closure if site use changes to fit the parameters of Scenario A.

Table 6-1 lists the pathways considered for Scenarios A and B.

Table 6-1

Summary of Pathways Considered for Scenarios A and B

\begin{tabular}{|c|c|c|}
\hline Pathway & Scenario A & Scenario B \\
\hline \hline External exposure & Yes & Yes \\
\hline Particulate inhalation & Yes & No \\
\hline Radon inhalation & No & No \\
\hline Ingestion of soil & Yes & No \\
\hline Ingestion of produce from on-site garden & No & No \\
\hline Ingestion of meat from on-site livestock & No & No \\
\hline Ingestion of milk from on-site livestock & No & No \\
\hline Ingestion of fish from on-site pond & No & No \\
\hline Ingestion of water from on-site well & & Nom
\end{tabular}

\subsection{RESRAD Parameters}

The RESRAD User's Manual states that: "The RESRAD default parameter values were carefully selected and are realistic, although conservative, parameter values. (In most cases, use of these values will not result in underestimation of the dose or risk.) Site-specific parameters should be used whenever possible. Therefore, use of default values that significantly overestimate the dose or risk for a particular site is discouraged" (Yu et al., 2001).

Table 6-2 lists all of the RESRAD default values along with the site-specific RESRAD parameters approved for use with Scenarios A and B. A reference or reason is provided for parameters that require site-specific input. 
Table 6-2

Approved RESRAD Parameters

(Page 1 of 6 )

\begin{tabular}{|c|c|c|c|c|c|}
\hline Parameter & Units & Scenario A & Scenario B & Defaults & Reference/Rationale \\
\hline Dose Conversion Factors & & & & & Use FGR 13 Morbidity \\
\hline \multicolumn{6}{|l|}{ R02 Exposure Pathways } \\
\hline Pathway 1- External Gamma & & Active & Active & & \\
\hline Pathway 2- Inhalation & & Active & Active & & \\
\hline Pathway 3- Plant Ingestion & & Suppressed & Suppressed & & \\
\hline Pathway 4- Meat Ingestion & & Suppressed & Suppressed & & \\
\hline Pathway 5- Milk Ingestion & & Suppressed & Suppressed & & \\
\hline Pathway 6- Aquatic Foods & & Suppressed & Suppressed & & \\
\hline Pathway 7- Drinking Water & & Suppressed & Suppressed & & \\
\hline Pathway 8- Soil Ingestion & & Active & Active & & \\
\hline Pathway 9- Radon & & Suppressed & Suppressed & & \\
\hline \multicolumn{6}{|l|}{ R011 Contaminated Zone (CZ) } \\
\hline Area of CZ & $\mathrm{m}^{2}$ & Site Specific & Site Specific & $1.000 \mathrm{E}+04$ & $\begin{array}{l}\text { Maximum area of contamination out to two successive } \\
\text { sample intervals below PALs. ( 15 ft intervals } \\
\text { laterally) }\end{array}$ \\
\hline Thickness of CZ & $\mathrm{m}$ & Site Specific & Site Specific & $2.000 \mathrm{E}+00$ & $\begin{array}{l}\text { Maximum identified depth plus two successive intervals } \\
\text { below PALs as identified during the site } \\
\text { characterization. }(\sim 5 \mathrm{ft} \text { intervals vertically })\end{array}$ \\
\hline Length Parallel to Aquifer Flow & $\mathrm{m}$ & not used & not used & $1.000 \mathrm{E}+02$ & Not used with the above pathway selection \\
\hline Radiation Dose Limit & $\mathrm{mrem} / \mathrm{yr}$ & 25 & 25 & $2.5 \mathrm{E}+001$ & RESRAD Default (DOE, 1993) \\
\hline Elapsed Time Since Placement of Material & $\mathrm{yr}$ & 0.0 & 0.0 & 0.0 & RESRAD Default \\
\hline \multicolumn{6}{|l|}{ R012 Initial Principal Radionuclide } \\
\hline $\begin{array}{l}\text { Site-Specific Parent Radionuclide with half- } \\
\text { life greater than } 180 \text { days, does not include } \\
\text { naturally occurring and primordial } \\
\text { radionuclides }\end{array}$ & $\mathrm{pCi} / \mathrm{g}$ & Site Specific & Site Specific & 0.0 & The arithmetic mean plus the $95 \%$ UCL for the site. \\
\hline
\end{tabular}


Table 6-2

Approved RESRAD Parameters

(Page 2 of 6 )

\begin{tabular}{|c|c|c|c|c|c|}
\hline Parameter & Units & Scenario A & Scenario B & Defaults & Reference/Rationale \\
\hline \multicolumn{6}{|c|}{ R013 Cover and Contaminated Zone Hydrological Data } \\
\hline Cover Depth & $\mathrm{m}$ & $\begin{array}{l}\text { Site } \\
\text { Specific }\end{array}$ & $\begin{array}{l}\text { Site } \\
\text { Specific }\end{array}$ & 0.0 & $\begin{array}{l}\text { The minimum depth as identified during the site } \\
\text { characterization }\end{array}$ \\
\hline Density of Cover Material & $\mathrm{g} / \mathrm{cm}^{3}$ & 1.5 & 1.5 & 1.5 & RESRAD Default unless site data significantly different \\
\hline Cover Depth Erosion Rate & $\mathrm{m} / \mathrm{yr}$ & 1.000E-03 & $1.000 \mathrm{E}-03$ & 1.000E-03 & RESRAD Default unless site data significantly different \\
\hline Density of Contaminated Zone & $\mathrm{g} / \mathrm{cm}^{3}$ & 1.5 & 1.5 & .5 & RESRAD Default unless site data significantly different \\
\hline Contamination Zone Erosion Rate & $\mathrm{m} / \mathrm{yr}$ & $1.000 \mathrm{E}-03$ & $1.000 \mathrm{E}-03$ & $1.000 \mathrm{E}-03$ & RESRAD Default unless site data significantly different \\
\hline Contaminated Zone Total Porosity & - & $4.000 \mathrm{E}-01$ & $4.000 \mathrm{E}-01$ & $4.000 \mathrm{E}-01$ & RESRAD Default unless site data significantly different \\
\hline Contaminated Zone Field Capacity & - & 2.000E-01 & $2.000 \mathrm{E}-01$ & 2.000E-01 & RESRAD Default unless site data significantly different \\
\hline Contaminated Zone Hydraulic Conductivity & $\mathrm{m} / \mathrm{yr}$ & $1.000 \mathrm{E}+01$ & $1.000 \mathrm{E}+01$ & $1.000 \mathrm{E}+01$ & RESRAD Default unless site data significantly different \\
\hline Contaminated Zone b Parameter & - & $5.300 \mathrm{E}+00$ & $5.300 \mathrm{E}+00$ & $5.300 \mathrm{E}+00$ & RESRAD Default unless site data significantly different \\
\hline Average Annual Wind Speed & $\mathrm{m} / \mathrm{sec}$ & $\begin{array}{l}\text { Site } \\
\text { Specific }\end{array}$ & $\begin{array}{l}\text { Site } \\
\text { Specific }\end{array}$ & $2.000 \mathrm{E}+00$ & $\begin{array}{l}\text { Data from Air Resources Laboratory (ARL, 2005) } \\
\text { http://www.sord.nv.doe.gov/arlsord-1.htm }\end{array}$ \\
\hline Humidity in Air & $\mathrm{g} / \mathrm{m}^{3}$ & not used & not used & $8.000 \mathrm{E}+00$ & Not used with the above pathway selection \\
\hline Evapotranspiration Coefficient & - & $5.000 \mathrm{E}-01$ & $5.000 \mathrm{E}-01$ & $5.000 \mathrm{E}-01$ & $\begin{array}{l}\text { RESRAD Default not significant due to lack of } \\
\text { groundwater pathway }\end{array}$ \\
\hline Precipitation & $\mathrm{m} / \mathrm{yr}$ & $\begin{array}{l}\text { Site } \\
\text { Specific }\end{array}$ & $\begin{array}{l}\text { Site } \\
\text { Specific }\end{array}$ & $1.000 \mathrm{E}+00$ & $\begin{array}{l}\text { Data from Air Resources Laboratory (ARL, 2005) } \\
\text { http://www.sord.nv.doe.gov/arlsord-1.htm }\end{array}$ \\
\hline Irrigation & $\mathrm{m} / \mathrm{yr}$ & 0 & 0 & $2.000 \mathrm{E}-01$ & Assumes no artificial supply of water to soil \\
\hline Irrigation Mode & - & overhead & overhead & overhead & RESRAD Default \\
\hline Runoff Coefficient & - & 4.000E-01 & 4.000E-01 & 2.000E-01 & $\begin{array}{l}\text { Open Sandy Loam 30\% impervious Table } 10.1 \\
\text { (Yu, et. al., 1993) }\end{array}$ \\
\hline $\begin{array}{l}\text { Watershed Area for Nearby Stream or } \\
\text { Pond }\end{array}$ & $\mathrm{m}^{2}$ & not used & not used & $1.000 \mathrm{E}+06$ & Not used with the above pathway selection \\
\hline Accuracy for Water/Soil Computations & - & not used & not used & $1.000 \mathrm{E}-03$ & Not used with the above pathway selection \\
\hline
\end{tabular}


Table 6-2

Approved RESRAD Parameters

(Page 3 of 6 )

\begin{tabular}{|c|c|c|c|c|c|}
\hline Parameter & Units & Scenario A & Scenario B & Defaults & Reference/Rationale \\
\hline \multicolumn{6}{|l|}{ R014 Saturated Zone Hydrological Data } \\
\hline Density of Saturated Zone & $\mathrm{g} / \mathrm{cm}^{3}$ & not used & not used & $1.500 \mathrm{E}+00$ & Not used with the above pathway selection \\
\hline Saturated Zone Total Porosity & - & not used & not used & 4.000E-01 & Not used with the above pathway selection \\
\hline Saturated Zone Effective Porosity & - & not used & not used & $2.000 \mathrm{E}-01$ & Not used with the above pathway selection \\
\hline Saturated Zone Field Capacity & - & not used & not used & 2.000E-01 & Not used with the above pathway selection \\
\hline Saturated Zone Hydraulic Conductivity & $\mathrm{m} / \mathrm{yr}$ & not used & not used & $1.000 \mathrm{E}+02$ & Not used with the above pathway selection \\
\hline Saturated Zone Hydraulic Gradient & - & not used & not used & $2.000 \mathrm{E}-02$ & Not used with the above pathway selection \\
\hline Saturated Zone b Parameter & - & not used & not used & $5.300 \mathrm{E}+00$ & Not used with the above pathway selection \\
\hline Water Table Drop Rate & $\mathrm{m} / \mathrm{yr}$ & not used & not used & $1.000 \mathrm{E}-03$ & Not used with the above pathway selection \\
\hline Well Pump Intake Depth & $\mathrm{m}$ & not used & not used & $1.000 \mathrm{E}+01$ & Not used with the above pathway selection \\
\hline Model: Nondispersion or Mass-Balance & - & ND & ND & ND & RESRAD Default \\
\hline Well Pumping Rate & $\mathrm{m}^{3} / \mathrm{yr}$ & not used & not used & $2.500 \mathrm{E}+02$ & Not used with the above pathway selection \\
\hline \multicolumn{6}{|c|}{ R015 Uncontaminated and Unsaturated Strata Hydrological Data } \\
\hline Number of Unsaturated Zone Strata & - & not used & not used & 1 & Not used with the above pathway selection \\
\hline Thickness & $\mathrm{m}$ & not used & not used & $4.000 \mathrm{E}+00$ & Not used with the above pathway selection \\
\hline Soil Density & $\mathrm{g} / \mathrm{cm}^{3}$ & not used & not used & $1.500 \mathrm{E}+00$ & Not used with the above pathway selection \\
\hline Total Porosity & - & not used & not used & 4.000E-01 & Not used with the above pathway selection \\
\hline Effective Porosity & - & not used & not used & 2.000E-01 & Not used with the above pathway selection \\
\hline Field Capacity & - & not used & not used & $2.000 \mathrm{E}-01$ & Not used with the above pathway selection \\
\hline Soil-specific b Parameter & - & not used & not used & $5.300 E+00$ & Not used with the above pathway selection \\
\hline Hydraulic Conductivity & $\mathrm{m} / \mathrm{yr}$ & not used & not used & $1.000 \mathrm{E}+01$ & Not used with the above pathway selection \\
\hline
\end{tabular}


Table 6-2

Approved RESRAD Parameters

(Page 4 of 6)

\begin{tabular}{|c|c|c|c|c|c|}
\hline Parameter & Units & Scenario A & Scenario B & Defaults & Reference/Rationale \\
\hline \multicolumn{6}{|c|}{ R016 Distribution Coefficients and Leach Rates } \\
\hline Contaminated Zone $\mathrm{K}_{\mathrm{d}}$ (all Zones) & $\mathrm{cm}^{3} / \mathrm{g}$ & & & & RESRAD Defaults \\
\hline Saturated Leach Rate & /yr & 0.0 & 0.0 & 0.0 & Not used \\
\hline Solubility Constant & - & 0.0 & 0.0 & 0.0 & Not used \\
\hline \multicolumn{6}{|l|}{ R017 Inhalation and External Gamma } \\
\hline Inhalation Rate & $\mathrm{m}^{3} / \mathrm{yr}$ & $8.400 E+03$ & $1.230 \mathrm{E}+04$ & $8.400 E+03$ & $\begin{array}{l}\text { RESRAD Default and for an individual performing outdoor } \\
\text { activities, a typical activity mix can consist of } 37 \% \text { at a } \\
\text { moderate activity level, } 28 \% \text { at both resting and light } \\
\text { activity levels, and } 7 \% \text { at a heavy activity level, which } \\
\text { results in a } 1.4 \mathrm{~m}^{3} / \mathrm{h}\left(12,300 \mathrm{~m}^{3} / \mathrm{yr}\right) \text { inhalation rate. } \\
(\mathrm{Yu} \text {, et. al., } 1993)\end{array}$ \\
\hline Mass Loading for Inhalation & $\mathrm{g} / \mathrm{m}^{3}$ & $6.00 \mathrm{E}-04$ & $6.00 \mathrm{E}-04$ & $1 \mathrm{E}-04$ & $\begin{array}{l}\text { The estimated mass loading for construction activities. } \\
\text { (Yu, et. al., 1993) }\end{array}$ \\
\hline Exposure Duration & $\mathrm{yr}$ & 25 & 25 & 30 & Standard for Industrial/Commercial Scenario \\
\hline Shielding Factor Inhalation & - & 1 & 1 & 0.4 & Assumes no indoor time fraction. \\
\hline Shielding Factor External Gamma & - & 1 & 1 & 0.7 & Assumes no indoor time fraction. \\
\hline Fraction of Time Spent Indoors & - & 0.0 & 0.0 & 0.5 & Assumes no indoor time fraction. \\
\hline Fraction of Time Spent Outdoors & - & 0.228 & 0.038 & 0.25 & $\begin{array}{l}\text { Based on Industrial/Commercial use scenarios for } \\
\text { standard occupancy and low occupancy. }\end{array}$ \\
\hline Shape Factor & - & 1.0 & 1.0 & 1.0 & RESRAD Default \\
\hline
\end{tabular}


Table 6-2

Approved RESRAD Parameters

(Page 5 of 6 )

\begin{tabular}{|c|c|c|c|c|c|}
\hline Parameter & Units & Scenario A & Scenario B & Defaults & Reference/Rationale \\
\hline \multicolumn{6}{|c|}{ R018 Ingestion Pathway Data, Dietary Parameters } \\
\hline $\begin{array}{l}\text { Fruits, Vegetables, and Grain } \\
\text { Consumption }\end{array}$ & $\mathrm{kg} / \mathrm{yr}$ & not used & not used & $1.600 \mathrm{E}+02$ & Not used with the above pathway selection \\
\hline Leafy Vegetable Consumption & $\mathrm{kg} / \mathrm{yr}$ & not used & not used & $1.400 \mathrm{E}+01$ & Not used with the above pathway selection \\
\hline Milk Consumption & L/yr & not used & not used & $9.200 \mathrm{E}+01$ & Not used with the above pathway selection \\
\hline Meat and Poultry Consumption & $\mathrm{kg} / \mathrm{yr}$ & not used & not used & $6.300 \mathrm{E}+01$ & Not used with the above pathway selection \\
\hline Fish Consumption & $\mathrm{kg} / \mathrm{yr}$ & not used & not used & $5.400 \mathrm{E}+00$ & Not used with the above pathway selection \\
\hline Other Seafood Consumption & $\mathrm{kg} / \mathrm{yr}$ & not used & not used & $9.000 \mathrm{E}-01$ & Not used with the above pathway selection \\
\hline Soil Ingestion Rate & $\mathrm{g} / \mathrm{yr}$ & $1.752 E+02$ & $1.752 E+02$ & 36.5 & 480 mg/day (EPA, 1991) \\
\hline Drinking Water Intake & L/yr & not used & not used & $5.100 \mathrm{E}+02$ & Not used with the above pathway selection \\
\hline Drinking Water Contaminated Fraction & - & not used & not used & $1.000 \mathrm{E}+00$ & Not used with the above pathway selection \\
\hline Household Water Contaminated Fraction & - & not used & not used & $1.000 \mathrm{E}+00$ & Not used with the above pathway selection \\
\hline Livestock Water Contaminated Fraction & - & not used & not used & $1.000 \mathrm{E}+00$ & Not used with the above pathway selection \\
\hline Irrigation Water Contaminated Fraction & - & not used & not used & $1.000 \mathrm{E}+00$ & Not used with the above pathway selection \\
\hline Aquatic Food Contamination Fraction & - & not used & not used & $5.000 \mathrm{E}-01$ & Not used with the above pathway selection \\
\hline Plant Food Contamination Fraction & - & not used & not used & -1 & Not used with the above pathway selection \\
\hline Meat Contamination Fraction & - & not used & not used & -1 & Not used with the above pathway selection \\
\hline Milk Contamination Fraction & - & not used & not used & -1 & Not used with the above pathway selection \\
\hline \multicolumn{6}{|l|}{ R019 Ingestion Pathway Data, Nondietary } \\
\hline Livestock Fodder Intake for Meat & $\mathrm{kg} /$ day & not used & not used & $6.800 \mathrm{E}+01$ & Not used with the above pathway selection \\
\hline Livestock Fodder Intake for Milk & $\mathrm{kg} /$ day & not used & not used & $5.500 \mathrm{E}+01$ & Not used with the above pathway selection \\
\hline Livestock Water Intake for Meat & L/day & not used & not used & $5.000 \mathrm{E}+01$ & Not used with the above pathway selection \\
\hline Livestock Water Intake for Milk & L/day & not used & not used & $1.600 \mathrm{E}+02$ & Not used with the above pathway selection \\
\hline
\end{tabular}


Table 6-2

Approved RESRAD Parameters

(Page 6 of 6 )

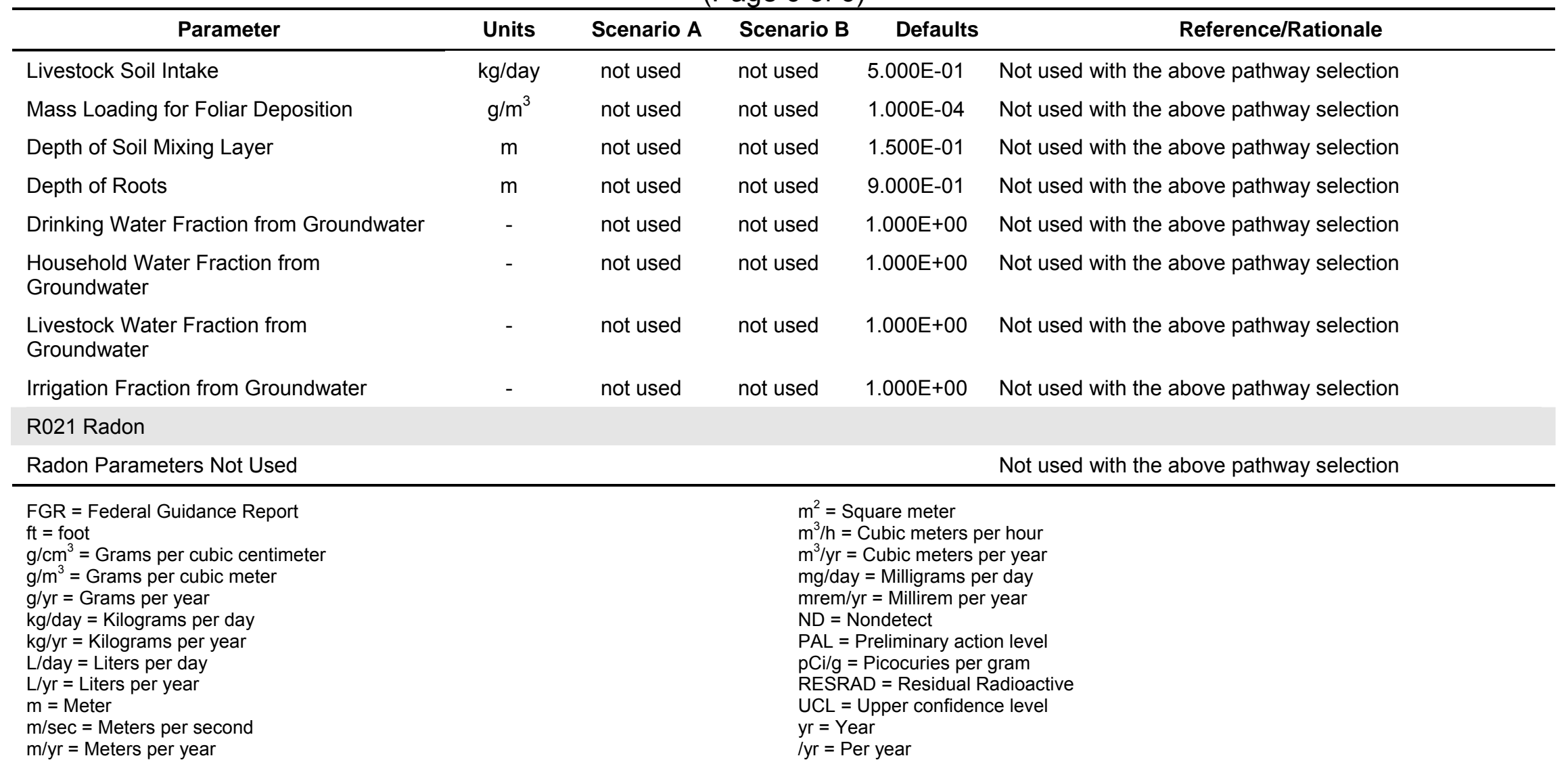

\section{Uncontrolled When Printed}




\subsection{Residual Radioactive Material Guideline}

The residual radioactive material guideline represents the concentration of residual radioactive material that can remain in place and still allow use of that area without radiological restrictions. Using site-specific parameters and sample analysis results, the radioactive material guideline, $\mathrm{G}$, can be calculated for a given dose limit of $\mathrm{H}_{\mathrm{EL}}$ for an individual as follows;

$$
\mathrm{G}=\mathrm{H}_{\mathrm{EL}} / \mathrm{DSR}
$$

where DSR is the total dose/source concentration ratio. The dose limit $\mathrm{H}_{\mathrm{EL}}$, used to derive the residual radioactive material guideline is $25 \mathrm{mrem} / \mathrm{yr}$.

Single radionuclide guidelines are calculated for individual radionuclides such that the annual dose to industrial/construction workers at the site should not exceed an annual dose limitation of $25 \mathrm{mrem} / \mathrm{yr}$. Sites contaminated with two or more radionuclides (i.e., a mixture of radionuclides) require further evaluation to ensure that collective exposures from individual radionuclides do not exceed the $25 \mathrm{mrem} / \mathrm{yr}$ annual dose constraint. This evaluation is performed using a sum of the fractions method. The initial soil concentration of each radionuclide is divided by the single radionuclide guideline for that radionuclide to produce a ratio. These ratios are then summed. If the sum is less than or equal to unity, then the collective annual dose from all radionuclides at the site should not exceed the $25 \mathrm{mrem} / \mathrm{yr}$ annual dose constraint. If the sum does exceed unity, the annual dose to industrial/construction workers could exceed the $25 \mathrm{mrem} / \mathrm{yr}$ dose constraint, even if the concentrations of residual radionuclides at the site are below the single radionuclide guideline values. For sites where the sum of the ratios exceeds unity, residual radioactive material guidelines for mixtures of radionuclides are calculated such that the following equation is satisfied;

$$
\overline{\mathrm{M}}=\sum_{\mathrm{i}} \overline{\mathrm{S}}_{\mathrm{i}}\left(\mathrm{t}_{\mathrm{o}}\right) / \mathrm{G}_{\mathrm{i}}\left(\mathrm{t}_{\mathrm{m}}\right) \leq 1
$$

Where: $\quad \begin{array}{lll}\overline{\mathrm{M}} & = & \text { average mixture sum (dimensionless) } \\ \overline{\mathrm{S}}_{\mathrm{i}}\left(\mathrm{t}_{\mathrm{o}}\right) & = & \begin{array}{l}\text { initial concentration of the } i \text { th principal radionuclide } \\ \text { averaged over an area determined by scenario activities }\end{array} \\ \mathrm{G}_{\mathrm{i}}\left(\mathrm{t}_{\mathrm{m}}\right) & = & \begin{array}{l}\text { single radionuclide soil concentration guideline for the } i t h \\ \text { principal radionuclide at time } \mathrm{t} \text { maximum. }\end{array}\end{array}$

For a site where the sum of the ratios does not exceed unity, the residual radioactive guidelines for single radionuclides are the radionuclide concentrations to be used as the FAL. For sites where the sum of the ratios exceeds unity, the residual radioactive guidelines for mixtures of radionuclides are mathematically adjusted so that the above equation is satisfied. Those adjusted values are then used as the FAL. 


\subsection{RESRAD Calculations for CAU 476, Area 12 T-Tunnel Muckpile}

This section discuses the RESRAD calculations and results for CAU 476.

\subsection{Selection of RESRAD Exposure Scenario}

Scenario B was selected as the exposure scenario for the CAU 476 because of the remote location of the site. Because Scenario B parameters will be used for these calculations, a Use Restriction will be implemented at closure that will state the use scenario and the requirement for an occupant agency or entity to re-evaluate the closure if site use changes to fit the parameters of Scenario A.

\subsection{User Input Parameters}

The RESRAD default parameters that were modified for the calculations performed for CAU 476 in this report and the site-specific values entered are presented in Table 7-1. A complete list of the RESRAD default parameters and the site-specific parameters used for CAU 476 is provided in Table A.1 of Attachment A. The initial radionuclide concentrations used for analyses are those listed in Table 4-1.

\subsection{Radionuclide Concentrations and Dose Estimates}

The maximum dose results from RESRAD calculations for the CAU 476 is $35.56 \mathrm{mrem} / \mathrm{yr}$ occurring at year zero (current year). The detailed RESRAD results for all three portions of this CAS are provided in Exhibit 1, RESRAD Summary Report: CAU 476.

Uncertainty in the derivation of dose estimates and dose/source contribution ratios comes from the distribution of possible input parameter values, as well as uncertainty in the conceptual model used to represent the site. The pathway contributing to the total annual dose at the time of maximum dose occurs are almost all (99.69 percent) for external exposure, 0.18 percent for inhalation, and 0.13 percent for soil ingestion pathways. Therefore, uncertainties in the following parameters: Erosion rates, thickness of contaminated zone, occupancy factors, and wind speed have the greatest significance on the model predictions.

The maximum dose contributions and total dose/source concentration ratios for the muckpile under Scenario B parameters have been predicted to occur at year zero. The calculated maximum dose contributions for all considered pathways are presented in Table 7-2. Figure 7-1 shows that the TEDE to remote worker for the considered pathway decreases within the $25 \mathrm{mrem} / \mathrm{yr}$ dose constraint only at year 13. The dose from Cs-137, the dominating contributing radionuclide, at year 13 is $24.59 \mathrm{mrem} / \mathrm{yr}$ and doses from all radionuclides drop to $24.80 \mathrm{mrem} / \mathrm{yr}$ for year 13 .

Because the radionuclide concentrations found at this site pose a dose level above the 25-mrem/yr constraint under the current site conditions, remediation alternative should be considered for the site. Nonetheless, controls that minimize the spread of radioactive contamination into uncontaminated areas and reduced erosion rate are recommended for this site. 
Unless the muckpile is removed during remediation, the site should be radiologically posted in accordance with applicable regulation and procedures.

\subsection{Residual Radioactive Material Guidelines for CAU 476}

The sum of the ratios for CAU 476 exceeds unity. Table 7-3 presents the calculations results for deriving guidelines for radionuclides for this CAU. The FALs for the CAU 476 scenario are the RESRAD material guideline values for mixture radionuclides. 
Table 7-1

RESRAD Parameters Input Values for CAU 476

(Page 1 of 2)

\begin{tabular}{|c|c|c|c|c|}
\hline Parameter & Units & CAU 476 & Defaults & Reference/Rationale \\
\hline Area of $\mathrm{CZ}$ & $\mathrm{m}^{2}$ & $1.000 \mathrm{E}+02$ & $1.000 \mathrm{E}+04$ & Estimated using the site boundary \\
\hline Thickness of $\mathrm{CZ}$ & $\mathrm{m}$ & $1.500 \mathrm{E}-01$ & $2.000 \mathrm{E}+00$ & Top layer of the contamination soil \\
\hline Principal radionuclides & $\mathrm{pCi} / \mathrm{g}$ & See Table 7-2 & 0.0 & $\begin{array}{l}\text { Initial concentrations are the maximum concentrations from } \\
\text { sample results: maximum for biased sample or average for } \\
\text { random sample. }\end{array}$ \\
\hline Average Annual Wind Speed & $\mathrm{m} / \mathrm{sec}$ & 3.4 & $2.000 \mathrm{E}+00$ & Data from Air Resource Laboratory (2005) \\
\hline Precipitation & $\mathrm{m} / \mathrm{yr}$ & $3.260 \mathrm{E}-01$ & $1.000 \mathrm{E}+00$ & Data from Air Resources Laboratory \\
\hline Runoff Coefficient & - & $4.000 \mathrm{E}-01$ & $2.000 \mathrm{E}-01$ & $\begin{array}{l}\text { Open Sandy Loam 30\% impervious Table } 10.1 \\
\text { (Yu, et al., 1993) }\end{array}$ \\
\hline Inhalation Rate & $\mathrm{m}^{3} / \mathrm{yr}$ & $1.230 \mathrm{E}+04$ & $8.400 \mathrm{E}+03$ & $\begin{array}{l}\text { RESRAD Default and for an individual performing outdoor } \\
\text { activities, a typical activity mix can consist of } 37 \% \text { at a } \\
\text { moderate activity level, } 28 \% \text { at both resting and light activity } \\
\text { levels, and } 7 \% \text { at a heavy activity level, which results in a } \\
\left.1.4 \mathrm{~m}^{3} / \mathrm{h}\left(12,300 \mathrm{~m}^{3} / \mathrm{yr}\right) \text { inhalation rate. (Yu, et al., } 1993\right)\end{array}$ \\
\hline Mass Loading for Inhalation & $\mathrm{g} / \mathrm{m}^{3}$ & $6.00 \mathrm{E}-04$ & $1 \mathrm{E}-04$ & $\begin{array}{l}\text { The estimated mass loading for construction activities. } \\
\text { (Yu, et al., 1993) }\end{array}$ \\
\hline Exposure Duration & $\mathrm{yr}$ & 25 & 30 & Standard for Industrial/Commercial Scenario \\
\hline Shielding Factor Inhalation & - & 1.0 & 0.4 & Assumes no indoor time fraction \\
\hline Shielding Factor External Gamma & - & 1.0 & 0.7 & Assumes no indoor time fraction \\
\hline Fraction of Time Spent Indoors & - & 0.0 & 0.5 & Assumes no indoor time fraction \\
\hline Fraction of Time Spent Outdoors & - & 0.038 & 0.25 & $\begin{array}{l}\text { Scenario specific based on Industrial/Commercial Use } \\
\text { Scenarios for standard occupancy and low occupancy. }\end{array}$ \\
\hline Soil Ingestion Rate & $\mathrm{g} / \mathrm{yr}$ & $1.752 E+02$ & 36.5 & EPA, 1991; 480 mg/day \\
\hline
\end{tabular}


Table 7-1

RESRAD Parameters Input Values for CAU 476

(Page 2 of 2)

\begin{tabular}{|c|c|c|c|}
\hline Parameter & CAU 476 & Defaults & Reference/Rationale \\
\hline $\begin{array}{l}\mathrm{CZ}=\text { Contaminated Zone } \\
\mathrm{g} / \mathrm{m}^{3}=\text { Grams per cubic meter } \\
\mathrm{g} / \mathrm{yr}=\text { Grams per year } \\
\mathrm{m}=\text { Meter } \\
\mathrm{m}^{2}=\text { Square meter } \\
\mathrm{m} / \mathrm{sec}=\text { Meters per second } \\
\mathrm{m} / \mathrm{yr}=\text { Meters per year }\end{array}$ & & $\begin{array}{l}\mathrm{m}^{3} / \mathrm{h}=\text { Cubic meters per hour } \\
\mathrm{m}^{3} / \mathrm{yr}=\text { Cubic meters per year } \\
\mathrm{mg} / \text { day = Milligrams per day } \\
\mathrm{pCi} / \mathrm{g}=\text { Picocuries per gram } \\
\mathrm{RESRAD}=\text { Residual Radioactive } \\
\mathrm{yr}=\text { Year }\end{array}$ & \\
\hline
\end{tabular}


Table 7-2

Maximum Dose Contributions for CAU 476

Using Scenario B (dose as mrem/yr)

\begin{tabular}{|c|c|c|c|c|c|c|c|c||}
\hline \multirow{2}{*}{ Radionuclide } & \multicolumn{2}{|c|}{ Ground } & \multicolumn{2}{c|}{ Inhalation } & \multicolumn{2}{c|}{ Soil } & \multicolumn{2}{c||}{ Total } \\
\cline { 2 - 9 } & $\begin{array}{c}\text { Annual } \\
\text { Dose }\end{array}$ & Fraction & $\begin{array}{c}\text { Annual } \\
\text { Dose }\end{array}$ & Fraction & $\begin{array}{c}\text { Annual } \\
\text { Dose }\end{array}$ & Fraction & $\begin{array}{c}\text { Annual } \\
\text { Dose }\end{array}$ & Fraction \\
\hline \hline Americium-241 & $3.828 \mathrm{E}-03$ & 0.0001 & $1.394 \mathrm{E}-02$ & 0.0004 & $6.690 \mathrm{E}-03$ & 0.0002 & $2.446 \mathrm{E}-02$ & 0.0007 \\
\hline Cobalt-60 & $6.732 \mathrm{E}-01$ & 0.0189 & $4.094 \mathrm{E}-06$ & 0.0000 & $2.943 \mathrm{E}-05$ & 0.0000 & $6.732 \mathrm{E}-01$ & 0.0189 \\
\hline Cesium-137 & $3.469 \mathrm{E}+01$ & 0.9755 & $1.366 \mathrm{E}-04$ & 0.0000 & $1.253 \mathrm{E}-02$ & 0.0004 & $3.470 \mathrm{E}+01$ & 0.9758 \\
\hline Plutonium-238 & $5.335 \mathrm{E}+06$ & 0.0000 & $4.559 \mathrm{E}-03$ & 0.0001 & $2.178 \mathrm{E}-03$ & 0.0001 & $6.742 \mathrm{E}-03$ & 0.0002 \\
\hline Plutonium-239 & $8.623 \mathrm{E}-05$ & 0.0000 & $4.668 \mathrm{E}-02$ & 0.0013 & $2.255 \mathrm{E}-02$ & 0.0006 & $6.932 \mathrm{E}-02$ & 0.0019 \\
\hline Antimony-125 & $7.627 \mathrm{E}-02$ & 0.0021 & $1.788 \mathrm{E}-07$ & 0.0000 & $2.753 \mathrm{E}-06$ & 0.0000 & $7.628 \mathrm{E}-02$ & 0.0021 \\
\hline Strontium-90 & $8.917 \mathrm{E}-03$ & 0.0003 & $1.893 \mathrm{E}-04$ & 0.0000 & $1.294 \mathrm{E}-03$ & 0.0000 & $1.040 \mathrm{E}-02$ & 0.0003 \\
\hline Total & $3.545 \mathrm{E}+01$ & 0.9969 & $6.551 \mathrm{E}-02$ & 0.0018 & $4.527 \mathrm{E}-02$ & 0.0013 & $3.556 \mathrm{E}+01$ & 1.0000 \\
\hline
\end{tabular}

$\mathrm{mrem} / \mathrm{yr}=$ Millirem per year 


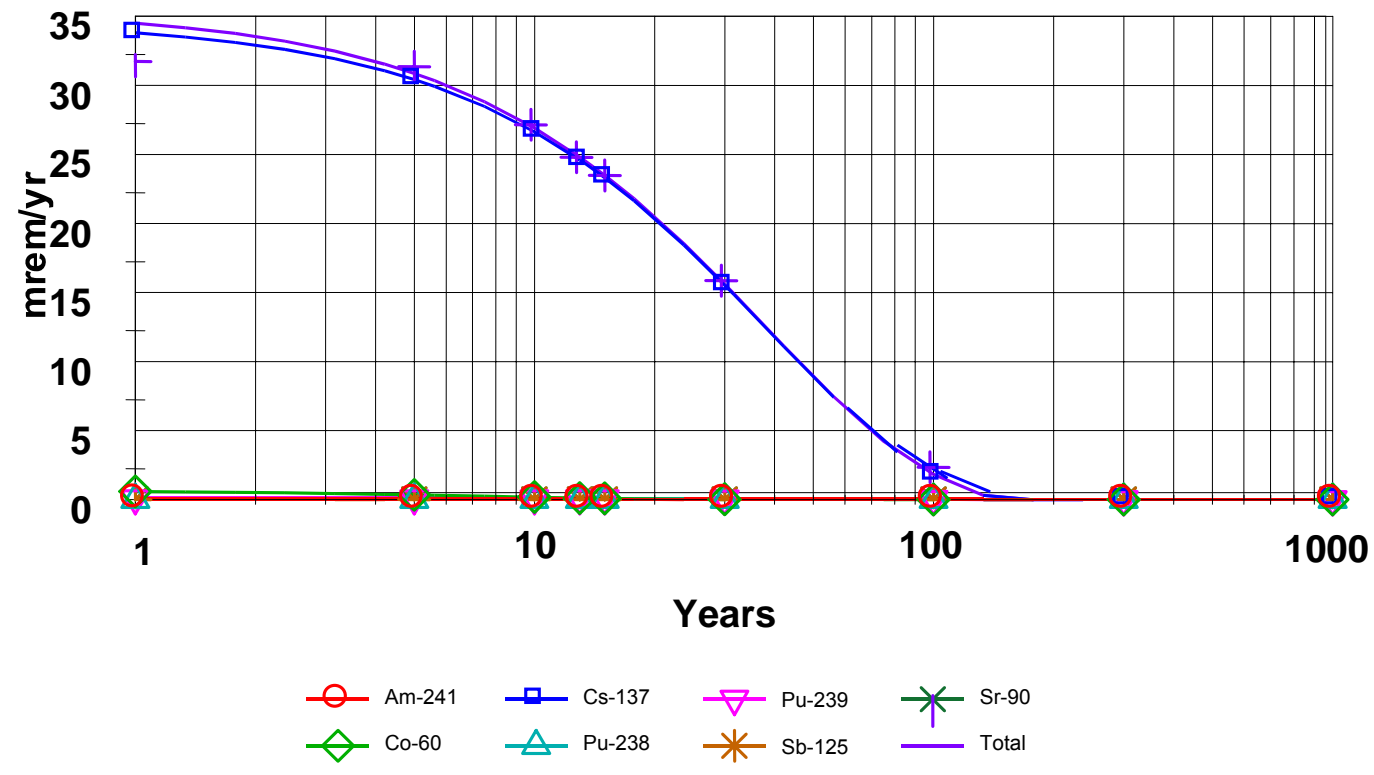

DOSE: All Radionuclides Summed, All Pathways Summed
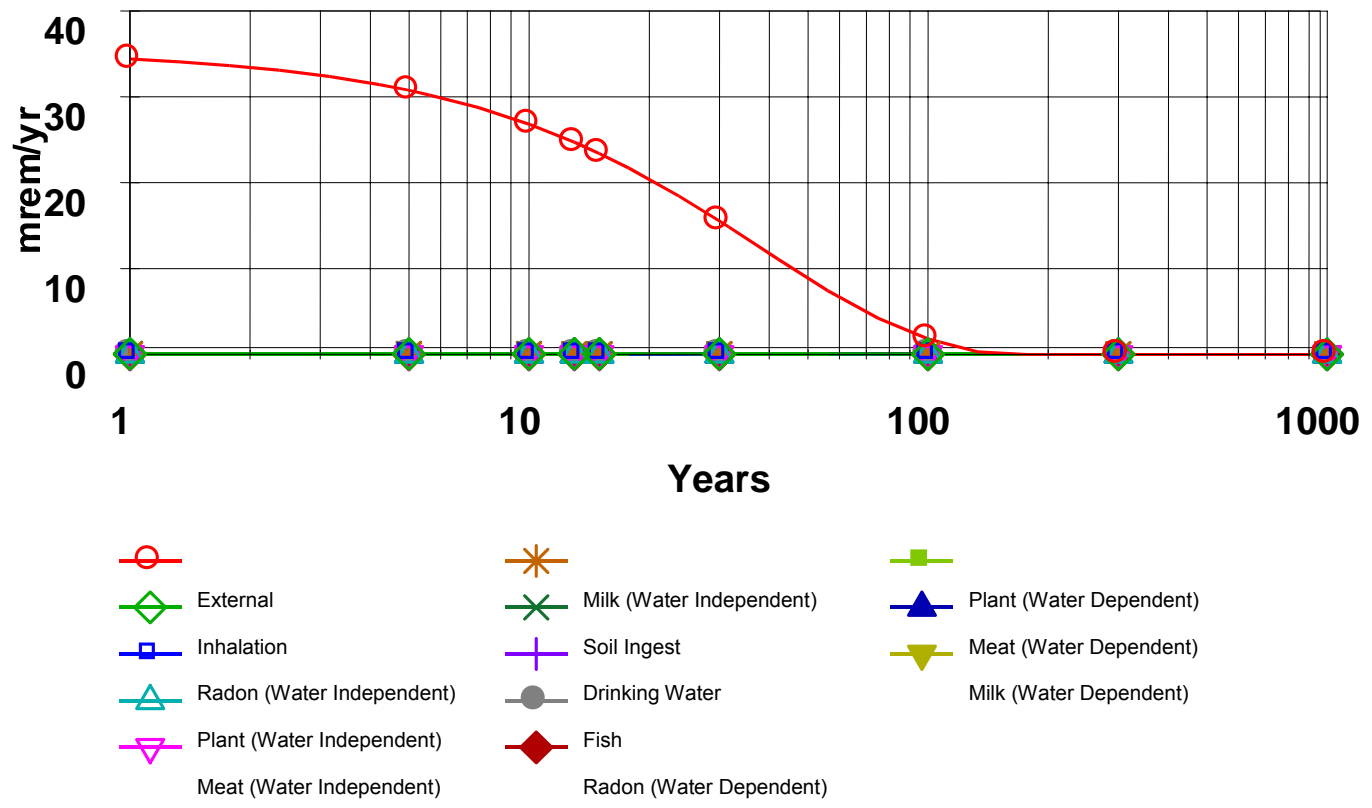

DOSE: All Radionuclides Summed, Component Pathways

Figure 7-1

CAU 476 Dose Rate Per Year All Radionuclides Summed, All Pathways Summed and Component Pathways 
Table 7-3

CAU 476 Sum of Fractions and Proportional Scaling and Final Action Level Determination

\begin{tabular}{|c|c|c|c|c|c|c|}
\hline Radionuclide & $\begin{array}{c}\text { Initial } \\
\text { Radionuclide } \\
\text { Concentration } \\
\text { (pCi/g) }\end{array}$ & $\begin{array}{c}\text { Contribution } \\
\%\end{array}$ & $\begin{array}{c}\text { Single } \\
\text { Radionuclide } \\
\text { Guidelines } \\
\text { (pCi/g) }\end{array}$ & $\begin{array}{l}\text { Ratio for } \\
\text { Single } \\
\text { Radionuclide } \\
\text { Guideline }\end{array}$ & $\begin{array}{c}\text { Mixture } \\
\text { Radionuclides } \\
\text { Guidelines }^{\mathrm{a}}\end{array}$ & $\begin{array}{l}\text { Ratio for Mixture } \\
\text { Radionuclide } \\
\text { Guidelines } \\
\text { (FAL) }\end{array}$ \\
\hline Americium-241 & $2.8000 \mathrm{E}+00$ & 6.7765E-03 & $2.8620 \mathrm{E}+03$ & 9.7834E-04 & $2.7387 \mathrm{E}+00$ & 9.5690E-04 \\
\hline Cobalt-60 & $1.7600 E+00$ & 4.2595E-03 & $6.5360 E+01$ & 2.6928E-02 & $1.7214 \mathrm{E}+00$ & 2.6338E-02 \\
\hline Cesium-137 & $3.8200 E+02$ & $9.2451 \mathrm{E}-01$ & $2.7520 E+02$ & $1.3881 \mathrm{E}+00$ & $3.7363 E+02$ & 1.3577E+00 \\
\hline Plutonium-238 & $1.0300 E+00$ & 2.4928E-03 & $3.8190 E+03$ & 2.6970E-04 & $1.0074 \mathrm{E}+00$ & 2.6380E-04 \\
\hline Plutonium-239 & $9.6000 E+00$ & 2.3234E-02 & $3.4620 E+03$ & 2.7730E-03 & $9.3897 E+00$ & 2.7122E-03 \\
\hline Antimony-125 & $3.0000 E+00$ & 7.2606E-03 & $9.8330 E+02$ & 3.0510E-03 & $2.9343 E+00$ & $2.9841 \mathrm{E}-03$ \\
\hline Strontium-90 & $1.3000 E+01$ & 3.1463E-02 & $3.1250 E+04$ & 4.1600E-04 & $1.2715 \mathrm{E}+01$ & 4.0689E-04 \\
\hline Total & 4.1319E+02 & $\mathrm{N} / \mathrm{A}$ & 4.2717E+04 & $1.4225 \mathrm{E}+00^{b}$ & $4.0414 \mathrm{E}+02$ & 1.3913E+00 \\
\hline
\end{tabular}

${ }^{a}$ Single radionuclide guidelines apply to areas uniformly contaminated with a single radionuclide. The mixture radionuclide guidelines apply to areas uniformly contaminated with a mixture of radionuclides. The FALs for CAU 476 are the radionuclide guidelines for mixture radionuclides (i.e., Mixture Radionuclide Guidelines column).

bunity $>1$

$\mathrm{FAL}=$ Final action level

$\mathrm{N} / \mathrm{A}=$ Not applicable

$\mathrm{pCi} / \mathrm{g}=$ Picocuries per gram 


\subsection{References}

ARL, see Air Resources Laboratory.

Air Resources Laboratory. 2005. "Climatological Information and Data." As accessed at http://www.sord.nv.doe.gov.

DOE, see U.S. Department of Energy.

DOE/NV, see U.S. Department of Energy, Nevada Operations Office.

DTRA, see Defense Threat Reduction Agency.

Defense Threat Reduction Agency. 2000. Corrective Action Investigation Plan for Corrective Action Unit 476: Area 12 T-Tunnel Muckpile, Nevada Test Site, Nevada, Rev. 0.

EPA, see U.S. Environmental Protection Agency.

Lawrence, A., Office of Environmental Policy \& Guidance. 2002. Memorandum to Distribution entitled, "Radiation Risk Estimation from Total Effective Dose Equivalents (TEDEs)," (EH-412-2002-1), 9 August.

NCRP, see National Council on Radiation Protection and Measurements.

NDEP, see Nevada Division of Environmental Protection.

National Council on Radiation Protection and Measurements. 1999. Recommended Screening Limits for Contaminated Surface Soil and Review of Factors Relevant to Site-Specific Studies/National Council on Radiation Protection and Measurements, NCRP Report No. 129. Bethesda, MD.

Nevada Division of Environmental Protection. 2004. Review of Industrial Sites Project Document "Guidance for Calculating Industrial Sites Project Remediation Goals for Radionuclides in Soil Using the Residual Radiation (RESRAD) Computer Code."

SNJV GIS Systems, see Stoller-Navarro Joint Venture Geographic Information Systems.

Stoller-Navarro Joint Venture Geographic Information Systems. 2007. ESRI Arc GIS Software.

U.S. Department of Energy. 1993. Radiation Protection of the Public and the Environment, DOE 5400.5, Change 2. Washington, DC.

U.S. Department of Energy. 2002. Draft Implementation Guide, Control and Release of Property with Residual Radioactive Material, for Use with DOE 5400.5, Radiation Protection of the Public and the Environment, DOE G 441.1-XX. Washington, DC. 
U.S. Department of Energy, Nevada Operations Office. 1996. Final Environmental Impact Statement for the Nevada Test Site and Off-Site Locations in the State of Nevada, DOE/EIS 0243. Las Vegas, NV.

U.S. Department of Energy, Nevada Operations Office. 1998. Nevada Test Site Resource Management Plan. DOE/NV--518, Las Vegas, NV.

U.S. Environmental Protection Agency. 1991. Human Health Evaluation Manual Supplemental Guidance: “Standard Default Exposure Factors.” OSWER Directive 9285.6-03.

U.S. Environmental Protection Agency. 1992. Guidance on Risk Managers and Risk Assessors. Memorandum from F.H. Habicht, Deputy Administrator, Washington, DC

U.S. Environmental Protection Agency. 1994. Data Quality Objectives Process. EPA QA/G-4. Washington, DC

Yu C., C. Loureiro, C.C. Cheng, L.G. Jones, Y.Y. Wang, Y.P. Chia, and E. Faillace. 1993. Data Collection Handbook To Support Modeling the Impacts of Radioactive Material in Soil, ANL/EAIS-8. Chicago, IL: Environmental Assessment Division, Argonne National Laboratory.

Yu C., A.J. Zielen, J.J Cheng, D.J. LePoire., E. Gnanapragasam, S. Kamboj, J. Arnish, A. Wallo III, W.A. Williams, and H. Peterson. 2001. User's Manual for RESRAD Version 6, ANL/EAD-4, Argonne National Laboratory, Environmental Assessment Division, Argonne, IL. 


\section{Attachment A}

\section{RESRAD Parameters Used for Analysis of CAU 476 Site}

The parametric values used in the RESRAD code for the analysis of the CAU 476 site are listed in Table A-1. Some parameters are site specific, while other values are default RESRAD values. The dose conversion factors used for inhalation and ingestion were the default Federal Guidance Report 13 morbidity values and correspond to the guidance and recommendations per the August 9, 2002, memorandum from A. Lawrence, Office of Environmental Policy \& Guidance, to Distribution, titled "Radiation Risk Estimation from Total Effective Dose Equivalents (TEDEs)” (EH-412-2002-1) (Lawrence, 2002). 
Table A-1

RESRAD Parameters

(Page 1 of 6)

\begin{tabular}{|c|c|c|c|c|}
\hline Parameter & Units & CAU 476 & Defaults & Reference/Rationale \\
\hline \multicolumn{5}{|l|}{ R011 Contaminated Zone (CZ) } \\
\hline Area of $\mathrm{CZ}$ & $\mathrm{m}^{2}$ & $1.00 \mathrm{E}+02$ & $1.000 \mathrm{E}+04$ & $10 \times 10 \mathrm{~m}$ specific location area \\
\hline Thickness of CZ & $\mathrm{m}$ & $1.200 \mathrm{E}+00$ & $2.000 \mathrm{E}+00$ & $\begin{array}{l}\text { Maximum depth from contaminated } \\
\text { samples }\end{array}$ \\
\hline Length Parallel to Aquifer Flow & $\mathrm{m}$ & not used & $1.000 \mathrm{E}+02$ & Not Used \\
\hline Radiation Dose Limit & $\mathrm{mrem} / \mathrm{yr}$ & $2.5 \mathrm{E}+001$ & $2.5 \mathrm{E}+001$ & RESRAD Default (Yu, et al., 1993) \\
\hline Elapsed Time Since Placement of Material & $\mathrm{yr}$ & 0.0 & 0.0 & RESRAD Default \\
\hline \multicolumn{5}{|l|}{ R012 Initial Principal Radionuclide } \\
\hline Principal radionuclides & $\mathrm{pCi} / \mathrm{g}$ & See Table 7.2 & 0.0 & Site specific \\
\hline
\end{tabular}


Table A-1

RESRAD Parameters

(Page 2 of 6)

\begin{tabular}{|c|c|c|c|c|}
\hline Parameter & Units & CAU 476 & Defaults & Reference/Rationale \\
\hline \multicolumn{5}{|c|}{ R013 Cover and Contaminated Zone Hydrological Data } \\
\hline Cover Depth & $\mathrm{m}$ & 0.0 & 0.0 & No Cover Assumed \\
\hline Density of Cover Material & $\mathrm{g} / \mathrm{cm}^{3}$ & not used & 1.5 & No Cover Assumed \\
\hline Cover Depth Erosion Rate & $\mathrm{m} / \mathrm{yr}$ & not used & 1.000E-03 & No Cover Assumed \\
\hline Density of Contaminated Zone & $\mathrm{g} / \mathrm{cm}^{3}$ & 1.5 & 1.5 & RESRAD Default \\
\hline Contamination Zone Erosion Rate & $\mathrm{m} / \mathrm{yr}$ & 1.000E-03 & 1.000E-03 & RESRAD Default \\
\hline Contaminated Zone Total Porosity & - & 4.000E-01 & $4.000 E-01$ & RESRAD Default \\
\hline Contaminated Zone Field Capacity & - & 2.000E-01 & $2.000 \mathrm{E}-01$ & RESRAD Default \\
\hline Contaminated Zone Hydraulic Conductivity & $\mathrm{m} / \mathrm{yr}$ & $1.000 \mathrm{E}+01$ & $1.000 \mathrm{E}+01$ & RESRAD Default \\
\hline Contaminated Zone b Parameter & - & $5.300 \mathrm{E}+00$ & $5.300 \mathrm{E}+00$ & RESRAD Default \\
\hline Average Annual Wind Speed & $\mathrm{m} / \mathrm{sec}$ & 3.4 & $2.000 \mathrm{E}+00$ & Data from Air Resource Laboratory (2005) \\
\hline Humidity in Air & $\mathrm{g} / \mathrm{m}^{3}$ & not used & $8.000 \mathrm{E}+00$ & Not used \\
\hline Evapotranspiration Coefficient & - & $5.000 \mathrm{E}-01$ & 5.000E-01 & RESRAD Default \\
\hline Precipitation & $\mathrm{m} / \mathrm{yr}$ & $3.260 \mathrm{E}-01$ & $1.000 \mathrm{E}+00$ & Data from Air Resources Laboratory \\
\hline Irrigation & $\mathrm{m} / \mathrm{yr}$ & 2.000E-01 & $2.000 \mathrm{E}-01$ & RESRAD Default \\
\hline Irrigation Mode & - & overhead & overhead & RESRAD Default \\
\hline Runoff Coefficient & - & 4.000E-01 & $2.000 \mathrm{E}-01$ & $\begin{array}{l}\text { Open Sandy Loam 30\% impervious Table } \\
10.1 \text { (Yu, et al., 1993) }\end{array}$ \\
\hline Watershed Area for Nearby Stream or Pond & $\mathrm{m}^{2}$ & not used & $1.000 E+06$ & Not used \\
\hline Accuracy for Water/Soil Computations & - & not used & 1.000E-03 & Not used \\
\hline
\end{tabular}


Table A-1

RESRAD Parameters

(Page 3 of 6)

\begin{tabular}{|c|c|c|c|c|}
\hline Parameter & Units & CAU 476 & Defaults & Reference/Rationale \\
\hline \multicolumn{5}{|l|}{ R014 Saturated Zone Hydrological Data } \\
\hline Density of Saturated Zone & $\mathrm{g} / \mathrm{cm}^{3}$ & not used & $1.500 \mathrm{E}+00$ & Not used \\
\hline Saturated Zone Total Porosity & - & not used & 4.000E-01 & Not used \\
\hline Saturated Zone Effective Porosity & - & not used & $2.000 \mathrm{E}-01$ & Not used \\
\hline Saturated Zone Field Capacity & - & not used & 2.000E-01 & Not used \\
\hline Saturated Zone Hydraulic Conductivity & $\mathrm{m} / \mathrm{yr}$ & not used & $1.000 \mathrm{E}+02$ & Not used \\
\hline Saturated Zone Hydraulic Gradient & - & not used & 2.000E-02 & Not used \\
\hline Saturated Zone b Parameter & - & not used & $5.300 \mathrm{E}+00$ & Not used \\
\hline Water Table Drop Rate & $\mathrm{m} / \mathrm{yr}$ & not used & 1.000E-03 & Not used \\
\hline Well Pump Intake Depth & $\mathrm{m}$ & not used & $1.000 \mathrm{E}+01$ & Not used \\
\hline Model: Nondispersion or Mass-Balance & - & not used & ND & Not used \\
\hline Well Pumping Rate & $\mathrm{m}^{3} / \mathrm{yr}$ & not used & $2.500 \mathrm{E}+02$ & Not used \\
\hline \multicolumn{5}{|c|}{ R015 Uncontaminated and Unsaturated Strata Hydrological Data } \\
\hline Number of Unsaturated Zone Strata & - & not used & 1 & Not used \\
\hline Thickness & $\mathrm{m}$ & not used & $4.000 \mathrm{E}+00$ & Not used \\
\hline Soil Density & $\mathrm{g} / \mathrm{cm}^{3}$ & not used & $1.500 \mathrm{E}+00$ & Not used \\
\hline Total Porosity & - & not used & $4.000 \mathrm{E}-01$ & Not used \\
\hline Effective Porosity & - & not used & 2.000E-01 & Not used \\
\hline Field Capacity & - & not used & $2.000 \mathrm{E}-01$ & Not used \\
\hline Soil-specific b Parameter & - & not used & $5.300 \mathrm{E}+00$ & Not used \\
\hline Hydraulic Conductivity & $\mathrm{m} / \mathrm{yr}$ & not used & $1.000 \mathrm{E}+01$ & Not used \\
\hline
\end{tabular}


Table A-1

RESRAD Parameters

(Page 4 of 6)

\begin{tabular}{|c|c|c|c|c|}
\hline Parameter & Units & CAU 476 & Defaults & Reference/Rationale \\
\hline \multicolumn{5}{|l|}{ R016 Distribution Coefficients and Leach Rates } \\
\hline Contaminated Zone $\mathrm{K}_{\mathrm{d}}$ (all Zones) & $\mathrm{cm}^{3} / \mathrm{g}$ & & & RESRAD Default \\
\hline Saturated Leach Rate & /yr & 0.0 & 0.0 & Not used \\
\hline Solubility Constant & - & 0.0 & 0.0 & Not used \\
\hline \multicolumn{5}{|l|}{ R017 Inhalation and External Gamma } \\
\hline Inhalation Rate & $\mathrm{m}^{3} / \mathrm{yr}$ & $1.230 \mathrm{E}+04$ & $8.400 \mathrm{E}+03$ & $\begin{array}{l}\text { RESRAD Default and for an individual } \\
\text { performing outdoor activities, a typical activity } \\
\text { mix can consist of } 37 \% \text { at a moderate activity } \\
\text { level, } 28 \% \text { at both resting and light activity } \\
\text { levels, and } 7 \% \text { at a heavy activity level, which } \\
\text { results in a } 1.4 \mathrm{~m}^{3} / \mathrm{h}\left(12,300 \mathrm{~m}^{3} / \mathrm{yr} \text { ) inhalation }\right. \\
\text { rate. (Yu, et al., } 1993)\end{array}$ \\
\hline Mass Loading for Inhalation & $\mathrm{g} / \mathrm{m}^{3}$ & $6.00 \mathrm{E}-04$ & 1E-04 & $\begin{array}{l}\text { The estimated mass loading for construction } \\
\text { activities. (Yu, et al., 1993) }\end{array}$ \\
\hline Exposure Duration & $\mathrm{yr}$ & 25 & 30 & Standard for Industrial/Commercial Scenario \\
\hline Shielding Factor Inhalation & - & 1.0 & 0.4 & Assumes no indoor time fraction \\
\hline Shielding Factor External Gamma & - & 1.0 & 0.7 & Assumes no indoor time fraction \\
\hline Fraction of Time Spent Indoors & - & 0.0 & 0.5 & Assumes no indoor time fraction \\
\hline Fraction of Time Spent Outdoors & - & 0.038 & 0.25 & $\begin{array}{l}\text { Scenario specific based on Industrial/ } \\
\text { Commercial Use Scenarios for standard } \\
\text { occupancy and low occupancy. }\end{array}$ \\
\hline Shape Factor & - & 1.0 & 1.0 & RESRAD Default \\
\hline \multicolumn{5}{|c|}{ R018 Ingestion Pathway Data, Dietary Parameters } \\
\hline Fruits, Vegetables, and Grain Consumption & $\mathrm{kg} / \mathrm{yr}$ & not used & $1.600 \mathrm{E}+02$ & Not used \\
\hline Leafy Vegetable Consumption & $\mathrm{kg} / \mathrm{yr}$ & not used & $1.400 \mathrm{E}+01$ & Not used \\
\hline Milk Consumption & $\mathrm{L} / \mathrm{yr}$ & not used & $9.200 \mathrm{E}+01$ & Not used \\
\hline Meat and Poultry Consumption & $\mathrm{kg} / \mathrm{yr}$ & not used & $6.300 \mathrm{E}+01$ & Not used \\
\hline
\end{tabular}


Table A-1

RESRAD Parameters

(Page 5 of 6)

\begin{tabular}{|c|c|c|c|c|}
\hline Parameter & Units & CAU 476 & Defaults & Reference/Rationale \\
\hline Fish Consumption & $\mathrm{kg} / \mathrm{yr}$ & not used & $5.400 \mathrm{E}+00$ & Not used \\
\hline Other Seafood Consumption & $\mathrm{kg} / \mathrm{yr}$ & not used & $9.000 \mathrm{E}-01$ & Not used \\
\hline Soil Ingestion Rate & $\mathrm{g} / \mathrm{yr}$ & $1.752 \mathrm{E}+02$ & 36.5 & EPA, 1991; 480 mg/day \\
\hline Drinking Water Intake & L/yr & not used & $5.100 \mathrm{E}+02$ & Not used \\
\hline Drinking Water Contaminated Fraction & - & not used & $1.000 \mathrm{E}+00$ & Not used \\
\hline Household Water Contaminated Fraction & - & not used & $1.000 \mathrm{E}+00$ & Not used \\
\hline Livestock Water Contaminated Fraction & - & not used & $1.000 E+00$ & Not used \\
\hline Irrigation Water Contaminated Fraction & - & not used & $1.000 \mathrm{E}+00$ & Not used \\
\hline Aquatic Food Contamination Fraction & - & not used & $5.000 \mathrm{E}-01$ & Not used \\
\hline Plant Food Contamination Fraction & - & not used & -1 & Not used \\
\hline Meat Contamination Fraction & - & not used & -1 & Not used \\
\hline Milk Contamination Fraction & - & not used & -1 & Not used \\
\hline \multicolumn{5}{|l|}{ R019 Ingestion Pathway Data, Nondietary } \\
\hline Livestock Fodder Intake for Meat & kg/day & not used & $6.800 E+01$ & Not used \\
\hline Livestock Fodder Intake for Milk & $\mathrm{kg} / \mathrm{day}$ & not used & $5.500 \mathrm{E}+01$ & Not used \\
\hline Livestock Water Intake for Meat & L/day & not used & $5.000 \mathrm{E}+01$ & Not used \\
\hline Livestock Water Intake for Milk & L/day & not used & $1.600 \mathrm{E}+02$ & Not used \\
\hline Livestock Soil Intake & $\mathrm{kg} / \mathrm{day}$ & not used & 5.000E-01 & Not used \\
\hline Mass Loading for Foliar Deposition & $\mathrm{g} / \mathrm{m}^{3}$ & not used & $1.000 \mathrm{E}-04$ & Not used \\
\hline Depth of Soil Mixing Layer & $\mathrm{m}$ & $1.500 \mathrm{E}-01$ & $1.500 \mathrm{E}-01$ & RESRAD Default \\
\hline Depth of Roots & $\mathrm{m}$ & not used & $9.000 \mathrm{E}-01$ & Not used \\
\hline
\end{tabular}


Table A-1

RESRAD Parameters

(Page 6 of 6)

\begin{tabular}{|c|c|c|c|c|}
\hline Parameter & Units & CAU 476 & Defaults & Reference/Rationale \\
\hline Drinking Water Fraction from Groundwater & - & not used & $1.000 \mathrm{E}+00$ & Not used \\
\hline $\begin{array}{l}\text { Household Water Fraction from } \\
\text { Groundwater }\end{array}$ & - & not used & $1.000 \mathrm{E}+00$ & Not used \\
\hline Livestock Water Fraction from Groundwater & - & not used & $1.000 \mathrm{E}+00$ & Not used \\
\hline Irrigation Fraction from Groundwater & - & not used & $1.000 \mathrm{E}+00$ & Not used \\
\hline \multicolumn{5}{|l|}{ R021 Radon } \\
\hline Radon Parameters Not Used & & \multicolumn{3}{|r|}{ Not used } \\
\hline $\begin{array}{l}\mathrm{cm}^{3} / \mathrm{g}=\text { Cubic centimeters per gram } \\
\mathrm{g} / \mathrm{cm}^{3}=\text { Grams per cubic centimeter } \\
\mathrm{g} / \mathrm{m}^{3}=\text { Grams per cubic meter } \\
\mathrm{g} / \mathrm{yr}=\text { Grams per year } \\
\mathrm{kg} / \mathrm{day}=\text { Kilograms per day } \\
\mathrm{kg} / \mathrm{yr}=\text { Kilograms per year } \\
\mathrm{L} / \mathrm{day}=\text { Liters per day } \\
\mathrm{L} / \mathrm{yr}=\text { Liters per year } \\
\mathrm{m}=\text { Meter } \\
\mathrm{m}^{2}=\text { Square meter }\end{array}$ & & \multicolumn{2}{|c|}{$\begin{array}{l}\mathrm{m} / \mathrm{sec}=\text { Meters per second } \\
\mathrm{m} / \mathrm{yr}=\text { Meters per year } \\
\mathrm{m}^{3} / \mathrm{h}=\text { Cubic meters per hour } \\
\mathrm{m}^{3} / \mathrm{yr}=\text { Cubic meters per year } \\
\mathrm{mg} / \mathrm{day}=\text { Milligrams per day } \\
\mathrm{mrem} / \mathrm{yr}=\text { Millirem per year } \\
\mathrm{ND}=\text { Nondetect } \\
\mathrm{pCi} / \mathrm{g}=\text { Picocuries per gram } \\
\text { RESRAD = Residual Radioactive } \\
\mathrm{yr}=\text { Year } \\
/ \mathrm{yr}=\text { Per year }\end{array}$} & \\
\hline
\end{tabular}




\section{Exhibit 1}

\section{RESRAD Summary Report: CAU 476 \\ (27 pages)}


Table of Contents

Part I: Mixture Sums and Single Radionuclide Guidelines

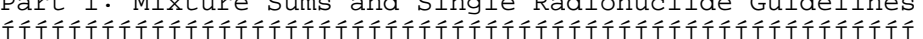

Dose Conversion Factor (and Related) Parameter Summary ...

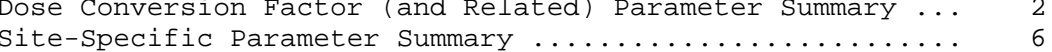
Summary of Pathway Selections ....................... 12 Contaminated Zone and Total Dose Summary ............... 13 Total Dose Components

Time $=0.000 \mathrm{E}+00$

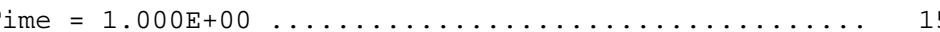

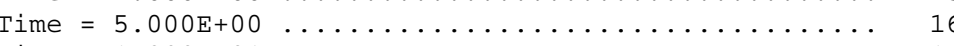

Time $=1.000 \mathrm{E}+01 \ldots \ldots \ldots \ldots \ldots \ldots \ldots \ldots \ldots \ldots \ldots \ldots \ldots, 17$

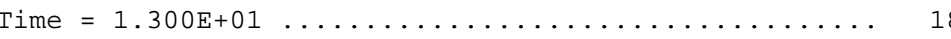

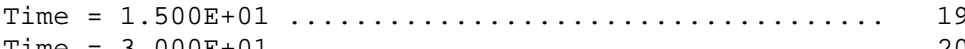

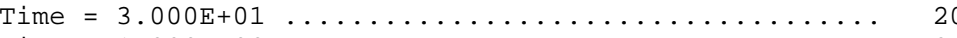

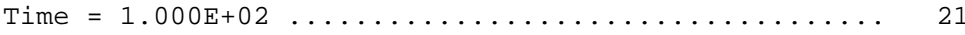

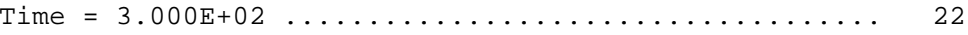

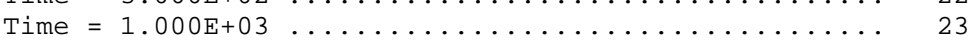

Dose/Source Ratios Summed Over All Pathways ........... 24

Single Radionuclide Soil Guidelines $\ldots \ldots \ldots \ldots \ldots \ldots \ldots . \ldots 24$

Dose Per Nuclide Summed over All Pathways ............ 26

Soil Concentration Per Nuclide $\ldots \ldots \ldots \ldots \ldots \ldots \ldots \ldots \ldots, 27$ 
Dose Conversion Factor (and Related) Parameter Summary File: FGR 13 MORBIDITY

$0 \quad 3$

Parameter

Current 3 Base ${ }_{3}^{3}$ Parameter

Menu 3

3 Current

Base

Name

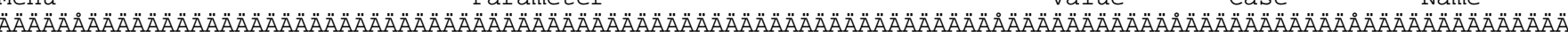

B-1 3 Dose conversion factors for inhalation, $\mathrm{mrem} / \mathrm{pCi}$

$B-1 \quad 3$ AC $-227+D$

$\mathrm{B}-1 \quad 3 \mathrm{Am}-241$

B-1 3 Co- 60

$\mathrm{B}-1 \quad 3 \mathrm{CS}-137+\mathrm{D}$

$\mathrm{B}-13 \mathrm{~Np}-237+\mathrm{D}$

$\mathrm{B}-1 \quad 3 \mathrm{~Pa}-231$

$\mathrm{B}-1 \quad 3 \mathrm{~Pb}-210+\mathrm{D}$

$\mathrm{B}-1 \quad 3 \mathrm{Pu}-238$

B-1 3 Pu-239

B-1 3 Ra-226+D

B-1 3 Sb-125+D

B-1 3 Sr $-90+D$

B-1 3 Th-229+D

B-1 3 Th -230

B-1 3 U-233

$B-1 \quad 3 \quad U-234$

B-1 3 U-235+D

D-1 3 Dose conversion factors for ingestion, $\mathrm{mrem} / \mathrm{pCi}$ :

D-1 3 AC-227+D

D-1 3 Am-241

D-1 3 Co-60

D-1 3 Cs $-137+D$

D-1 3 Np-237+D

D-1 3 Pa-231

D-1 3 Pb-210+D

D-1 3 Pu-238

$\mathrm{D}-1 \quad 3 \mathrm{Pu}-239$

D-1 3 Ra-226+D

D-1 3 Sb-125+D

D-1 3 Sr $-90+D$

D-1 3 Th-229+D

D-1 3 Th -230

D-1 3 U-233

D-1 3 U -234

D-1 3 U-235+D

D-34 3 Food transfer factors:

D-34 3 AC-227+D, plant/soil concentration ratio, dimensionless

$\mathrm{D}-34{ }^{3} \mathrm{AC}-227+\mathrm{D}$, , beef/livestock-intake ratio, (pCi/kg)/(pCi/d)

$\mathrm{D}-34{ }^{3} \mathrm{AC}-227+\mathrm{D}$, milk/livestock-intake ratio, $(\mathrm{pCi} / \mathrm{L}) /(\mathrm{pCi} / \mathrm{d})$

D-34 3

D-34 3 Am-241, plant/soil concentration ratio, dimensionless

D-34 3 Am-241

D-34 3 Am-241

D - 343

, beef/livestock-intake ratio, $(\mathrm{pCi} / \mathrm{kg}) /(\mathrm{pCi} / \mathrm{d})$

$\mathrm{milk} /$ livestock-intake ratio, $(\mathrm{pCi} / \mathrm{L}) /(\mathrm{pCi} / \mathrm{d})$

D-34 3 Co-60

D-34 3 Co-60

D -34 3 Co- 60

plant/soil concentration ratio, dimensionless

, beef/livestock-intake ratio, $(\mathrm{pCi} / \mathrm{kg}) /(\mathrm{pCi} / \mathrm{d})$

D-34 3

, milk/livestock-intake ratio, $(\mathrm{pCi} / \mathrm{L}) /(\mathrm{pCi} / \mathrm{d})$

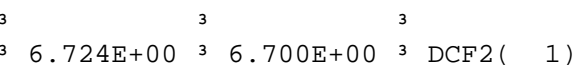

3 4.440E-01 3 4. 440E-01 3 DCF2( 2 (2)

2.190E-04 3 2.190E-04 3 DCF2( 3 )

3 3.190E-05 3 $3.190 \mathrm{E}-05$ 3 DCF2 ( 4$)$

$5.400 E-0130.400 E-013$ DCF2 ( 5$)$

$1.280 E+003$ 1.280E+00 3 DCF2( 5$)$

3 2.320E-02 3 1.360E-02 3 DCF2 ( 7$)$

$3.920 \mathrm{E}-01$ 3 3.920E-01 3 DCF2 ( 8)

4.290E-01 3 4.290E-01 3 DCF2( 10$)$

3 8.594E-03 3 8.580E-03 3 DCF2( 11$)$

$1.386 E-05$ 3 $1.220 E-05{ }^{3}$ DCF2( 12$)$

1.308E-03 3 1.300E-03 3 DCF2 ( 13$)$

$2.169 \mathrm{E}+00 \quad 3 \quad 2.150 \mathrm{E}+00 \quad 3 \mathrm{DCF} 2(14)$

3 3.260E-01 3 3.260E-01 3 DCF2( 15$)$

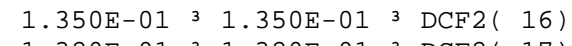

$1.320 \mathrm{E}-01{ }^{3} 1.320 \mathrm{E}-01{ }^{3}$ DCF2( 17$)$

$\begin{array}{l}3 \\ 1.230 E-01\end{array} 3$ 1.230E-01 3 DCF2( 18$)$

3

3 1.480E-02 3 1.410E-02 3 DCF3( 1$)$

$3.640 \mathrm{E}-03 \quad 3 \quad 3.640 \mathrm{E}-03 \quad 3 \mathrm{DCF} 3(2)$

$2.690 \mathrm{E}-05 \quad 3 \quad 2.690 \mathrm{E}-05 \quad 3$ DCF3( 3$)$

3 5.000E-05 3 5.000E-05 3 DCF3( 4)

4.444E-03 3 4.440E-03 3 DCF3( 5)

1.060E-02 3 $1.060 \mathrm{E}-02$ 3 DCF3( 6$)$

3 7.276E-03 3 5.370E-03 3 DCF3( 7)

$3.200 \mathrm{E}-03$ 3 $3.200 \mathrm{E}-03$ 3 DCF3( 8$)$

3 3.540E-03 3 3.540E-03 3 DCF3( 10$)$

3 1.321E-03 3 1.320E-03 3 DCF3( 11$)$

$3.647 \mathrm{E}-06$ 3 $2.810 \mathrm{E}-06$ 3 $\mathrm{DCF} 3(\mathrm{12})$

3 1.528E-04 3 1.420E- $04{ }^{3}$ DCF3( 13$)$

3 4.027E-03 3 3.530E-03 3 DCF3( 14)

5.480E-04 3 5.480E-04 3 DCF3( 15)

2.890E-04 3 2.890E-04 3 DCF3( 16$)$

2.830E- $044^{3}$ 2.830E- $04{ }^{3}$ DCF3( 17$)$

$2.673 E-04 \quad 3 \quad 2.660 E-04{ }^{3}$ DCF3( 18$)$

3

$2.500 \mathrm{E}-03 \quad 3 \quad 2.500 \mathrm{E}-03 \quad 3 \operatorname{RTF}(1,1)$

$2.000 \mathrm{E}-0532.000 \mathrm{3}-053 \operatorname{RTF}(1,2)$

2.000E-05 $32.000 \mathrm{E}-053 \operatorname{RTF}(1,3)$

1. $000 \mathrm{E}-03 \quad 3 \quad 1.000 \mathrm{E}-033 \operatorname{RTF}(2,1)$

3 5. 000E-05 3 5. 000E-05 3 RTF( 2,2$)$

2.000E-06 3 2.000E-06 $3 \operatorname{RTF}(2,3)$

8. $000 \mathrm{E}-02 \quad 3 \quad 8.000 \mathrm{E}-023 \operatorname{RTF}(3,1)$

3 2. $000 \mathrm{E}-02$ 3 2.000E-02 $3 \operatorname{RTF}(3,2)$

3 2.00९E-๑3 $32.000 \mathrm{E}-033 \operatorname{RTF}(3,3)$ 
Dose Conversion Factor (and Related) Parameter Summary (continued)

plant/soil concentration ratio, dimensionless $\mathrm{D}-343 \mathrm{~Np}-237+\mathrm{D}$, beef/livestock-intake ratio, $(\mathrm{pCi} / \mathrm{kg}) /(\mathrm{pCi} / \mathrm{d})$ $\mathrm{D}-343 \mathrm{~Np}-237+\mathrm{D}$, milk/livestock-intake ratio, ( $\mathrm{pCi} / \mathrm{L}) /(\mathrm{pCi} / \mathrm{d})$ $\mathrm{D}-344^{3}$

D-34 3 Pa-231

$\mathrm{D}-34{ }^{3} \mathrm{~Pa}-231$

D-34 3 Pa-231

D-34 3

D-34 $3 \mathrm{~Pb}-210+\mathrm{D}$

$\mathrm{D}-343 \mathrm{~Pb}-210+\mathrm{D}$

$\mathrm{D}-343 \mathrm{~Pb}-210+\mathrm{D}$

$\mathrm{D}-34$ 3 3 - 34 -

$\mathrm{D}-344^{3} \mathrm{Pu}-238$

D-34 3 Pu-238

D-34 3 Pu-238

D-34 3

D-34 3 Pu-239

D-34 3 Pu-239

D-34 3 Pu-239

D-34 3

D-34 3 Ra-226+D

$\mathrm{D}-34$ ( $\mathrm{Ra}-226+\mathrm{D}$

D-34 3 Ra-226+D

D-34 3

D-34 3 Sb-125+D

D-34 3 Sb-125+D

D-34 $3 \mathrm{Sb}-125+\mathrm{D}$

$D-34$ 3

D-34 3 Sr $-90+D$

$\mathrm{D}-34$ 3 $\mathrm{Sr}-90+\mathrm{D}$

D-34 3 Sr-90+D

D-34 3

D-34 3 Th-229+D

D-34 3 Th-229+D

milk/livestock-intake ratio, $(\mathrm{pCi} / \mathrm{L}) /(\mathrm{pCi} / \mathrm{d})$

plant/soil concentration ratio, dimensionless beef $/$ livestock-intake ratio, $(\mathrm{pCi} / \mathrm{kg}) /(\mathrm{pCi} / \mathrm{d})$
$\mathrm{milk} / \mathrm{livestock}$-intake ratio, $(\mathrm{pCi} / \mathrm{L}) /(\mathrm{pCi} / \mathrm{d})$

8. ๑०९E-๑3 3 8. $800 \mathrm{E}-03$ 3 $\operatorname{RTF}(4,3)$

3 2. $000 \mathrm{E}-02 \quad 3 \quad 2.000 \mathrm{E}-023 \operatorname{RTF}(5,1)$

3 1. $000 \mathrm{E}-03 \quad 3 \quad 1.000 \mathrm{E}-03 \quad 3 \operatorname{RTF}(5,2)$

5.

1. $000 \mathrm{E}-02 \quad 3 \quad 1.000 \mathrm{E}-023 \operatorname{RTF}(6,1)$

5.000E-03 3 5.000E-03 $3 \operatorname{RTF}(6,2)$

5. $000 \mathrm{E}-06$ 3 5. $3000 \mathrm{E}-06{ }^{3} \operatorname{RTF}(6,3)$

plant/soil concentration ratio, dimensionless beef/livestock-intake ratio, $(\mathrm{pCi} / \mathrm{kg}) /(\mathrm{pCi} / \mathrm{d})$ milk/livestock-intake ratio, $(\mathrm{pCi} / \mathrm{L}) /(\mathrm{pCi} / \mathrm{d})$

plant/soil concentration ratio, dimensionless beef/livestock-intake ratio, $(\mathrm{pCi} / \mathrm{kg}) /(\mathrm{pCi} / \mathrm{d})$ milk/livestock-intake ratio, $(\mathrm{pCi} / \mathrm{L}) /(\mathrm{pCi} / \mathrm{d})$

, plant/soil concentration ratio, dimensionless beef/livestock-intake ratio, $(\mathrm{pCi} / \mathrm{kg}) /(\mathrm{pCi} / \mathrm{d})$ $\mathrm{milk} /$ livestock-intake ratio, $(\mathrm{pCi} / \mathrm{L}) /(\mathrm{pCi} / \mathrm{d})$

plant/soil concentration ratio, dimensionless beef/livestock-intake ratio, $(\mathrm{pCi} / \mathrm{kg}) /(\mathrm{pCi} / \mathrm{d})$ $\mathrm{milk} /$ livestock-intake ratio, $(\mathrm{pCi} / \mathrm{L}) /(\mathrm{pCi} / \mathrm{d})$

plant/soil concentration ratio, dimensionless beef/livestock-intake ratio, $(\mathrm{pCi} / \mathrm{kg}) /(\mathrm{pCi} / \mathrm{d})$ milk/livestock-intake ratio, $(\mathrm{pCi} / \mathrm{L}) /(\mathrm{pCi} / \mathrm{d})$

, plant/soil concentration ratio, dimensionless beef/livestock-intake ratio, $(\mathrm{pCi} / \mathrm{kg}) /(\mathrm{pCi} / \mathrm{d})$ $\mathrm{milk} /$ livestock-intake ratio, $(\mathrm{pCi} / \mathrm{L}) /(\mathrm{pCi} / \mathrm{d})$

1.000E-02 3 1.000E-02 $3 \operatorname{RTF}(7,1)$

3 8. $000 \mathrm{E}-04$ 3 8.000E-04 3 RTF $(7,2)$

3 3. 000E-04 3 3.000E-04 $3 \operatorname{RTF}(7,3)$

1. $000 \mathrm{E}-03 \quad 3 \quad 1.000 \mathrm{E}-03 \quad 3 \operatorname{RTF}(8,1)$

1.000E-04 3 1.000E-04 $3 \operatorname{RTF}(8,2)$

1. $1.000 \mathrm{E}-06$ 3 $1.000 \mathrm{E}-063 \operatorname{RTF}(8,3)$

31 1. $000 \mathrm{E}-03 \quad 3 \quad 1.000 \mathrm{E}-033 \operatorname{RTF}(10,1)$

$1.000 \mathrm{E}-04$ 3 1 1. $000 \mathrm{E}-04 \quad 3 \operatorname{RTF}(10,2)$

3 $1.000 \mathrm{E}-06$ 3 $1.000 \mathrm{E}-063 \operatorname{RTF}(10,3)$

$3 \quad 3$

4. $000 \mathrm{E}-02$ 3 $4.000 \mathrm{E}-02$ 3 $\operatorname{RTF}(11,1)$

3 1. $000 \mathrm{E}-03$ 3 $1.000 \mathrm{E}-033 \operatorname{RTF}(11,2)$

$31.000 \mathrm{E}-03$ 3 $1.000 \mathrm{E}-03$ 3 $\operatorname{RTF}(11,3)$

$3 \quad 1.000 \mathrm{E}-02$ 3 $1.000 \mathrm{E}-023 \operatorname{RTF}(12,1)$

1. $000 \mathrm{E}-03$ 3 $1.000 \mathrm{E}-03$ 3 RTF $(12,2)$

3 1. 000E-04 3 1.000E-04 $3 \operatorname{RTF}(12,3)$

3 3

$3 \quad 3.000 \mathrm{E}-0133.000 \mathrm{~B}-013 \operatorname{RTF}(13,1)$

3 8.000E-๑3 3 8.000E-03 3 $\operatorname{RTF}(13,2)$

$32.000 \mathrm{E}-03$ 3 $2.000 \mathrm{E}-03 \quad 3 \operatorname{RTF}(13,3)$

plant/soil concentration ratio, dimensionless beef/livestock-intake ratio, ( $\mathrm{pCi} / \mathrm{kg}) /(\mathrm{pCi} / \mathrm{d})$

D -343 Th-229+D

D-34 3

D-34 3 Th -230

D-34 3 Th -230

D-34 3 Th-230

$\mathrm{D}-34{ }^{3}$

D- 34 3 U-233

$\mathrm{D}-34 \quad 3 \quad \mathrm{U}-233$

$\mathrm{D}-34 \quad 3 \quad \mathrm{U}-233$

$\mathrm{D}-344^{3}$

D- 34 3 U-234

D $-34 \quad 3 \quad \mathrm{U}-234$

$\mathrm{D}-34 \quad 3 \quad \mathrm{U}-234$

, plant/soil concentration ratio, dimensionless beef/livestock-intake ratio, ( $\mathrm{pCi} / \mathrm{kg}) /(\mathrm{pCi} / \mathrm{d})$ milk/livestock-intake ratio, $(\mathrm{pCi} / \mathrm{L}) /(\mathrm{pCi} / \mathrm{d})$

3 1. $000 \mathrm{E}-03 \quad 3$ 1.000E-03 $3 \operatorname{RTF}(14,1)$ 1. $000 \mathrm{E}-04 \quad 3 \quad 1.000 \mathrm{E}-043 \mathrm{RTF}(14,2)$ 3 5. 000E-06 3 5.000E-06 $3 \operatorname{RTF}(14,3)$

3 1. 000E-03 3 1. $1.00 \mathrm{E}-033 \operatorname{RTF}(15,1)$ 3 1. $000 \mathrm{E}-04$ 3 $1.000 \mathrm{E}-043 \operatorname{RTF}(15,2)$ 5.000E-06 3 5.000E-06 $3 \operatorname{RTF}(15,3)$

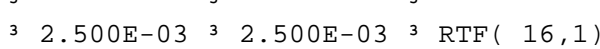
plant/soil concentration ratio, dimensionless beef/livestock-intake ratio, $(\mathrm{pCi} / \mathrm{kg}) /(\mathrm{pCi} / \mathrm{d})$ $\mathrm{milk} /$ livestock-intake ratio, $(\mathrm{pCi} / \mathrm{L}) /(\mathrm{pCi} / \mathrm{d})$

$3.400 \mathrm{E}-044^{3} \quad 3.400 \mathrm{E}-04{ }^{3} \operatorname{RTF}(16,2)$

3 6. ๑०९E-๑4 3 6. $6000 \mathrm{E}-044^{3} \operatorname{RTF}(16,3)$

, plant/soil concentration ratio, dimensionless beef/livestock-intake ratio, $(\mathrm{pCi} / \mathrm{kg}) /(\mathrm{pCi} / \mathrm{d})$

2.500E-03 $3 \quad 2.500 \mathrm{E}-03 \quad 3 \operatorname{RTF}(17,1)$

$3.400 \mathrm{E}-04 \quad 3 \quad 3.400 \mathrm{E}-04$ 3 $\operatorname{RTF}(17,2)$

, milk/livestock-intake ratio, (pCi/L)/(pCi/d) $36.000 \mathrm{E}-044^{3} 6.000 \mathrm{E}-04{ }^{3} \mathrm{RTF}(17,3)$ 
Dose Conversion Factor (and Related) Parameter Summary (continued)

$0_{\text {Menu }}^{3}{ }^{3}$ File: FGR 13 MORBIDITY

Menu 3

Parameter

3 Current ${ }^{3}$ Base ${ }_{3}^{3}$ Parameter

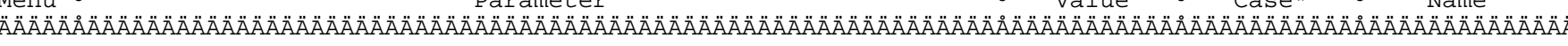

$\mathrm{D}-343 \mathrm{U}-235+\mathrm{D}$, plant/soil concentration ratio, dimensionless $32.500 \mathrm{E}-03 \quad 3 \quad 2.500 \mathrm{E}-03 \quad 3$ RTF( 18,1$)$

$\mathrm{D}-344^{3} \mathrm{U}-235+\mathrm{D}$, beef/livestock-intake ratio, (pCi/kg)/(pCi/d) $33.400 \mathrm{E}-043^{3} 3.400 \mathrm{E}-043 \mathrm{RTF}(18,2)$

$\mathrm{D}-34{ }^{3} \mathrm{U}-235+\mathrm{D}$, milk/livestock-intake ratio, (pCi/L)/(pCi/d) 3 6.000E-04 3 6.000E-04 3 RTF( 18,3)

D-5 3 Bioaccumulation factors, fresh water, L/kg:

D-5 3 AC-227+D, fish

D-5 3 AC-227+D, crustacea and mollusks

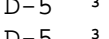

D-5 3 Am-241, fish

D-5 3 Am-241 ', crustacea and mollusks

D-5 3

D-5 ${ }^{3}$ Co-60, fish

D-5 3 Co-60, crustacea and mollusks

D $-5 \quad 3$

D-5 3 Cs $-137+D$ fish

D-5 3 Cs $-137+D$, crustacea and mollusks

D-5 3

$\mathrm{D}-5 \quad 3 \mathrm{~Np}-237+\mathrm{D}$, fish

D-5 $3 \mathrm{~Np}-237+\mathrm{D}$, crustacea and mollusks

D $-5 \quad 3$

D-5 3 Pa-231, fish

D-5 3 Pa-231, crustacea and mollusks

$D-5 \quad 3$

D-5 $3 \mathrm{~Pb}-210+\mathrm{D}$, fish

D-5 $3 \mathrm{~Pb}-210+\mathrm{D}$, crustacea and mollusks

D-5 3 Pu-238, fish

D-5 3 Pu-238, crustacea and mollusks

D-5 3 Pu-239, fish

D-5 3 Pu-239, crustacea and mollusks

D- 5 3

$D-5$

$\begin{array}{llll}D-5 & 3 & R a-226+D ， \text { fish } \\ \text { D-5 } & 3 & \text { Ra-226+D , crustacea and mollusks }\end{array}$

D -5 3

D-5 $3 \mathrm{Sb}-125+\mathrm{D}$, fish

D-5 3 Sb-125+D, crustacea and mollusks

D-5 3

D-5 3 Sr-90+D, fish

D-5 3 Sr-90+D, crustacea and mollusks

D- 5

D-5 3 Th-229+D, fish

D-5 3 Th-229+D , crustacea and mollusks

D-5 3 Th-230 , fish

D-5 3 Th-230, crustacea and mollusks

D-5 3

D-5 3 U-233 , fish

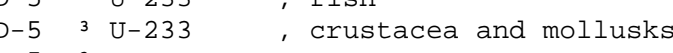

\begin{tabular}{|c|c|c|c|}
\hline & & 3 & \\
\hline & 3 & 3 & \\
\hline $1.500 \mathrm{E}+01$ & $31.500 \mathrm{E}+01$ & 3 BIOFAC ( & 1,1 \\
\hline 1. $000 \mathrm{E}+03$ & $\begin{array}{ll}3 & 1.000 \mathrm{E}+03 \\
3 & \end{array}$ & ${ }_{3}^{3}$ BIOFAC & $1,2)$ \\
\hline $3 . \odot \odot \odot E+\odot 1$ & $\begin{array}{l}3 \\
3\end{array} 3.00 \odot \mathrm{E}+01$ & 3 BIOFAC ( & \\
\hline 1. $000 \mathrm{E}+03$ & 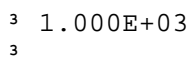 & $\begin{array}{l}3 \\
3\end{array}$ & \\
\hline 3. $\odot \odot \odot E+\odot 2$ & $3 \quad 3.00 \odot E+\odot 2$ & 3 BIOFAC( & \\
\hline 2. $\odot \odot \odot E+\odot 2$ & $\begin{array}{ll}3 & 2.000 \mathrm{E}+\odot 2 \\
3 & \end{array}$ & ${ }_{3}^{3}$ BIOFAC ( & \\
\hline 2. $\odot \odot \odot E+\odot 3$ & 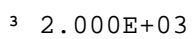 & 3 BIOFAC( & \\
\hline 1. $\odot \odot ० E+\odot 2$ & $\begin{array}{ll}3 & 1.000 \mathrm{E}+02 \\
3 & \end{array}$ & ${ }_{3}^{3}$ BIOFAC ( & \\
\hline 3. $\odot \odot \odot E+\odot 1$ & $3 \quad 3.00 \odot E+01$ & 3 BIOFAC ( & \\
\hline $4.000 \mathrm{E}$ & $\begin{array}{ll}3 & 4.6 \\
3 & \end{array}$ & $\begin{array}{ll}3 & B I C \\
3 & \end{array}$ & \\
\hline 1. $0 \odot \odot E+\odot 1$ & $3 \quad 1.0 \odot \odot E+\odot 1$ & 3 BIOFAC ( & \\
\hline $1.100 \mathrm{E}+02$ & $\begin{array}{ll}3 & 1.1 \\
3 & \end{array}$ & $\begin{array}{l}3 \text { BIOFAC ( } \\
3\end{array}$ & \\
\hline 3. $\odot \odot \odot E+\odot 2$ & $\begin{array}{l}3 \\
3\end{array} 3.0 \odot \odot \mathrm{E}+\odot 2$ & $3 \mathrm{BI}$ & \\
\hline 1. $000 \mathrm{E}+02$ & $\begin{array}{ll}3 & 1.0 \\
3 & \end{array}$ & $\begin{array}{l}3 \\
3\end{array}$ BIOFAC ( & \\
\hline 3. $00 \odot E+01$ & $\begin{array}{l}3 \\
3\end{array} 3.000 \mathrm{E}+01$ & $3 \mathrm{BI}$ & \\
\hline 1. $000 \mathrm{E}+02$ & & & \\
\hline $3.000 \mathrm{E}+01$ & 33.0 & $3 \mathrm{BI}$ & \\
\hline 1. $.000 \mathrm{E}+02$ & & & \\
\hline $5.000 \mathrm{E}+01$ & 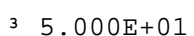 & $3 \mathrm{BI}$ & \\
\hline $2.500 \mathrm{E}+02$ & & 3 BIOFAC ( & \\
\hline 1. $\odot \odot \odot E+\odot 2$ & $3 \quad 1.0 \odot \odot E+\odot 2$ & $3 \mathrm{BI}$ & \\
\hline & & 3 BIOFAC & \\
\hline & $36.000 \mathrm{E}+01$ & $3 \mathrm{BI}$ & \\
\hline & & 3 BIOFAC & \\
\hline & $\begin{array}{ll}3 & 1.0\end{array}$ & $3 \mathrm{BIO}$ & \\
\hline $5.000 \mathrm{E}+02$ & 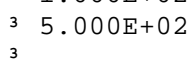 & & \\
\hline$\partial E+02$ & 31.0 & $3 \mathrm{BI}$ & \\
\hline 5. $000 \mathrm{E}+02$ & $\begin{array}{ll}3 & 5.000 \mathrm{E}+\end{array}$ & 3 BIOFAC & \\
\hline & 31. & $3 \mathrm{~B}$ & \\
\hline & & $3 \mathrm{~B}$ & \\
\hline
\end{tabular}



1RESRAD, Version 6.3
T $\ll$ Limit $=180$ days
01/30/2007 15:00 Page 5
Summary : CAU 476
File: CAU 476. RAD

Dose Conversion Factor (and Related) Parameter Summary (continued)

Menu ${ }^{3}$ File: FGR 13 MORBIDITY

Parameter

3 Current ${ }^{3}$ Base ${ }^{3}$ Parameter

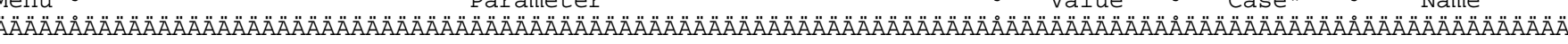

D-5 3 U-234 , fish $31.000 \mathrm{E}+0131.000 \mathrm{E}+013 \mathrm{BIOFAC}(17,1)$

D-5 3 U-234, crustacea and mollusks $36.000 \mathrm{E}+013$ 6.000E+01 3 BIOFAC( 17,2)

D -5 - 3

D-5 3 U-235+D , fish

3

6.

D-5 3 U-235+D, crustacea and mollusks

$1.000 \mathrm{E}+011^{3} 1.000 \mathrm{E}+01{ }^{3} \operatorname{BIOFAC}(18,1)$

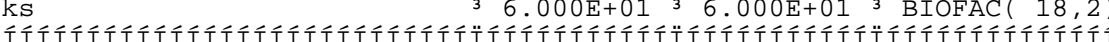

*Base Case means Default. Lib W/o Associate Nuclide contributions. 
Site-Specific Parameter Summary Menu 3

Menu 3 Parameter $\quad 3$ Input 3 Default 3 (If different from user input) 3 Name

Menu 3 Parameter 3 Input 3 Default 3 (If different from user input) 3 Name

Used by RESRAD 3 Parameter

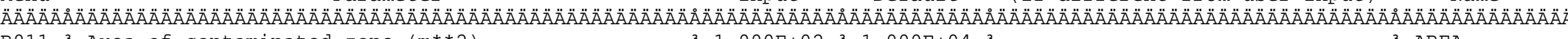
R011 3 Area of contaminated zone $(\mathrm{m} * * 2)$

R011 3 Thickness of contaminated zone $(\mathrm{m})$

R011 3 Length parallel to aquifer flow $(\mathrm{m})$

R011 3 Basic radiation dose limit (mrem/yr)

R011 3 Time since placement of material (yr)

R011 3 Times for calculations ( $\mathrm{yr}$ )

R011 3 Times for calculations ( $y r$ )

R011 3 Times for calculations ( $\mathrm{yr}$ )

R011 3 Times for calculations (yr)

R011 3 Times for calculations $(y r)$

R011 3 Times for calculations $(y r)$

R011 3 Times for calculations ( $y r$ )

R011 3 Times for calculations $(y r)$

R011 3 Times for calculations ( $y r$ )

$$
3
$$

R012 3 Initial principal radionuclide ( $\mathrm{pCi} / \mathrm{g}$ ): R012 3 Initial principal radionuclide ( $\mathrm{pCi} / \mathrm{g})$ : R012 3 Initial principal radionuclide ( $\mathrm{pCi} / \mathrm{g}$ ): $\mathrm{R} 0123$ Initial principal radionuclide $(\mathrm{pCi} / \mathrm{g})$ : R012 3 Initial principal radionuclide ( $\mathrm{pCi} / \mathrm{g}$ ): $\mathrm{R} 0123$ Initial principal radionuclide ( $\mathrm{pCi} / \mathrm{g})$ R012 3 Initial principal radionuclide ( $\mathrm{pCi} / \mathrm{g})$ : R012 3 Concentration in groundwater ( $\mathrm{pCi} / \mathrm{L})$ : R012 3 Concentration in groundwater $(\mathrm{pCi} / \mathrm{L})$ : R012 3 Concentration in groundwater ( $\mathrm{pCi} / \mathrm{L})$ : R012 3 Concentration in groundwater $(\mathrm{pCi} / \mathrm{L}): \quad \mathrm{Pu}-238$ $(\mathrm{pCi} / \mathrm{L}): \mathrm{Pu}-239$ $1.000 \mathrm{E}+02{ }^{3} 1.000 \mathrm{E}+04$ $1.500 \mathrm{E}-01 \quad 3 \quad 2.000 \mathrm{E}+00$ not used 3 1. $1.000 \mathrm{E}+02$ $2.500 \mathrm{E}+011^{3} \quad 3.000 \mathrm{E}+01$ $0.000 \mathrm{E}+00 \quad 3 \quad 0.000 \mathrm{E}+00$ $1.000 \mathrm{E}+00{ }^{3} \quad 1.000 \mathrm{E}+00$

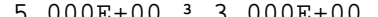
1. $000 E+01$ 3 $1.000 E+01$ $1.300 E+01$ 3. $3.000 E+01$ $1.500 \mathrm{E}+0131.00$. $3.000 \mathrm{E}+01$ 3 $3.000 \mathrm{E}+02$ $1.000 \mathrm{E}+02$ 3 $1.000 \mathrm{E}+03$ 3. $000 \mathrm{E}+02 \quad 3 \quad 0.000 \mathrm{E}+00$ $1.000 \mathrm{E}+03$ 3 $0.000 \mathrm{E}+003$ $A m-241$ Co- 60 $\mathrm{Cs}-137$
$\mathrm{Pu}-238$ $\mathrm{Pu}-239$ $\mathrm{Sb}-125$ Sr -90 Am-241 Co-60 Cs -137 $\mathrm{Pu}-239$
$\mathrm{Sb}-125$ $(\mathrm{pCi} / \mathrm{L}): \mathrm{Sr}-90$

R013 3 Cover depth (m)

R013 3 Density of cover material $\left(\mathrm{g} / \mathrm{cm}^{* *} 3\right)$

R013 3 Cover depth erosion rate (m/yr)

R013 3 Density of contaminated zone $\left(\mathrm{g} / \mathrm{cm}^{* *} 3\right)$

R013 3 Contaminated zone erosion rate $(\mathrm{m} / \mathrm{yr})$

R013 3 Contaminated zone total porosity

R013 3 Contaminated zone field capacity

R013 3 Contaminated zone hydraulic conductivity (m/yr) R013 3 Contaminated zone b parameter

R013 3 Average annual wind speed $(\mathrm{m} / \mathrm{sec})$

R013 3 Humidity in air $\left(\mathrm{g} / \mathrm{m}^{* \star} 3\right)$

R013 3 Evapotranspiration coefficient

R013 3 Precipitation (m/yr)

R013 3 Irrigation ( $\mathrm{m} / \mathrm{yr}$ )

R013 3 Irrigation mode

R013 3 Runoff coefficient

R013 3 Watershed area for nearby stream or pond $\left(m^{* *} 2\right)$

R013 3 Accuracy for water/soil computations

R014 3 Density of saturated zone $\left(\mathrm{g} / \mathrm{cm}^{* *} 3\right)$

R014 3 Saturated zone total porosity
$32.80 \odot \mathrm{E}+\odot \odot \quad 3 \quad \odot, 0 \odot \odot \mathrm{E}+\Theta \odot$

$1.760 \mathrm{E}+0 \odot \quad 3 \quad 0.000 \mathrm{E}+0 \Theta$

$3.820 \mathrm{E}+02 \quad 3 \quad 0.000 \mathrm{E}+00$

$1.030 \mathrm{E}+0 \odot$ 3 $\odot .000 \mathrm{E}+0 \odot$

$9.600 \mathrm{E}+\Theta \odot{ }^{3} \quad 0.000 \mathrm{E}+\odot \odot$

$3.000 \mathrm{E}+0 \odot$ अ $\quad 0.000 \mathrm{E}+0 \odot$

$1.300 \mathrm{E}+01$ 3 $0.000 \mathrm{E}+00$

not used $3 \quad 0.000 \mathrm{E}+0 \theta^{3}$

not used $30.000 E+00$

3 not used $30.000 E+0 \theta^{3}$

not used 3 O. OOEE

not used 30.000 COO

not used $3 \quad 0.000 \mathrm{E}+00$

not used $30.000 \mathrm{E}+00$

उ $. .0 \odot \odot \mathrm{E}+\odot \odot \quad 3 \quad 0.00 \odot \mathrm{E}+\odot \odot$

not used $31.500 \mathrm{E}+00^{3}$

not used $31.000 \mathrm{E}-03$

$1.500 \mathrm{E}+00 \quad 3 \quad 1.500 \mathrm{E}+0 \Theta^{3}$

$1.000 \mathrm{E}-03$ 3 $1.000 \mathrm{E}-03$

4. $000 \mathrm{E}-013$ 4. 3 . $00 \mathrm{E}-01$

2. $000 \mathrm{E}-01 \quad 3 \quad 2.000 \mathrm{E}-01$

$1.000 \mathrm{E}+01$ 3 $1.000 \mathrm{E}+01$

$5.300 \mathrm{E}+00 \quad 3 \quad 5.300 \mathrm{E}+00$

$5.170 \mathrm{E}+00$ 3 $2.000 \mathrm{E}+0 \odot$

not used $3 \quad 8.000 \mathrm{E}+000^{3}$

5. $000 \mathrm{E}-01 \quad 3 \quad 5.000 \mathrm{E}-01$

3. $045 \mathrm{E}-01$ 3 $1.000 \mathrm{E}+00$

$0.000 \mathrm{E}+003 \quad 2.000 \mathrm{E}-01$

overhead 3 overhead

4.000E-01 3 2.000E-01

not used $31.000 E+06$

not used $31.000 \mathrm{E}-03$

not used 3 1.500E+00

3 not used 3 4.00०E-01
- -

3 THICKO

$3 \mathrm{~T}(2)$

3 T( 3$)$

$3 \mathrm{~T}(4)$

3 T( 5$)$

$3 \mathrm{~T}(6)$

$3 \mathrm{~T}(7)$

3 T $(8)$
3 T $(9)$

S1 ( 2)

$3 \mathrm{~S} 1(3)$

3 S1 ( 4)

3 S1 ( 8)

3 S1(10)

3
3 $1(12)$

3 W1 ( 2)

3 W1 (3)

3 W1 ( 4)

3 W1 ( 8)

3 W1(10)

3 W1 12$)$

3 COVERO

3 DENSCV

3 VCV

3 DENSCZ

$3 \mathrm{VCZ}$

3 TPCZ

3 FCCZ

$3 \mathrm{HCCZ}$

$3 \mathrm{BCZ}$

3 WIND

3 HUMID

3 EVAPTR

3 PRECIP

3 RI

3 IDITCH

3 RUNOFF

3 WAREA

3 EPS

3 DENSAQ

3 TPSZ 

Site-Specific Parameter Summary (continued)

Menu 3

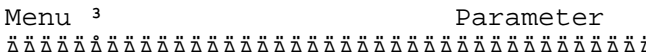

3 Input 3 Default 3 (If different from user

Used by RESRAD 3 Parameter

R014 3 Saturated zone effective porosity

R014 3 Saturated zone effective porosity_ not used $32.000 \mathrm{r}-011^{3}$

R014 Saturated zone field capacity

R014 3 Saturated zone hydraulic conductivity $(\mathrm{m} / \mathrm{yr}) \quad 3$ not used $31.000 \mathrm{E}+02$

R014 3 Saturated zone hydraulic gradient

not used $32.000 \mathrm{E}-02$

R014 3 Saturated zone b parameter

R014 3 Well pump intake depth (m below water table)

R014 3 Model: Nondispersion (ND) or Mass-Balance (MB)

R014 3 Well pumping rate $\left(m^{* *} 3 / \mathrm{yr}\right)$

R015 3 Number of unsaturated zone strata

R015 3 Unsat. zone 1, thickness (m)

R015 3 Unsat. zone 1 , soil density $\left(\mathrm{g} / \mathrm{cm}^{* *} 3\right)$

R015 3 Unsat. zone 1, total porosity

R015 3 Unsat. zone 1, effective porosity

R015 3 Unsat. zone 1, field capacity

R015 3 Unsat. zone 1, soil-specific b parameter

R015 3 Unsat. zone 1 , hydraulic conductivity (m/yr)

not used $35.300 \mathrm{E}+00^{3}$

not used $31.000 \mathrm{E}-03$

not used 3 1.000E+01

not used 3 ND

not used $32.500 \mathrm{E}+02$

not

not used 3

not used 3 4. $4000 \mathrm{E}+00^{3}$

not used 3 1.500E $+0 \odot$

not used 3 4.000E-01

3 not used 32 2.000E-01 3

3 not used 3 2.000E-01

3 not used 3 5.300E+00 3

not used $31.000 \mathrm{E}+01$

Distribution coefficients for Am-241

R016 3 Contaminated zone $\left(\mathrm{cm}^{* *} 3 / \mathrm{g}\right)$

R016 3 Unsaturated zone $1\left(\mathrm{~cm}^{* *} 3 / \mathrm{g}\right)$

R016 3 Saturated zone $\left(\mathrm{cm}^{* *} 3 / \mathrm{g}\right)$

$\mathrm{R} 016$ Leach rate (/yr)

R016 3 Solubility constant

R016 3 Distribution coefficients for Co-60

R016 3 Contaminated zone $\left(\mathrm{cm}^{* *} 3 / \mathrm{g}\right)$

R016 3 Unsaturated zone $1\left(\mathrm{~cm}^{* *} 3 / \mathrm{g}\right)$

R016 3 Saturated zone $\left(\mathrm{cm}^{* *} 3 / \mathrm{g}\right)$

R016 3 Leach rate (/yr)

R016 3 Solubility constant

R016 3 Distribution coefficients for Cs-137

R016 3 Contaminated zone $\left(\mathrm{cm}^{* *} 3 / \mathrm{g}\right)$

R016 3 Unsaturated zone $1(\mathrm{~cm} * * 3 / \mathrm{g})$

R016 3 Saturated zone $\left(\mathrm{cm}^{* *} 3 / \mathrm{g}\right)$

R016 3 Leach rate (/yr)

R016 3 Solubility constant

R016 3 Distribution coefficients for Pu-238

R016 3 Contaminated zone $\left(\mathrm{cm}^{* *} 3 / \mathrm{g}\right)$

R016 3 Unsaturated zone $1\left(\mathrm{~cm}^{* *} 3 / \mathrm{g}\right)$

R016 3 Saturated zone $\left(\mathrm{cm}^{* *} 3 / \mathrm{g}\right)$

R016 3 Leach rate (/yr)

RO16 3 Solubility constant

$2.000 \mathrm{E}+01 \quad 3 \quad 2.000 \mathrm{E}+01$

not used $32.000 E+01$

not used 32 . $000 \mathrm{E}+01$

$\odot .0 \odot \odot \mathrm{E}+\Theta \odot \quad 3 \quad 0.0 \odot \odot \mathrm{E}+\odot \odot \quad 3$

$0.000 \mathrm{E}+0 \odot \quad 3 \quad 0.000 \mathrm{E}+00^{3}$

$1.000 \mathrm{E}+03 \quad 3 \quad 1.000 \mathrm{E}+03$

not used $31.000 \mathrm{E}+03$

not used 3 1.000E+03

$0.000 \mathrm{E}+00{ }^{3} \quad 0.000 \mathrm{E}+00 \quad 3$

$\odot .000 \mathrm{E}+00 \quad 3 \quad 0.000 \mathrm{E}+0 \odot$

$4.600 \mathrm{E}+03 \quad 3 \quad 4.600 \mathrm{E}+03$

not used 3 4.600E+03

not used 3 4.600E+03

$0.000 \mathrm{E}+00 \quad 3 \quad 0.000 \mathrm{E}+00 \quad 3$

$0.000 \mathrm{E}+\odot \Theta^{3} \quad 0.000 \mathrm{E}+\Theta \odot \Theta^{3}$ 3

$2.000 \mathrm{E}+03 \quad 3 \quad 2.000 \mathrm{E}+03$

not used $32.000 \mathrm{E}+03$

not used $32.000 \mathrm{E}+03$

$30.000 E+0 \odot \quad 3 \quad 0.000 E+0 \odot$

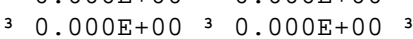

$\begin{array}{ll}3 & \text { EPSZ } \\ 3 & \text { FCSZ }\end{array}$

$\begin{array}{ll}3 & \text { FCSZ } \\ 3 & \text { HCSZ }\end{array}$

3 HGWT

3 BSZ

3 VWT

3 DWIBWT

MODEL

UW

3

3 NS

$3 \mathrm{H}(1)$

3 DENSUZ(1)

$3 \operatorname{TPUZ}(1)$

EPUZ (1)

FCUZ(1)

$\operatorname{BUZ}(1)$

$\operatorname{HCUZ}(1)$

DCNUCC ( 2)

3 DCNUCU $(2,1)$

DCNUCS( 2)

ALEACH ( 2)

SOLUBK( 2)

not used

- -

4. $059 \mathrm{E}-04$

not used

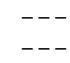

$--$

$8.826 \mathrm{E}-05$

not used

$\operatorname{DCNUCC}(3)$

$\operatorname{DCNUCU}(3,1)$

DCNUCS ( 3 )

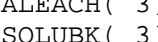

DCNUCC ( 4)

$\operatorname{DCNUCU}(4,1)$

DCNUCS ( 4)

3 ALEACH ( 4)

SOLUBK( 4$)$

3 DCNUCC ( 8)

$-$

$2.030 \mathrm{E}-04$

not used
3 DCNUCU $(8,1)$

3 DCNUCS ( 8)

3 ALEACH( 8$)$ 
Site-Specific Parameter Summary (continued)

$0_{\text {Menu }}^{3}{ }^{3}$

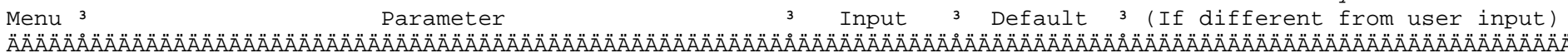

Used by RESRAD 3 Parameter

R016 3 Distribution coefficients for Pu-239

R016 3 Contaminated zone $\left(\mathrm{cm}^{* *} 3 / \mathrm{g}\right)$

R016 3 Unsaturated zone $1(\mathrm{~cm} * * 3 / \mathrm{g})$

R016 3 Saturated zone $\left(\mathrm{cm}^{* *} 3 / \mathrm{g}\right)$

R016 3 Leach rate (/yr)

R016 3 Solubility constant

R016 3 Distribution coefficients for Sb-125

R016 3 Contaminated zone $\left(\mathrm{cm}^{* *} 3 / \mathrm{g}\right)$

R016 3 Unsaturated zone $1\left(\mathrm{~cm}^{* *} 3 / \mathrm{g}\right)$

R016 3 Saturated zone $\left(\mathrm{cm}^{* *} 3 / \mathrm{g}\right)$

R016 3 Leach rate (/yr)

R016 ${ }^{3}$ Solubility constant

R016 3 Distribution coefficients for $\mathrm{Sr}-90$

R016 3 Contaminated zone $\left(\mathrm{cm}^{* *} 3 / \mathrm{g}\right)$

R016 3 Unsaturated zone $1\left(\mathrm{~cm}^{* *} 3 / \mathrm{g}\right)$

R016 3 Saturated zone $\left(\mathrm{cm}^{* *} 3 / \mathrm{g}\right)$

R016 3 Leach rate (/yr)

R016 3 Solubility constant

R016 3 Distribution coefficients for daughter AC-227

R016 3 Contaminated zone $\left(\mathrm{cm}^{* *} 3 / \mathrm{g}\right)$

R016 3 Unsaturated zone $1\left(\mathrm{~cm}^{* *} 3 / \mathrm{g}\right)$

R016 3 Saturated zone $\left(\mathrm{cm}^{* *} 3 / \mathrm{g}\right)$

R016 3 Leach rate (/yr)

R016 3 Solubility constant

R016 3 Distribution coefficients for daughter $\mathrm{Np}-237$

R016 3 Contaminated zone $\left(\mathrm{cm}^{* *} 3 / \mathrm{g}\right)$

R016 3 Unsaturated zone $1\left(\mathrm{~cm}^{* *} 3 / \mathrm{g}\right)$

R016 3 Saturated zone $\left(\mathrm{cm}^{* *} 3 / \mathrm{g}\right)$

R016 3 Leach rate (/yr)

R016 3 Solubility constant

R016 3 Distribution coefficients for daughter Pa-231

R016 3 Contaminated zone $\left(\mathrm{cm}^{* *} 3 / \mathrm{g}\right)$

R016 3 Unsaturated zone $1\left(\mathrm{~cm}^{* *} 3 / \mathrm{g}\right)$

R016 3 Saturated zone $\left(\mathrm{cm}^{* *} 3 / \mathrm{g}\right)$

R016 3 Leach rate (/yr)

R016 3 Solubility constant

R016 3 Distribution coefficients for daughter $\mathrm{Pb}-210$

R016 3 Contaminated zone $\left(\mathrm{cm}^{* *} 3 / \mathrm{g}\right)$

R016 3 Unsaturated zone $1\left(\mathrm{~cm}^{* *} 3 / \mathrm{g}\right)$

R016 3 Saturated zone $\left(\mathrm{cm}^{* *} 3 / \mathrm{g}\right)$

R016 3 Leach rate (/yr)

$2.0 \odot \odot \mathrm{E}+03 \quad 3 \quad 2.000 \mathrm{E}+03$

$\begin{array}{lll}2.000 \mathrm{E}+03 & 3 & 2.000 \mathrm{E}+03 \\ \text { not used } & 3 & 2.000 \mathrm{E}+03\end{array}$

not used $32.000 \mathrm{E}+03$

$0.000 \mathrm{E}+0 \odot \quad 3 \quad 0.000 \mathrm{E}+0 \odot$

$\odot .000 \mathrm{E}+\odot \Theta^{3} \quad 0.000 \mathrm{E}+\Theta \odot{ }^{3}$

$0.000 \mathrm{E}+00 \quad 3 \quad 0.000 \mathrm{E}+00$

not used $30.000 \mathrm{E}+\odot \odot$

not used $30.000 \mathrm{E}+00^{3}$

$0.000 \mathrm{E}+00{ }^{3} \quad 0.000 \mathrm{E}+00$

$\odot .000 \mathrm{E}+0 \Theta^{3} \quad 0.000 \mathrm{E}+0 \odot^{3}$

3. $000 \mathrm{E}+01 \quad 3 \quad 3,000 \mathrm{E}+01$

not used 3 3. $30 \odot E+01$

not used 3 used 3 3. $3000 \mathrm{E}+01$

$\begin{array}{lll}\text { not used } & 3 & 3.00 \odot E+01 \\ 0.00 \odot E+0 \odot & 3 & 0.00 \odot E+0 \odot\end{array}$

$\odot .000 \mathrm{E}+0 \odot$ 3 $\odot .000 \mathrm{E}+\odot \odot$

2. $000 \mathrm{E}+01 \quad 3 \quad 2.000 \mathrm{E}+01$

not used 3 2.000E+01

not used 3

not used $2.000 \mathrm{E}+01$

$\odot .000 \mathrm{E}+00$ 3 $0.000 \mathrm{E}+0 \odot$

$\odot . \odot \odot \odot E+\odot \odot{ }^{3} \quad \odot . \odot \odot \odot E+\odot \odot$

$3-1.000 \mathrm{E}+00 \quad 3-1.000 \mathrm{E}+00$

not used $3-1.000 \mathrm{E}+00^{3}$

not used $3-1.0000^{3} 00^{3}$

O.

$0.000 \mathrm{E}+0 \odot{ }^{3} \quad 0.000 \mathrm{E}+\odot \odot^{3}$

$5.000 \mathrm{E}+0135.000 \mathrm{E}+01$

3 not used 3 5.000E+01 3

3 not used 3 5.000E +01

$0.000 \mathrm{E}+0 \mathrm{O}^{3} \quad 0.000 \mathrm{E}+0 \mathrm{O}^{3}$

$0.000 \mathrm{E}+0 \odot \quad 3 \quad 0.000 \mathrm{E}+0 \Theta^{3}$

3

$1.000 \mathrm{E}+02 \quad 3 \quad 1.000 \mathrm{E}+02$

not used 3 1.000E $+02^{3}$

3 not used 3 1.000E+०2

$3 \quad 0.000 \mathrm{E}+0 \odot \quad 3 \quad \odot .00 \odot \mathrm{E}+\odot \odot$

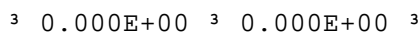

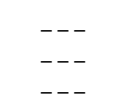

$--$

not used

$\ldots$

$-$

$2.150 \mathrm{E}+\odot \odot$

not used

- -

$--$

1. $345 \mathrm{E}-02$

not used

$--$

$-$

2. $011 \mathrm{E}-02$

not used

2. $574 \mathrm{E}+02$

-

$1.576 \mathrm{E}-03$

not used

$--$

$-$

8. $089 \mathrm{E}-03$

not used

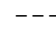

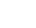

4. $052 \mathrm{E}-03$

not used
Name

ÄÄÄÄÄÄÄÄÄÄÄÄÄ

$\operatorname{DCNUCC}(10)$

$\operatorname{DCNUCU}(10,1)$

3 DCNUCS (10)

3 ALEACH (10)

SOLUBK (10)

DCNUCC (12)

$3 \operatorname{DCNUCU}(12,1)$

DCNUCS $(12)$

ALEACH (12)

SOLUBK(12)

DCNUCC (13)

$3 \operatorname{DCNUCU}(13,1)$

DCNUCS (13)

ALEACH (13)

SOLUBK (13)

$\operatorname{DCNUCC}(1)$

$\operatorname{DCNUCU}(1,1)$

3 DCNUCS( 1$)$

3 ALEACH ( 1$)$

SOLUBK( 1$)$

DCNUCC ( 5)

DCNUCU $(5,1)$

DCNUCS( 5 )

ALEACH ( 5 )

SOLUBK( 5 )

DCNUCC ( 6)

3 DCNUCU $(6,1)$

DCNUCS ( 6)

ALEACH ( 6)

SOLUBK( 6)

DCNUCC ( 7)

3 DCNUCU $(7,1)$

3 DCNUCS ( 7)

3 SOLUBK ( 7 ) 
Site-Specific Parameter Summary (continued)

$\odot_{\text {Menu }}{ }^{3}$

Parameter

User

Used by RESRAD

3 Parameter

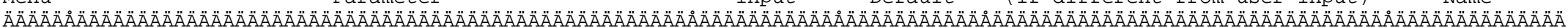

R016 3 Distribution coefficients for daughter Ra-226

R016 3 Contaminated zone $\left(\mathrm{cm}^{* *} 3 / \mathrm{g}\right)$

R016 3 Unsaturated zone $1\left(\mathrm{~cm}^{* *} 3 / \mathrm{g}\right)$

R016 3 Saturated zone $\left(\mathrm{cm}^{* *} 3 / \mathrm{g}\right)$

R016 3 Leach rate (/yr)

R016 3 Solubility constant

R016 3 Distribution coefficients for daughter Th-229

R016 3 Contaminated zone $\left(\mathrm{cm}^{* *} 3 / \mathrm{g}\right)$

R016 3 Unsaturated zone $1\left(\mathrm{~cm}^{* *} 3 / \mathrm{g}\right)$

R016 3 Saturated zone $\left(\mathrm{cm}^{* *} 3 / \mathrm{g}\right)$

$\mathrm{R} 016$ Leach rate (/yr)

R016 3 Solubility constant

R016 3 Distribution coefficients for daughter Th-230

R016 3 Contaminated zone $\left(\mathrm{cm}^{* *} 3 / \mathrm{g}\right)$

R016 3 Unsaturated zone $1\left(\mathrm{~cm}^{* *} 3 / \mathrm{g}\right)$

R016 3 Saturated zone $\left(\mathrm{cm}^{* *} 3 / \mathrm{g}\right)$

R016 3 Leach rate (/yr)

R016 3 Solubility constant

R016 3 Distribution coefficients for daughter U-233

R016 3 Contaminated zone $\left(\mathrm{cm}^{* *} 3 / \mathrm{g}\right)$

R016 3 Unsaturated zone $1\left(\mathrm{~cm}^{* *} 3 / \mathrm{g}\right)$

R016 3 Saturated zone $\left(\mathrm{cm}^{* *} 3 / \mathrm{g}\right)$

R016 3 Leach rate (/yr)

R016 3 Solubility constant

R016 3 Distribution coefficients for daughter U-234

R016 3 Contaminated zone $\left(\mathrm{cm}^{* *} 3 / \mathrm{g}\right)$

R016 3 Unsaturated zone $1\left(\mathrm{~cm}^{* *} 3 / \mathrm{g}\right)$

R016 3 Saturated zone $\left(\mathrm{cm}^{* *} 3 / \mathrm{g}\right)$

R016 3 Leach rate (/yr)

R016 3 Solubility constant

R016 3 Distribution coefficients for daughter U-235

R016 3 Contaminated zone $\left(\mathrm{cm}^{* *} 3 / \mathrm{g}\right)$

R016 3 Unsaturated zone $1\left(\mathrm{~cm}^{* *} 3 / \mathrm{g}\right)$

R016 3 Saturated zone $\left(\mathrm{cm}^{* *} 3 / \mathrm{g}\right)$

R016 3 Leach rate (/yr)

R016 3 Solubility constant

R017 3 Inhalation rate $\left(m^{* *} 3 / \mathrm{yr}\right)$

R017 3 Mass loading for inhalation $(\mathrm{g} / \mathrm{m} * * 3)$

R017 3 Exposure duration

R017 3 Shielding factor, inhalation

R017 3 Shielding factor, external gamma

R017 3 Fraction of time spent indoors

R017 3 Fraction of time spent outdoors (on site)

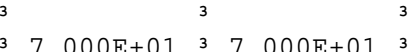

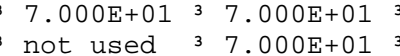

not used $37.000 \mathrm{E}+01$

$0.000 \mathrm{E}+003 \quad 0.000 \mathrm{E}+00$

$0.000 \mathrm{E}+\odot \Theta^{3} \quad 0.000 \mathrm{E}+0 \odot^{3}$

3

3. $6.00 \odot \mathrm{E}+\odot 4 \quad 3 \quad 6.000 \mathrm{E}+\odot 4$

not used 3 6.000E+०4

not used $36.000 \mathrm{E}+04$

$\begin{array}{lll}0.000 \mathrm{E}+00 & 3 & 0.000 \mathrm{E}+00^{3}\end{array}$

$\odot .000 \mathrm{E}+0 \odot^{3} \quad 0.000 \mathrm{E}+0 \odot^{3}$$$
3
$$

6. $\odot \odot \odot E+\odot 4 \quad 3 \quad 6, \odot \odot \odot E+\odot 4$ not used 3 6. $60 \odot \mathrm{E}+\odot 4$ not used $36.000 \mathrm{E}+04$

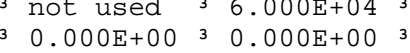

$\odot .000 \mathrm{E}+\odot \odot 3 \quad \odot .00 \odot \mathrm{E}+\odot \odot^{3}$

5. $00 \odot \mathrm{E}+01 \quad 3 \quad 5.000 \mathrm{E}+01$ not used 3 5. $5000 \mathrm{E}+01$

not used $35.000+01$

not used

$0.000 \mathrm{E}+00$ 3 $0.000 \mathrm{E}+0 \odot$

$\odot . \odot \odot \odot E+\odot \odot \quad 3 \quad 0.0 \odot \odot E+\odot \odot$

3. $5.000 \mathrm{E}+01 \quad 3 \quad 5.000 \mathrm{E}+01$

not used 3 5. $000 \mathrm{E}+01$

not used $35.000 E+01$

$0.0 \odot \odot \mathrm{E}+\odot \odot \quad 3 \quad 0.000 \mathrm{E}+\odot \odot 3$

$\odot .00 \odot \mathrm{E}+0 \odot$ з $\odot .00 \odot \mathrm{E}+0 \odot 3$

5. $000 \mathrm{E}+01 \quad 3 \quad 5.000 \mathrm{E}+01$

3 not used $35.000 \mathrm{E}+013$

not used 3 5.000E+01

$0.000 \mathrm{E}+00{ }^{3} \quad 0.000 \mathrm{E}+00^{3}$

$\odot ., 000 \mathrm{E}+0 \odot \quad 3 \quad 0,000 \mathrm{E}+0 \Theta^{3}$

$1.230 \mathrm{E}+04 \quad 3 \quad 8.400 \mathrm{E}+03$

$6.000 \mathrm{E}-04$ 3 $1.000 \mathrm{E}-04$

2.500E+01 3 3.000E+01

1. $.000 \mathrm{E}+00$ 3 $4.000 \mathrm{E}-01$

1.000E+00 3 7.000E-01

$0.000 \mathrm{E}+00{ }^{3} \quad 5.000 \mathrm{E}-01$

3 3.800E-02 3 2.500E-01

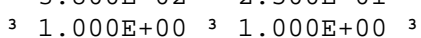

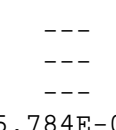

$5.784 \mathrm{E}-03$

not used

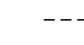

$-$

$6.767 \mathrm{E}-06$

not used

$--$

$-$

$6.767 \mathrm{E}-06$

not used

\section{- - -}

$-$

8. $089 \mathrm{E}-03$

not used

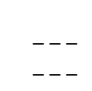

$--$

8. $089 \mathrm{E}-03$

not used

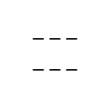

8. $089 \mathrm{E}-03$

not used

-.

$--$

$--$

$--$

$-$
$3 \operatorname{DCNUCC}(11)$

$3 \operatorname{DCNUCU}(11,1)$

3 DCNUCS(11)

3 ALEACH(11)

SOLUBK(11)

DCNUCC (14)

$\operatorname{DCNUCU}(14,1)$

DCNUCS (14)

$\operatorname{ALEACH}(14)$
$\operatorname{SOLUBK}(14)$

DCNUCC (15)

$3 \operatorname{DCNUCU}(15,1)$

DCNUCS (15)

$\operatorname{ALEACH}(15)$

SOLUBK (15)

$\operatorname{DCNUCC}(16)$

$\operatorname{DCNUCU}(16,1)$

DCNUCS (16)

ALEACH (16)

SOLUBK (16)

DCNUCC (17)

$\operatorname{DCNUCU}(17,1)$

DCNUCS (17)

ALEACH (17)

SOLUBK (17)

DCNUCC (18)

$3 \operatorname{DCNUCU}(18,1)$

DCNUCS (18)

ALEACH (18)

SOLUBK(18)

3 INHALR

3 MLINH

3 ED

3 SHF3

3 SHF1

3 FIND

3 FOTD

$>0$ shows circular AREA. $3 \mathrm{FS}$ 
Site-Specific Parameter Summary (continued)

Menu 3

Parameter

3 User

3 Default 3 (If different from user input) 3 Name

Used by RESRAD

3 Parameter

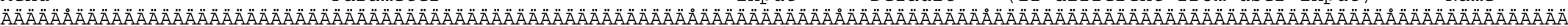

R017 3 Radii of shape factor array (used if FS = -1):

R017 3 Outer annular radius $(\mathrm{m})$ ring

(

R017 3 Outer annular radius $(\mathrm{m})$, ring 3

R017 3 Outer annular radius $(\mathrm{m})$, ring 4 :

R017 3 Outer annular radius (m), ring 5:

not used $35.000 \mathrm{E}+01$

not used $3 \quad 7.071 \mathrm{E}+01$

not used $30.000 \mathrm{E}+00$

not used $30.000 \mathrm{E}+0 \Theta^{3}$

R017 3 Outer annular radius (m), ring 6 :

3 not used 3 ०. $000 \mathrm{E}+0 \Theta^{3}$

R017 3 Outer annular radius (m), ring 6 :

$\mathrm{R} 017^{3}$ Outer annular radius $(\mathrm{m})$, ring 8:

R017 3 Outer annular radius $(\mathrm{m})$, ring 9

not used $3 \quad 0.000 \mathrm{E}+0 \Theta^{3}$

not used $30.000 \mathrm{E}+00^{3}$

3 not used $30.000 \mathrm{E}+0 \odot^{3}$

Outer annular radius (m), ring 10:

$\mathrm{R} 017$ Outer annular radius $(\mathrm{m})$, ring 11 :

R017 3 Outer annular radius $(\mathrm{m})$, ring 12 :

R017 3 Fractions of annular areas within AREA:

$0.000 \mathrm{E}+\odot \odot$

not used 3 O. $000 \mathrm{E}+00$

R017 3 Ring 1

R017 3 Ring 2

R017 3 Ring 3

R017 3 Ring 4

R017 3 Ring 5

$\begin{array}{llll}\mathrm{R} 017 & 3 & \text { Ring } & 6 \\ \mathrm{R} 017 & 3 & \text { Ring } & 7\end{array}$

R017 3 Ring 8

R017 3 Ring 9

R017 3 Ring 10

R017 3 Ring 11

R017 3 Ring 12

R018 3 Fruits, vegetables and grain consumption (kg/yr) R018 3 Leafy vegetable consumption ( $\mathrm{kg} / \mathrm{yr}$ )

R018 3 Milk consumption ( $\mathrm{L} / \mathrm{yr}$ )

R018 3 Meat and poultry consumption (kg/yr)

R018 3 Fish consumption ( $\mathrm{kg} / \mathrm{yr}$ )

R018 3 Other seafood consumption ( $\mathrm{kg} / \mathrm{yr}$ )

R018 3 Soil ingestion rate ( $g / y r)$

R018 3 Drinking water intake (L/yr)

R018 3 Contamination fraction of drinking water

R018 3 Contamination fraction of household water

R018 3 Contamination fraction of livestock water

R018 3 Contamination fraction of irrigation water

R018 3 Contamination fraction of aquatic food

R018 3 Contamination fraction of plant food

R018 3 Contamination fraction of meat

R018 3 Contamination fraction of milk$$
3
$$

R019 3 Livestock fodder intake for meat ( $\mathrm{kg} /$ day) R019 3 Livestock fodder intake for milk ( $\mathrm{kg} /$ day)

R019 3 Livestock water intake for meat ( $\mathrm{L} /$ day)

R019 3 Livestock water intake for milk (L/day)

R019 3 Livestock soil intake ( $\mathrm{kg} /$ day)

R019 3 Mass loading for foliar deposition $(\mathrm{g} / \mathrm{m} * * 3)$

not used 3 2.732E-01

not used $3 \quad 0.000 \mathrm{E}+00^{3}$

not used $30.000 \mathrm{E}+0 \odot$

not used 3 ०. $\odot 0 \odot E+\odot \odot$

not used $30.000 \mathrm{E}+0 \odot$

not used $30.000 \mathrm{E}+00^{3}$

3 not used $30.000 \mathrm{E}+0 \theta^{\circ}$

3 not used 3 Q $000 \mathrm{E}+0 \theta^{3}$

not used $3 \quad 0.000 \mathrm{E}+0 \Theta^{3}$

not used $3 \quad 0.000 \mathrm{E}+00^{3}$

not used

$0.000 \mathrm{E}+00$

not used

$1.600 \mathrm{E}+02$

not used $3 \quad 1.400 E+01$

not used 3 9.200E+O1

not used 3 6.300E+01

not used 3 5.400E+00

not used 3 9.000E-01

$1.752 \mathrm{E}+02 \quad 3 \quad 3.650 \mathrm{E}+01$

not used $35.100 \mathrm{E}+02$

not used $31.000 \mathrm{E}+0 \odot$

not used 3 1.000E $+0 \odot$

not used 31 . $000 \mathrm{E}+0 \theta^{3}$

not used $31.000 \mathrm{E}+00^{3}$

not used 3 5.000E- 01

not used

not used

not used $3-1$

$3-1$

not used

3 not used

$3^{3}$

not used $35.000 \mathrm{E}+01$

not used 3 1.600E+02

3 not used 3 (. $3.000 \mathrm{E}-01 \mathrm{C}^{3}$ $-\cdot$

$-$

$-$

$-$

$-$

$-$

$-$

$-$

$--$

$-\cdot$

$-$

$-$

$-$

$-$

$-$

$-$

$---$

$-$

$-$

$-$

$--$

- .

$--$

- - -

$-$

- -

- -

$-\cdot$

$--$

(1)

$-$

$-\ldots$

$--$

$---$
RAD_SHAPE ( 1$)$

RAD_SHAPE ( 2$)$

3 RAD_SHAPE ( 3 )

3 RAD_SHAPE ( 4$)$

3 RAD_SHAPE ( 5 )

3 RAD_SHAPE ( 6)

3 RAD_SHAPE $(7)$

3 RAD_SHAPE ( 8)

3 RAD_SHAPE ( 9)

RAD_SHAPE $(10)$

3 RAD_SHAPE (11)

3

$3 \operatorname{FRACA}(1)$

3 FRACA ( 2)

FRACA ( 3)

FRACA( 4$)$

FRACA( 5)

FRACA ( 6)

3 FRACA ( 7)

FRACA ( 8)

FRACA( 9)

3 FRACA (10)

$\begin{array}{ll}3 & \operatorname{FRACA}(11) \\ 3 & \operatorname{FRACA}(12)\end{array}$

3 DIET(1)

$3 \operatorname{DIET}(3)$

$3 \operatorname{DIET}(4)$

$3 \operatorname{DIET}(5)$

$3 \operatorname{DIET}(6)$

3 SOIL

3 DWI

3 FDW

3 FHHW

3 FLW

3 FIRW

3 FPLANT

3 FMEAT

3 FMILK

3 LFI5

3 LFI6

3 LWI5

3 LWI6

3 MLFD 
Site-Specific Parameter Summary (continued)

$\odot^{\circ}$ Menu 3

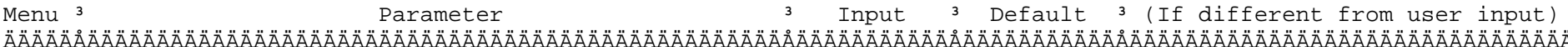

R019 3 Depth of soil mixing layer $(\mathrm{m})$

R019 3 Depth of roots $(\mathrm{m})$

R019 3 Drinking water fraction from ground water

R019 3 Household water fraction from ground water

R019 3 Livestock water fraction from ground water

R019 3 Irrigation fraction from ground water

R19B 3 Wet weight crop yield for Non-Leafy $\left(\mathrm{kg} / \mathrm{m}^{* *} 2\right)$

R19B 3 Wet weight crop yield for Leafy $(\mathrm{kg} / \mathrm{m} * * 2)$

R19B 3 Wet weight crop yield for Fodder $\left(\mathrm{kg} / \mathrm{m}^{* *} 2\right)$

R19B 3 Growing Season for Non-Leafy (years)

R19B 3 Growing Season for Leafy (years)

R19B 3 Growing Season for Fodder (years)

R19B 3 Translocation Factor for Non-Leafy

R19B 3 Translocation Factor for Leafy

R19B 3 Translocation Factor for Fodder 3 not used $31.000 \mathrm{E}+00^{3}$

R19B 3 Dry Foliar Interception Fraction for Non-Leafy 3 not used $32.500 \mathrm{E}-01$

R19B 3 Dry Foliar Interception Fraction for Leafy 3 not used $32.500 \mathrm{E}-01$

R19B 3 Dry Foliar Interception Fraction for Fodder 3 not used $32.500 \mathrm{E}-01^{3}$

R19B 3 wet Foliar Interception Fraction for Non-Leafy 3 not used $32.500 \mathrm{E}-01$

R19B 3 Wet Foliar Interception Fraction for Leafy 3 not used 3 2.500E-01

R19B 3 Wet Foliar Interception Fraction for Fodder

R19B 3 Weathering Removal Constant for Vegetation

C14 3 C-12 concentration in water $\left(\mathrm{g} / \mathrm{cm}^{* *} 3\right)$

C14 3 C-12 concentration in contaminated soil $(\mathrm{g} / \mathrm{g})$

C14 3 Fraction of vegetation carbon from soil

C14 3 Fraction of vegetation carbon from air

C14 3 C-14 evasion layer thickness in soil (m)

C14 3 C-14 evasion flux rate from soil ( $1 / \mathrm{sec})$

C14 3 C-12 evasion flux rate from soil (1/sec)

C14 3 Fraction of grain in beef cattle feed

C14 3 Fraction of grain in milk cow feed

C14 3 DCF correction factor for gaseous forms of $\mathrm{C} 14$

STOR 3 Storage times of contaminated foodstuffs (days):

STOR 3 Fruits, non-leafy vegetables, and grain

STOR ${ }^{3}$ Leafy vegetables

STOR 3 Milk

STOR ${ }^{3}$ Meat and poultry

STOR 3 Fish

STOR 3 Crustacea and mollusks

STOR 3 Well water

STOR 3 Surface water

STOR ${ }^{3}$ Livestock fodder

R021 3 Thickness of building foundation (m)

R021 3 Bulk density of building foundation $\left(\mathrm{g} / \mathrm{cm}^{* *} 3\right)$

R021 3 Total porosity of the cover material

R021 3 Total porosity of the building foundation

R०21 3 Volumetric water content of the cover material

3 User

3 Default

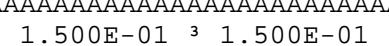

not used 3 a $000 \mathrm{E}-01$

not used $31.000 \mathrm{E}+\odot \odot$

not used $31.00 \odot \mathrm{E}+\odot \odot$

not used $31.000 \mathrm{E}+0 \odot$

not used

$1.000 \mathrm{E}+00^{\circ}$

not used

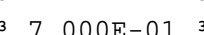

not used

not used $31.500 \mathrm{E}+00$

not used $31.100 \mathrm{E}+00$

not used $31.700 \mathrm{E}-01$

not used 3 2.500E-01

not used 3 8.000E-02

not used 3 1.000E-01

3 not used 3 1.000E+00

not used

2. $500 \mathrm{E}-01$

not used

not used $32.000 \mathrm{E}^{3}-5_{3}$

not used 3 3.000E-02

not used $32.000 \mathrm{E}-02$

not used 3 9.800E-01

not used 3 3.000E-01

not used 3 7.000E-07

not used $31.000 \mathrm{E}-10$

not used 3 8 .000E-01

not used 3 2.000E-01

Used by RESRAD 3 Paramete

Name
DäÄÄÄÄÄÄÄÄÄÄÄ

3 1.400E+01 3 1.400E+01

$1.000 \mathrm{E}+003 \quad 1.000 \mathrm{E}+00$

$1.000 \mathrm{E}+00$ 3 $1.000 \mathrm{E}+00$

2. $000 \mathrm{E}+01$ 3 3 2. $000 \mathrm{E}+01$

$7.000 \mathrm{O}+00^{3} 7.000 \mathrm{C}+0 \mathrm{O}_{3}$

$37.000 \mathrm{E}+003 \quad 7.000 \mathrm{E}+00$

1. $00 \odot \mathrm{E}+0 \odot 3 \quad 1.000 \mathrm{E}+0 \odot$

$1.000 \mathrm{E}+00^{3} 1.000 \mathrm{E}+0 \Theta^{3}$

$4.500 E+013 \quad 4.500 E+01$

3 not used $31.500 \mathrm{E}-01$

3 not used 3 2.400E +00

3 not used 34 . $4000-01$

3 not used 3 1. $0 \odot \odot \mathrm{E}-01$

3 not used 3 5.00०E-02

\begin{tabular}{|c|c|c|}
\hline--- & 3 & \\
\hline - . - & 3 & DROOT \\
\hline - - - & 3 & FGWDW \\
\hline-- & 3 & FGWHH \\
\hline - - - & 3 & FGWLW \\
\hline - - - & $\begin{array}{l}3 \\
3\end{array}$ & FGWIR \\
\hline - - - & 3 & YV(1) \\
\hline- & 3 & YV (2) \\
\hline$-\ldots$ & 3 & $Y V(3)$ \\
\hline - - - & 3 & $\operatorname{TE}(1)$ \\
\hline - - - & 3 & $\operatorname{TE}(2)$ \\
\hline-- & 3 & TE (3) \\
\hline-- & 3 & $\operatorname{TIV}(1)$ \\
\hline - - - & 3 & $\operatorname{TIV}(2)$ \\
\hline - - - & 3 & $\operatorname{TIV}(3)$ \\
\hline - - - & 3 & $\operatorname{RDRY}(1)$ \\
\hline-- & 3 & $\operatorname{RDRY}(2)$ \\
\hline$-\ldots$ & 3 & $\operatorname{RDRY}(3)$ \\
\hline-- & 3 & RWET (1) \\
\hline 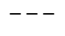 & 3 & RWET (2) \\
\hline--- & 3 & RWET (3) \\
\hline-- & $\begin{array}{l}3 \\
3\end{array}$ & WLAM \\
\hline-- & 3 & C12WTR \\
\hline-- & 3 & C12CZ \\
\hline-- & 3 & CSOIL \\
\hline - - - & 3 & CAIR \\
\hline- & 3 & DMC \\
\hline- & 3 & EVSN \\
\hline- & 3 & REVSN \\
\hline - - - & 3 & AVFG4 \\
\hline- & 3 & AVFG5 \\
\hline-- & 3 & $\mathrm{CO} 2 \mathrm{~F}$ \\
\hline & $\begin{array}{l}3 \\
3\end{array}$ & \\
\hline - - - & 3 & STOR_T(1) \\
\hline - _ - & 3 & STOR_T (2) \\
\hline - & 3 & STOR_T (3) \\
\hline- & 3 & STOR_T (4) \\
\hline- & 3 & STOR_T (5) \\
\hline- & 3 & STOR_T (6) \\
\hline - & 3 & STOR_T (7) \\
\hline- & 3 & STOR_T ( 8 ) \\
\hline- & 3 & STOR_T(9) \\
\hline & 3 & \\
\hline & 3 & FLOOR1 \\
\hline & 3 & DENSFL \\
\hline 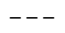 & 3 & TPCV \\
\hline & 3 & TPFL \\
\hline & 3 & $\mathrm{PH} 20 \mathrm{CV}$ \\
\hline
\end{tabular}


Site-Specific Parameter Summary (continued)

Menu 3

3 Default

$3 \quad$ Used by RESRAD

Used by RESRAD 3 Parameter

Menu 3 Parameter 3 Input 3 Default 3 (If different from user input) 3 Name

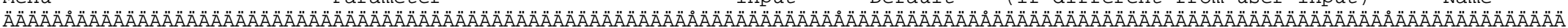

RO21 3 Volumetric water content of the foundation

R021 3 Diffusion coefficient for radon gas $(\mathrm{m} / \mathrm{sec})$ :

$\mathrm{R} 021^{3}$ in cover material

RO21 3 in foundation material

RO21 3 in contaminated zone soil

R021 3 Radon vertical dimension of mixing $(\mathrm{m})$

3 not used $3.000 \mathrm{E}-02$

3

3. $000 \mathrm{E}-02$

not used

2. $000 \mathrm{E}-066^{3}$

not used $32.000 \mathrm{E}-07$

R०21 3 Average building air exchange rate $(1 / \mathrm{hr})$

R021 3 Height of the building (room) (m)

RO21 3 Building interior area factor

R021 3 Building depth below ground surface $(\mathrm{m})$

Ro21 3 Emanating power of $\mathrm{Rn}-222$ gas

not used 3 2. $\odot \odot \odot E+\odot \odot$

not used $35.000 \mathrm{E}-01$

not used $32.500 E+00$

not used 3 .

R021 3 Emanating power of $\mathrm{Rn}-220$ gas

not used

not used $3-1.000 \mathrm{E}+00^{3}$

not used $32.500 \mathrm{E}-01$

not used 3 1.500E- 01

TITL 3 Number of graphical time points

TITL 3 Maximum number of integration points for dose

$\begin{array}{llll}32 & 3 & \ldots & 3\end{array}$

TIT Maximu noints for dose

$25732-\cdots$

..

$--$

$-$

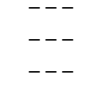

- -

$---$

$--$

$--\cdot$

- . -

$-$
3 PH2OFL

3 DIFCV

3 DIFFL

3 DIFCZ

3 HMIX

3 REXG

3 HRM

3 FAI

$\begin{array}{ll}3 & \operatorname{EMANA}(1) \\ 3 & \operatorname{EMANA}(2)\end{array}$

3 NPTS

3 LYMAX

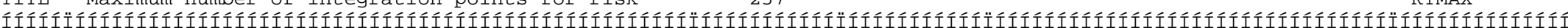

Summary of Pathway Selections

Pathway 3 User Selection

ÄÄÄÄÄÄÄÄÄÄÄÄÄÄÄÄÄÄÄÄÄÄÄÄÄÄÄÄÄÄÄÄÄÄÄÄÄÄÄÄÄÄÄÄÄÄÄÄÄÄÄÄ

1 - - external gamma 3 active

2 - - inhalation (w/o radon) ${ }^{3}$ active

3 -- plant ingestion 3 suppressed

4 -- meat ingestion 3 suppressed

5 - - milk ingestion $\quad 3 \quad$ suppressed

6 - - aquatic foods 3 suppressed

7 - - drinking water 3 suppressed

8 -- soil ingestion 3 active

9 - - radon 3 suppressed

Find peak pathway doses 3 suppressed

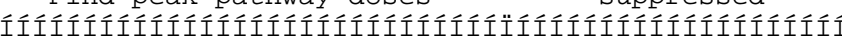


1RESRAD, Version $6.3 \quad$ T « Limit $=180$ days Summary : CAU 476

Contaminated Zone Dimensions ÄÄÄÄÄÄÄÄÄÄÄÄÄÄÄÄÄÄÄÄÄÄÄÄÄÄÄÄÄÄÄ

Area: $\quad 100.00$ square meters

Thickness: $\quad 0.15$ meters

Cover Depth: $\quad 0.00$ meters

Initial soil concentrations, pCi/g ÄÄÄÄÄÄÄÄÄÄÄÄÄÄÄÄÄÄÄÄÄÄÄÄÄÄÄÄÄÄÄÄÄÄÄ
Am-241
$2.800 \mathrm{E}+00$
Co- 60
$1.760 \mathrm{E}+\odot \odot$
Cs -137
$3.820 \mathrm{E}+02$
$\mathrm{Pu}-238$
$1.030 \mathrm{E}+00$
$\mathrm{Pu}-239$
$9.600 \mathrm{E}+00$
$\mathrm{Sb}-125$
$3.000 \mathrm{E}+\odot \odot$
Sr -90
1. $300 \mathrm{E}+01$

Total Dose TDOSE(t), mrem/yr

Basic Radiation Dose Limit $=2.500 \mathrm{E}+01 \mathrm{mrem} / \mathrm{yr}$

Total Mixture Sum $M(t)=$ Fraction of Basic Dose Limit Received at Time $(t)$

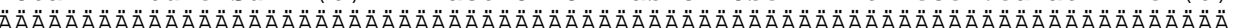

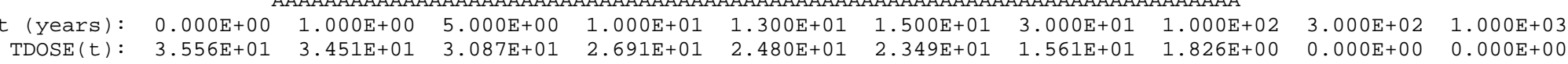

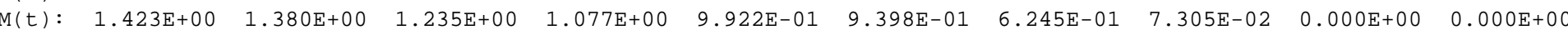

OMaximum TDOSE(t): $3.556 \mathrm{E}+01 \mathrm{mrem} / \mathrm{yr}$ at $\mathrm{t}=0.000 \mathrm{E}+00$ years 
Total Dose Contributions $\operatorname{TDOSE}(i, p, t)$ for Individual Radionuclides (i) and Pathways $(p)$

As mrem/yr and Fraction of Total Dose At $t=0.000 \mathrm{E}+00$ years

$$
\text { Water Independent Pathways (Inhalation excludes radon) }
$$

$$
\text { Ground Inhalation } 1 \text { Radon } 4 \text { Plant } \quad \text { Meat }
$$

Radio - ÄÄÄÄÄÄÄÄÄÄÄÄÄÄÄÄA ӒÄÄÄÄÄÄÄÄÄÄÄÄÄÄÄ ÄÄÄÄÄÄÄÄÄÄÄÄÄÄÄÄÄ ÄÄÄÄÄÄÄÄÄÄÄÄÄÄÄÄ M̈̈̈̈̈̈̈̈̈A 
Total Dose Contributions TDOSE( $i, p, t)$ for Individual Radionuclides ( $i$ ) and Pathways ( $p$ )

As $\mathrm{mrem} / \mathrm{yr}$ and Fraction of Total Dose At $t=1.000 \mathrm{E}+00$ years

$$
\text { Water Independent Pathways (Inhalation excludes radon) }
$$

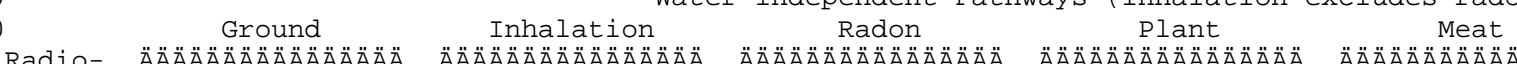

Radio - ÄÄÄÄÄÄÄÄÄÄÄÄÄÄÄA Ӓ̈̈̈ÄÄÄÄÄÄÄÄÄÄÄÄÄ ÄÄÄÄÄÄÄÄÄÄÄÄÄÄÄÄ Ä ÄÄÄÄÄÄÄÄÄÄÄÄÄÄÄ

\begin{tabular}{|c|c|c|c|c|c|c|c|c|c|c|c|c|c|c|}
\hline & & & & & & & & & & & & & & \\
\hline $\begin{array}{l}\text { Nuclide } \\
\text { ÄÄÄÄÄÄÄÄ }\end{array}$ & 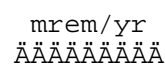 & $\begin{array}{l}\text { fract. } \\
\ddot{A} A \ddot{A} A \ddot{A} A \ddot{A}\end{array}$ & $\begin{array}{l}m r e m / y r \\
\ddot{A} \overline{A ̈ A ̈ A ̈ A ̈ A ̈ A ̈ A ̈ A ~}\end{array}$ & $\begin{array}{l}\text { fract } \\
\text { ÄÄÄÄÄÄ }\end{array}$ & 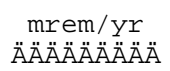 & $\begin{array}{l}\text { fract. } \\
\ddot{A} \ddot{A} \ddot{A} \ddot{A} A ̈ A ̈\end{array}$ & $\begin{array}{c}m r e m / y r \\
\ddot{A} \ddot{A} \ddot{A} \ddot{A} \ddot{A} \ddot{A} \ddot{A} \ddot{A}\end{array}$ & $\begin{array}{l}\text { fract } \\
\ddot{A} A \ddot{A} A \ddot{A} A \ddot{A}\end{array}$ & $\begin{array}{r}m r e m / y r \\
\ddot{A} \ddot{A} \ddot{A} \ddot{A} \ddot{A} \ddot{A}\end{array}$ & $\begin{array}{l}\text { fract. } \\
\ddot{A} A ̈ A ̈ A ̈ A ̈ A ̈\end{array}$ & 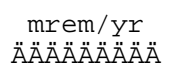 & $\begin{array}{l}\text { fract. } \\
\ddot{A} \ddot{A} \ddot{A} A \ddot{A} A ̈\end{array}$ & ÄÄÄÄÄÄÄÄ & $\begin{array}{l}\text { fract } \\
\ddot{A ̈ A ̈ A ̈ A ̈ A ̈ A ̈ ~}\end{array}$ \\
\hline & & & & & & & & & & & & & & \\
\hline & & & & & & & & & & & & & & \\
\hline & & & & & & & & & & & & & & \\
\hline & & & & & & & & & & & & & & \\
\hline & & & & & & & & & & & & & & \\
\hline$b-125$ & 6.90 & 0.0002 & $E-\odot 8$ & & $+\odot \odot$ & & $+\infty \odot$ & & $E+\odot \odot$ & & $+0 \odot$ & & -07 & \\
\hline & & & & & & & & & & & & & & \\
\hline & & & & & & & & & & & & & & \\
\hline & 3. & & 02 & & $\odot .000 \mathrm{E}$ & & $1.000 t+00$ & & ๑. $000 \mathrm{E}+\odot \odot$ & & $.000 \mathrm{\theta}+0 \odot$ & & $46 \mathrm{E}-0$ & \\
\hline
\end{tabular}

М̈̈̈̈̈̈̈ÄÄÄÄÄÄA

Soil

Total Dose Contributions $\operatorname{TDOSE}(i, p, t)$ for Individual Radionuclides (i) and Pathways $(p)$ As $\mathrm{mrem} / \mathrm{yr}$ and Fraction of Total Dose At $t=1.000 \mathrm{E}+00$ years As mrem/yr and Fraction of Total Dose At
Water Dependent Pathways

Water

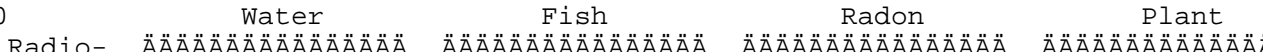
nucli

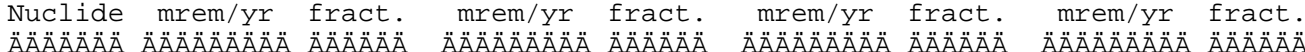

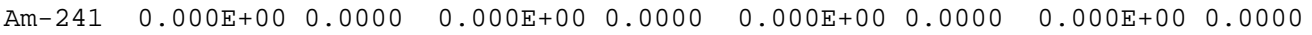

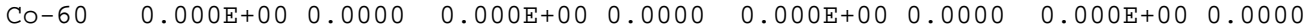

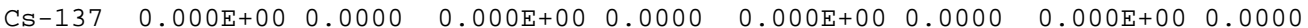
$\mathrm{Pu}-238 \quad 0.000 \mathrm{E}+00$ 0.0000 $0.000 \mathrm{E}+000.0000-0.000 \mathrm{E}+000.0000 \quad 0.000 \mathrm{E}+000.0000$ Pu-238 $0.000 E+00$.

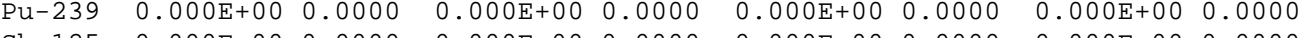

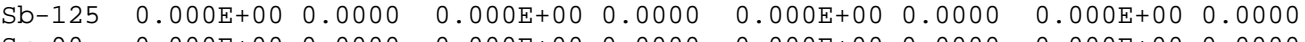

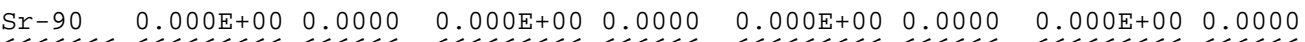

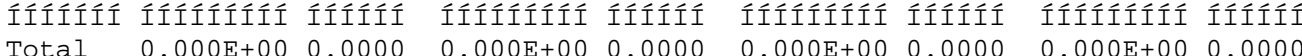

Meat АÄÄÄÄÄÄÄÄÄÄÄÄÄÄ̈̈ $\mathrm{mrem} / \mathrm{yr}$ fract ÄÄÄÄÄÄÄÄÄ ÄÄÄÄÄÄ $0.000 \mathrm{E}+00 \quad 0.0000$ $0.000 \mathrm{E}+00$ 0. 0000 0.000 $0.000 \mathrm{E}+0 \odot \quad 0.0000$ $\odot .000 \mathrm{E}+0 \odot \quad 0.000 \odot$ $0.000 \mathrm{E}+00 \quad 0.0000$ Íííííííi ííííí $0.000 \mathrm{E}+0 \odot$ ०.००००
Milk ÄÄÄÄÄล̈ÄÄÄÄÄÄÄÄÄ mrem/yr fract. ÄÄÄÄÄÄÄÄ ÄÄÄÄÄÄ $\odot .000 \mathrm{E}+00 \quad 0.0000$ $0.000 \mathrm{E}+00 \quad 0.0000$ $\odot .000 \mathrm{E}+0 \odot \quad \odot .000 \odot$ $0.000 \mathrm{E}+00 \quad 0.0000$ $0.000 \mathrm{E}+00 \quad 0.0000$ $\odot .000 \mathrm{E}+0 \odot \quad 0.0000$ $\odot .000 \mathrm{E}+0 \odot \quad \odot .000 \odot$ íííííííi ííííí $\odot .000 \mathrm{E}+000.0000$
All Pathways* Ӓ̈ÄÄÄÄÄÄÄÄÄÄÄÄÄÄ mrem/yr fract. ÄÄÄÄÄÄÄÄ ÄÄÄÄÄÄ 2.380E-02 0.0007 $\begin{array}{lll}5.878 \mathrm{E}-01 & 0.0170\end{array}$ $3.380 \mathrm{E}+01 \quad 0.9796$ $6.643 \mathrm{E}-03 \quad 0.0002$ $6.884 \mathrm{E}-02 \quad 0.0020$ $6.900 \mathrm{E}-03 \quad 0.0002$ $9.988 \mathrm{E}-03 \quad 0.0003$ IIIííííí ííííí $3.451 \mathrm{E}+011.0000$

$\odot *$ Sum of all water independent and dependent pathways. 
Total Dose Contributions $\operatorname{TDOSE}(i, p, t)$ for Individual Radionuclides (i) and Pathways $(p)$

As $\mathrm{mrem} / \mathrm{yr}$ and Fraction of Total Dose At $t=5.000 \mathrm{E}+00$ years

$$
\text { Water Independent Pathways (Inhalation excludes radon) }
$$

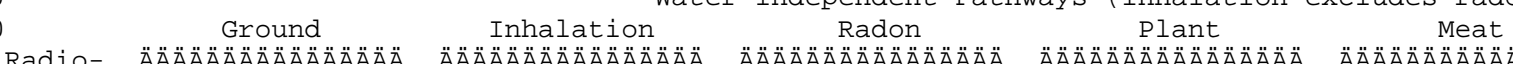

Radio- AAÄÄÄÄÄÄÄÄÄÄA ÄÄÄÄÄÄÄÄÄÄÄÄÄÄÄ ÄÄÄÄÄÄÄÄÄÄÄÄÄÄÄÄ ÄÄÄÄÄÄÄÄÄÄÄÄÄÄÄÄ

\begin{tabular}{|c|c|c|c|c|c|c|c|c|c|c|c|c|c|c|}
\hline $\begin{array}{l}\text { Nuclide } \\
\text { ÄÄÄÄÄÄÄÄ }\end{array}$ & 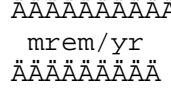 & $\begin{array}{l}\text { fract } \\
\ddot{A} A \ddot{A} A \ddot{A} A \ddot{A}\end{array}$ & 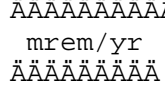 & $\begin{array}{l}\text { fract. } \\
\ddot{A} A ̈ A ̈ A ̈ A ̈ A ̈\end{array}$ & 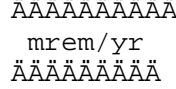 & $\begin{array}{l}\text { fract } \\
\ddot{A} A \ddot{A} A \ddot{A} A ̈ A ̈\end{array}$ & 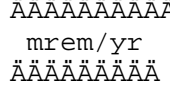 & $\begin{array}{l}\text { fract } \\
\ddot{A} A \ddot{A} \ddot{A} A \ddot{A} A\end{array}$ & $\begin{array}{c}m r e m / y r \\
A \ddot{A} A \ddot{A} A \ddot{A} \ddot{A} \ddot{A}\end{array}$ & $\begin{array}{l}\text { fract } \\
\ddot{A} A \ddot{A} A \ddot{A} A ̈ A ̈\end{array}$ & $\begin{array}{c}m r e m / y r \\
\ddot{A} A \ddot{A} A \ddot{A} A \ddot{A} A \ddot{A}\end{array}$ & $\begin{array}{l}\text { fract } \\
\ddot{A} A \ddot{A} A \ddot{A} A \ddot{A}\end{array}$ & 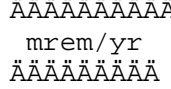 & $\begin{array}{l}\text { fract } \\
\ddot{A} A \ddot{A} A \ddot{A} A ̈ A\end{array}$ \\
\hline Am-241 & $3.434 \mathrm{E}-03$ & $\odot .0001$ & $1.209 \mathrm{E}-02$ & 0.0004 & $\odot .000 \mathrm{E}+0 \odot$ & 0.0000 & $\odot .000 \mathrm{E}+\odot \odot$ & 0.0000 & $\odot .000 \mathrm{E}+0 \odot$ & 0.0000 & $\odot .000 \mathrm{E}+\odot \odot$ & 0.0000 & $5.801 \mathrm{E}-03$ & $\odot .00 \odot 2$ \\
\hline Co-60 & $3.416 \mathrm{E}-01$ & 0.0111 & $2.046 E-\odot 6$ & $\odot .0 \odot \odot \odot$ & $\odot . \odot \odot \odot E+\odot \odot$ & $\odot .0 \odot \odot \odot$ & $\odot . \odot \odot \odot E+\odot \odot$ & $\odot . \odot \odot \odot \odot$ & $\odot . \odot \odot \odot E+\odot \odot$ & $\odot .0 \odot \odot \odot$ & $\odot . \odot \odot \odot E+\odot \odot$ & & & \\
\hline Cs - 137 & $3 . \odot 41 E+01$ & $\odot .9852$ & $1.175 \mathrm{E}-\odot 4$ & $\odot .0 \odot \odot \odot$ & $\odot . \odot \odot \odot E+\odot \odot$ & $\odot . \odot \odot \odot \odot$ & $\odot . \odot \odot \odot E+\odot \odot$ & $\odot .000 \odot$ & $\odot . \odot \odot \odot E+\odot \odot$ & $\odot .0 \odot \odot \odot$ & $\odot . \odot \odot \odot E+\odot \odot$ & $\odot .0 \odot \odot \odot$ & $1.078 \mathrm{E}-02$ & 0.0003 \\
\hline Pu-238 & $5.121 \mathrm{E}-06$ & $\odot .00 \odot \odot$ & $4.231 \mathrm{E}-03$ & 0.0001 & $\odot . \odot \odot \odot E+\odot \odot$ & $\odot .000 \odot$ & $\odot .000 \mathrm{E}+\odot \odot$ & 0.0000 & $\odot .00 \odot E+\odot \odot$ & 0.0000 & $\odot .00 \odot E+\odot \odot$ & $\odot .0000$ & $2.022 \mathrm{E}-03$ & 0001 \\
\hline Pu-239 & $8.536 \mathrm{E}-05$ & 0.0000 & $4.507 \mathrm{E}-02$ & 0.0015 & $\odot . \odot \odot ० E+\odot \odot$ & $\odot .0000$ & $\odot . \odot \odot \odot E+\odot \odot$ & 0.0000 & $\odot . \odot \odot \odot E+\odot \odot$ & $\odot .000 \odot$ & $\odot . \odot \odot \odot E+\odot \odot$ & ๑.๑००० & $E-\odot 2$ & 000 \\
\hline $\mathrm{Sb}-125$ & $4.606 \mathrm{E}-\odot 7$ & $\odot . \odot \odot \odot \odot$ & 1. $060 \mathrm{E}-12$ & $\odot .0 \odot \odot \odot$ & $\odot . \odot \odot \odot E+\odot \odot$ & $\odot .0 \odot \odot \odot$ & $\odot . \odot \odot \odot E+\odot \odot$ & $\odot .0 \odot \odot \odot$ & $\odot . \odot \odot \odot E+\odot \odot$ & $\odot .0 \odot \odot \odot$ & $\odot . \odot \odot \odot E+\odot \odot$ & ๑.๑०९९ & $1.632 \mathrm{E}-11$ & $\odot .0000$ \\
\hline $\mathrm{Sr}-90$ & $7.305 \mathrm{E}-03$ & 0.0002 & $1.519 \mathrm{E}-04$ & 0.0000 & $\odot .000 \mathrm{E}+\odot \odot$ & 0.0000 & & & & & $\odot .00 \odot E+\odot \odot$ & & $1.038 \mathrm{E}-03$ & \\
\hline & & & & & & & & & & & & & & \\
\hline Total & 3. $076 \mathrm{E}+01$ & 0.9967 & $6.166 \mathrm{E}-02$ & 0.0020 & $\odot . \odot \odot \odot E+\odot \odot$ & .0000 & $\odot . \odot \odot \odot E+\odot \odot$ & $\odot .0 \odot \odot \odot$ & $\odot . \odot \odot \odot E+\odot \odot$ & $\odot .000 \odot$ & $\odot . \odot \odot \odot E+\odot \odot$ & $\odot .000 \odot$ & $4.143 E-02$ & $\odot .001$ \\
\hline
\end{tabular}

М̈̈̈̈̈ÄÄÄÄÄÄÄA

Soil

Total Dose Contributions TDOSE(i,p,t) for Individual Radionuclides (i) and Pathways $(p)$

0 As $\mathrm{mrem} / \mathrm{yr}$ and Fraction of Total Dose At $t=5.000 \mathrm{E}+0 \odot$ years

$$
\begin{array}{r}
\text { As } \mathrm{mrem} / \mathrm{yr} \text { and Fraction of Total Dose At } t \\
\text { Water Dependent Pathways }
\end{array}
$$

\section{Fish}

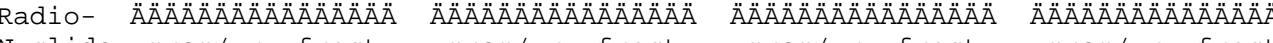

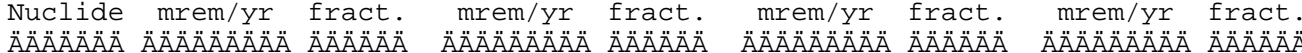

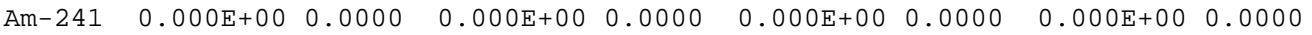

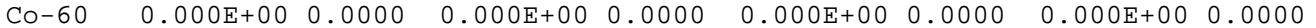

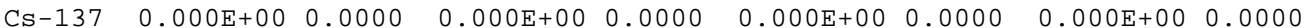
$\mathrm{Pu}-2380.000 \mathrm{E}+00$ 0.0000 $0.000 \mathrm{E}+00$ 0.0000 $0.000 \mathrm{E}+00$ 0.0000 $0.000 \mathrm{E}+000.0000$ Pu-238

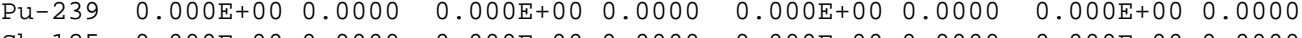

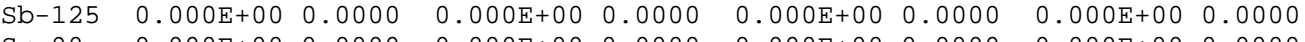

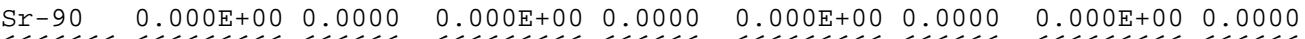

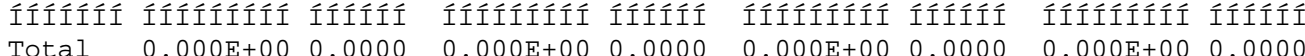

$\odot *$ Sum of all water independent and dependent pathways.

\author{
Meat
} ÄÄÄÄÄÄÄÄÄÄÄÄÄÄA mrem/yr fract ÄÄÄÄÄÄÄÄÄ ÄÄÄÄÄ̈ $0.000 \mathrm{E}+00$ 0.000९ $0.000 \mathrm{E}+000.00000$ $0.000 \mathrm{E}+000.0000$ $0.000 \mathrm{E}+000.0000$ $0.000 \mathrm{E}+000.0000$ $0.000 \mathrm{E}+000.0000$ $\odot .000 \mathrm{E}+00 \quad 0.0000$ ÍÍÍÍÍÍÍ ÍÍÍÍÍ́
Milk ÄÄÄÄÄÄÄÄÄÄÄÄÄÄÄÄ ÄÄÄÄÄÄÄÄÄÄÄÄÄÄÄÄ mrem/yr fract. mrem/yr fract. ÄÄÄÄÄÄÄÄÄ ÄÄÄÄÄÄ Ä $\begin{array}{lllll}0.000 \mathrm{E}+00 & 0.0000 & 2.132 \mathrm{E}-02 & 0.0007\end{array}$ $0.000 \mathrm{E}+00 \quad 0.0000 \quad 3.417 \mathrm{E}-01 \quad 0.0111$ $\odot .00 \odot \mathrm{E}+0 \odot \quad 0.000 \odot \quad 3.042 \mathrm{E}+01 \quad 0.9856$ $0.000 \mathrm{E}+000.0000 \quad 6.258 \mathrm{E}-03 \quad 0.0002$ $0.000 \mathrm{E}+00 \quad 0.0000 \quad 6.692 \mathrm{E}-02 \quad 0.0022$ $\odot .000 \mathrm{E}+0 \odot \quad 0.000 \odot \quad 4.607 \mathrm{E}-07 \quad 0.00 \odot \odot$

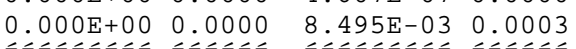
Íííííííi ííííí ííííííí ííííí $0.000 \mathrm{E}+00 \quad 0.0000 \quad 3.087 \mathrm{E}+01 \quad 1.0000$ 
Total Dose Contributions $\operatorname{TDOSE}(i, p, t)$ for Individual Radionuclides (i) and Pathways $(p)$

As mrem/yr and Fraction of Total Dose At $t=1.000 E+01$ years

$$
\text { Water Independent Pathways (Inhalation excludes radon) }
$$

$$
\begin{array}{ccc}
\text { Inhalation } & \text { Radon } & \text { Mlant } \\
& &
\end{array}
$$

Radio - ÄÄÄÄÄÄÄÄÄÄÄÄÄÄÄÄA

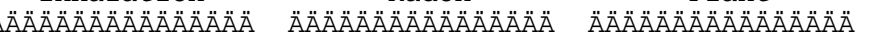
M̈̈̈̈̈̈̈̈̈A 
Total Dose Contributions TDOSE(i,p,t) for Individual Radionuclides (i) and Pathways (p)

As mrem/yr and Fraction of Total Dose At $t=1.300 E+01$ years

$$
\text { Water Independent Pathways (Inhalation excludes radon) }
$$

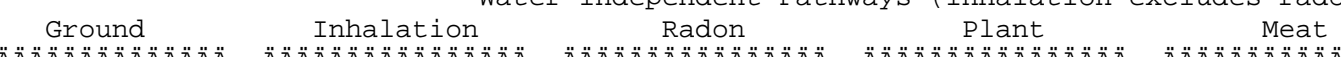

Radio - ÄÄÄÄÄÄÄÄÄÄÄÄÄÄÄÄA АÄÄÄÄÄÄÄÄÄÄÄÄÄÄÄ ÄÄÄÄÄÄÄÄÄÄÄÄÄÄÄÄ ÄÄÄÄÄÄÄÄÄÄÄÄÄÄÄÄ M̈̈̈̈̈̈̈̈̈A 
Total Dose Contributions TDOSE(i,p,t) for Individual Radionuclides (i) and Pathways (p)

As mrem/yr and Fraction of Total Dose At $t=1.500 E+01$ years

$$
\text { Water Independent Pathways (Inhalation excludes radon) }
$$

$$
\text { Ground Inhalation } 1 \text { Radon } 4 \text { Plant } \quad \text { Meat }
$$

Radio - ÄÄÄÄÄÄÄÄÄÄÄÄÄÄÄÄA АÄÄÄÄÄÄÄÄÄÄÄÄÄÄÄ ÄÄÄÄÄÄÄÄÄÄÄÄÄÄÄÄ ÄÄÄÄÄÄÄÄÄÄÄÄÄÄÄÄ M̈̈̈̈̈̈̈̈̈A 
Total Dose Contributions $\operatorname{TDOSE}(i, p, t)$ for Individual Radionuclides (i) and Pathways $(p)$

As mrem/yr and Fraction of Total Dose At $t=3.000 E+01$ years

$$
\text { Water Independent Pathways (Inhalation excludes radon) }
$$

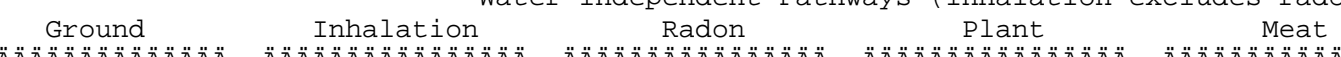

Radio - ÄÄÄÄÄÄÄÄÄÄÄÄÄÄÄÄA ӒÄÄÄÄÄÄÄÄÄÄÄÄÄÄÄ ÄÄÄÄÄÄÄÄÄÄÄÄÄÄÄÄÄ ÄÄÄÄÄÄÄÄÄÄÄÄÄÄÄÄ M̈̈̈̈̈̈̈̈̈A 
Total Dose Contributions TDOSE(i,p,t) for Individual Radionuclides (i) and Pathways (p)

As mrem/yr and Fraction of Total Dose At $t=1.000 E+02$ years

$$
\text { Water Independent Pathways (Inhalation excludes radon) }
$$

$$
\text { Ground Inhalation } 1 \text { Radon } 4 \text { Plant } \quad \text { Meat }
$$

Radio- ÄÄÄÄÄÄÄÄÄÄÄÄÄÄÄ Ӓ̈̈̈̈ӒÄÄÄÄÄÄÄÄÄÄÄ ÄÄÄÄÄÄÄÄÄÄÄÄÄÄÄÄ ÄÄÄÄÄÄÄÄÄÄÄÄÄÄÄÄ Më̈̈̈̈̈̈̈̈A 
Total Dose Contributions $\operatorname{TDOSE}(i, p, t)$ for Individual Radionuclides (i) and Pathways $(p)$

As mrem/yr and Fraction of Total Dose At $t=3.000 E+02$ years

$$
\text { Water Independent Pathways (Inhalation excludes radon) }
$$

$$
\begin{array}{ccc}
\text { Inhalation } & \text { Radon } & \text { Mlant } \\
& &
\end{array}
$$

Radio - ÄÄÄÄÄÄÄÄÄÄÄÄÄÄÄÄA

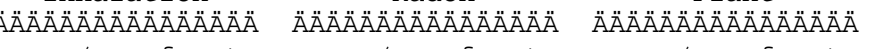
M̈̈̈̈̈̈̈̈̈A 
Total Dose Contributions $\operatorname{TDOSE}(i, p, t)$ for Individual Radionuclides (i) and Pathways $(p)$

As mrem/yr and Fraction of Total Dose At $t=1.000 E+03$ years

$$
\text { Water Independent Pathways (Inhalation excludes radon) }
$$

$$
\begin{array}{ccc}
\text { Inhalation } & \text { Radon } & \text { Meat } \\
& &
\end{array}
$$

Radio - ÄÄÄÄÄÄÄÄÄÄÄÄÄÄÄÄA

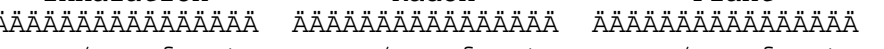
М̈̈̈̈̈̈̈̈̈̈A 
$\odot$ Parent Product Thread

(i) (j) Fraction

\begin{tabular}{|c|c|c|}
\hline$(1)$ & & Fraction \\
\hline & & \\
\hline Am-241 & Am-241 & $1 . \odot \odot \odot E+\odot \odot$ \\
\hline Am-241 & $\mathrm{Np}-237+\mathrm{D}$ & $1.000 \mathrm{E}+\odot \odot$ \\
\hline$n-241$ & $U-233$ & 1. $\odot \odot \odot E+\odot \odot$ \\
\hline$A m-241$ & Th-229+D & 1. $\odot \odot \odot E+\odot \odot$ \\
\hline$A m-241$ & äDSR(j) & \\
\hline Co- 60 & Co- 60 & 1. $\odot \odot \odot E+\odot \odot$ \\
\hline Cs $-137+D$ & Cs $-137+D$ & $1.000 \mathrm{E}+\odot \odot$ \\
\hline Pu-238 & Pu-238 & $1.840 \mathrm{E}-09$ \\
\hline Pu-238 & Pu-238 & 1. $\odot \odot \odot E+\odot \odot$ \\
\hline Pu-238 & U-234 & 1. $\odot \odot \odot E+\odot \odot$ \\
\hline Pu-238 & Th-230 & $1 . \odot \odot \odot E+\odot \odot$ \\
\hline $1-238$ & $\mathrm{Ra}-226+\mathrm{D}$ & $1.00 \odot \mathrm{E}+\odot \odot$ \\
\hline $\mathrm{Pu}-238$ & $P b-210+D$ & 1. $\odot \odot \odot E+\odot \odot$ \\
\hline Pu-238 & äDSR(j) & \\
\hline Pu-239 & Pu-239 & $1.000 \mathrm{E}+00$ \\
\hline $\mathrm{Pu}-239$ & $U-235+D$ & 1. $.000 \mathrm{E}+\odot \odot$ \\
\hline Pu-239 & $\mathrm{Pa}-231$ & 1. $\odot \odot \odot E+\odot \odot$ \\
\hline $1-239$ & $A C-227+D$ & $1 . \odot \odot \odot E+\odot \odot$ \\
\hline-239 & äDSR(j) & \\
\hline$-125+D$ & $S b-125+D$ & 1. $\odot \odot \odot E+\odot \odot$ \\
\hline$=$ & & $1 . \odot \odot \odot E+\odot \odot$ \\
\hline
\end{tabular}

Dose/Source Ratios Summed Over All Pathways Parent and Progeny Principal Radionuclide Contributions Indicated

DSR $(j, \mathrm{t})$ At Time in Years (mrem/yr)/(pCi/g)

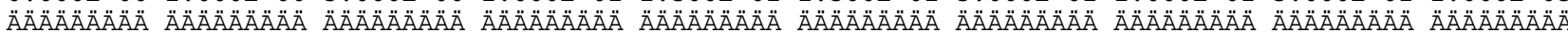

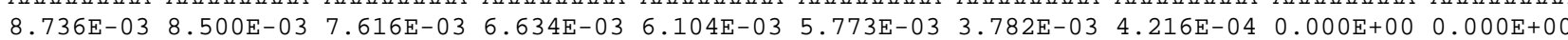

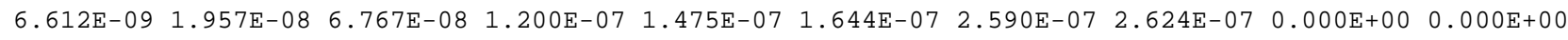
4.126E-16 2.846E-15 3.460E-14 1.155E-13 1.812E-13 $\quad 2.306 \mathrm{E}-13 \quad 6.836 \mathrm{E}-13$ 1.624E-12 $0.000 \mathrm{E}+00 \quad 0.000 \mathrm{E}+0 \odot$

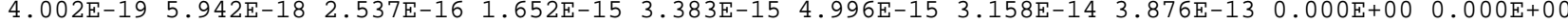
8.736E-03 8.500E-03 7.616E-03 6.634E-03 6.104E-03 $\quad 5.773 \mathrm{E}-03 \quad 3.782 \mathrm{E}-03 \quad 4.219 \mathrm{E}-04 \quad 0.000 \mathrm{E}+00 \quad 0.000 \mathrm{E}+00$ . 1.204E-11 1.187E-11 $1.118 E-111$. $1.036 E-11$ 9.898E

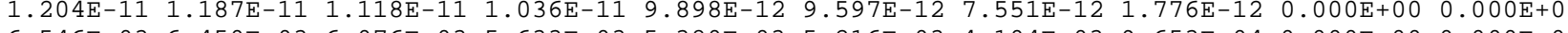
$6.546 \mathrm{E}-03 \quad 6.450 \mathrm{E}-03$ 6.076E- 03 5.633E- 03 5.380E- 03 5.216E-03 4.104E-03 9.653E-04 $0.000 \mathrm{E}+000.000 \mathrm{E}+00$

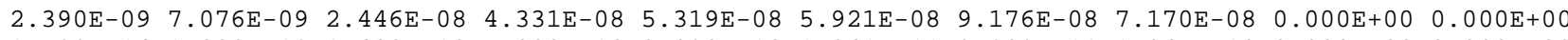

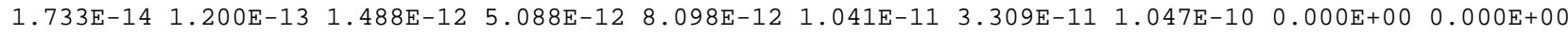
$1.298 \mathrm{E}-16$ 1.932E-15 8.336E-14 5.501E-13 $1.135 \mathrm{E}-12$ 1.685E-12 $1.104 \mathrm{E}-11$ 1.527E-10 $0.000 \mathrm{E}+00 \quad 0.000 \mathrm{E}+00$

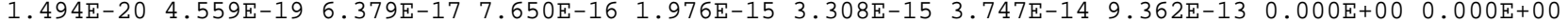
6.546E-03 6.450E-03 6.076E-03 5.633E-03 5.380E-03 5.216E-03 4.104E-03 9.654E-04 0.000E+00 $0.000 \mathrm{E}+00$

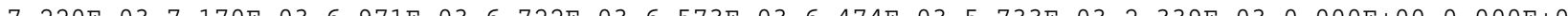

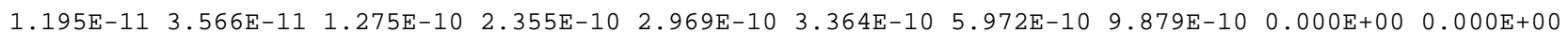

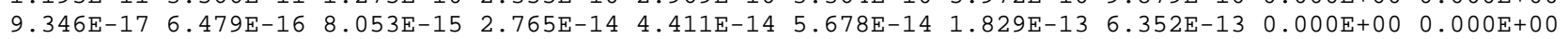

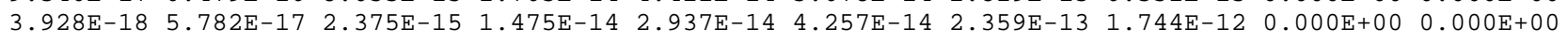

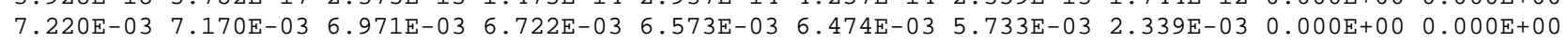
2.543E- -2 2.300E- 03 1.536E- -7 9.261E-13 6.834E-16 5.580E-18 1.213E-33 $0.000 \mathrm{E}+00 \quad 0.000 \mathrm{E}+00 \quad 0.000 \mathrm{E}+00$

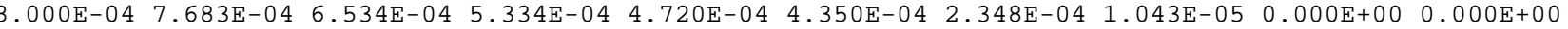

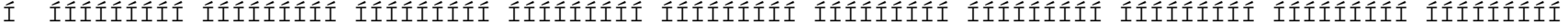

Nuclide

\begin{tabular}{|c|c|c|c|c|c|c|c|c|c|c|}
\hline $\begin{array}{c}(i) \\
\text { ÄÄÄÄÄÄÄ }\end{array}$ & 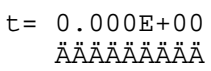 & $\begin{array}{l}1.000 E+\odot \odot \\
A \ddot{A} A \ddot{A} A ̈ A ̈ A ̈ A ̈ A ̈ A\end{array}$ & $\begin{array}{l}5.0 \odot \odot E+\odot \odot \\
A \ddot{A} A \ddot{A} A ̈ A ̈ A ̈ A ̈ A ̈ A\end{array}$ & 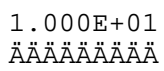 & $\begin{array}{l}1.300 E+01 \\
A \ddot{A} A \ddot{A} A ̈ A ̈ A ̈ A ̈ A ̈\end{array}$ & $\begin{array}{l}1.500 E+01 \\
\ddot{A} A \ddot{A} A \ddot{A} A ̈ A ̈ A ̈ A ̈\end{array}$ & 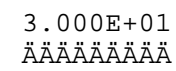 & $\begin{array}{l}1.000 E+02 \\
A \ddot{A} A \ddot{A} A ̈ A ̈ A ̈ A ̈ A ̈\end{array}$ & 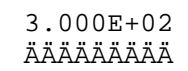 & $\begin{array}{l}1.000 E+03 \\
\ddot{A} A \ddot{A} A \ddot{A} A \ddot{A} A ̈ A ̈\end{array}$ \\
\hline Am-241 & $2.862 E+03$ & $2.941 E+03$ & $3.283 E+03$ & $3.769 \mathrm{E}+03$ & 4. $096 \mathrm{E}+03$ & $4.330 E+03$ & $6.610 E+03$ & $5.926 \mathrm{E}+04$ & $* 3.431 E+12$ & $* 3.431 \mathrm{E}+12$ \\
\hline Co- $6 \odot$ & $6.536 \mathrm{E}+01$ & $7.485 E+01$ & $1.288 E+02$ & $2.540 \mathrm{E}+\odot 2$ & $3.819 \mathrm{E}+02$ & $5.013 E+02$ & $3.882 E+03$ & $7.104 \mathrm{E}+07$ & *1.132E+15 & *1. $132 \mathrm{E}+15$ \\
\hline Cs -137 & $2.752 \mathrm{E}+\odot 2$ & $2.825 E+\odot 2$ & $3.139 E+02$ & $3.584 \mathrm{E}+02$ & $3.882 \mathrm{E}+02$ & 4. $096 \mathrm{E}+02$ & $6.150 \mathrm{E}+\odot 2$ & $5.301 \mathrm{E}+03$ & *8.704E+13 & *8.704E+13 \\
\hline Pu-238 & $3.819 E+03$ & $3.876 E+03$ & $4.115 E+03$ & $4.438 E+03$ & $4.647 \mathrm{E}+03$ & $4.793 \mathrm{E}+03$ & $6.092 \mathrm{E}+03$ & $2.590 \mathrm{E}+04$ & $* 1.712 \mathrm{E}+13$ & *1.712E+13 \\
\hline Pu-239 & $3.462 \mathrm{E}+03$ & $3.487 \mathrm{E}+03$ & $3.586 \mathrm{E}+03$ & $3.719 \mathrm{E}+03$ & $3.803 \mathrm{E}+03$ & $3.862 \mathrm{E}+03$ & $4.361 \mathrm{E}+03$ & 1. $069 \mathrm{E}+04$ & ${ }^{*} 6.214 \mathrm{E}+10$ & ${ }^{*} 6.214 \mathrm{E}+10$ \\
\hline$S b-125$ & $9.833 \mathrm{E}+02$ & $1.087 E+\odot 4$ & $1.628 \mathrm{E}+08$ & $2.700 \mathrm{E}+13$ & *1. $033 E+15$ & *1. $033 E+15$ & *1. $033 \mathrm{E}+15$ & *1. $033 \mathrm{E}+15$ & $3 E+15$ & *1. $033 \mathrm{E}+15$ \\
\hline $\mathrm{Sr}-90$ & $3.125 \mathrm{E}+04$ & $3.254 \mathrm{E}+04$ & $3.826 \mathrm{E}+04$ & $4.687 \mathrm{E}+04$ & $5.296 \mathrm{E}+04$ & $5.747 \mathrm{E}+\odot 4$ & $1.065 \mathrm{E}+05$ & $2.398 \mathrm{E}+06$ & $\star 1.365 E+14$ & $\star 1.365 \mathrm{E}+14$ \\
\hline Ííííííí & Íííííííí & Ííííííííí & Ííííííííí & Ííííííííí & Ííííííííí & Ííííííííí & Ííííííííí & Ííííííííí & ÍÍÍÍÍíííí & Ííííííííí \\
\hline
\end{tabular}

Single Radionuclide Soil Guidelines $\mathrm{G}(\mathrm{i}, \mathrm{t})$ in $\mathrm{pCi} / \mathrm{g}$ Basic Radiation Dose Limit $=2.500 \mathrm{E}+01 \mathrm{mrem} / \mathrm{yr}$ 
Summed Dose/Source Ratios DSR(i,t) in (mrem/yr)/(pCi/g)

and Single Radionuclide Soil Guidelines $G(i, t)$ in $\mathrm{pCi} / \mathrm{g}$

at $\operatorname{tmin}=$ time of minimum single radionuclide soil guideline

and at $\operatorname{tmax}=$ time of maximum total dose $=0.000 \mathrm{E}+00$ years

\begin{tabular}{|c|c|c|c|c|c|c|}
\hline $\begin{array}{l}\text { Juclide } \\
\text { (i) }\end{array}$ & $\begin{array}{l}\text { Initial } \\
(\mathrm{pCi} / \mathrm{g})\end{array}$ & $\begin{array}{c}\text { tmin } \\
(\text { years })\end{array}$ & $\operatorname{DSR}(i, \operatorname{tmin})$ & $\begin{array}{c}\mathrm{G}(\mathrm{i}, \mathrm{tmin}) \\
(\mathrm{pCi} / \mathrm{g})\end{array}$ & $\operatorname{DSR}(i, \operatorname{tmax})$ & $\begin{array}{c}\mathrm{G}(\mathrm{i}, \mathrm{tmax}) \\
(\mathrm{pC} i / \mathrm{g})\end{array}$ \\
\hline Am- & 2.8 & & & & & \\
\hline Co- 60 & & & & $6.536 \mathrm{E}+01$ & & \\
\hline & 3.8 & & & $2.752 \mathrm{E}+02$ & & \\
\hline & & & & & & \\
\hline $\mathrm{Pu}-2$ & 9.6 & & & 3.4 & & \\
\hline$S b-125$ & $3 . \odot 0 \odot E+\odot \odot$ & $\odot . \odot \odot \odot E+\odot \odot$ & & $9.833 \mathrm{E}+02$ & $E-02$ & $9.833 E+C$ \\
\hline & & & & & & \\
\hline & Íííííííí & Íííííííí́ & ÍÍÍííííí & Íí & Ííííííííí & ííí \\
\hline
\end{tabular}


onuclide Parent THF(i) (j) (i)

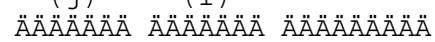
Am-241 Am-241 1.000E+0○ ONp-237 Am-241 1.000E+O० OU-233 Am-241 1. $200 \mathrm{E}+00$ OTh-229 Am-241 1.000E+०0

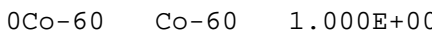
๑CS -137 Cs $-137 \quad 1.000 E+00$ OCS -137 CS -137 1.00OE+๑O OPu-238 $\mathrm{Pu}-238$ 1.840E-09 $\begin{array}{lll}\mathrm{Pu}-238 & \mathrm{Pu}-2381 & 1.000 \mathrm{E}+00\end{array}$ $\mathrm{Pu}-238$ äDOSE $(j)$

๑U-234 Pu-238 $1.000 \mathrm{E}+0 \odot$ ०Th-230 Pu-238 1.000E +00 ORa-226 Pu-238 1.000E+๑० $\odot \mathrm{Pb}-210 \quad \mathrm{Pu}-238 \quad 1.000 \mathrm{E}+\odot \odot$ बPu-239 Pu-239 $1.000 \mathrm{E}+00$ OU 235 Pu-239 $1.000 E+00$ OU -235 PU $2391.000 \mathrm{E}+0$ OPa-231 $\mathrm{Pu}-239$ 1. $000 \mathrm{E}+00$ ๑Ac-227 Pu-239 1.000E+O OSb-125 Sb-125 1.000E+०O $\odot \mathrm{Sr}-90 \quad \mathrm{Sr}-90 \quad 1.00 \Theta \mathrm{E}+\Theta \odot$ Íííííí íííííí íííííííi
Individual Nuclide Dose Summed Over All Pathways

Parent Nuclide and Branch Fraction Indicated

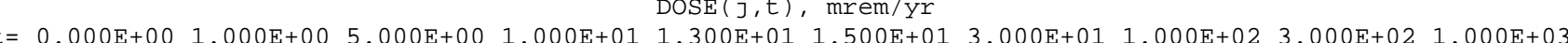

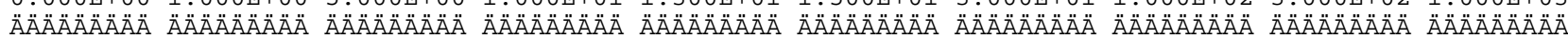

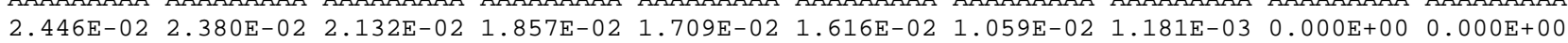

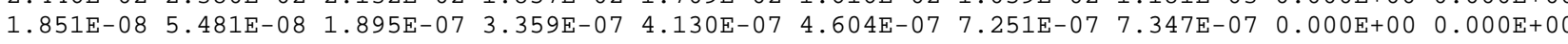
1.155E-15 7.970E-15 9.688E-14 3.234E-13 5.073E-13 $\quad 6.458 \mathrm{E}-13$ 1.914E-12 $4.548 \mathrm{E}-12 \quad 0.000 \mathrm{E}+00 \quad 0.000 \mathrm{E}+00$ $1.121 \mathrm{E}-18$ 1.664E-17 7.103E-16 4.626E-15 9.473E-15 1.399E-14 8.842E-14 1.085E-12 0.000E+00 $0.000 \mathrm{E}+00$

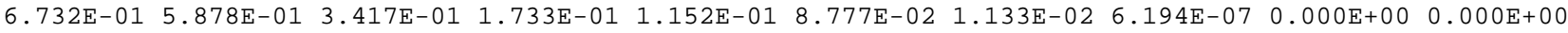
$\begin{array}{llllllllllllllll}3.470 \mathrm{E}+01 & 3.380 \mathrm{E}+01 & 3.042 \mathrm{E}+01 & 2.664 \mathrm{E}+01 & 2.460 \mathrm{E}+01 & 2.332 \mathrm{E}+01 & 1.553 \mathrm{E}+01 & 1.801 \mathrm{E}+00 & 0.000 \mathrm{E}+00 & 0.000 \mathrm{E}+00\end{array}$ $1.241 \mathrm{E}-11$ 1.222E-11 $1.152 \mathrm{E}-11$ 1. $068 \mathrm{E}-11$ 1. $220 \mathrm{E}-11 \quad 9.884 \mathrm{E}-12 \quad 7.778 \mathrm{E}-121.829 \mathrm{E}-120.000 \mathrm{E}+000.000 \mathrm{E}+00$

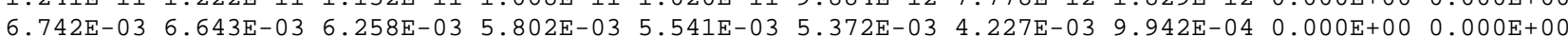

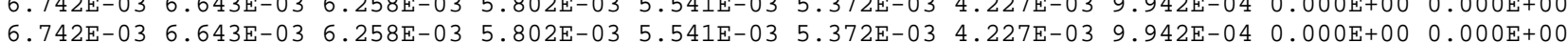

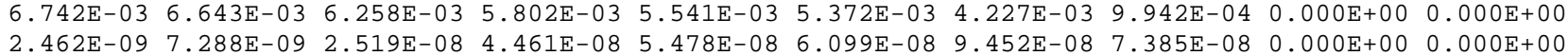

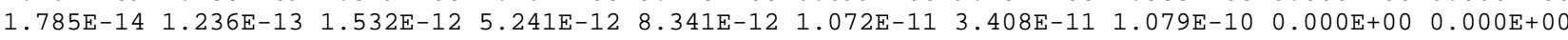
1.337E-16 1.990E-15 8.586E-14 5.666E-13 1.169E-12 $1.735 \mathrm{E}-12$ 1.137E-11 $1.573 \mathrm{E}-10 \quad 0.000 \mathrm{E}+00 \quad 0.000 \mathrm{E}+00$

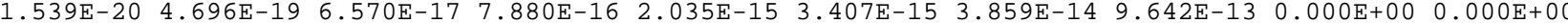
6.932E-02 6.884E-02 6.692E-02 6.453E-02 6.310E-02 6.215E-02 5.503E-02 2.245E-02 $0.000 \mathrm{E}+00 \quad 0.000 \mathrm{E}+00$

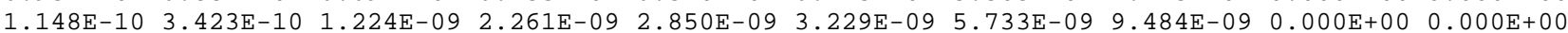
8.972E-16 $6.220 \mathrm{E}-15 \quad 7.731 \mathrm{E}-14$ 2.654E-13 $4.234 \mathrm{E}-13 \quad 5.451 \mathrm{E}-13$ 1.756E-12 $\quad 6.098 \mathrm{E}-12 \quad 0.000 \mathrm{E}+00 \quad 0.000 \mathrm{E}+00$

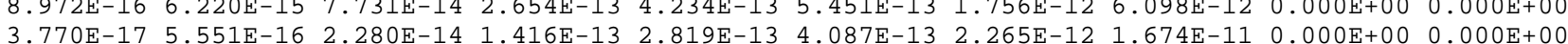

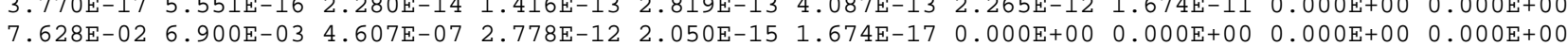

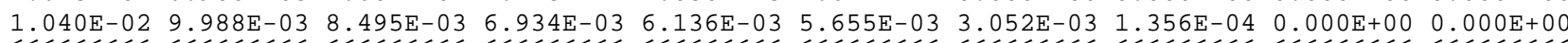

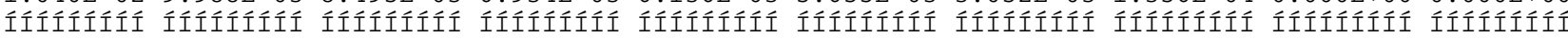


Individual Nuclide Soil Concentration

ONuclide Parent THF(i) ( j ) (i) (i) Am-241 Am 241 1 . AM-241 Am-241 1.00OE+OO Am-241 $1.00 \odot E+\odot 0$ ๑U-233 Am-241 1.000E+०० OTh-229 Am-241 1.000E+@० ०Co-60 Co-60 1.000E+00 $\Theta \mathrm{CS}-137$ CS $-137 \quad 1.000 \mathrm{E}+00$ OCS -137 CS $-137,1.000+00$ $\odot$ Pu-238 Pu-238 1.840E- 09 $\begin{array}{lll}\mathrm{Pu}-238 & \mathrm{Pu}-238 & 1.000 \mathrm{E}+00 \\ \mathrm{Pu}-238 & \text { äS }(\mathrm{j}): & \end{array}$ $\mathrm{Pu}-238$ äS $(\mathrm{j})$ :

०U-234 PU-238 $1.000 \mathrm{E}+00$ OTh-230 Pu-238 $1.000 \mathrm{E}+00$ ORa-226 Pu-238 1.000E +00 $\odot \mathrm{Pb}-210 \quad \mathrm{Pu}-238 \quad 1.000 \mathrm{E}+00$ बPu-239 Pu-239 $1.000 E+00$ OU 235 PU 239 1.000E+00 OU -235 PU $2391.000 \mathrm{E}+00$ OPA-231 $\mathrm{PU}-239$ 1. $1.000 \mathrm{E}+00$ $\odot A C-227$ PU $-239 \quad 1.000 \mathrm{E}+00$ $0 \mathrm{Sb}-125 \mathrm{Sb}-125 \quad 1.000 \mathrm{E}+00$ $\odot \mathrm{Sr}-9 \odot \quad \mathrm{Sr}-9 \odot \quad 1.0 \odot \odot \mathrm{E}+\odot \odot$

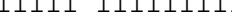
ORESCALC. EXE execution time
Parent Nuclide and Branch Fraction Indicated

$$
\mathrm{S}(j, \mathrm{t}), \mathrm{pCi} / \mathrm{g}
$$

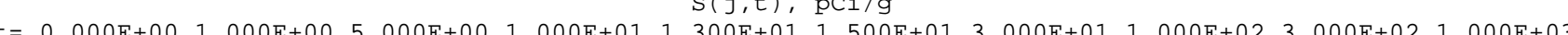

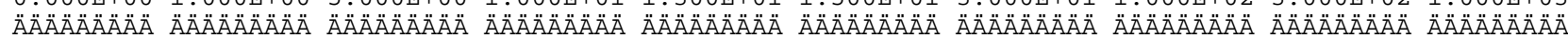

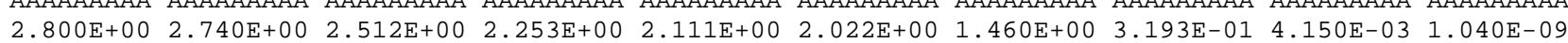
$0.000 \mathrm{E}+0 \odot$ 8.964E- 07 4.280E-06 8.086E-06 1.016E-05 1.147E-05 1.948E-05 3.333E-05 2.800E-05 9.310E-06

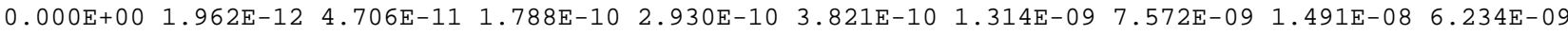
$0.000 \mathrm{E}+0 \odot \quad 6.193 \mathrm{E}-17 \quad 7.503 \mathrm{E}-15$ 5.774E-14 1.240E-13 1.875E-13 $1.339 \mathrm{E}-12 \quad 3.040 \mathrm{E}-11 \quad 2.668 \mathrm{E}-10 \quad 9.286 \mathrm{E}-10$ $\begin{array}{llllllllll}1.760 \mathrm{E}+00 & 1.543 \mathrm{E}+00 & 9.101 \mathrm{E}-01 & 4.706 \mathrm{E}-01 & 3.168 \mathrm{E}-01 & 2.433 \mathrm{E}-01 & 3.364 \mathrm{E}-02 & 3.287 \mathrm{E}-06 & 1.147 \mathrm{E}-17 & 0.000 \mathrm{E}+00\end{array}$

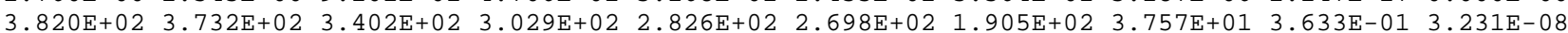
$1.895 \mathrm{E}-09$ 1.880E-09 $1.820 \mathrm{E}-09$ 1.748E-09 $1.706 \mathrm{E}-09$ 1.678E-09 $1.486 \mathrm{E}-09$ 8.428E-10 $1.667 \mathrm{E}-105.735 \mathrm{E}-13$

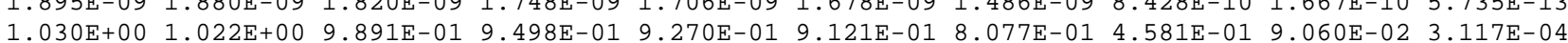

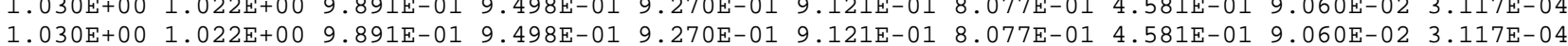

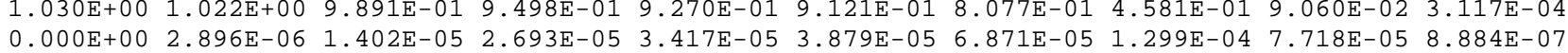
$\odot .000 \mathrm{E}+0 \odot$ 1.307E-11 3.198E-10 1.245E-09 2.071E-09 2.728E-09 1.007E-08 7.801E-08 2.791E-07 $3.950 \mathrm{E}-07$ $0.000 \mathrm{E}+0 \odot$ 1.887E-15 2.307E-13 1.795E-12 3.877E-12 5.890E-12 4.335E-11 $1.096 \mathrm{E}-09$ 1.063E-08 2.701E-08

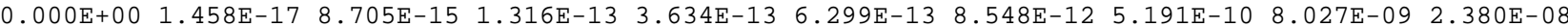

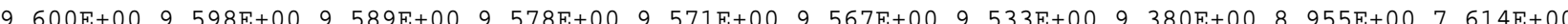
. (1)

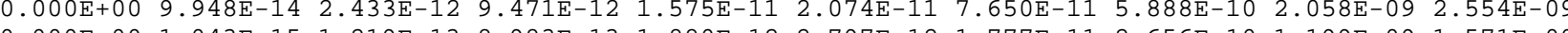

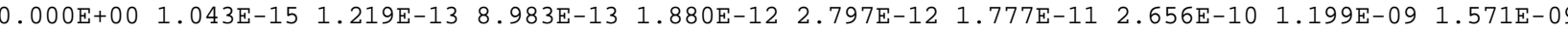

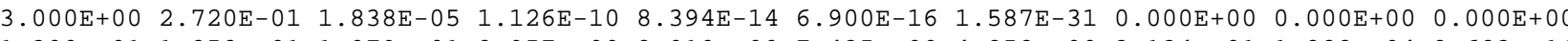

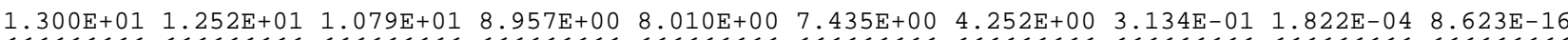

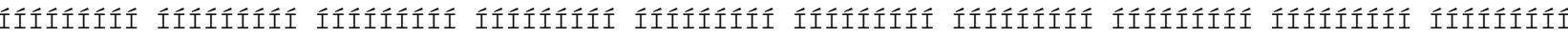
the parent nuclide.

4.23 seconds 


\section{Appendix E}

Closure Summary

Uncontrolled When Printed 
A land use restriction will be applied as part of the closure in place alternative for CAU 476. This use restriction will also cover CAU 559 as stated in the CAU 559 $\mathrm{CADD} / \mathrm{CR}$. The use restrictions will be applied to control use and limit access to the sites to prevent inadvertent exposure to the radionuclide contaminated soil identified in the muckpile (CAS 12-06-02) and the TPH-DRO contaminated soil found on the Compressor/Blower Pad (CAS 12-25-13). The completed land use restriction form and map are included in this appendix.

The following warning sign will appear on or adjacent to the T-Tunnel gate which controls access to CAU 476, CAU 478, CAU 559, and CAU 309:

\section{WARNING}

Radiologically Contaminated Areas Beyond This Point

FFACO Sites

CAU 476, Area 12, T-Tunnel Sites CAS 12-06-02, Muckpile

CAU 559, T-Tunnel Compressor/Blower Pad CAS 12-25-13, Oil Stained Soil and Concrete

CAU 478, Area 12, T-Tunnel Ponds CAS 12-23-01, Ponds [5], RAD Area

CAU 309, Area 12, Muckpiles CAS 12-06-09, Muckpile CAS 12-08-02, Contaminated Waste Dumps CAS 12-28-01, I, J, and K-Tunnel Debris

Access to this area is not permitted without U.S. Government permission

Before working in this area, Contact Real Estate Services at 295-2528

This site can be closed without further corrective action. 


\section{CAU Use Restriction Information}

CAU Number/Description: 476/Area 12 T-Tunnel Sites, 559/T-Tunnel Compressor/Blower Pad

Applicable CAS Numbers/Descriptions: 12-06-02/Muckpile, 12-25-13/Oil Stained Soil and Concrete

Contact (organization/project: DTRA/Environmental Restoration

Surveyed Area (UTM, Zone 11, NAD 27, meters): See Attached Figure

NE Corner $\quad 4,119,052.4 \mathrm{~N} \quad 574,276.4 \mathrm{E}$

N Center $\quad 4,119,051.6 \mathrm{~N} \quad 574,063.1 \mathrm{E}$

NW Comer $4,118,959.4 \mathrm{~N} 573,850.1 \mathrm{E}$

SW Corner $4,118,837.6 \mathrm{~N} 573,850.5 \mathrm{E}$

SE Corner $4,118,839.1 \mathrm{~N} 574,277.1 \mathrm{E}$

Survey Date: $\underline{7 / 10 / 2005} \quad$ Survey Method (GPS, etc): $\underline{\text { GPS }}$

Site Monitoring Requirements: Certify that posting is in place, in tact, and readable

Required Frequency (quarterly, annually?): Annually

If Monitoring Has Started, Indicate last Completion Date:

\section{Use Restrictions}

The future use of any land related to this Corrective Action Unit (CAU), as described by the above surveyed location, is restricted from any DOE or Air Force activity that may alter or modify the containment control as approved by the state and identified in the CAU Closure Report or other CAU documentation unless appropriate concurrence is obtained in advance.

Comments: See the Closure Report for additional information on the condition of the site(s). Results of the annual inspection will be provided in the annual Post Closure Inspection Monitoring Report.

Submitted By:

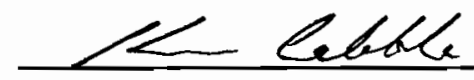
Date:

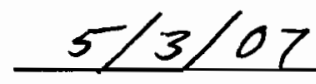

cc with copy of survey map (paper and digital (dgn) formats):

CAU Files ( 2 copies) 


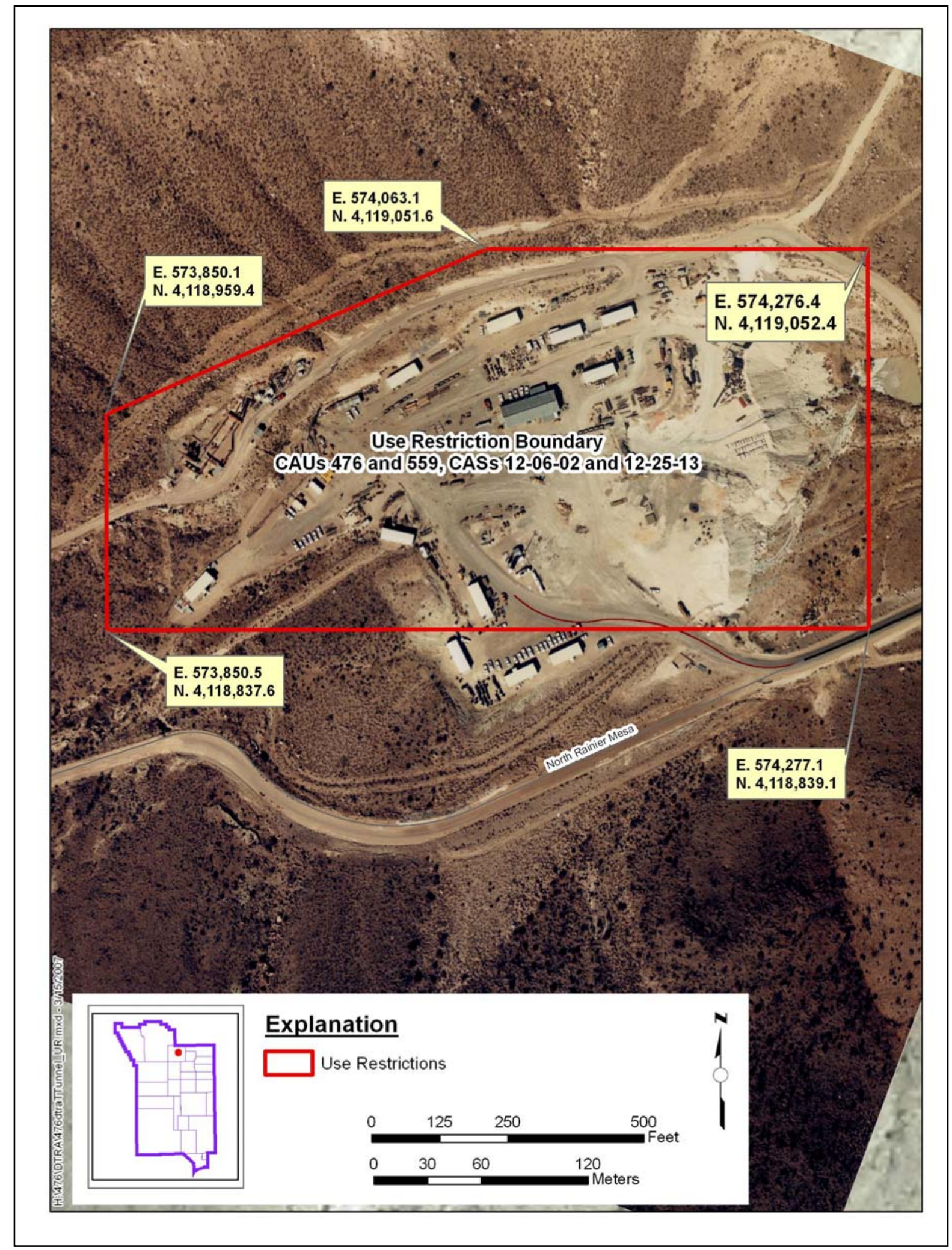

Figure E.1-1

CAU 476 Area 12 T-Tunnel Sites 


\section{LIBRARY DISTRIBUTION LIST}

U.S. Department of Energy

National Nuclear Security Administration

Nevada Site Office

Technical Library

P.O. Box 98518, M/S 505

Las Vegas, NV 89193-8518

U.S. Department of Energy

Office of Scientific and Technical Information

P.O. Box 62

Oak Ridge, TN 37831-0062

Southern Nevada Public Reading Facility

c/o Nuclear Testing Archive

P.O. Box 98521, M/S 400

Las Vegas, NV 89193-8521

Manager, Northern Nevada FFACO

Public Reading Facility

c/o Nevada State Library \& Archives

Carson City, NV 89701-4285
1 (Uncontrolled, electronic copy)

1 (Uncontrolled, electronic copy)

2 (Uncontrolled, electronic copies)

1 (Uncontrolled, electronic copy) 